\title{
Pentaquark and Tetraquark states
}

Yan-Rui Liu, ${ }^{1 *}$ Hua-Xing Chen, ${ }^{2 *}$ Wei Chen, ${ }^{3 *}$ Xiang Liu, ${ }^{4,5 \dagger}$ Shi-Lin Zhu ${ }^{6,7,8 \ddagger}$

${ }^{1}$ School of Physics, Shandong University, Jinan 250100, China

${ }^{2}$ School of Physics, Beihang University, Beijing 100191, China

${ }^{3}$ School of Physics, Sun Yat-Sen University, Guangzhou 510275, China

${ }^{4}$ School of Physical Science and Technology,

Lanzhou University, Lanzhou 730000, China

${ }^{5}$ Research Center for Hadron and CSR Physics, Lanzhou University and

Institute of Modern Physics of CAS, Lanzhou 730000, China

${ }^{6}$ School of Physics and State Key Laboratory of Nuclear Physics and Technology, Peking University, Beijing 100871, China

${ }^{7}$ Collaborative Innovation Center of Quantum Matter, Beijing 100871, China

${ }^{8}$ Center of High Energy Physics, Peking University, Beijing 100871, China

April 2, 2019

\begin{abstract}
The past seventeen years have witnessed tremendous progress on the experimental and theoretical explorations of the multiquark states. The hidden-charm and hidden-bottom multiquark systems were reviewed extensively in Ref. [1]. In this article, we shall update the experimental and theoretical efforts on the hidden heavy flavor multiquark systems in the past three years. Especially the LHCb collaboration not only confirmed the existence of the hidden-charm pentaquarks but also provided strong evidence of the molecular picture. Besides the well-known $X Y Z$ and $P_{c}$ states, we shall discuss more interesting tetraquark and pentaquark systems either with one, two, three or even four heavy quarks. Some very intriguing states include the fully heavy exotic tetraquark states $Q Q \bar{Q} \bar{Q}$ and doubly heavy tetraquark states $Q Q \bar{q} \bar{q}$, where $Q$ is a heavy quark. The $Q Q \bar{Q} \bar{Q}$ states may be produced at LHC while the $Q Q \bar{q} \bar{q}$ system may be searched for at BelleII and $\mathrm{LHCb}$. Moreover, we shall pay special attention to various theoretical schemes such as the chromomagnetic interaction (CMI), constituent quark model, meson exchange model, heavy quark and heavy diquark symmetry, QCD sum rules, Faddeev equation for the three body systems, Skyrme model and the chiral quark-soliton model, and the lattice QCD simulations. We shall emphasize the model-independent predictions of various models which are truly/closely related to Quantum Chromodynamics (QCD). For example, the chromomagnetic interaction arises from the gluon exchange which is fundamental and universal in QCD and responsible for the mixing and mass splitting of the conventional mesons and baryons within the same multiplet. The same CMI
\end{abstract}

*These authors equally contribute to this work.

${ }^{\dagger}$ Corresponding author: xiangliu@lzu.edu.cn

${ }^{\ddagger}$ Corresponding author: zhusl@pku.edu.cn 
mechanism shall also be responsible for the mixing of the different color configurations and mass splittings within the multiplets in the multiquark sector. There have also accumulated many lattice QCD simulations through multiple channel scattering on the lattice in recent years, which provide deep insights into the underlying structure/dynamics of the $X Y Z$ states. In terms of the recent $P_{c}$ states, the lattice simulations of the charmed baryon and anti-charmed meson scattering are badly needed. We shall also discuss some important states which may be searched for at BESIII, BelleII and $\mathrm{LHCb}$ in the coming years.

\section{Contents}

1 Introduction $\quad 3$

$2 \quad S U(6)$ symmetry and Chromomagnetic interaction (CMI) 9

2.1 Hamiltonian for the CMI model . . . . . . . . . . . . . . . . . . . . 10

2.2 CMI models . . . . . . . . . . . . . . . . . . . . . . . . 12

2.3 CMI and Conventional hadrons . . . . . . . . . . . . . . . . . . . . . . 14

2.4 CMI and $Q \bar{Q} q \bar{q} \ldots \ldots \ldots \ldots \ldots \ldots \ldots \ldots \ldots \ldots \ldots$

2.5 CMI and $Q Q \bar{q} \bar{q} \ldots \ldots \ldots \ldots \ldots \ldots \ldots \ldots \ldots$

2.6 CMI and $Q Q \bar{Q} \bar{Q} \ldots \ldots \ldots \ldots \ldots \ldots \ldots \ldots \ldots \ldots \ldots$

$2.7 \quad \mathrm{CMI}$ and $Q Q \bar{Q} \bar{q} \ldots \ldots \ldots \ldots \ldots \ldots \ldots \ldots \ldots \ldots$

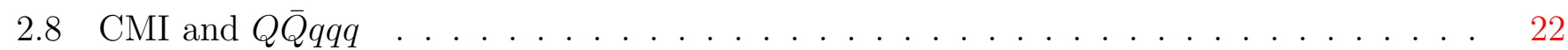

2.9 CMI and $Q Q q q \bar{q} \ldots \ldots \ldots \ldots \ldots \ldots \ldots \ldots \ldots \ldots$

$2.10 \mathrm{CMI}$ and $Q Q Q q \bar{q} \ldots \ldots \ldots \ldots \ldots \ldots \ldots \ldots \ldots$

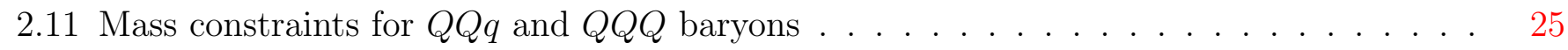

2.12 Effective CMI for multiquark states . . . . . . . . . . . . . . . . . . . . 26

2.13 A short summary for CMI . . . . . . . . . . . . . . . . . . . . 28

3 Constituent quark models $\quad 29$

$3.1 Q \bar{Q} q \bar{q} \ldots \ldots \ldots \ldots \ldots \ldots \ldots \ldots \ldots \ldots$

3.1.1 Possible quarkonium or hybrid assignments . . . . . . . . . . . . . . . 30

3.1.2 Schemes of the $Q \bar{Q}$ core admixed with coupled channels . . . . . . . . . . . 31

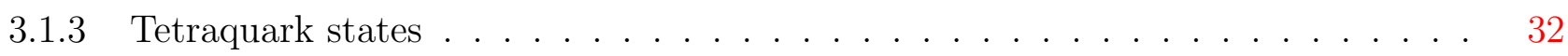

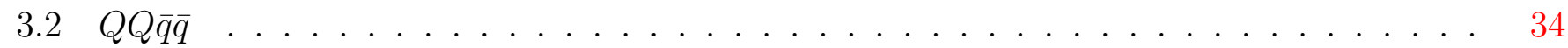

$3.3 Q Q \bar{Q} \bar{Q}$ and $Q Q \bar{Q} \bar{q} \ldots \ldots \ldots \ldots \ldots \ldots \ldots \ldots \ldots$

$3.4 Q \bar{Q} q q q$ and $Q Q q q \bar{q} \ldots \ldots \ldots \ldots \ldots \ldots \ldots \ldots$

3.5 Color flux-tube model . . . . . . . . . . . . . . . . . . . . . . 36

3.6 A short summary . . . . . . . . . . . . . . . . . . . . . . 38

4 Meson exchange and scattering methods $\quad 38$

$4.1 \quad Q \bar{Q} q \bar{q} \ldots \ldots \ldots \ldots \ldots \ldots \ldots \ldots \ldots \ldots$

$4.2 Q Q \bar{q} \bar{q} \ldots \ldots \ldots \ldots \ldots \ldots \ldots \ldots \ldots \ldots \ldots \ldots \ldots \ldots$

$4.3 Q \bar{Q} q q q \ldots \ldots \ldots \ldots \ldots \ldots \ldots \ldots \ldots \ldots \ldots$

$4.4 Q Q q q \bar{q} \ldots \ldots \ldots \ldots \ldots \ldots \ldots \ldots \ldots \ldots$

$4.5 Q Q Q q \bar{q} \ldots \ldots \ldots \ldots \ldots \ldots \ldots \ldots \ldots \ldots \ldots \ldots$

4.6 A short summary . . . . . . . . . . . . . . . . . . . . . 45 
$5 \quad$ Heavy quark symmetry and multiquark states

$5.1 Q \bar{Q} q \bar{q} \ldots \ldots \ldots \ldots \ldots \ldots \ldots \ldots$

$5.2 Q Q \bar{q} \bar{q} \ldots \ldots \ldots \ldots \ldots \ldots \ldots \ldots \ldots \ldots$

$5.3 Q \bar{Q} q q q$ and $Q Q Q q \bar{q} \ldots \ldots \ldots \ldots \ldots \ldots \ldots \ldots$

6 QCD sum rules $\quad 48$

6.1 A short induction to QCD sum rules . . . . . . . . . . . . . . . . . . . . . . . . . . . . . . .

6.2 Interpolating currents and their relations to physical states . . . . . . . . . . . . . . . . 49

$6.3 Q \bar{Q} q \bar{q}$ and $Q Q \bar{Q} \bar{Q} \ldots \ldots \ldots \ldots \ldots \ldots \ldots \ldots \ldots$

$6.4 Q Q \bar{q} \bar{q}$ and $Q Q \bar{Q} \bar{q} \ldots \ldots \ldots \ldots \ldots \ldots \ldots \ldots \ldots \ldots$

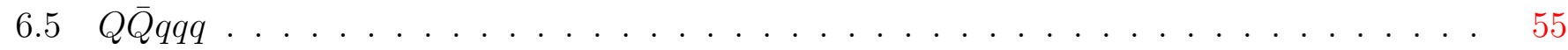

7 Three-body system $\quad 56$

7.1 A short introduction to Faddeev equations . . . . . . . . . . . . . . . . . . 56

7.2 Applications of Faddeev equations to exotic hadrons . . . . . . . . . . . . . . . 58

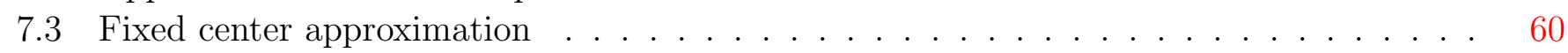

8 The Skyrme model and the chiral quark-soliton model 62

8.1 A short introduction to the Skyrme model . . . . . . . . . . . . . . . . . . 63

8.2 A short introduction to the chiral quark-soliton model . . . . . . . . . . . . . 66

9 Progresses from Lattice QCD $\quad 68$

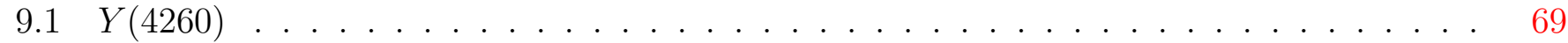

$9.2 X(3872) \ldots \ldots \ldots \ldots \ldots \ldots \ldots \ldots \ldots \ldots$

9.3 The charged $Z_{c}$ states . . . . . . . . . . . . . . . . . . . . . . . . 73

9.4 The doubly-charmed/bottom tetraquark states . . . . . . . . . . . . . . . . . 77

9.5 Other tetraquark configurations . . . . . . . . . . . . . . . . . 80

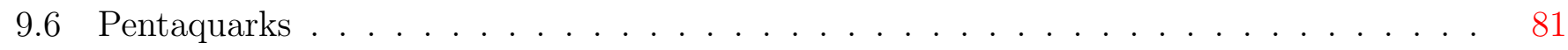

9.7 A short summary for lattice QCD . . . . . . . . . . . . . . . . . . 84

10 Production and decay properties $\quad 85$

$10.1 Q \bar{Q} q \bar{q} \ldots \ldots \ldots \ldots \ldots \ldots \ldots \ldots$

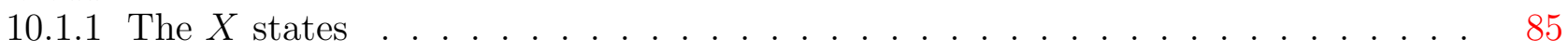

10.1 .2 The $Y$ states . . . . . . . . . . . . . . . . . . . . . . . . 88

10.1 .3 The $Z_{c}$ states . . . . . . . . . . . . . . . . . . . . . . . . . . . 89

10.1 .4 The $Z_{b}$ states . . . . . . . . . . . . . . . . . . . . . . . . . . . . 91

$10.2 Q Q \bar{q} \bar{q}$ and $Q Q \bar{Q} \bar{Q} \ldots \ldots \ldots \ldots \ldots \ldots \ldots \ldots$

$10.3 Q \bar{Q} q q q \ldots \ldots \ldots \ldots \ldots \ldots \ldots \ldots$

10.4 A short summary . . . . . . . . . . . . . . . . . . . . . . . . . . . . . . . . . . . . . .

11 Summary and perspective $\quad 95$

\section{Introduction}

In the past decades, hadron physicists show great interest in hunting for evidences of the multiquark states. As a new form of matter beyond conventional mesons $(\bar{q} q)$ and baryons $(q q q)$, multiquark states containing more than three quarks are of special importance in the hadron family. Especially, with the observations of various charmonium-like $X Y Z$ states, the study of multiquark states has entered upon a new era. 
In this article, we will give a concise review on the research progress of the tetraquark $(\bar{q} \bar{q} q q)$ and pentaquark $(\bar{q} q q q q)$ states, which are typical multiquark matters. Before doing that, let us first look back on the history of particle/hadron physics. It is well known that the development of quantum mechanics is closely related to the study of atomic spectroscopy, which reveals the mysterious veil of the atom's microstructure. Here, we find a similar situation that the study of hadron spectroscopy is bringing us new insights into the internal structure and dynamics of hadrons.

In the early 1960s, many strongly interacting particles were observed in particle/nucleon experiments, which were named as "hadron" by L. B. Okun later [2]. Based on these observations, M. GellMann and G. Zweig independently developed the classification scheme for hadrons - the quark model [3, 4, 5]. Especially, the name "quark" was given by M. Gell-Mann to denote the substructure of hadrons. The quark model achieves a great success, and it is a milestone in the development of particle physics. A well-known example is the prediction of the baryon with three strange quarks, that is the $\Omega$. It was discovered in 1964 [6] after its existence, mass, and decay products had been predicted in 1961 independently by M. Gell-Mann [7] and Y. Ne'eman [8].

Then we would like to mention the establishment of the Cornell model. In 1974, as the first state in the charmonium family, the $J / \psi$ was discovered by the E598 Collaboration [9] and the SLAC-SP017 Collaboration [10], which confirmed the existence of the charm quark [11]. After that, a series of charmonia were discovered, such as the $\psi(3686)[12], \psi(3770)[13], \psi(4040)[14], \psi(4160)[15]$, and $\psi(4415)$ [16], etc. Based on these experimental observations, the Cornell model was proposed [17, 18], which uses the Cornell potential $V(r)=-k / r+r / a^{2}[19]$ to depict the interaction between the $c h a r m$ and anti-charm quarks. This potential consists of both the Coulomb-type and linear potentials, which can well reproduce masses of the above observed charmonia at that time. Inspired by the Cornell model, various potential models were developed [20, 21, 22, 23, 24, 25, 26, 27, 28, 29, 30, 31, 32, 33], among which the Godfrey-Isgur model [33] is quite popular. This model contains the semi-relativistic kinetic and potential energy terms, which can be applied to quantitatively describe not only meson spectra [33] but also baryon spectra [34]. These phenomenological models, inspired by the charmonium family, greatly improve our understanding of the internal structure and dynamics of conventional mesons and baryons.

Nowadays several hundreds of hadrons have been observed in particle experiments, most of which can be categorized into two families: baryons made of three quarks and mesons made of one quark and one antiquark [35]. They are formed by the up, down, strange, charm, and bottom quarks/antiquarks (the top quark has a very short lifetime and therefore does not form hadrons), which are governed by the strong interaction. Although the mechanism of the color confinement remains one of the most difficult problems in particle physics, various phenomenological models were proposed to quantitatively describe the hadron spectroscopy. Due to the joint efforts from the particle/hadron theorists and experimentalists, the meson and baryon families have been nearly complete, and almost all the groundstate hadrons have been discovered. Especially, the doubly charmed baryon $\Xi_{c c}^{++}(3621)$ was recently discovered by LHCb Collaboration [36].

Other than hundreds of conventional mesons and baryons, there were only tens of multiquark candidates observed in particle experiments, although the concept of the multiquark states appeared together with the birth of the quark model. For example, M. Gell-Mann indicated [3]: "Baryons can now be constructed from quarks by using the combinations $(q q q),(q q q q \bar{q})$, etc., while mesons are made out of $(q \bar{q}),(q q \bar{q} \bar{q})$, etc", and G. Zweig [5] also wrote: "In general, we would expect that baryons are built not only from the product of these aces, $A A A$, but also from $\bar{A} A A A A, \bar{A} \bar{A} A A A A A$, etc., where $\bar{A}$ denotes an anti-ace. Similarly, mesons could be formed from $\bar{A} A, \bar{A} \bar{A} A A$, etc". Due to the constraint of reliable experimental and theoretical techniques, the study of the multiquark states was far from satisfactory before 2003. At that time, theorists mainly focused on a) the light scalar mesons $\sigma, \kappa, a_{0}(980)$, and $\left.f_{0}(980), \mathrm{b}\right)$ the scalar mesons $f_{0}(1370), f_{0}(1500)$, and $f_{0}(1710)$, and c) the $\Lambda(1405)$, and discussed their possible interpretations as multiquark states and glueballs (see review articles [37, 38] for details). 


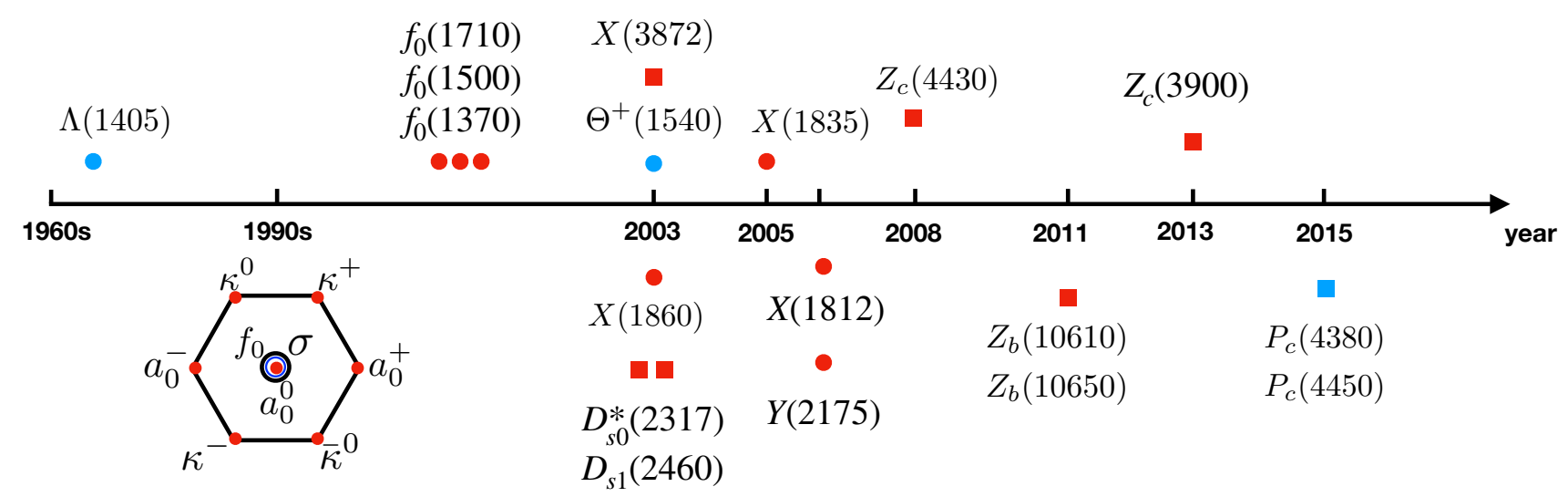

Figure 1: Observations of some typical exotic hadronic states.

2003 is an important year in the history of the multiquark states. Since 2003, there has been continuous progress in this field. With the accumulation of experimental data, a series of charmoniumlike $X Y Z$ states were reported (see Fig. 1), which stimulated us to reveal their exotic inner structures. We shall try our best to convey the progress and excitement to the readers in the present review. Here are several typical examples:

- The BES and BESIII Collaborations reported a series of light-flavor multiquark candidates, including the $X(1860)$ observed in the $J / \psi \rightarrow \gamma p \bar{p}$ decay [39], the $X(1835)$ in $J / \psi \rightarrow \gamma \eta^{\prime} \pi^{+} \pi^{-}$ $[40,41]$, the $X(1812)$ in $J / \psi \rightarrow \gamma \omega \phi[42]$, the $Y(2175)$ in $J / \psi \rightarrow \eta \phi f_{0}(980)[43,44]$, and so on. These observations inspired extensive discussions, and various exotic interpretations such as multiquark states were proposed. Since the present review mainly focuses on the heavy-flavor multiquark states, interested readers may consult Ref. [38] for more information.

- In 1997, Diakonov et al. predicted the existence of a light-flavor pentaquark with the chiral soliton model [45], which has a mass around $1530 \mathrm{MeV}$, spin 1/2, isospin 0 and strangeness +1 . In 2003, LEPS Collaboration claimed that a narrow $\Theta^{+}(1540)$ particle consistent with the above prediction was observed in the $\gamma n \rightarrow K^{+} K^{-} n$ reaction [46]. Very quickly, the $\Theta^{+}(1540)$, as a pentaquark candidate, became a super star at that time [47]. Unfortunately, it was not confirmed by the subsequent series of high precise experiments [48]. The $\Theta^{+}(1540)$ is probably not a genuine resonance [49]. The rise and fall of the $\Theta^{+}(1540)$ unveiled our ignorance on the non-perturbative behavior of quantum chromodynamics. The lessons and experience with the $\Theta^{+}(1540)$ have been helpful in the search of the pentaquark and tetraquark states.

- In 2003, BaBar Collaboration observed a narrow heavy-light state $D_{s 0}^{*}(2317)$ in the $D_{s}^{+} \pi^{0}$ invariant mass spectrum [50]. Since the mass of the $D_{s 0}^{*}(2317)$ is about $100 \mathrm{MeV}$ lower than the quark model prediction of the $P$-wave charmed-strange meson with $J^{P}=0^{+}$[33], its tetraquark explanation was proposed in Refs. [51, 52, 53]. A similar situation happened to the $D_{s 1}(2460)$ observed by CLEO Collaboration [54]. The strong channel coupling between the S-wave $\operatorname{DK}\left(K^{*}\right)$ scattering states and bare quark model $c \bar{s}$ states plays a pivotal role in lowering the masses of the $D_{s 0}^{*}(2317)$ and $D_{s 1}(2460)[55,56]$.

- Still in 2003, $X(3872)$, as the first particle in charmonium-like $X Y Z$ family, was discovered by Belle Collaboration [57]. Since the mass of the $X(3872)$ is close to the threshold of the $D \bar{D}^{*}$ pair, its assignment as the $D \bar{D}^{*}$ molecular state is very popular. We note that its nature is still under heated debates today. Similar to the $D_{s 0}^{*}(2317)$ and $D_{s 1}(2460)$, there also exists a low mass 
puzzle related to the $X(3872)$. Its mass is much lower than the quark model prediction of the charmonium $\chi_{c 1}(2 P)$ state [33]. Again, the coupled channel effect may mediate this difference [58].

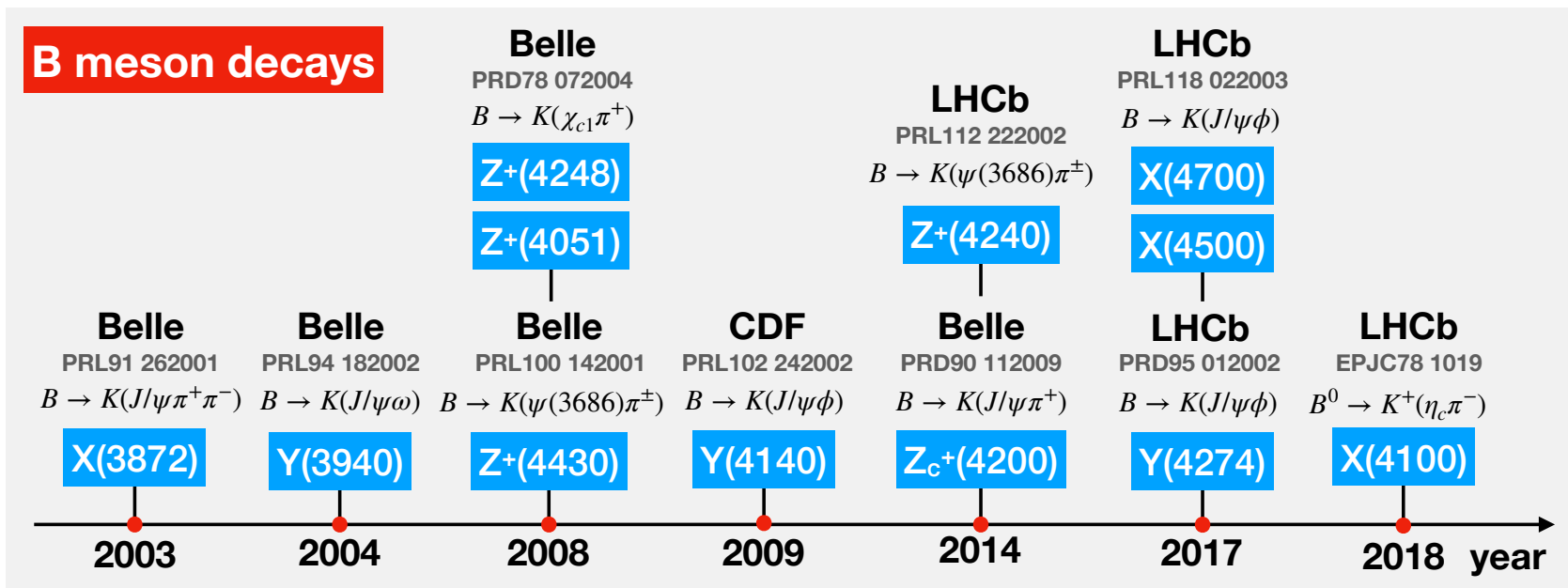

\section{The $e^{+} e^{-}$annihilation}

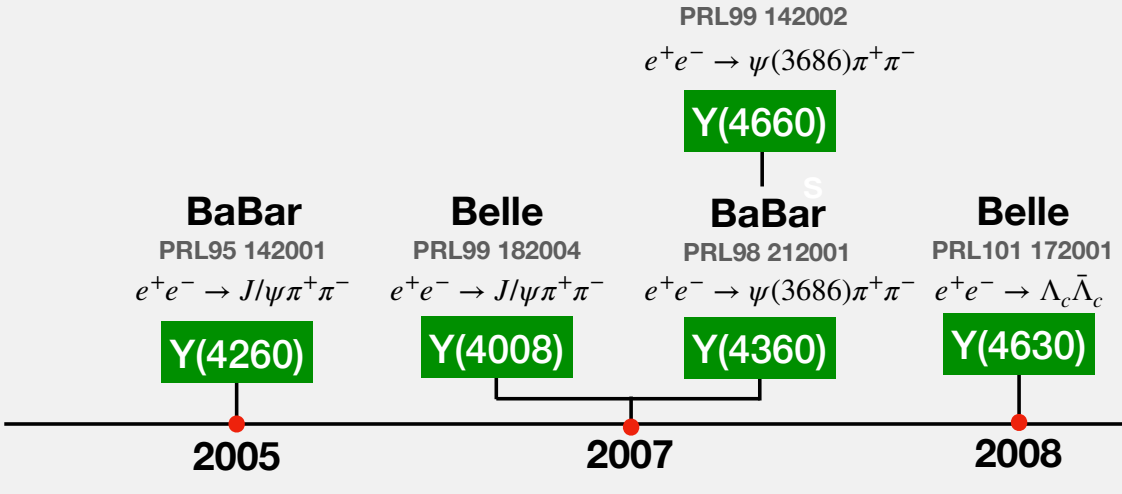

BESIII

PRL118 092002

$e^{+} e^{-} \rightarrow h_{c} \pi^{+} \pi^{-}$

$\mathrm{X}(4390)$

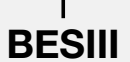

PRL118 092001

$e^{+} e^{-} \rightarrow J / \psi \pi^{+} \pi^{-}$

$\mathrm{X}(4320)$

$\mathrm{Y}(4220)$ $2017 \quad$ year

Figure 2: Charmonium-like $X Y Z$ states from $B$ meson decays and the $e^{+} e^{-}$annihilation.

In the past 17 years, more and more candidates of the exotic hadrons were observed in the Belle, BaBar, BESIII, D0, CDF, CMS, and LHCb experiments, such as a) the charmonium-like $X Y Z$ states $Y(4260), Z_{c}(4430)$, and $Z_{c}(3900)$, b) the bottomonium-like states $Z_{b}(10610)$ and $Z_{b}(10650)$, and c) the hidden-charm pentaquark states $P_{c}(4380)$ and $P_{c}(4450)$, etc. In Figs. 2-3, we summarize these observations concisely. An extensive review of the states observed before 2016 can be found in Ref. [1]. Here we briefly introduce the experimental observations after 2016:

- In the $J / \psi \phi$ invariant mass spectrum of the $B \rightarrow K J / \psi \phi$ decay, LHCb established the existence of the $Y(4274)$ structure with $6 \sigma$ significance $[59,60]$. This state has a mass $M=4273.3 \pm 8.3_{-3.6}^{+17.2}$ $\mathrm{MeV}$, width $\Gamma=56.2 \pm 10.9_{-11.1}^{+8.4} \mathrm{MeV}$, and quantum numbers $J^{P C}=1^{++}$. Before this observation, $\mathrm{CDF}$ and CMS had reported an evidence of a structure around $4274 \mathrm{MeV}$ in the $J / \psi \phi$ invariant mass spectrum [61, 62]. Besides the $Y(4274)$ structure, two extra structures with higher masses were found in the $J / \psi \phi$ invariant mass spectrum, which are the $X(4500)$ and $X(4700)$ with 


\section{The double charmonium production process}

\begin{tabular}{cccc} 
Belle & Belle & Belle \\
PRL98 082001 & PRL100 202001 & \\
$e^{+} e^{-} \rightarrow J / \psi\left(\bar{D} D^{*}\right)$ & $e^{+} e^{-} \rightarrow J / \psi\left(D^{*+} D^{*-}\right)$ & PRD95 112003 & \\
$\mathrm{X}(3940)$ & $\mathrm{Y}(4160)$ & $e^{+} e^{-} \rightarrow J / \psi(D \bar{D})$ & \\
\hline 2007 & & $\mathrm{X}^{*}(3860)$ & year
\end{tabular}

\section{The yy fusion process}

Belle

PRL96 082003

$\gamma \gamma \rightarrow(D \bar{D})$

Z(3930)
Belle

PRL104 092001

$\gamma \gamma \rightarrow(J / \psi \omega)$

X(3915)

\section{Belle}

PRL104 112004

$\gamma \gamma \rightarrow(J / \psi \phi)$

$\mathrm{X}(4350)$

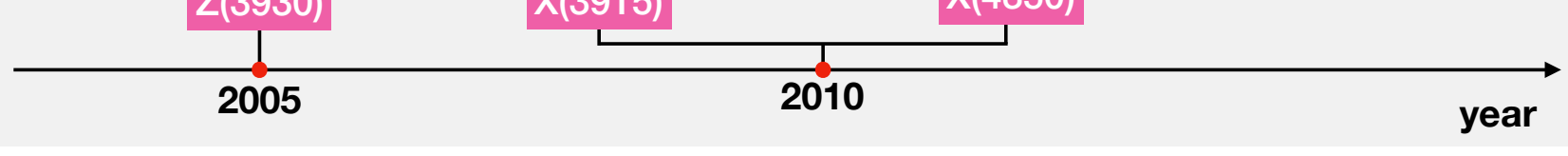

\section{The hadronic decays of $Y(4260)$}

BESIII

PRL112 132001

$e^{+} e^{-} \rightarrow Y(4260) \rightarrow\left(D^{*} \bar{D}^{*}\right)^{+} \pi^{-}$

$\mathrm{Z}_{\mathrm{c}}(4025)$

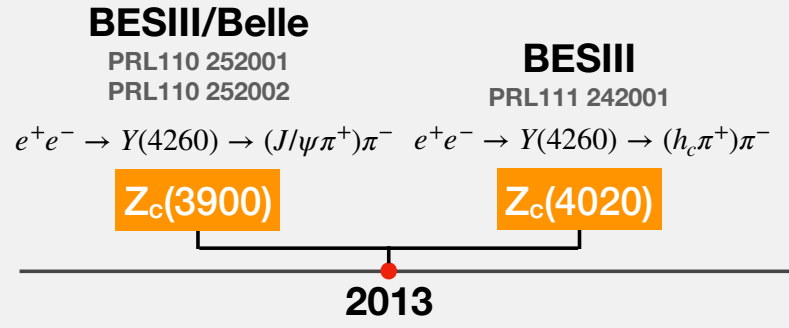

\section{BESIII}

BESIII

PRL112 022001

BESIII

PRD96 032004

$e^{+} e^{-} \rightarrow Y(4260) \rightarrow\left(D \bar{D}^{*}\right)^{+} \pi^{-} \quad e^{+} e^{-} \rightarrow Y(4260) \rightarrow\left(\psi(3686) \pi^{+}\right) \pi^{-}$

\section{$Z_{c}(3885)$}

2014

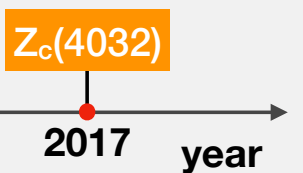

\section{Other processes}

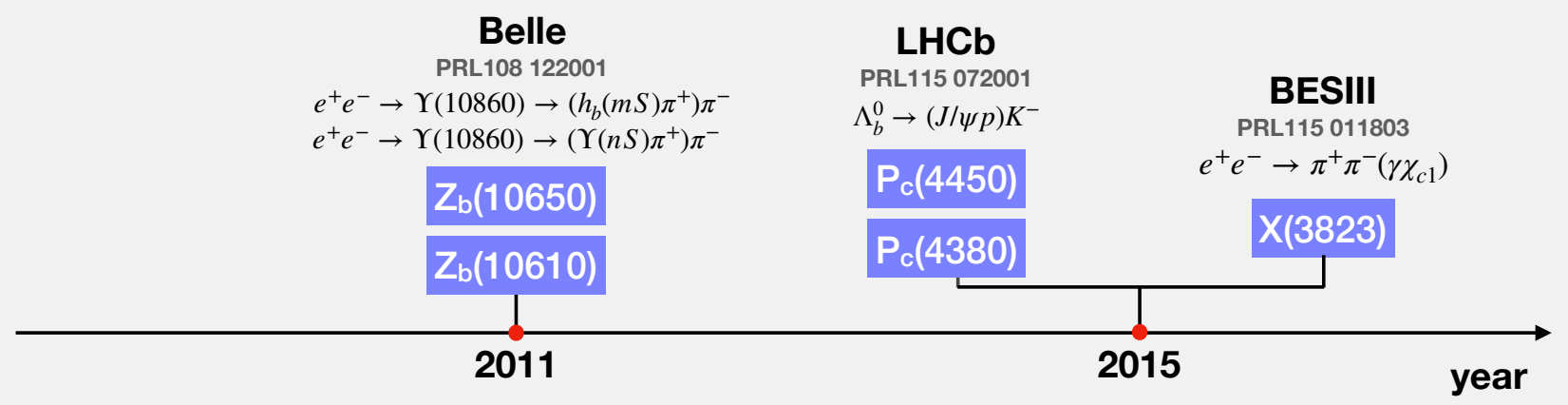

Figure 3: Charmonium-like $X Y Z$ states from the double charmonium production process, the $\gamma \gamma$ fusion process, the hadronic decays of the $Y(4260)$, and some other processes. 
$J^{P}=0^{+}$. Their resonance parameters in units of $\mathrm{MeV}$ are $[59,60]$

$$
\begin{array}{lll}
M_{X(4500)}=4506 \pm 11_{-15}^{+12}, & \Gamma_{X(4500)}=92 \pm 21_{-20}^{+21} \\
M_{X(4700)}=4704 \pm 10_{-24}^{+14}, & \Gamma_{X(4700)}=120 \pm 31_{-33}^{+42}
\end{array}
$$

- In 2017, BESIII Collaboration presented more precise measurements of the $e^{+} e^{-} \rightarrow \pi^{+} \pi^{-} J / \psi$ cross section at the center-of-mass energy from 3.77 to $4.60 \mathrm{GeV}$ [63] and the $e^{+} e^{-} \rightarrow \pi^{+} \pi^{-} h_{c}$ cross section at the center-of-mass energy from 3.896 to $4.600 \mathrm{GeV}$ [64]. Three vector structures $Y(4220), Y(4320)$ and $Y(4390)$ were reported with the resonance parameters in units of MeV:

\begin{tabular}{c|ccc}
\hline states & mass & width & channel \\
\hline$Y(4220)$ & $4222.0 \pm 3.1 \pm 1.4$ & $44.1 \pm 4.3 \pm 2.0$ & $\pi^{+} \pi^{-} J / \psi$ \\
& $4218.4_{-4.5}^{+5.5} \pm 0.9$ & $66.0_{-8.3}^{+12.3} \pm 0.4$ & $\pi^{+} \pi^{-} h_{c}$ \\
$Y(4320)$ & $4320 \pm 10.4 \pm 7.0$ & $101.4_{-19.7}^{+25.3} \pm 10.2$ & $\pi^{+} \pi^{-} J / \psi$ \\
$Y(4390)$ & $4391.5_{-6.8}^{+6.3} \pm 1.0$ & $139.5_{-20.6}^{+16.2} \pm 0.6$ & $\pi^{+} \pi^{-} h_{c}$ \\
\hline
\end{tabular}

In fact this updated analysis of the $e^{+} e^{-} \rightarrow \pi^{+} \pi^{-} J / \psi$ cross section [64] shows that the $Y(4260)$ contains two substructures, the $Y(4220)$ and $Y(4320)$.

- The Belle Collaboration carried out an analysis of the $e^{+} e^{-} \rightarrow J / \psi D \bar{D}$ cross section, and concluded that a broad structure $X^{*}(3860)$ exists in the $D \bar{D}$ invariant mass spectrum, which has a mass $M=3862_{-32-13}^{+26+40} \mathrm{MeV}$ and width $201_{-67-82}^{+154+88} \mathrm{MeV}$ [65]. Their result shows that $J^{P C}=0^{++}$ is favored over $J^{P C}=2^{++}$at the level of $2.5 \sigma$ [65].

- A new charged structure $Z_{c}(4032)$ was reported by BESIII Collaboration in the $\psi(3860) \pi^{+}$invariant mass spectrum of the $e^{+} e^{-} \rightarrow Y(4260) \rightarrow \psi(3860) \pi^{+} \pi^{-}$process [66]. BESIII confirmed the vector charmonium-like structure $Y(4220)$ in the $\psi(3860) \pi^{+} \pi^{-}$invariant mass spectrum.

- In 2018, LHCb found the evidence of a structure in the $\eta_{c}(1 S) \pi^{-}$invariant mass spectrum of the $B^{0} \rightarrow \eta_{c}(1 S) K^{+} \pi^{-}$decay, which was named as the $X(4100)$ with a mass $M=4096 \pm 20_{-22}^{+18} \mathrm{MeV}$ and width $\Gamma=152 \pm 58_{-35}^{+60} \mathrm{MeV}$ [67]. The LHCb measurement also indicated that the $X(4100)$ structure may be described under the spin-parity $J^{P}=0^{+}$or $J^{P}=1^{+}$assignments [67].
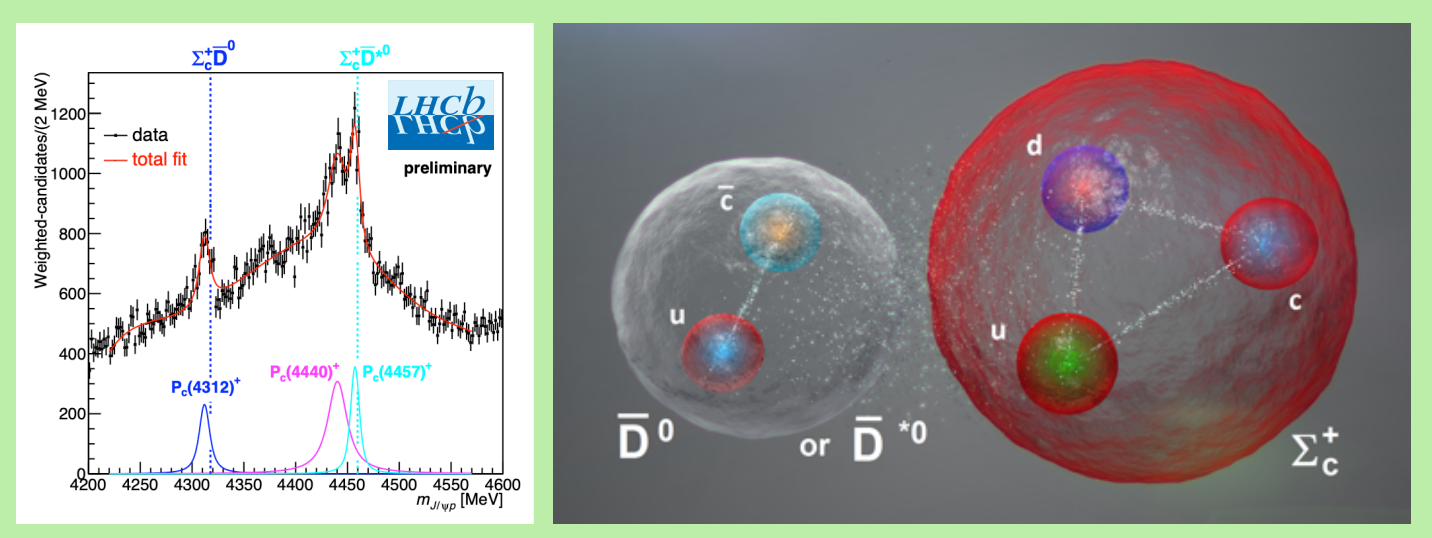

Figure 4: Three new hidden-charm pentaquarks observed by the LHCb Collaboration. Figures were taken from Ref. [68]. 
- Very recently, the LHCb Collaboration announced the observation of three new pentaquarks [68] at the Rencontres de Moriond QCD conference, as shown in Fig. 4. In the measured $J / \psi p$ invariant mass spectrum, a new pentaquark $P_{c}(4312)$ was discovered with a $7.3 \sigma$ significance. The new $\mathrm{LHCb}$ analysis further found that the $P_{c}(4450)$ is composed of two substructures $P_{c}(4440)$ and $P_{c}(4457)$ with $5.4 \sigma$ significance. Their resonance parameters are collected as following

$$
\begin{aligned}
P_{c}(4312)^{+}: \quad m & =4311.9 \pm 0.7_{-0.6}^{+6.8} \mathrm{MeV}, \\
\Gamma & =9.8 \pm 2.7_{-4.5}^{+3.7} \mathrm{MeV} \\
P_{c}(4440)^{+}: \quad m & =4440.3 \pm 1.3_{-4.7}^{+4.1} \mathrm{MeV}, \\
\Gamma & =20.6 \pm 4.9_{-10.1}^{+8.7} \mathrm{MeV}, \\
P_{c}(4457)^{+}: \quad m & =4457.3 \pm 1.3_{-4.1}^{+0.6} \mathrm{MeV}, \\
\Gamma & =6.4 \pm 2.0_{-1.9}^{+5.7} \mathrm{MeV} .
\end{aligned}
$$

The isospin of $P_{c}(4312), P_{c}(4440)$ and $P_{c}(4457)$ is $I=1 / 2$ since these three pentaquarks were discovered in the $J / \psi p$ channel. The $P_{c}(4312)^{+}$lies below the $\Sigma_{c}^{+} \bar{D}^{0}$ threshold, while the masses of the $P_{c}(4440)^{+}$and $P_{c}(4457)^{+}$are slightly lower than the $\Sigma_{c}^{+} \bar{D}^{* 0}$ threshold. The observation of $P_{c}(4312), P_{c}(4440)$ and $P_{c}(4457)$ clearly confirms the hidden-charm molecular pentaquarks.

The present situation of the charmonium-like $X Y Z$ and $P_{c}$ states is a bit similar to a) that of the ground-state mesons and baryons in 1960s, and b) that of the charmonia in 1980s. With such abundant novel phenomenon, the most important task is to identify the genuine tetraquark and pentaquark signals. There have accumulated hundreds of investigations of the tetraquark and pentaquark systems with various phenomenological models. In this review, we will introduce several typical phenomenological methods/models and their applications to multiquark states.

Our previous review focused on the hidden-charm and hidden-bottom multiquark systems [1]. In the present article, we will summarize the experimental and theoretical progress on the hidden-charm tetraquark and pentaquark states in the past three years. Besides the hidden heavy flavor systems, we shall discuss more interesting tetraquark and pentaquark systems either with open heavy flavor or with three/four heavy quarks. These new states include the exotic tetraquarks $Q Q \bar{Q} \bar{Q}$ and $Q Q \bar{q} \bar{q}$ systems where $Q$ is a heavy quark. The $Q Q \bar{Q} \bar{Q}$ states may be produced at LHC while the $Q Q \bar{q} \bar{q}$ system may be searched for at BelleII. Moreover, we will summarize theoretical predictions of the multiquark states from various formalisms such as the chromomagnetic interaction (CMI), constituent quark model, meson exchange model, heavy quark and heavy diquark symmetry, QCD sum rules, Faddeev equation for the three body systems, Skyrme model and the chiral quark-soliton model, and the lattice QCD simulations. We shall pay special attention to those theoretical schemes which are not covered or not addressed in depth in our previous review [1], such as the chromomagnetic interaction, heavy quark symmetry etc. We shall emphasize the model-independent predictions of these models which are truly/closely related to Quantum Chromodynamics (QCD).

Besides the present article and our previous review [1], there exist many nice reviews of the XYZ states in literature $[69,70,71,72,73,74,75,76]$. Interested readers are encouraged to consult them for a glimpse of this extremely active and vast field.

\section{$2 S U(6)$ symmetry and Chromomagnetic interaction (CMI)}

The hyperfine structure for atoms is induced by the spin-related interaction between electrons and nuclei. In a similar situation, the hyperfine structure in hadron spectroscopy is from the spin-related interaction between quarks or between quarks and antiquarks. In the Hamiltonian or Lagrangian formalism, such a term contains a color factor since quarks interact in the color space. The simple 
color-magnetic interaction arises from the one-gluon-exchange potential and causes the mass splittings of the conventional hadrons, whose color configuration is unique. After the inclusion of the quark mass, one obtains an effective description method for hadron masses, which is the Hamiltonian of the color-magnetic interaction or chromomagnetic interaction (CMI) model.

Although the CMI model is simple and the results may sometimes deviate from reality, it is helpful to understand the basic features of hadron spectra through the estimated masses, see Ref. [77] for detailed arguments. This type of simple models together with symmetry analysis can also be helpful to confirm dynamical models to some extent. For example, from the analysis of the mass splittings of the conventional mesons and baryons, the author of Ref. [78] concluded that the Cornell potential (Coulomb+linear) is the preferred model.

Within the multiquark systems, there may exist two or more color configurations. For example, one may decompose the fully heavy tetraquark $(Q Q)_{A}(\bar{Q} \bar{Q})_{B}$ into two clusters. There are two possible color configurations: $6_{A} \times \overline{6}_{B}$ and $\overline{3}_{A} \times 3_{B}$. The $\overrightarrow{\lambda_{i}} \cdot \overrightarrow{\lambda_{j}}$ type color-electric interactions do not induce mixing between different color configurations. In contrast, the chromomagnetic interaction will not only mix two different color configurations, but also cause the mass splitting within the multiplets.

Recent developments in spectroscopy motivated the proposal of improved CMI models or methods which are becoming powerful tools in studying interesting multiquark states. We shall discuss recent developments about the model and application methods in this section.

\subsection{Hamiltonian for the CMI model}

A realistic quark potential model generally includes the quark kinetic term, color Coulomb term, color-spin interaction term, spin-orbital term, tensor term, and color confinement term. Considering the Hamiltonian in Ref. [79] for the $S$-wave hadrons (where the sin-orbit and tensor interactions vanish) and ignoring the electromagnetic part, one has

$$
\begin{aligned}
\hat{H}= & \left\{L\left(\vec{r}_{1}, \vec{r}_{2}, \ldots\right)+\sum_{i}\left(m_{0 i}+\frac{\vec{p}_{i}^{2}}{2 m_{0 i}}\right)+\frac{1}{4} \sum_{i<j} \alpha_{s} \overrightarrow{\lambda_{i}} \cdot \overrightarrow{\lambda_{j}}\left[\frac{1}{|\vec{r}|}-\frac{\pi}{2} \delta^{3}(\vec{r})\left(\frac{1}{m_{0 i}^{2}}+\frac{1}{m_{0 j}^{2}}\right)\right.\right. \\
& \left.\left.-\frac{1}{2 m_{0 i} m_{0 j}}\left(\frac{\overrightarrow{p_{i}} \cdot \vec{p}_{j}}{|\vec{r}|}+\frac{\vec{r} \cdot\left(\vec{r} \cdot \vec{p}_{i}\right) \vec{p}_{j}}{|\vec{r}|^{3}}\right)\right]\right\}-\frac{\pi}{6} \sum_{i<j} \alpha_{s} \delta^{3}(\vec{r}) \frac{\overrightarrow{\lambda_{i}} \cdot \overrightarrow{\lambda_{j}} \vec{\sigma}_{i} \cdot \vec{\sigma}_{j}}{m_{0 i} m_{0 j}} .
\end{aligned}
$$

Here, the Gell-Mann matrix $\lambda_{i}$ should be replaced by $-\lambda_{i}^{*}$ for an antiquark, $L$ is responsible for the quark binding, $\vec{r}_{i}, \vec{p}_{i}$, and $m_{0 i}$ are the position, momentum, and constituent mass of the $i$-th (anti)quark, respectively, and $\vec{r}=\vec{r}_{i}-\vec{r}_{j}$. The average on the orbital wave function $\Psi_{0}(L=0)$ gives

$$
\begin{aligned}
H & =\left\langle\Psi_{0}|\hat{H}| \Psi_{0}\right\rangle \equiv \sum_{i} m_{i}-\sum_{i<j} C_{i j} \overrightarrow{\lambda_{i}} \cdot \overrightarrow{\lambda_{j}} \vec{\sigma}_{i} \cdot \vec{\sigma}_{j}=\sum_{i} m_{i}+H_{C M I}, \\
M & =\sum_{i=1} m_{i}+\left\langle H_{C M I}\right\rangle
\end{aligned}
$$

where $m_{i}$ containing various effects is the effective mass for the $i$ th (anti)quark and $C_{i j}=\left\langle\alpha_{s} \delta^{3}(\vec{r})\right\rangle \pi /\left(6 m_{0 i} m_{0 j}\right)$.

The coupling parameter $C_{i j}$ is also written as $v /\left(m_{i} m_{j}\right)$ in the literature. Note that $\left\langle\alpha_{s} \delta^{3}(\vec{r})\right\rangle$ or $v$ for mesons differs from that for baryons. This mass expression is also called Sakharov-Zeldovich formula $[80,78]$.

Now, the model contains only the color-spin operator and this Hamiltonian should have an $S U(6)_{c s}$ symmetry. The eigenstate of $H$ is also that of $S U(6)_{c s}$. One may express the eigenvalue on a state with Casimir operators. For a state containing only identical quarks whose flavor-spin-color wave function is $\varphi$, one has $[81,82]$

$$
\left\langle\varphi\left|-\sum_{i<j}\left(\lambda_{i} \cdot \lambda_{j}\right)\left(\sigma_{i} \cdot \sigma_{j}\right)\right| \varphi\right\rangle=8 N+\frac{4}{3} S(S+1)+2 C_{2}\left[S U(3)_{c}\right]-4 C_{2}\left[S U(6)_{c s}\right],
$$


where $N$ is the number of quarks, $S$ is the total spin, and the definitions for the quadratic Casimir operators are

$$
\begin{aligned}
& C_{2}[S U(2)]=\frac{1}{4}\left(\sum_{i}^{N} \vec{\sigma}_{i}\right)^{2}, \quad C_{2}[S U(3)]=\frac{1}{4}\left(\sum_{i}^{N} \vec{\lambda}_{i}\right)^{2}, \\
& C_{2}[S U(6)]=\frac{1}{8}\left[\frac{2}{3}\left(\sum_{i}^{N} \vec{\sigma}_{i}\right)+\left(\sum_{i}^{N} \vec{\lambda}_{i}\right)^{2}+\left(\sum_{i}^{N} \vec{\sigma}_{i} \vec{\lambda}_{i}\right)^{2}\right] .
\end{aligned}
$$

If a representation is specified by the Young diagram $\left[f_{1}, \ldots, f_{g}\right]$, one has

$$
C_{2}[S U(g)]=\frac{1}{2}\left[\sum_{i} f_{i}\left(f_{i}-2 i+g+1\right)-\frac{N^{2}}{g}\right] .
$$

For a quark-antiquark pair or two quarks without constraint from the Pauli principle, the color part and spin part can be calculated separately and the formula in this special case is

$$
\left\langle\varphi\left|\left(\lambda_{i} \cdot \lambda_{j}\right)\left(\sigma_{i} \cdot \sigma_{j}\right)\right| \varphi\right\rangle=4\left[C_{2}\left[S U(3)_{c}\right]-\frac{8}{3}\right]\left[S(S+1)-\frac{3}{2}\right] .
$$

If $C_{i j}=C$ and one acts $H_{C M I}$ on a multiquark system whose quark content is $q^{m} \bar{q}^{n}(n+m>3)$, the average value can be expressed as [83, 84]

$$
\begin{aligned}
\left\langle\varphi\left|H_{C M I}\right| \varphi\right\rangle= & C\left\{8 N+4 C_{2}\left[S U(6)_{c s}, t o t\right]-\frac{4}{3} S_{t o t}\left(S_{t o t}+1\right)-2 C_{2}\left[S U(3)_{c}, t o t\right]\right. \\
& +4 C_{2}\left[S U(3)_{c}, q\right]+\frac{8}{3} S_{q}\left(S_{q}+1\right)-8 C_{2}\left[S U(6)_{c s}, q\right] \\
& \left.+4 C_{2}\left[S U(3)_{c}, \bar{q}\right]+\frac{8}{3} S_{\bar{q}}\left(S_{\bar{q}}+1\right)-8 C_{2}\left[S U(6)_{c s}, \bar{q}\right]\right\}
\end{aligned}
$$

Now, $N=n+m$ is the total number of quarks and antiquarks. One may also consult Ref. [85] for more information.

In principle, the symmetry breaking should be considered. Then the coupling parameters can be different for different flavors and more complicated CMI expressions may be obtained (see Eq. (5) of Ref. [84]). In addition, mixing between different color-spin structures may be induced by the chromomagnetic interaction. The number of base states for a given $J^{P C}$ is generally larger than one and we need to know all the relevant CMI expressions which can be calculated with the knowledge of group theory $[86,87,88]$. After diagonalizing the obtained matrices $\left\langle H_{C M I}\right\rangle$ 's, one obtains eigenvalues of $H_{C M I}$. Alternatively, one may directly calculate the matrix elements with the constructed wave functions in color and spin spaces separately and then combine the results together [89]. If the results in the two spaces have the form

$$
\begin{aligned}
\left\langle\varphi_{k}\left|H_{C}\right| \varphi_{l}\right\rangle & =\sum_{i<j}\left\langle\varphi_{k}\left|C_{i j} \lambda_{i} \cdot \lambda_{j}\right| \varphi_{l}\right\rangle=\sum_{i<j} a_{i j}^{k l} C_{i j} \\
\left\langle\varphi_{k}\left|H_{S}\right| \varphi_{l}\right\rangle & =\sum_{i<j}\left\langle\varphi_{k}\left|C_{i j} \sigma_{i} \cdot \sigma_{j}\right| \varphi_{l}\right\rangle=\sum_{i<j} b_{i j}^{k l} C_{i j}
\end{aligned}
$$

where $k$ or $l$ is the label of a base state, one gets (See also Ref. [77])

$$
\left\langle\varphi_{k}\left|H_{C M I}\right| \varphi_{l}\right\rangle=-\sum_{i<j}\left(a_{i j}^{k l} b_{i j}^{k l}\right) C_{i j} \equiv \sum_{i<j} X_{i j}^{k l} C_{i j} .
$$




\subsection{CMI models}

In principle, the values of $m_{i}$ and $C_{i j}$ in the CMI model (4) should be different for various systems, which can be understood from the reduction procedure in getting the Hamiltonian. However, it is difficult to exactly calculate these parameters for a given system without knowing the spatial wave function. Practically, they are extracted from the masses of conventional hadrons by assuming that quark-(anti)quark interactions are the same for all the hadron systems. This assumption certainly leads to uncertainties on mass estimations. The uncertainty caused by $m_{i}$ does not allow us to give accurate multiquark masses while the uncertainty in coupling parameters has smaller effects and the mass splittings should be more reliable. To give reasonable description for hadron spectra in the CMI model, one usually uses modified mass equation based on the adopted assumptions or chooses refined parameters in calculations. In the literature, different forms of CMI Hamiltonians can be found. Here we summarize several versions of the model in studying heavy quark multiquark states.

- Models with diquark assumption. The $S$-wave correlated quark-quark state (diquark) with color $=\overline{3}$ is assumed [90, 91], where the spin of the diquark can be 1 and 0 (see Ref. [92] for a review). For the light-light diquark, the spin-0 state is more tightly bound than the spin- 1 state. For the heavy-light diquark, the difference between the spin- 1 and spin-0 states is suppressed by the heavy quark mass. The formation mechanism of tetraquark states in the diquark configuration was pictured in Ref. [93] and the hypothesis that diquarks and antidiquarks in tetraquarks are separated by a potential barrier was introduced in Ref. [94]. In this diquark approximation, the Hamiltonian for $q_{1} q_{2} \bar{q}_{3} \bar{q}_{4}$ tetraquark states has the form $[95,96]$

$$
\begin{aligned}
H= & m_{\left[q_{1} q_{2}\right]}+m_{\left[q_{3} q_{4}\right]}+2\left(\kappa_{q_{1} q_{2}}\right) \overline{3}\left(S_{q_{1}} \cdot S_{q_{2}}\right)+2\left(\kappa_{q_{3} q_{4}}\right) \overline{3}\left(S_{\bar{q}_{3}} \cdot S_{\bar{q}_{4}}\right)+2 \kappa_{q_{1} \bar{q}_{3}}\left(S_{q_{1}} \cdot S_{\bar{q}_{3}}\right) \\
& +2 \kappa_{q_{1} \bar{q}_{4}}\left(S_{q_{1}} \cdot S_{\bar{q}_{4}}\right)+2 \kappa_{q_{2} \bar{q}_{3}}\left(S_{q_{2}} \cdot S_{\bar{q}_{3}}\right)+2 \kappa_{q_{2} \bar{q}_{4}}\left(S_{q_{2}} \cdot S_{\bar{q}_{4}}\right)+2 A \vec{L} \cdot\left(\vec{S}_{q_{1} q_{2}}+\vec{S}_{\bar{q}_{3} \bar{q}_{4}}\right)+\frac{B}{2} \vec{L}(12)
\end{aligned}
$$

or the form [97]

$$
\left.H=M_{00}+\frac{1}{2} B \vec{L}^{2}-2 a \vec{L} \cdot \vec{S}+2 \kappa_{q_{1} q_{2}}^{\prime}\left(\vec{S}_{q_{1}} \cdot \vec{S}_{q_{2}}\right)+2 \kappa_{q_{3} q_{4}}^{\prime}\left(\vec{S}_{\bar{q}_{3}} \cdot \vec{S}_{\bar{q}_{4}}\right)\right] .
$$

The former (latter) Hamiltonian is for the "type-I" ("type-II") diquark-antidiquark model where orbital excitation between the diquark and the antidiquark is involved. In the type-II model, the spin-spin interactions between quark components in different diquarks are ignored and the coupling coefficients have different values from those in the type-I model.

- Model with triquark assumption. To understand the inner structure of the LHCb pentaquark states, Lebed proposed the diquark-triquark picture in Ref. [98] where the triquark is composed of a diquark and an antiquark. The diquarks from their generation are always in the color $\overline{3}$ representation [93]. This picture is different from the one proposed in Ref. [99] where the diquark in the triquark is in the color-6 representation. Another difference lies in the stability. The diquarktriquark state in Ref. [99] looks like a static molecule stabilized by a $P$-wave barrier, while the state in Ref. [98] can exist in each partial wave and can last as long as the components continue to separate. Compared with the diquark-diquark-antiquark configuration, the antiquark belongs to a compact component of the pentaquark, although the two configurations have the same color structure. This diquark-triquark picture allows the assignment consistent with LHCb data and can qualitatively explain the measured widths. The effective Hamiltonian in this diquark-triquark picture can be written in a form similar to the model (12) [100],

$$
H=m_{\delta}+m_{\theta}+H_{S S}^{\delta}+H_{S S}^{\bar{\theta}}+H_{S S}^{\delta \bar{\theta}}+H_{S L}+H_{L L},
$$

where $\delta(\bar{\theta})$ indicates the diquark (triquark) and the subscripts of $H$ mean the spin-spin, spinorbital, and orbital-orbital interactions within or between different clusters. 
- Model with the chromoelectric term. In Ref. [101], Hogaasen et al generalized the CMI model by including the chromoelectric term,

$$
H=\sum_{i} m_{i}-\sum_{i, j} A_{i j} \vec{\lambda}_{i} \cdot \vec{\lambda}_{j}-\sum_{i, j} C_{i j} \vec{\lambda}_{i} \cdot \vec{\lambda}_{j} \vec{\sigma}_{i} \cdot \vec{\sigma}_{j}
$$

where the effective quark masses have different values from those in Eq. (4) because of the nonvanishing $A_{i j}$. The contributions from the chromoelectric term were implicitly included in the model (4).

Ref. [102] adopted a modified form of (15) to discuss the masses of the doubly and triply heavy conventional baryons. The model Hamiltonian reads

$$
H=-\frac{3}{4} \sum_{i<j} m_{i j} \vec{F}_{i} \cdot \vec{F}_{j}-\sum_{i<j} v_{i j} \vec{S}_{i} \cdot \vec{S}_{j} \vec{F}_{i} \cdot \vec{F}_{j}
$$

where $F_{i}=\lambda_{i} / 2, S_{i}=\sigma_{i} / 2$, and $m_{i j}$ is the to-be-fitted mass parameter of the quark pair labeled with $i$ and $j$.

- Model motivated with a QCD-string-junction picture [103]. Noting the fact that the effective quark masses and coupling parameters determined from mesons differ from those from baryons, Karliner, Rosner, and Nussinov proposed a method to estimate hadron masses in Refs. [104, 105]. Since there is no junction (but one QCD string) for conventional mesons and one junction (and two additional QCD strings) for conventional baryons, a universal constant $S$ is added to the baryon mass expression. For tetraquark states, the number of $S$ added to mass expressions is 2 because there are two junctions (and two more QCD strings). Besides, a correction term $B$ representing additional binding is usually needed. Therefore, the mass formula in this model generally has the form

$$
M=M_{0}+x S+B+M_{H F},
$$

where $M_{0}$ is the effective quark mass term, $x$ is an integer, and $M_{H F}$ is the hyperfine splitting term.

- Method with a reference mass scale. From the explicit calculations, one finds that the model (4) usually gives higher masses for conventional hadrons (see Table 2 for a comparison). According to our studies for various multiquark systems [106, 107, 108, 109, 110, 111, 112, 113], the obtained masses with this model are also the largest values we can obtain. They should be higher than the realistic masses. Here, "realistic" means "measured" by assuming that the states do exist. These observations indicate that the attractions between quark components in this simple model are not sufficiently considered. In order to reduce the uncertainties and obtain more appropriate estimations, one may adopt an alternative form of the mass formula.

Introducing a reference system and modifying the mass formula, one gets

$$
M=\left(M_{r e f}-\left\langle H_{C M I}\right\rangle_{r e f}\right)+\left\langle H_{C M I}\right\rangle
$$

where $M_{\text {ref }}$ and $\left\langle H_{C M I}\right\rangle_{\text {ref }}$ are the physical mass of the reference system and the corresponding CMI eigenvalue, respectively. For $M_{r e f}$, one may use the mass of a reference multiquark state or use the threshold of a reference hadron-hadron system whose quark content is the same as the considered multiquark states. With this method, the problem of using extracted quark masses from conventional hadrons in multiquark systems [114] is evaded and part of missed attraction between quark components is phenomenologically compensated. Whether this manipulation gives 
results close to realistic masses or not can be tested in more studies. In fact, such an estimation method had been applied to the multiquark states many years ago [77, 115, 116].

In calculating the CMI matrix elements for the multiquark states, we consider all the color-spin configurations and the important color mixing effects [117]. When one adopts this method, more than one thresholds or scales may exist. Since the size of a multiquark state is expected to be larger than that of a conventional hadron and the distance between quark components may be larger, the attraction between quark components should be weaker. The highest masses seem to be more reasonable in this method and we use this assumption in our studies, although we cannot give a definite answer for the problem which threshold should be adopted. From the theoretical studies in recent years, it seems that the predicted masses with such a strategy are still lower than the realistic values. That is,

$$
\max \left(M_{(18)}\right)<M_{\text {realistic }}<M_{(4)}
$$

where $M_{(18)}$ and $M_{(4)}$ are obtained with Eqs. (18) and (4), respectively.

\subsection{CMI and Conventional hadrons}

The parameters in CMI models are effective masses and coupling coefficients. In the simplest CMI model (4), one may extract effective quark masses and most of the coupling parameters with the known hadrons. There are different approaches to determine them, by fitting the masses of all known hadrons, by fitting the masses of mesons and baryons differently, or by just extracting their values with parts of hadron masses. A set of the extracted effective quark masses is $m_{n}=361.7 \mathrm{MeV}(n=u, d)$, $m_{s}=540.3 \mathrm{MeV}, m_{c}=1724.6 \mathrm{MeV}$, and $m_{b}=5052.8 \mathrm{MeV}$ while the obtained coupling parameters are listed in Table 1. For the unknown $C_{c \bar{b}}$, its value was extracted with the meson masses predicted in the Godfrey-Isgur (GI) model [33]. For the unknown $C_{s \bar{s}}, C_{c c}, C_{b b}$, and $C_{c b}$, the approximation $C_{q q} / C_{q \bar{q}}=C_{n n} / C_{n \bar{n}} \approx 2 / 3[78,118]$ has been used. This approximation seems to be applicable for all the coupling parameters. The parameters presented here are consistent with those in the literature, e.g. $[95,104]$.

Table 1: Coupling parameters in units of $\mathrm{MeV}$. The value of $C_{c \bar{b}}$ is estimated with the mass splitting in the GI model [33]. The approximations $C_{c c}=k C_{c \bar{c}}, C_{b b}=k C_{b \bar{b}}, C_{c b}=k C_{c \bar{b}}$, and $C_{s \bar{s}}=C_{s s} / k$ have been adopted, where $k \equiv C_{n n} / C_{n \bar{n}} \approx 2 / 3$.

\begin{tabular}{l|l|l|l|l|l|l|l|l|l}
\hline$C_{n n}=18.4$ & $C_{n s}=12.1$ & $C_{n c}=4.0$ & $C_{n b}=1.3$ & $C_{s s}=6.5$ & $C_{s c}=4.5$ & $C_{s b}=1.2$ & $C_{c c}=3.3$ & $C_{b b}=1.8$ & $C_{c b}=2.0$ \\
$C_{n \bar{n}}=29.8$ & $C_{n \bar{s}}=18.7$ & $C_{n \bar{c}}=6.6$ & $C_{n \bar{b}}=2.1$ & $C_{s \bar{s}}=10.5$ & $C_{s \bar{c}}=6.7$ & $C_{s \bar{b}}=2.3$ & $C_{c \bar{c}}=5.3$ & $C_{b \bar{b}}=2.9$ & $C_{c \bar{b}}=3.3$ \\
\hline
\end{tabular}

When substituting these parameters into Eq. (4) for various conventional hadrons and comparing the obtained masses with experiments (Table 2), it is obvious that most theoretical masses are higher than the measured masses. This observation illustrates that the effective attraction is not appropriately considered in the model (4). To reproduce the masses of the conventional hadrons in acceptable uncertainties in the CMI model, it is necessary to adopt modified Hamiltonian by including more effects. In the mentioned CMI models, only two models, (15) or (16) and (17), are appropriate to achieve the task. Recently, with the model (17), Karliner and Rosner obtained reasonable masses for conventional mesons and baryons in Ref. [104]. The predicted mass of $\Xi_{c c}, 3627 \pm 12 \mathrm{MeV}$, is very close to that measured by LHCb, 3621.40 $\pm 0.72 \pm 0.14 \mathrm{MeV}$ [36]. With the extended CMI model whose Hamiltonian is given in Eq. (16), Weng, Chen, and Deng explored in Ref. [102] the masses of doubly and triply heavy baryons. The parameters were obtained by fitting masses of known hadrons and they can reproduce the baryon masses well. The calculated $\Xi_{c c}$ mass $(3633.3 \pm 9.3 \mathrm{MeV})$ is also close to that measured by 
LHCb. For other predicted masses, the values in these two methods are also consistent with each other. All the predicted $Q Q q$ and $Q Q Q$ masses are not far from the lattice results [119, 120], either. The studies indicate that the CMI model is still powerful once chromoelectric interactions are appropriately considered, although the model is very simple.

\section{$2.4 \quad$ CMI and $Q \bar{Q} q \bar{q}$}

Most of the observed $X Y Z$ states belong to this category. Decades ago, such type of tetraquark states had been discussed after the excited charmonium state $\psi(2 S)$ was observed. Since the observation of the $X(3872)$ in 2003, more and more heavy hadrons with exotic properties were observed and partly confirmed in the following years. If the states have explicit exotic quantum numbers, they are good tetraquark candidates. Otherwise, some of these states may be conventional heavy quarkonia which are affected heavily by the mixing effects with four-quark components. It's possible some states are molecules or compact tetraquarks with non-exotic quantum numbers. In some cases, there exist nonresonant explanations. We are still far from understanding the nature of the $Q \bar{Q} q \bar{q}$ states. Here, we first concentrate on recent theoretical studies and developments within the CMI models, especially in recent three years. One may consult studies about the $Q \bar{Q} q \bar{q}$ states before 2016 in our previous review $[1]$.

In Ref. [95], Maiani et al discussed the nature of $X(3872)$ in the "type-I" diquark-antidiquark model (12). As the $1^{++}$tetraquark state, the properties of $X(3872)$ such as its narrow width and isospin breaking in decay can be understood. Within this model, they also discussed the properties of $X(3940)$ [now called $X(3915)], D_{s}(2317), D_{s}(2460)$, and $D_{s J}(2632)$. Latter in Ref. [121], they interpreted $Y(4260)$ as the first orbital excitation of a diquark-antidiquark state $([c s][\bar{c} \bar{s}])$. In Refs. $[122,123]$, the $Z$ (4430) was identified as the first radial excitation of the tetraquark basic supermultiplet to which $X(3872)$ belongs. In Ref. [96], the authors studied the $\operatorname{cs} \bar{c} \bar{s}$ states with different $J^{P C}$ and predicted a $0^{-+}$state at $4277 \mathrm{MeV}$ decaying into $J / \psi \phi$.

Hogaasen, Richard, and Sorba discussed the nature of $X(3872)$ in the tetraquark configuration with the mass equation (4) in Ref. [124]. By introducing the annihilation term and different masses for the $u$ and $d$ quarks, they found that the $X(3872)$ may be interpreted as a $c n \bar{c} \bar{n}$ tetraquark state where the $c \bar{c}$ pair is mostly a color-octet state. This configuration can also explain the rough ratio between the $J / \psi \rho$ and $J / \psi \omega$ decay modes. However, their parameters could not be applied to the hidden-bottom tetraquark states.

Cui et al. considered the partner states of the $X(3872)$ with the mass equation (4) [88]. They determined the coupling parameter by assuming the $X(3872)$ as a $c n \bar{c} \bar{n}$ tetraquark. With the obtained parameters, the masses of all the ground states with the configurations $Q q \bar{Q} \bar{q}, Q Q \bar{q} \bar{q}$, and $Q Q \bar{Q} \bar{q}$ were estimated, where $Q=c, b$ and $q=u, d, s$.

Stancu explored the spectrum of $\operatorname{cs} \bar{c} \bar{s}$ tetraquarks with the CMI model (4) after the observation of the $Y(4140)$ [114]. The results suggest that the $Y(4140)$ could be the strange partner of $X(3872)$ with $J^{P C}=1^{++}$in a tetraquark interpretation. The later LHCb measurement for the $X(4274)$ [60] indicates that the mass splitting between the $X(4274)$ and $X(4140)$ is consistent with the CMI model prediction.

Tetraquark states were also studied with the CMI model (4) together with the possible $q q q \bar{q} \bar{q}$ hexaquark states [125]. The $X(4140)$ and $X(4350)$ can be assigned as $1^{++}$and $0^{++} \operatorname{cs} \bar{c} \bar{s}$ states, respectively.

In a schematic study of the S-wave $b \bar{b} q \bar{q}(q=u, d, s)$ tetraquarks with the CMI model (4), the authors of Ref. [126] obtained the $1^{+} b u \bar{b} \bar{d}$ or $b d \bar{b} \bar{u}$ states with masses $10612 \mathrm{MeV}$ and $10683 \mathrm{MeV}$, which are compatible with the $Z_{b}(10610)$ and $Z_{b}(10650)$.

In Refs. [127, 128], Ali et al considered the tetraquark interpretation for the bottomonium-like states $Z_{b}(10610)$ and $Z_{b}(10650)$ with $I^{G}\left(J^{P}\right)=1^{+}\left(1^{+}\right)$. They assumed that these mesons are $[b u][\bar{b} \bar{d}]$ diquarkantidiquark states produced in the decay of the $Y(10890)$ which was also assumed to be a tetraquark state $[129,130]$. Using the Hamiltonian (12) and including the meson loop effects, they were able to 
Table 2: Comparison for hadron masses measured by experiments (Ex.) [35] and calculated by using Eq. (4), where $X_{q} \equiv C_{b c}+C_{b q}+C_{c q}$ and $Y_{q} \equiv \sqrt{\left(2 C_{b c}-C_{b q}-C_{c q}\right)^{2}+3\left(C_{c q}-C_{b q}\right)^{2}}$ with $q$ being $n$ or $s$.

\begin{tabular}{|c|c|c|c|c|c|c|c|c|c|}
\hline Hadron & CMI & Th. & Ex. & (Th.-Ex.) & Hadron & CMI & Th. & Ex. & (Th.-Ex.) \\
\hline$\pi$ & $-16 C_{n \bar{n}}$ & 246.6 & 139.6 & 107 & $\rho$ & $\frac{16}{3} C_{n \bar{n}}$ & 882.3 & 775.3 & 107 \\
\hline \multirow[t]{3}{*}{ K } & $-16 C_{n \bar{s}}$ & 602.8 & 493.7 & 109 & $K^{*}$ & $\frac{16}{3} C_{n \bar{s}}$ & 1001.7 & 891.8 & 110 \\
\hline & & & & & $\omega$ & $\frac{16}{3} C_{n \bar{n}}$ & 882.3 & 782.7 & 100 \\
\hline & & & & & $\phi$ & $\frac{16}{3} C_{s \bar{s}}$ & 1136.7 & 1019.5 & 117 \\
\hline$D$ & $-16 C_{c \bar{n}}$ & 1980.7 & 1869.7 & 111 & $D^{*}$ & $\frac{16}{3} C_{c \bar{n}}$ & 2121.5 & 2010.3 & 111 \\
\hline$D_{s}$ & $-16 C_{c \bar{s}}$ & 2157.7 & 1968.3 & 189 & $D_{s}^{*}$ & $\frac{16}{3} C_{c \bar{s}}$ & 2300.6 & 2112.2 & 188 \\
\hline$B$ & $-16 C_{b \bar{n}}$ & 5380.9 & 5279.5 & 102 & $B^{*}$ & $\frac{16}{3} C_{b \bar{n}}$ & 5425.7 & 5324.7 & 101 \\
\hline$B_{s}$ & $-16 C_{b \bar{s}}$ & 5556.3 & 5366.9 & 189 & $B_{s}^{*}$ & $\frac{16}{3} C_{b \bar{s}}$ & 5605.4 & 5415.4 & 190 \\
\hline$\eta_{c}$ & $-16 C_{c \bar{c}}$ & 3364.4 & 2983.9 & 381 & $J / \psi$ & $\frac{16}{3} C_{c \bar{c}}$ & 3477.5 & 3096.9 & 381 \\
\hline$\eta_{b}$ & $-16 C_{b \bar{b}}$ & 10059.2 & 9399.0 & 660 & $\Upsilon$ & $\frac{16}{3} C_{b \bar{b}}$ & 10121.1 & 9460.3 & 661 \\
\hline$B_{c}$ & $-16 C_{\bar{c} b}$ & 6724.6 & 6274.9 & 450 & $B_{c}^{*}[33]$ & $\frac{16}{3} C_{\bar{c} b}$ & 6795.0 & & \\
\hline$N$ & $-8 C_{n n}$ & 937.9 & 938.3 & 0 & $\Delta$ & $8 C_{n n}$ & 1232.3 & 1232.0 & 0 \\
\hline$\Sigma$ & $\frac{8}{3}\left(C_{n n}-4 C_{n s}\right)$ & 1183.7 & 1189.4 & -6 & $\Sigma^{*}$ & $\frac{8}{3}\left(C_{n n}+2 C_{n s}\right)$ & 1377.3 & 1382.8 & -6 \\
\hline$\Lambda$ & $-8 C_{n n}$ & 1116.5 & 1115.7 & 1 & & & & & \\
\hline \multirow[t]{2}{*}{$\Xi$} & $\frac{8}{3}\left(C_{s s}-4 C_{n s}\right)$ & 1330.6 & 1314.9 & 16 & $\Xi^{*}$ & $\frac{8}{3}\left(C_{s s}+2 C_{n s}\right)$ & 1524.2 & 1531.8 & -8 \\
\hline & & & & & $\Omega$ & $8 C_{s s}$ & 1672.9 & 1672.5 & 0 \\
\hline$\Lambda_{c}$ & $-8 C_{n n}$ & 2300.8 & 2286.5 & 14 & & & & & \\
\hline$\Sigma_{c}$ & $\frac{8}{3}\left(C_{n n}-4 C_{c n}\right)$ & 2454.4 & 2454.0 & 0 & $\Sigma_{c}^{*}$ & $\frac{8}{3}\left(C_{n n}+2 C_{c n}\right)$ & 2518.4 & 2518.4 & 0 \\
\hline$\Xi_{c}$ & $-8 C_{n s}$ & 2529.8 & 2467.9 & 62 & & & & & \\
\hline$\Xi_{c}^{\prime}$ & $\frac{8}{3}\left(C_{n s}-2 C_{c n}-2 C_{c s}\right)$ & 2613.5 & 2577.4 & 36 & $\Xi_{c}^{*}$ & $\frac{8}{3}\left(C_{n s}+C_{c n}+C_{c s}\right)$ & 2681.5 & 2645.5 & 36 \\
\hline$\Omega_{c}$ & $\frac{8}{3}\left(C_{s s}-4 C_{c s}\right)$ & 2774.5 & 2695.2 & 79 & $\Omega_{c}^{*}$ & $\frac{8}{3}\left(C_{s s}+2 C_{c s}\right)$ & 2846.5 & 2765.9 & 81 \\
\hline$\Lambda_{b}$ & $-8 C_{n n}$ & 5629.0 & 5619.6 & 9 & & & & & \\
\hline$\Sigma_{b}$ & $\frac{8}{3}\left(C_{n n}-4 C_{b n}\right)$ & 5811.4 & 5811.3 & 0 & $\Sigma_{b}^{*}$ & $\frac{8}{3}\left(C_{n n}+2 C_{b n}\right)$ & 5832.2 & 5832.1 & 0 \\
\hline$\Xi_{b}$ & $-8 C_{n s}$ & 5858.0 & 5794.5 & 64 & & & & & \\
\hline$\Xi_{b}^{\prime}$ & $\frac{8}{3}\left(C_{n s}-2 C_{b n}-2 C_{b s}\right)$ & 5973.7 & 5935.0 & 39 & $\Xi_{b}^{*}$ & $\frac{8}{3}\left(C_{n s}+C_{b n}+C_{b s}\right)$ & 5993.7 & 5955.3 & 39 \\
\hline$\Omega_{b}$ & $\frac{8}{3}\left(C_{s s}-4 C_{b s}\right)$ & 6137.9 & 6046.1 & 92 & $\Omega_{b}^{*}$ & $\frac{8}{3}\left(C_{s s}+2 C_{b s}\right)$ & 6157.1 & & \\
\hline$\Xi_{c c}$ & $\frac{8}{3}\left(C_{c c}-4 C_{c n}\right)$ & 3777.0 & 3621.4 & 156 & $\Xi_{c c}^{*}$ & $\frac{8}{3}\left(C_{c c}+2 C_{c n}\right)$ & 3841.0 & & \\
\hline$\Omega_{c c}$ & $\frac{8}{3}\left(C_{c c}-4 C_{c s}\right)$ & 3950.2 & & & $\Omega_{c c}^{*}$ & $\frac{8}{3}\left(C_{c c}+2 C_{c s}\right)$ & 4022.2 & & \\
\hline$\Xi_{b b}$ & $\frac{8}{3}\left(C_{b b}-4 C_{b n}\right)$ & 10458.2 & & & $\Xi_{b b}^{*}$ & $\frac{8}{3}\left(C_{b b}+2 C_{b n}\right)$ & 10479.0 & & \\
\hline$\Omega_{b b}$ & $\frac{8}{3}\left(C_{b b}-4 C_{b s}\right)$ & 10637.9 & & & $\Omega_{b b}$ & $\frac{8}{3}\left(C_{b b}+2 C_{b s}\right)$ & 10657.1 & & \\
\hline$\Xi_{b c}$ & $\frac{8}{3}\left(-X_{n}-Y_{n}\right)$ & 7106.6 & & & & & & & \\
\hline$\Xi_{b c}^{\prime}$ & $\frac{8}{3}\left(-X_{n}+Y_{n}\right)$ & 7132.4 & & & $\Xi_{b c}^{*}$ & $\frac{8}{3} X_{n}$ & 7158.7 & & \\
\hline$\Omega_{b c}$ & $\frac{8}{3}\left(-X_{s}-Y_{s}\right)$ & 7281.2 & & & & & & & \\
\hline \multirow[t]{3}{*}{$\Omega_{b c}^{\prime}$} & $\frac{8}{3}\left(-X_{s}+Y_{s}\right)$ & 7312.9 & & & $\Omega_{b c}^{*}$ & $\frac{8}{3} X_{s}$ & 7338.3 & & \\
\hline & & & & & $\Omega_{c c c}$ & $8 C_{c c}$ & 5200.0 & & \\
\hline & & & & & $\Omega_{b b b}$ & $8 C_{b b}$ & 15172.7 & & \\
\hline$\Omega_{c c b}$ & $\frac{8}{3}\left(C_{c c}-4 C_{b c}\right)$ & 8489.0 & & & $\Omega_{c c b}^{*}$ & $\frac{8}{3}\left(C_{c c}+2 C_{b c}\right)$ & 8521.6 & & \\
\hline$\Omega_{b b c}$ & $\frac{8}{3}\left(C_{b b}-4 C_{b c}\right)$ & 11813.2 & & & $\Omega_{b b c}^{*}$ & $\frac{8}{3}\left(C_{b b}+2 C_{b c}\right)$ & 11845.8 & & \\
\hline
\end{tabular}


reproduce their measured masses [35].

After the observation of the $Z_{c}(3900)$, Maiani et al interpreted it as the tetraquark state around $3880 \mathrm{MeV}$ [131], which was predicted in their previous study within the diquark-antidiquark model (12) [95]. They also discussed the possibility of another peak around $3760 \mathrm{MeV}$.

In Ref. [101], Hogaasen et al used the Hamiltonian (15) to discuss possible partner states of the $X(3872)$. Their fitted parameters can reproduce the mass spectrum of the $c \bar{c} n \bar{n}$ states obtained with Eq. (4) in Ref. [124]. The $1^{+} b \bar{b} n \bar{n}$ states $(I=0$ and $I=1)$ may lie around $10.62 \mathrm{GeV}$, and the $Z_{b}(10610)^{ \pm}$or $Z_{b}(10650)^{ \pm}$is such a tetraquark candidate, whose $(b \bar{b})$ component is almost a color-octet state. They also identified the $Z_{c}(3900)$ implied in Ref. [124] as the corresponding $c n \bar{c} \bar{n}$ tetraquark.

After the development of meson spectroscopy for about ten years, many candidates of exotic hadrons were reported. To understand their possible natures, the "type-II" model (13) was proposed in Ref. [97]. According to this model, the $X(3872)$ is still the state with the flavor wave function $[c n]_{S=1}[\bar{c} \bar{n}]_{S=0}+$ $[c n]_{S=0}[\bar{c} \bar{n}]_{S=1}$; the other combination $[c n]_{S=1}[\bar{c} \bar{n}]_{S=0}-[c n]_{S=0}[\bar{c} \bar{n}]_{S=1}$ and $[c n]_{S=1}[\bar{c} \bar{n}]_{S=1}$ can form the $Z_{c}(3900)$ and $Z_{c}(4020)$ with $J^{P}=1^{+}$; the $X(3915)$ and $X(3940)$ are identified to be $[c n]_{S=1}[\bar{c} \bar{n}]_{S=1}$ with $J^{P}=0^{+}$and $[c n]_{S=1}[\bar{c} \bar{n}]_{S=1}$ with $J^{P}=2^{+}$, respectively. Within these assignments, the quark-quark interactions are assumed to be stronger while the quark-antiquark interactions are negligible, which implies that the assumed diquarks in tetraquarks are more compact than those in conventional baryons. In the $P=-$ case, six exotic vector states can be assigned: the $Y(4260)$ has the same spin structure and spin interactions as the $X(3872)$ but has an additional $P$-wave excitation between the diquark and the antidiquark, the $Y(4008)$ is the orbitally excited $[c n]_{S=0}[\bar{c} \bar{n}]_{S=0}$, the $Y(4630)$ is the $S_{t o t}=2 P$-wave state, the $Y(4290)$ or $Y(4220)$ is identified as the orbitally excited $S_{t o t}=0[c n]_{S=1}[\bar{c} \bar{n}]_{S=1}$ state with $J^{P C}=1^{--}$, and the $Y(4660)$ and $Y(4360)$ are the first radial excitations of $Y(4260)$ and $Y(4008)$, respectively. The observed decay $Y(4260) \rightarrow \gamma X(3872)$ was argued to be a natural consequence of the diquark-antidiquark description for these two states [132].

The authors of Ref. [133] assumed the $[Q q]_{\overline{3}_{c}}[\bar{Q} \bar{q}]_{3_{c}}$ diquark-antidiquark configuration. Noticing the similar color structure between the assumed diquark-antidiquark system and the quark-antiquark system, the authors estimated the masses in a similar way,

$$
m_{(q \bar{q})}^{J}=m_{q}+m_{\bar{q}}+J(J+1) \Delta,
$$

where $q$ can also be a scalar or axialvector diquark. After considering the mass shifts due to the recombined meson-meson rescattering effects, they presented the masses of $[c n][\bar{c} \bar{n}],[c s][\bar{c} \bar{s}]$, and $[c n][\bar{c} \bar{s}]$ tetraquark states.

After assuming the $X(3872)$ as a $1^{++}$diquark-antidiquark type tetraquark and using it as an input, the authors of Ref. [134] investigated possible assignments for its partner states through the mass splittings calculated with the CMI method (18). The masses of two states with $1^{+-}$and one state with $2^{++}$fit very nicely to the $X(3823), X(3900)$, and $X(3940)$.

In Ref. [135], Lebed and Polosa proposed that the $\chi_{c 0}(3915)$ [originally called $Y(3940)$, now called $X(3915)]$ is the lightest $0^{++} c \bar{c} s \bar{s}$ diquark-antidiquark state in the "type-II" model (13). This identification may explain its observed width around $20 \mathrm{MeV}$. After treating the $Y(4140)$ as a $1^{++} c \bar{c} s \bar{s}$ state and the $Y(4230)$ and $Y(4360)$ as $1^{--} c \bar{c} s \bar{s}$ states, they further interpreted the $Y(4008)$ and $Y(4660)$ as $c \bar{c} s \bar{s}$ states. Moreover, the $X(4350)$ is interpreted as a $0^{++}$or $2^{++} c \bar{c} s \bar{s}$ tetraquark state, while the $Y(4274)$ may be an orbitally excited $c \bar{c} s \bar{s}$ state. In 2006 the LHCb reported other two exotic structures in the $J / \psi \phi$ channel [60], the $X(4500)$ and $X(4700)$. In Ref. [136] Maiani et al assigned the $Y(4140)$ as the $1^{++}$ ground tetraquark, and the $X(4500)$ and $X(4700)$ as two radially excited diquark-antidiquark states. They noticed that the $X(4274)$ corresponds to two almost degenerate states with $J^{P C}=0^{++}$and $2^{++}$. Predictions of the other $[c s][\bar{c} \bar{s}]$ states within this picture will be tested by upcoming experiments.

Later Zhu et al presented a study on the hidden charm tetraquarks in the $[c q]_{\overline{3}_{c}}[\bar{c} \bar{q}]_{3_{c}}$ diquarkantidiquark model (12) [137]. The authors proposed the following assignments: the $Z_{c}(3900)$ with $J^{P}=1^{+}$can be explained as $[c n]_{\overline{3}_{c}}[\bar{c} \bar{n}]_{3_{c}}$, the $Z_{c}(4020)$ with $J^{P}=1^{+}$is a companion of $Z_{c}(3900)$, the 
$Z(4430)$ is the radial excitation of $Z_{c}(3900)$, the $X(4140)$ and $X(4274)$ may be the $1^{+}$tetraquarks with the flavor wave function $(u \bar{u}+d \bar{d}-2 s \bar{s}) c \bar{c}$, the $X(4500)$ and $X(4700)$ may be the radial excitations of the $0^{+}$tetraquarks with $(u \bar{u}+d \bar{d}+s \bar{s}) c \bar{c}$ and $(u \bar{u}+d \bar{d}-2 s \bar{s}) c \bar{c}$ respectively, and the $Y(4260)$ may be an orbitally excited diquark-antidiquark tetraquark.

In Ref. [138], Ali et al studied the $J^{P C}=1^{--} Y$ tetraquark states with an extended Hamiltonian of the model (13) by including the tensor coupling contributions. Only two scenarios are consistent with the Belle, BaBar, and BESIII data in the diquark-antidiquark picture. Each scenario contains four $Y$ states. Scenario I contains $Y(4008), Y(4260), Y(4360)$, and $Y(4660)$, while Scenario II contains $Y(4220), Y(4330), Y(4390)$, and $Y(4660)$.

In Ref. [106], we updated estimations for the mass spectrum of the $c s \bar{c} \bar{s}$ tetraquark states with the CMI method (18). After assuming the $X(4140)$ to be the lowest $J^{P C}=1^{++} \operatorname{cs} \bar{c} \bar{s}$ state, we confirmed the mass splittings obtained in Ref. [114]. We found that the $X(4274)$ should be the other $1^{++} \operatorname{cs} \bar{c} \bar{s}$ tetraquark, and the $X(4350)[139]$ is probably a $0^{++} c s \bar{c} \bar{s}$, while the interpretation of the $X(4500)$ and $X(4700)$ needs orbital or radial excitations. In this study we also confirmed the mass inequality (19), and found that $M_{\text {realistic }}$ is closer to $\max \left(M_{(18)}\right)$ than to $M_{(4)}$. Similar features may be found in other systems.

In Ref. [113], we have systematically evaluated the mass spectra of the $Q_{1} q_{2} \bar{Q}_{3} \bar{q}_{4}(Q=c, b$ and $q=u, d, s)$ states. Besides the estimation method (18), we also roughly estimated the tetraquark mass spectra by treating the mass of the $X(4140)$ as an input. The quark mass differences $m_{s}-m_{n}$ and $m_{b}-m_{c}$ need to be replaced by other values, since quarks get different masses in different systems. With this replacement method, higher masses than $\max \left(M_{(18)}\right)$ can be obtained. Such masses satisfy the inequality (19) and we treat them as realistic values. We show the results for $c n \bar{c} \bar{n}$ states in Fig. 5. Results for other systems can also be found in this study [113]. It is possible to assign both the $Z_{c}(4100)$ and $X(3860)$ to be the $0^{++} c n \bar{c} \bar{n}$ tetraquark states. With the defined measure reflecting effective quark interactions (see Sec. 2.12), we argued that this assignment is acceptable. Another possible tetraquark candidate is the $Z_{c}(4200)$, but we need further studies to answer whether the assigned structures are correct or not. At present, our results with this updated method are higher than the masses obtained in an early paper [77] where the $c n \bar{c} \bar{n}, b n \bar{b} \bar{n}$, and $c n \bar{b} \bar{n}$ states were all found to be stable. The $J=2$ $c n \bar{c} \bar{s}$ and $b n \bar{b} \bar{s}$ are also weakly bound, although the binding energy in the $J=2$ sector is very weak.

\subsection{CMI and $Q Q \bar{q} \bar{q}$}

The study of the doubly heavy tetraquark states $Q Q \bar{q} \bar{q}(Q=c, b, q=u, d, s)$ has a long history in the literature. Various approaches have been applied to such systems, and the most interesting state is the lowest isoscalar $1^{+} c c \bar{u} \bar{d}$ (or its antiparticle $u d \bar{c} \bar{c}$ ), named $T_{c c}$. The $T_{c c}$ and its partner tetraquark states are explicitly exotic. They do not suffer from possible annihilation effects and their inner quark interactions may provide enough attractions to stabilize the four-quark system. One may consult Refs. $[140,141,142,143,144,145,77,146,147,148,149,150,151,125,152,153,154,155,156,157,158]$ for relevant investigations before 2003. Whether they are bound or not depends on models and adopted approximations. Generally speaking, the lowest $I\left(J^{P}\right)=0\left(1^{+}\right)$states are narrow tetraquarks. Up to now, neither the $T_{c c}$ nor any of its partner states have been observed in experiments. Recently, the confirmation of the $\Xi_{c c}$ state reignited interests in these exotic states since the $Q Q q$ and $Q Q \bar{q} \bar{q}$ systems may be related to each other with the heavy quark symmetry (see the following Sec. 5). In this subsection, we mainly concentrate on recent developments in studies with CMI models since 2003.

In Ref. [88], Cui et al investigated the mass spectrum of the $Q Q \bar{q} \bar{q}$ tetraquark states with Eq. (4) using the mass of $X(3872)$ as input. The obtained mass of the most interesting $T_{c c}$ state with $I\left(J^{P}\right)=0\left(1^{+}\right)$is $3786 \mathrm{MeV}$. This value is about $100 \mathrm{MeV}$ below the $D D^{*}$ threshold and thus this state should be narrow. The $I\left(J^{P}\right)=0\left(1^{+}\right) T_{b b}$ is also a stable state. The results in Ref. [125] obtained with Eq. (4) indicate that the lowest $1^{+} T_{c c}$ state is also below the $D D^{*}$ threshold. 


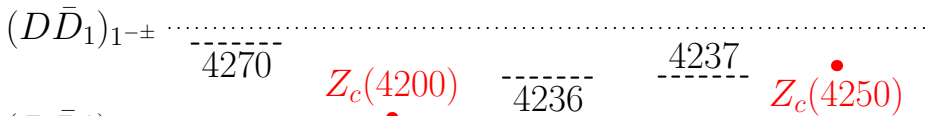

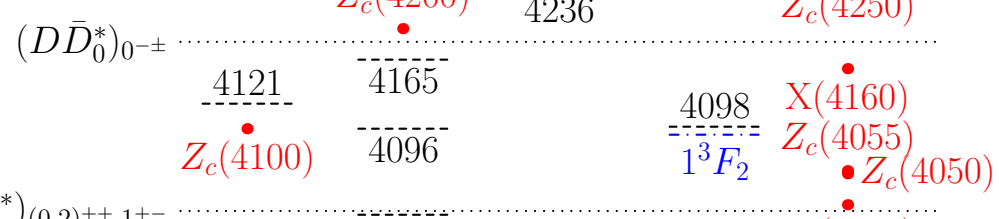

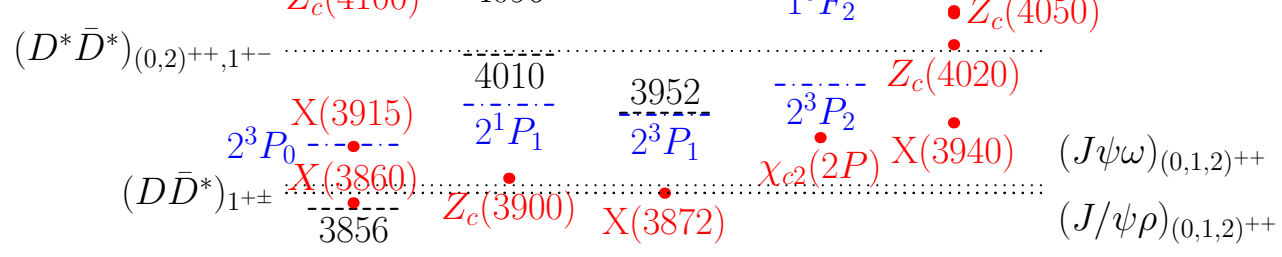

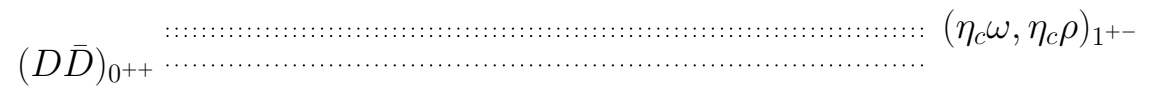

$$
\begin{aligned}
& 3597 \\
& \begin{array}{ccc}
h_{c} & \frac{\chi_{c 2}}{\chi_{3475}} \\
1^{1} P_{1} & \frac{\chi_{c 1}}{1^{3} P_{1}} & 1^{3} P_{2}^{2}
\end{array} \\
& \frac{-\cdots \cdot}{\chi_{c 0}} 1^{3} P_{0} \\
& (J / \psi \pi)_{1^{+-}} \\
& \begin{array}{ccccc}
0^{++} & 1^{+-} & 1^{++} & 2^{++} & ? ? ?
\end{array}
\end{aligned}
$$

Figure 5: Relative positions for the $c n \bar{c} \bar{n}$ tetraquarks (black dashed lines), quark model predictions for the charmonia (blue dash-dotted lines), observed charmonia (red solid lines), states with exotic properties (red solid dots), and various meson-meson thresholds (black dotted lines). The masses are given in units of $\mathrm{MeV}$. The subscripts of threshold symbols are $J^{P C}$ in the $S$-wave case. Note the results are the same for the $c n \bar{c} \bar{n}$ tetraquark states with $I=0$ and $I=1$. Taken from Ref. [113]. 
In Refs. $[159,160]$, Lee et al argued that the $I\left(J^{P}\right)=0\left(1^{+}\right) T_{c c}, T_{b b}$, and $T_{c b}$ states may be stable against rearrangement decays by using a diquark model with Eq. (18). In Refs. [161, 162], the masses of two $I\left(J^{P}\right)=0\left(1^{+}\right) T_{c c}$ states were estimated using the model (18), one with the color structure $[c c]_{3_{c}}[\bar{u} \bar{d}]_{3_{c}}$ and the other with $[c c]_{6_{c}}[\bar{u} \bar{d}]_{\overline{6}_{c}}$. The lower state is $71 \mathrm{MeV}$ below the $D D^{*}$ threshold and the higher is $54 \mathrm{MeV}$ above the $D D^{*}$ threshold. Their productions in $e^{+} e^{-}$collisions were also investigated.

We have performed a systematic investigation of the $q q \bar{Q} \bar{Q}(Q=c, b, q=u, d, s)$ states with the CMI model (18) in Ref. [109], where the mixing effects of different color-spin structures were included. Considering the uncertainties in the estimation method, the mass of the $I\left(J^{P}\right)=0\left(1^{+}\right) u d \bar{c} \bar{c}$ state should be larger than $3780 \mathrm{MeV}$ but not exceed $4007 \mathrm{MeV}$, that of $u d \bar{b} \bar{b}$ should be between $10483 \mathrm{MeV}$ and $10686 \mathrm{MeV}$, that of the lowest $I\left(J^{P}\right)=0\left(0^{+}\right) u d \bar{c} \bar{b}$ should be between $7041 \mathrm{MeV}$ and $7256 \mathrm{MeV}$, and that of the lowest $0\left(1^{+}\right) u d \bar{c} \bar{b}$ should be between $7106 \mathrm{MeV}$ and $7321 \mathrm{MeV}$. Most theoretical predictions fell into these ranges, see comparison tables in Refs. [163, 109]. Our results indicate that the mixing effects for the $u d \bar{c} \bar{c}$ systems are not so significant. If our lower bounds on the masses were reasonable, the $T_{c c / b b / c b}^{I=0}$ and $n s \bar{c} \bar{c} / n s \bar{b} \bar{b} / n s \bar{c} \bar{b}$ states with $J^{P}=1^{+}$as well as $T_{c b}^{I=0}$ and $n s \bar{c} \bar{b}$ with $J^{P}=0^{+}$are probably stable. Our results for $u d \bar{Q}_{1} \bar{Q}_{2}(Q=c, b)$ are consistent with an early study performed in Ref. [77]. Namely, such states with $I\left(J^{P}\right)=0\left(1^{+}\right)$are bound. The binding results in these references [77, 109] for the $u d \bar{c} \bar{b}$ with $I\left(J^{P}\right)=0\left(0^{+}\right)$and $u s \bar{b} \bar{b}$ with $I\left(J^{P}\right)=1 / 2\left(1^{+}\right)$are also consistent. If the mass of the realistic $T_{c c}^{I=0}$ satisfies the inequality (19) and is closer to $3780 \mathrm{MeV}$ than to $4007 \mathrm{MeV}$, this tetraquark seems to be around the $D D^{*}$ threshold, a situation similar to the $X(3872)$.

Two investigations based on heavy quark symmetry analysis also show the possible existence of the doubly heavy tetraquark states $[164,165]$, which are partly motivated by LHCb observation of the double-charm baryon $\Xi_{c c}(3621)$ [36] since the role of the $c c$ diquark within $T_{c c}$ is similar to that in $\Xi_{c c}(3621)$. Later, we will further introduce this issue in Sec. 5.

Shortly after the LHCb observation of the $\Xi_{c c}(3621)$ [36], also motivated by the success of the model (17) in predicting the $\Xi_{c c}$ mass [104], Karliner and Rosner investigated the masses of the $c c \bar{u} \bar{d}, b b \bar{u} \bar{d}$, and $b c \bar{u} \bar{d}$ in Ref. [166]. They noticed that the $I\left(J^{P}\right)=0\left(1^{+}\right) c c \bar{u} \bar{d}$ is just about $7 \mathrm{MeV}$ above the $D^{0} D^{*+}$ threshold, the $I\left(J^{P}\right)=0\left(1^{+}\right) b b \bar{u} \bar{d}$ is about $215 \mathrm{MeV}$ below the $B^{-} \bar{B}^{* 0}$ threshold, and the lowest $I\left(J^{P}\right)=0\left(0^{+}\right) b c \bar{u} \bar{d}$ is $11 \mathrm{MeV}$ below the $\bar{B}^{0} D^{0}$ threshold. Therefore, they concluded that there are no stable $c c \bar{u} \bar{d}$. But there are definitely stable $b b \bar{u} \bar{d}$ states. The lifetime of the $b b \bar{u} \bar{d}$ was estimated to be 367 fs.

In the diquark-antidiquark model (12), the authors of Ref. [167] studied the spectra and decay properties of the $q q \bar{c} \bar{c}(q=u, d, s)$ states. For the lowest $u d \bar{c} \bar{c}$, its quantum numbers are $J^{P}=1^{+}$and its mass is $3.6 \mathrm{GeV}$. This state is $140(270) \mathrm{MeV}$ below the $D D\left(D D^{*}\right)$ threshold. For the other $P=+$ $u d \bar{c} \bar{c}$ states, the masses are $3.94 \mathrm{GeV}, 3.97 \mathrm{GeV}$, and $4.04 \mathrm{GeV}$ for the $0^{+}, 1^{+}$, and $2^{+}$, respectively. The masses of the $1^{-} u d \bar{c} \bar{c}$ states range between $3.82 \mathrm{GeV}$ and $4.14 \mathrm{GeV}$. The masses of their partner states, $n s \bar{c} \bar{c}$ and $s s \bar{c} \bar{c}$, can also be found in this work. In addition to the study of the mass spectrum, the authors discussed their $(q \bar{c})-(q \bar{c})$ meson-meson and $(\bar{c} \bar{q} \bar{q})-(q q q)$ antibaryon-baryon decay modes, too. However, their subsequent calculation with Eq. (4) gives an above-threshold $T_{c c \bar{u} \bar{d}}$ and a below-threshold $T_{b b \bar{u} \bar{d}}$ by adopting the diquark-antidiquark configuration [168].

\subsection{CMI and $Q Q \bar{Q} \bar{Q}$}

Generally speaking, many $X Y Z$ exotic states have been observed in particle experiments, some of which are good tetraquark candidates, as illustrated in the hidden-heavy case. However, it is still difficult to distinguish the compact multiquark picture from the molecular picture once two or more light quarks are involved in the states. For the full-heavy $Q Q \bar{Q} \bar{Q}(Q=c, b)$ states, there does not exist appropriate binding mechanism if they are treated as loosely bound molecules. If such a state is observed, it can be identified as a compact tetraquark state bound by short-range gluon-exchange interactions. In the literature, one may also find lots of theoretical studies on the heavy-full tetraquark 
states with various methods, such as MIT bag model and quark potential model [169, 170, 171, 172, 173, 140, 174, 175, 141, 142, 143, 176, 77, 148]. Similar to the $T_{c c}$ studies, controversial conclusions on their existence were drawn when different models were applied. In recent years, experimental developments in search of exotic states reignited our interests in the fully heavy tetraquark states. Here, we focus on recent theoretical studies on the $Q Q \bar{Q} \bar{Q}$ states with CMI models.

In Refs. [177, 178], the mass spectra of the diquark-antidiquark $[c c][\bar{c} \bar{c}],[b b][\bar{b} \bar{b}]$, and $[b c][\bar{b} \bar{c}]$ states were studied with the Hamiltonian (12). The authors determined the coupling parameters by solving the nonrelativistic Schrödinger equation [179]. The lowest $c c \bar{c} \bar{c}$ and $b c \bar{b} \bar{c}$ with $J^{P C}=2^{++}$were found to be above the $J / \psi J / \psi$ and $\Upsilon J / \psi$ thresholds, respectively. But the lowest states with other quantum numbers are all below relevant meson-meson thresholds. These states may be stable.

In Ref. [110], we have systematically investigated the mass spectrum of the $Q Q \bar{Q} \bar{Q}(Q=c, b)$ compact tetraquark states with the method (18). Our estimated values are lower bounds on realistic tetraquark masses, and it seems that no stable $Q Q \bar{Q} \bar{Q}$ states exist. From our study, the only possible states, which are stable or relatively narrow, are $b b \bar{b} \bar{c}$ and $b c \bar{b} \bar{c}$. The unbinding result for the $c c \bar{b} \bar{b}$ states is consistent with an early CMI calculation in Ref. [77]. Our conclusions are consistent with those drawn from dynamical studies within constituent quark models (see Sec. 3). For example, the authors of Ref. [180] concluded that no narrow bound $Q Q \bar{Q} \bar{Q}$ states are expected in experiments. For comparison, their mass values also satisfy the mass inequality (19). Since their masses are closer to our $\max \left(M_{(18)}\right)$ than to $M_{(4)}$, which feature is similar to the $c s \bar{c} \bar{s}$ case, the results in this potential calculation are probably closer to the realistic case.

In Ref. [105], Karliner et al studied the $Q Q \bar{Q} \bar{Q}(Q=c, b)$ states with the model (17) motivated by the QCD-string-junction picture in the diquark-antidiquark configuration. The predicted lowest-lying $c c \bar{c} \bar{c}$ state with $J^{P C}=0^{++}$has a mass $6192 \pm 25 \mathrm{MeV}$, which is just below the $J / \psi J / \psi$ threshold but can decay to $\eta_{c} \eta_{c}$. The predicted lowest $b b \bar{b} \bar{b}$ has a mass $18826 \pm 25 \mathrm{MeV}$, which is just $28 \mathrm{MeV}$ above the $\eta_{b} \eta_{b}$ threshold and may have a width narrow enough to be observed.

Last year, LHCb searched for a possible exotic meson $X_{b \bar{b} b \bar{b}}$ in the $\Upsilon(1 S) \mu^{+} \mu^{-}$invariant mass distribution in Ref. [181]. Such a study was motivated by theoretical predictions and by the observation of $\Upsilon \Upsilon$ production by CMS [182]. However, no significant excess was found in the mass range between 17.5 $\mathrm{GeV}$ and $20 \mathrm{GeV}$. The authors of Ref. [183] studied a possible $b \bar{b} \bar{b}$ di-bottomonium with the diquarkantidiquark picture (13). The obtained mass is approximately $100 \mathrm{MeV}$ below the $\Upsilon \Upsilon$ threshold. They argued that the decay of this state into $\Upsilon \mu \mu$ is unlikely to be observed at LHC, which is consistent with the LHCb experiment [181].

\section{7 $\quad$ CMI and $Q Q \bar{Q} \bar{q}$}

If the $Q Q \bar{Q} \bar{Q}$ states do exist, the gluon-exchange interactions would provide enough binding forces for heavy quarks, and such interactions could also bind three heavy quarks and one light quark into compact $Q Q \bar{Q} q(Q=c, b, q=u, d, s)$ states. In such systems, no long-range particle-exchange force exists, neither. Among these states, the $c c \bar{b} \bar{q}$ and $b b \bar{c} \bar{q}$ tetraquarks are explicitly exotic. Although the $Q c \bar{c} \bar{q}$ or $Q b \bar{b} \bar{q}$ tetraquarks look like the excited $D, B, D_{s}$, or $B_{s}$ mesons, their excitation energy may not be explained by simple orbital or radial excitations if such states were observed in the future experiments. Similar arguments were used in the prediction of the hidden-charm pentaquarks [184]. Therefore, the exotic tetraquark nature of the $Q Q \bar{Q} q$ would be easily identified once observed. Up to now, such systems are poorly studied.

More than twenty years ago, Silvestre-Brac investigated various tetraquark systems with the CMI method (18) [77]. For the $Q Q \bar{Q} \bar{q}$ systems, no bound states with $J=0$ but weakly bound $c c \bar{b} \bar{n}, b c \bar{b} \bar{n}$, and $b c \bar{c} \bar{n}$ states with $J=2$ were found.

In addition to the $Q q \bar{Q} \bar{q}$ and $Q Q \bar{q} \bar{q}$ states, the authors of Ref. [88] also presented results for the $Q Q \bar{Q} \bar{q}$ states using the mass equation (4). From the obtained masses, many states, e.g. the $J^{P}=2^{+}$ 
$q c \bar{c} \bar{c}, q b \bar{c} \bar{c}$, and $q c \bar{b} \bar{b}$, were found to be below their respective lowest threshold of rearrangement decays and should be narrow.

We have performed a systematic study of the $Q Q \bar{Q} \bar{q}$ systems in Ref. [107] with the CMI method (18). Our results indicate that the lowest tetraquarks are only near-threshold bound or resonant states. Since our estimated values are just lower bounds on their realistic masses, all such tetraquarks are probably not stable. However, there may exist relatively narrow and thus detectable states such as $b c \bar{b} \bar{q}$ and $b c \bar{c} \bar{q}$ if the interactions inside the diquarks are attractive, although they are above the thresholds for the rearrangement decay channels.

\subsection{CMI and $Q \bar{Q} q q q$}

In 2015, LHCb observed two pentaquark-like resonances $P_{c}(4380)$ and $P_{c}(4450)$ with opposite $P$ parities in the decay $\Lambda_{b}^{0} \rightarrow J / \psi p K^{-}$[185]. After that, in Ref. [186], the LHCb collaboration further applied a model-independent method to the same sample and the result supports their previous model-dependent observation. Later in Ref. [187], a full amplitude analysis of $\Lambda_{b}^{0} \rightarrow J / \psi p \pi^{-}$decays also supports the evidence for exotic hadron contributions from the $P_{c}(4380), P_{c}(4450)$, and $Z_{c}(4200)$. Besides the LHCb, the CLAS12 at JLab may be a possible place to observe the $P_{c}$ states in $J / \psi$ photoproduction $[188,189]$. Its spin and photocouplings may be measured with the future data, too. Now, the search for the LHCb pentaquark in the photo-production process in Hall $\mathrm{C}$ at Jefferson Lab has been approved $[190,191]$. In future, these two $P_{c}$ states and their partner states can also be searched for by PANDA/FAIR [192], EIC, and JPARC, etc.

Before the observation of the LHCb $P_{c}$ states, many studies had been performed on the existence of possible hidden-charm pentaquarks $[184,193,194,195,196,197,198,199,200,201,202,203,204]$. Here we show several examples. In Refs. [184, 193], Wu et al predicted the hidden-charm $N^{*}$ and $\Lambda^{*}$ resonances above $4 \mathrm{GeV}$ in the molecule picture. In studying the predicted nnnce and $u d s c \bar{c}$ in Ref. [196], the authors adopted three kinds of quark-quark hyperfine interactions, the flavor-spin interaction based on the meson exchange, the CMI based on one-gluon exchange, and the instanton-induced interaction based on the non-perturbative QCD vacuum structure. In Ref. [200], the discovery potential of the hidden-charm baryon resonances via photoproduction was investigated. In Ref. [204], the effects of the hidden charm $N^{*}$ in the $\pi^{-} p \rightarrow D^{-} \Sigma_{c}^{+}$reaction near threshold were discussed. In Ref. [205], there were some discussions about meson-baryon type hidden-charm pentaquarks with the help of flavor $S U(4)$ symmetry. After the LHCb observation, more and more studies appear on the nature of the $P_{c}$ states and relevant predictions. In Ref. [206], the $P_{c}(4380)$ and $P_{c}(4450)$ were interpreted as the loosely bound $\Sigma_{c} D^{*}$ and $\Sigma_{c}^{*} D^{*}$ molecules, respectively, in the one-boson-exchange model. In Ref. [207], the quantum numbers of these two states were suggested to be $J^{P}=3 / 2^{-}$and $5 / 2^{+}$, respectively. In Ref. [208], the masses of the pentaquarks with hidden beauty and strangeness were simply estimated with the heavy quark symmetry. For other studies of the hidden-charm pentaquark states before 2016, one may consult Refs. [1, 209]. Here we concentrate on recent developments with CMI models.

In Ref. [210], Maiani et al argued that the observed pentaquarks by LHCb are naturally expected diquark-diquark-antiquark states in an extended picture of Refs. [95, 129]. They assigned the compositions for the two $P_{c}$ states as

$$
\begin{aligned}
P\left(3 / 2^{-}\right) & =\left\{\bar{c}[c q]_{S=1}\left[q^{\prime} q^{\prime \prime}\right]_{S=1}, L=0\right\} \\
P\left(5 / 2^{+}\right) & =\left\{\bar{c}[c q]_{S=1}\left[q^{\prime} q^{\prime \prime}\right]_{S=0}, L=1\right\} .
\end{aligned}
$$

In Ref. [211], the pentaquarks were assumed as $\bar{c}[c u]_{\overline{3}_{c}}[u d]_{\overline{3}_{c}}$ diquark-diquark-antiquark states where all possible spins of diquarks were considered. Their masses were estimated with the formula taking into account the spin splitting,

$$
M_{P_{c}} \simeq m_{\bar{c}}+M_{[c u][u d]}=m_{\bar{c}}+m_{[c u]}+m_{[u d]}+J_{[c u][u d]}\left(J_{[c u][u d]}+1\right) \Delta
$$


where $M_{[c u][u d]}$ and $J_{[c u][u d]}$ are the mass and angular momentum of the diquark-diquark system. According to the obtained pentaquark masses in this analysis, the $P_{c}(4450)$ is a good candidate of $I\left(J^{P}\right)=1 / 2\left(5 / 2^{-}\right)$diquark-diquark-antidiquark state, but the $P_{c}(4380)$ was suggested to be a broad bump in the $3 / 2^{+}$-wave resulting from rescatterings in the $p J / \psi$ channel. This scheme differs from the classification in Refs. [210, 212]. In Ref. [213], the contributions from the possible $1^{+}[c n][\bar{c} \bar{s}]$ diquark-antidiquark states [133] with masses around $4189 \sim 4300 \mathrm{MeV}$ in the $K^{-} J / \psi$ channel of the $\Lambda_{b}^{0} \rightarrow K^{-} J / \psi p$ decay were discussed. The authors emphasised that such exotic mesons can imitate broad bumps in the $p J / \psi$ channel. The study of the pentaquark spectrum was extended to the strange $c \bar{c}$ case in Ref. [214]. Further discussions in this scheme can be found in Refs. [215, 216].

In Ref. [100], Zhu and Qiao studied the pentaquark states in the diquark-triquark picture where both diquark and triquark are not compact objects. Their investigated pentaquarks have the structure $\delta \bar{\theta}=[Q u]_{\overline{3}_{c}}[u d \bar{Q}]_{3_{c}}$ or $\delta \bar{\theta}=[Q u]_{\overline{3}_{c}}[u s \bar{Q}]_{3_{c}}(Q=c, b)$. Their masses were calculated with the model Hamiltonian (14), where the diquark masses were extracted from the $1^{++} X(3872)$ and the $1^{--} Y_{b}(10890)$ by assuming that they are diquark-antidiquark states, and the triquark masses were estimated by summing the quark masses. Their results suggest that the $P_{c}(4380)$ is a mixed state of three diquarktriquark structures with $J^{P}=3 / 2^{-}$, and the $P_{c}(4450)$ can be interpreted as the diquark-triquark pentaquark state $\left\{[c u]_{S=1}\left[(u d)_{S=0} \bar{c}\right]_{S=1 / 2}\right\}_{L=1}$ with $J^{P}=5 / 2^{+}$. The authors also predicted the spectra of $[c u][u s \bar{c}],[b u][u s \bar{b}]$, and $[b u][u s \bar{b}]$, and discussed possible production and decay processes for these pentaquark states.

In the $\bar{c} \mathcal{Q} \mathcal{Q}^{\prime}=\bar{c}[c q]\left[q^{\prime} q^{\prime \prime}\right](q=u, d, s)$ diquark-diquark-antiquark picture, Ali et al investigated the pentaquark spectrum using the extended model of (13) in Ref. [212],

$$
H=H_{\left[\mathcal{Q Q} \mathcal{Q}^{\prime}\right]}+H_{\bar{c}\left[\mathcal{Q Q} \mathcal{Q}^{\prime}\right]}+H_{S_{\mathcal{P}} L_{\mathcal{P}}}+H_{L_{\mathcal{P}} L_{\mathcal{P}}},
$$

where $L_{\mathcal{P}}$ and $S_{\mathcal{P}}$ are the orbital and spin angular momenta of the pentaquark state, respectively. They also adopted the extended model (12) by including additional spin-spin terms. Identifying the observed $P_{c}(4450)$ as the state $P\left(5 / 2^{+}\right)=\left\{\bar{c}[c u]_{S=1}[u d]_{S=0}, L=1\right\}$, the masses of the other $S$ - and $P$-wave pentaquark states were predicted. According to their results, identifying the state $P\left(3 / 2^{-}\right)=$ $\left\{\bar{c}[c u]_{S=1}[u d]_{S=1}, L=0\right\}$ to be the observed $P_{c}(4380)$ is problematic because its production from the $\Lambda_{b}^{0}$ decay is suppressed by the heavy quark symmetry. They suggest the LHCb Collaboration reanalyze their data to search for the lower $J^{P}=3 / 2^{-}$pentaquark in the range 4140-4130 MeV. In Ref. [217], the same authors extended their studies of the $J^{P}=3 / 2^{-}$and $J^{P}=5 / 2^{+}$pentaquark states to the $J^{P}=1 / 2^{ \pm}$cases. The anticipated discovery modes in $b$-baryons decays were also discussed.

In Ref. [108], we have systematically studied the $Q_{1} \bar{Q}_{2} q_{3} q_{4} q_{5}(Q=c, b, q=u, d, s)$ pentaquark states with the method (18), by assuming $Q_{1} \bar{Q}_{2}$ to be in the color 8 representation. The LHCb $P_{c}$ states fall in the mass region of the studied nnnc $\bar{c}$ system. Most such pentaquark states were found to have $S$-wave open-heavy decays and their widths should be broad. In contrast, the $J^{P}=5 / 2^{-}$states do not decay through the $S$-wave, so their widths should not be very broad. We also found low-mass $\Lambda$-type $u d s Q_{1} \bar{Q}_{2}$ states with $J^{P}=1 / 2^{-}$in this model. One may understand the existence of such a state from the CMI matrix elements. In the flavor $S U(3)$ symmetric limit, two states in the $1_{f}$ representation and three states in the $8_{f}$ representations have negative CMI matrix elements. Their mixing due to the symmetry breaking effectively provides additional attraction, which leads to a low-mass $\Lambda$-type pentaquark finally. This result agrees with the study in Ref. [218] where detailed investigations with one-gluon exchange and instanton-induced interactions were performed.

In the study of hidden-charm pentaquark states, Buccella discussed the masses of the compact $c \bar{c} u u d$ with the method similar to (18) in Ref. [219], where both $c c$ and uud are color octet states. For the $J^{P}=3 / 2^{-}$case, he predicted four pentaquarks around $4360 \mathrm{MeV}, 4409 \mathrm{MeV}, 4491 \mathrm{MeV}$, and 4560 $\mathrm{MeV}$. The first two states couple strongly to $J / \psi p$, and they appear as a resonance with a mass 4380 $\mathrm{MeV}$ in this channel, while the third and forth states couple strongly to $\Lambda_{c} \bar{D}^{*}$ and $\Sigma_{c} \bar{D}^{*}$, respectively. For the $J^{P}=5 / 2^{+} P_{c}(4450)$, the two color octets were assumed to be in $P$-wave. 
In Ref. [220], the authors assigned the observed $P_{c}(4380)$ as a compact uudce pentaquark with $J^{P}=3 / 2^{-}$in the lowest $S U_{f}(3)$ multiplet and considered its flavor-octet partner states using the extended Gürsey-Radicati (GR) formula [221],

$$
M_{G R}=M_{0}+A S(S+1)+D Y+E\left[I(I+1)-\frac{1}{4} Y^{2}\right]+G C_{2}(S U(3))+F N_{C}
$$

where $M_{0}$ is a scale parameter, $I$ is the isospin, $Y$ is the hypercharge, $C_{2}(S U(3))$ is the eigenvalue of the $S U_{f}(3)$ Casimir operator, and $N_{c}$ is a counter of $c$ or $\bar{c}$ quark. With the obtained parameters which were determined by fitting the masses of the ground state baryons, the authors found a state with the mass $4377 \mathrm{MeV}$ and the same quantum numbers as the $P_{c}(4380)$ (charge, spin, parity). Possible discovery channels in bottom baryon decays and the partial decay widths for all the pentaquarks were discussed. In Ref. [222], an extended $S U(4)$ quark model was developed. The mass formula for baryons has the form

$$
M^{2}=\left(\sum_{i} m_{i}\right)^{2}+M_{s f}^{2}+M_{o r b}^{2}
$$

where the spin-flavor operator $M_{s f}^{2}$ has a generalized form of the GR formula and the orbital contribution term has the form $M_{\text {orb }}^{2}=\alpha^{\prime} L$ with $\alpha^{\prime}$ being the linear trajectory slope. Applying the formula to the $J^{P}=3 / 2^{-} L=0 q q q c \bar{c}(q=u, d, s)$ states, the authors found that all the hidden-charm pentaquarks are below the relevant $J / \psi$-baryon thresholds. Therefore, the LHCb $P_{c}$ states were proposed to be the excited pentaquarks within this scheme.

\subsection{CMI and $Q Q q q \bar{q}$}

Up to now, the $\Xi_{c c}$ baryon is the unique one of the experimentally confirmed doubly heavy hadrons. Its observation motivated heated discussions on the possible $Q Q \bar{q} \bar{q}(Q=c, b, q=u, d, s)$ tetraquarks. If one adds one more light quark, the existence of the $Q Q q q \bar{q}$ pentaquark baryons might also be possible, because the light quark interactions inside the $q q \bar{q}$ cluster may provide stronger attractions and play an important role in binding the five quarks, especially when the color-spin mixing effects are considered. Compared with the conventional $Q Q q$ baryons, the $Q Q q q \bar{q}$ masses are probably not very large if the complicated interactions among quark components significantly lower their masses. As a result, the properties of the excited $Q Q q$ baryons may be affected by the $Q Q q q \bar{q}$ states through coupled channel effects. Knowledge about the basic features for the $Q Q q q \bar{q}$ spectra may be helpful for us to understand possible structures of heavy hadrons. In the literature, there exist some explorations of such systems which focus on the $(Q q q)-(Q \bar{q})$ molecules. However, such systems have not been paid enough attention to.

In Ref. [111], we have systematically estimated the masses of the possible $Q Q q q \bar{q}$ pentaquark masses in the CMI method (18). Our results suggest that there exist many states below relevant open-heavy baryon-meson thresholds, and thus stable pentaquarks are expected. Since the realistic pentaquarks probably have higher masses than the values in Ref. [111]. It is not very clear where the $(Q Q q)-(q \bar{q})$ type baryon-meson thresholds locate since most of the $(Q Q q)$ baryons have not been observed yet. Further study is needed to verify whether such states are stable or not. Our results can be a helpful guide for deeper investigations.

To search for stable pentaquark states, the authors of Ref. [223] presented a systematic analysis on the flavor-color-spin structures of the heavy quark pentaquark systems with the method (18). The authors noticed that the $I\left(J^{P}\right)=0\left(1 / 2^{-}\right) u d c c \bar{s}$ state is perhaps the most stable one. 


\subsection{CMI and $Q Q Q q \bar{q}$}

The $Q Q Q q \bar{q}$ system is the mirror-type structure of the $Q \bar{Q} q q q$ by exchanging the heavy and light flavors. Such states are related with the triply heavy conventional baryons, which have not been observed so far. This situation is similar to the relation between $Q \bar{Q}$ mesons and $Q \bar{Q} q \bar{q}$ tetraquark states. With the potential observations of $Q Q Q$ baryons in future measurements, it is also instructive to study the feature of the $Q Q Q q \bar{q}$ states. Besides, the study of the bound state and scattering problems induced by the observed $\Xi_{c c}$ baryons can help experiments to look for exotic phenomena.

In Ref. [112], we have discussed whether the compact $Q Q Q q \bar{q}$ pentaquark states are possible with the CMI method (18) by assuming that the $Q Q Q$ is always a color-octet state, similar to Ref. [108]. Our results indicate that the compact pentaquarks should not be stable against their rearrangement decays. On the other hand, the near-threshold baryon-meson molecules are possible, see Sec. 4. Therefore, the gap between the lowest threshold and the lowest compact pentaquark leaves room for the identification of hadronic molecules. If experiments could observe an exotic resonance around some low-lying threshold, its nature as a molecule will be preferred over a compact pentaquark. As a by-product, we conjectured the maximum values for the realistic masses of the conventional triply heavy baryons, which are consistent with most theoretical predictions in the literature.

\subsection{Mass constraints for $Q Q q$ and $Q Q Q$ baryons}

With the simplest CMI model (4), one cannot get accurate masses for conventional hadrons because of the adopted assumption, which has been illustrated in Sec. 2.3. The estimated multiquark masses with the model or its variants might also be far from the realistic values. Irrespective of the results, however, we find that some mass constraints for conventional hadrons $Q Q q$ and $Q Q Q$ may be conjectured when estimating multiquark masses with the CMI method (18).

In some cases, there may be two or more reference thresholds when one discusses a system with the help of Eq. (18). Obviously, they lead to different multiquark masses. Then one may establish an inequality for the thresholds of these reference systems. If one reference system contains a hadron without measured mass, the inequality can be used to constrain the mass of that hadron, e.g. $Q Q q$ or $Q Q Q$. For convenience, we use $H_{1}$ and $H_{2}\left(H_{1}^{\prime}\right.$ and $\left.H_{2}^{\prime}\right)$ to denote the two hadrons in the reference system $R\left(R^{\prime}\right)$. What we observed from the results for various systems is that the multiquark masses estimated with $R$ are usually higher than those with $R^{\prime}$, if both $H_{1}$ and $H_{2}$ contain heavy quarks while $H_{1}^{\prime}$ or $H_{2}^{\prime}$ does not contain heavy quarks, i.e.

$$
M_{H_{1}}+M_{H_{2}}-\left\langle H_{C M I}\right\rangle_{H_{1}}-\left\langle H_{C M I}\right\rangle_{H_{2}}>M_{H_{1}^{\prime}}+M_{H_{2}^{\prime}}-\left\langle H_{C M I}\right\rangle_{H_{1}^{\prime}}-\left\langle H_{C M I}\right\rangle_{H_{2}^{\prime}}
$$

If $H_{2}^{\prime}$ does not contain heavy quarks, that means

$$
M_{H_{1}^{\prime}}<\left[M_{H_{1}}-\left\langle H_{C M I}\right\rangle_{H_{1}}\right]+\left[M_{H_{2}}-\left\langle H_{C M I}\right\rangle_{H_{2}}\right]-\left[M_{H_{2}^{\prime}}-\left\langle H_{C M I}\right\rangle_{H_{2}^{\prime}}\right]+\left\langle H_{C M I}\right\rangle_{H_{1}^{\prime}} .
$$

For example, in estimating the masses of the $\operatorname{cccs} \bar{s}$ states, the reference systems may be $\Omega_{c c} D_{s}$ or $\Omega_{c c c} \phi$, and one expects that the latter system leads to lower pentaquark masses. Then we get the upper bound for the mass of $\Omega_{c c c}$ (see Table 3). If another system is considered, e.g. $\operatorname{cccs} \bar{n}$, a different value of mass can be obtained. The minimum mass should be the upper bound. In Ref. [112], we have obtained mass inequalities between the triply heavy baryons and the doubly heavy baryons with this feature, which are also collected in Table 3.

With the help of Ref. [109], we may similarly constrain the masses of doubly heavy baryons with $Q q q$ baryons and $Q \bar{q}$ mesons. Such inequalities are also shown in Table 3. Numerically, the mass within this approach is smaller than the upper limit with Eq. (4), which is illustrated by comparing masses in Tables 2 and 3. Up to now, only the doubly charmed baryon $\Xi_{c c}$ was discovered by LHCb and no other 
baryons containing two or more heavy quarks are observed. It is obvious that the measured mass of $\Xi_{c c}$ is consistent with the upper bound for $\Xi_{c c}$. For other baryon states, one may compare the constraints given here with the masses in the literature [104, 224, 225, 102, 112]. We find most of the theoretical predictions satisfy such constraints.

Since the masses in the second column of Table 3 are physical values, these constraints can be tested only after all the relevant ground-state baryons are observed. In this sense these inequalities are just conjectures. With these constraints, we may judge whether an observed heavy baryon is a ground state or not, i.e., once experiments observed states higher than such bounds, they should not be ground states.

Table 3: The conjectured constraints on the masses of ground-state triply and doubly heavy baryons, where the hadron symbols in the second column represent their masses. The estimations for the upper limits are given in units of $\mathrm{MeV}$. We have used the estimations for the upper limits of doubly heavy baryons when obtaining those for triple heavy baryons.

\begin{tabular}{ccc}
\hline \hline State & Upper limit & Estimation \\
\hline$\Omega_{c c c}$ & $\left(\Omega_{c c}^{*}+D_{s}-\phi\right)+\frac{16}{3}\left(C_{s \bar{s}}+3 C_{c \bar{s}}+C_{c c}-C_{c s}\right)$ & 4977 \\
$\Omega_{b b b}$ & $\left(\Omega_{b b}^{*}+B_{s}-\phi\right)+\frac{16}{3}\left(C_{s \bar{s}}+3 C_{b \bar{s}}+C_{b b}-C_{b s}\right)$ & 14937 \\
$\Omega_{b b c}^{*}$ & $\left(\Omega_{b b}^{*}+D_{s}-\phi\right)+\frac{16}{3}\left(C_{s \bar{s}}+3 C_{c \bar{s}}+C_{b c}-C_{b s}\right)$ & 11610 \\
$\Omega_{c c b}^{*}$ & $\left(\Omega_{b c}^{*}+D_{s}-\phi\right)+\frac{8}{3}\left(2 C_{s \bar{s}}+6 C_{c \bar{s}}+C_{c c}+C_{b c}-C_{c s}-C_{b s}\right)$ & 8285 \\
$\Omega_{b b c}$ & $\Omega_{b b c}^{*}-16 C_{b c}$ & 11577 \\
$\Omega_{c c b}$ & $\Omega_{c c b}^{*}-16 C_{b c}$ & 8253 \\
\hline$\Xi_{c c}$ & $\left(\Xi_{c}^{\prime}+D_{s}-\phi\right)+\frac{8}{3}\left(C_{c c}-C_{n s}-2 C_{c n}+2 C_{c s}+2 C_{s \bar{s}}+6 C_{c \bar{s}}\right)$ & 3669 \\
$\Xi_{b b}$ & $\left(\Xi_{b}^{\prime}+B_{s}-\phi\right)+\frac{8}{3}\left(C_{b b}-C_{n s}-2 C_{b n}+2 C_{b s}+2 C_{s \bar{s}}+6 C_{b \bar{s}}\right)$ & 10347 \\
$\Omega_{c c}$ & $\left(\Omega_{c}+D_{s}-\phi\right)+\frac{8}{3}\left(C_{c c}-C_{s s}+2 C_{s \bar{s}}+6 C_{c \bar{s}}\right)$ & 3799 \\
$\Omega_{b b}$ & $\left(\Omega_{b}+B_{s}-\phi\right)+\frac{8}{3}\left(C_{b b}-C_{s s}+2 C_{s \bar{s}}+6 C_{b \bar{s}}\right)$ & 10474 \\
$\Xi_{b c}^{*}$ & $\left.\Xi_{b}^{\prime}+D_{s}-\phi\right)+\frac{8}{3}\left(C_{b c}-C_{n s}+C_{c n}+3 C_{b n}+2 C_{b s}+2 C_{s \bar{s}}+6 C_{c \bar{s}}\right)$ & 7048 \\
$\Omega_{b c}^{*}$ & $\left(\Omega_{b}+D_{s}-\phi\right)+\frac{8}{3}\left(C_{b c}-C_{s s}+C_{c s}+5 C_{b s}+2 C_{s \bar{s}}+6 C_{c \bar{s}}\right)$ & 7174 \\
$\Xi_{c c}^{*}$ & $\Xi_{c c}+16 C_{c n}$ & 3733 \\
$\Xi_{b b}^{*}$ & $\Xi_{b b}+16 C_{b n}$ & 10368 \\
$\Omega_{c c}^{*}$ & $\Omega_{c c}+16 C_{c s}$ & 3871 \\
$\Omega_{b b}^{*}$ & $\Omega_{b b}+16 C_{b s}$ & 10493 \\
$\Xi_{b c}$ & $\Xi_{b c}^{*}-\frac{8}{3}\left(2 X_{n}+Y_{n}\right)$ & 6996 \\
$\Xi_{b c}^{\prime}$ & $\Xi_{b c}^{*}-\frac{8}{3}\left(2 X_{n}-Y_{n}\right)$ & 7022 \\
$\Omega_{b c}$ & $\Omega_{b c}^{*}-\frac{8}{3}\left(2 X_{s}+Y_{s}\right)$ & 7117 \\
$\Omega_{b c}^{\prime}$ & $\Omega_{b c}^{*}-\frac{8}{3}\left(2 X_{s}-Y_{s}\right)$ & \\
\hline \hline & &
\end{tabular}

\subsection{Effective CMI for multiquark states}

When studying the properties of the $Q Q \bar{Q} \bar{Q}$ tetraquark states [110], we checked the mass shifts due to variations of coupling parameters. What the mass shift reflects is the effective color-spin interaction between a pair of quark components. For a multiquark state, its mass $M$ in the CMI model can be 
expressed as a function of the effective quark masses and coupling constants

$$
M=M\left(m_{n}, m_{s}, m_{c}, m_{b}, C_{12}, C_{13}, \cdots\right) .
$$

In the case that mixing among different color-spin structures is considered, this mass function cannot be expressed as a simple formula like Eq. (9). However, we may approximately use a linear form to show the relation between $M$ and the parameters. To do that, we consider effective chromomagnetic interactions by making a change on the coupling constants. When one reduces a parameter $C_{i j}$, the mass of the state becomes larger or smaller. If the effective CMI between the $i$ th quark component and the $j$ th quark component is attractive (repulsive), the reduction of $C_{i j}$ will lead to a larger (smaller) $M$. This effect can be reflected by a dimensionless measure, which was defined in Ref. [112],

$$
K_{i j}=\frac{\Delta M}{\Delta C_{i j}}
$$

Here $\Delta C_{i j}$ is the variation of the coupling parameter $C_{i j}$, and $\Delta M=\Delta\left\langle H_{C M I}\right\rangle$ is the corresponding variation of the multiquark mass. When $\Delta C_{i j} \rightarrow 0, K_{i j} \rightarrow \frac{\partial M}{\partial C_{i j}}$ becomes a constant, then we have

$$
M=M_{0}+\sum_{i<j} K_{i j} C_{i j}
$$

where $M_{0}=\sum_{i=1} m_{i}$ of Eq. (4) or $M_{0}=M_{r e f}-\left\langle H_{C M I}\right\rangle_{r e f}$ of Eq. (18). The validity of this mass formula in the mixing case is easy to be checked with numerical results. Obviously, negative (positive) $K_{i j}$ indicates attractive (repulsive) effective CMI.

In practice, the value of $K_{i j}$ can be extracted by reducing $C_{i j}$ slightly, while it can also be derived with the matrix $\left\langle H_{C M I}\right\rangle$ and its eigenvalues. Consider a mixed state

$$
\psi_{\text {mix }}=x_{k} \varphi_{k}
$$

which is a mixture of different bases $\varphi_{k}$ 's with corresponding coefficients $x_{k}$ 's. If $E_{C M I}$ represents an eigenvalue of $H_{C M I}$, one has

$$
E_{C M I}=\left\langle\psi_{m i x}\left|H_{C M I}\right| \psi_{m i x}\right\rangle=x_{k} x_{l}\left\langle\varphi_{k}\left|H_{C M I}\right| \varphi_{l}\right\rangle
$$

The Hamiltonian acting on bases can be expressed as (see Eq. (11))

$$
\left\langle\varphi_{k}\left|H_{C M I}\right| \varphi_{l}\right\rangle=\left\langle H_{C M I}\right\rangle_{k l}=\sum_{m<n} X_{m n}^{k l} C_{m n}
$$

where $X_{m n}^{k l}$ can be read out from the obtained $\left\langle H_{C M I}\right\rangle$. Then

$$
E_{C M I}=x_{k} x_{l}\left\langle\varphi_{k}\left|H_{C M I}\right| \varphi_{l}\right\rangle=x_{k} x_{l}\left\langle H_{C M I}\right\rangle_{k l}=\sum_{m<n}\left(x_{k} X_{m n}^{k l} x_{l}\right) C_{m n}=\sum_{m<n} K_{m n} C_{m n}
$$

i.e., $K_{m n}=x_{k} X_{m n}^{k l} x_{l}$. With this expression, one can understand how contributions from different structures affect the multiquark mass.

Although Eq. (30) has a linear form, it does not mean that the multiquark mass is linearly related with the coupling parameters, because $K_{i j}$ 's also depend on $C_{i j}$ 's. The effects on the multiquark mass due to uncertainties of coupling parameters can only be seen with the values of $K_{i j}$ roughly. If the absolute value of a $K_{i j}$ is large, it is necessary to reduce the uncertainty of the corresponding $C_{i j}$ as much as possible. This possibility of large $K_{i j}$ can appear in some multiquark states. An application of the measure is to qualitatively guess the stability of tetraquark states. For states with the configuration 
$q_{1} q_{2} \bar{q}_{3} \bar{q}_{4}$, their dominant decays are through the fall-apart or rearrangement mechanism. However, there still exist some special configurations which lead to relatively stable states although their masses may be high. For example, if both the $q_{1} q_{2}$ and $q_{3} q_{4}$ interactions in a state are effectively attractive while other quark-antiquark interactions are repulsive, such a state should be relatively difficult to fall apart into meson-meson decay channels. From the systematic study of the $Q q \bar{Q} \bar{q}(Q=c, b, q=u, d, s)$ systems [113], the second highest $J^{P}=0^{+}$states usually satisfy this condition, which is helpful for us to identify the possible nature of the exotic mesons. However, it is not easy to qualitatively discuss the relative stability of pentaquark states with $K_{i j}$ 's.

\subsection{A short summary for CMI}

Table 4: All types of heavy quark tetraquark and pentaquark systems $(Q=c, b, q=u, d, s)$. The states which were discussed in literatures are given with bold fonts and the systems that we investigated and reviewed here are marked with a dagger.

\begin{tabular}{|c|c|}
\hline Tetraquarks & Pentaquarks \\
\hline$Q q \bar{q} \bar{q}$ & $\bar{Q} q q q q, Q q q q \bar{q}$ \\
\hline$Q \bar{Q} q \bar{q}^{\dagger}, Q Q \bar{q} \bar{q}^{\dagger}$ & $Q \bar{Q} q q q^{\dagger}, Q Q q q \bar{q}^{\dagger}$ \\
\hline$q Q \bar{Q} \bar{Q}^{\dagger}$ & $q \bar{q} Q Q Q^{\dagger}, q q Q Q \bar{Q}$ \\
\hline$Q Q \bar{Q} \bar{Q}^{\dagger}$ & $\bar{q} Q Q Q Q, q Q Q Q \bar{Q}$ \\
\hline & $Q Q Q Q \bar{Q}$ \\
\hline
\end{tabular}

The CMI models play an important role in understanding the multiquark systems. Although the Hamiltonian is simple, the models do catch the basic features of spectra since the mass splittings of hadrons reply on the basic controlling symmetries of the quark world. In recent years, developments about the CMI model investigations lead to series of important results. All types of heavy quark tetraquark and pentaquark systems that could exist in nature are listed in Table 4 . We have reviewed the CMI studies on four types of tetraquark systems and three types of pentaquark systems in this section. Some interesting observations are:

- The $Q \bar{Q} q \bar{q}$ states. The $X(4140), X(4274)$, and $X(4350)$ observed in $J / \psi \phi$, the $X(3860)$ observed in $D \bar{D}$, and the $Z_{c}(4100)$ observed in $\eta_{c} \pi$ can be consistently assigned as the compact tetraquark states with $J^{P C}=1^{++}, 1^{++}, 0^{++}, 0^{++}$, and $0^{++}$, respectively within the framework of the chromomagnetic interactions. Additional high-lying and relatively narrow tetraquark states are also expected whose effective chromomagnetic interactions inside the diquarks are attractive while those between quark and antiquarks are repulsive. Uncovering the inner structures of the exotic $X Y Z$ states needs more experimental investigations and further theoretical studies from various aspects.

- The $Q Q \bar{q} \bar{q}$ states. From the quark mass ratio $m_{q} / m_{Q}$ dependence of the $Q Q \bar{q} \bar{q}$ masses, the existence of such doubly heavy tetraquarks becomes inevitable when the ratio is small enough. From the CMI model calculations, it is still unclear whether the lowest $c c \bar{u} \bar{d}$ state is bound or not depending on the details of the quark interactions. An interesting possibility would be that the mass of the $T_{c c}$ is around the $D D^{*}$ threshold, similar to the masses of $X(3872)$ and $Z_{c}(3900)$. On the other hand, the existence of the bound $T_{b b}$ seems without doubt. The $T_{c c}$ and $T_{b b}$ states have only repulsive color-spin interactions inside the heavy diquarks. In contrast, not only the 
lowest but also heavier $b c \bar{u} \bar{d}$ states may exist with relatively narrow widths because the effective color-spin interaction inside the $b c$ and $u d$ diquarks can be both attractive. The existence of the doubly heavy tetraquarks is certainly one of the most important issues in hadron physics.

- The $Q Q \bar{Q} \bar{Q}$ and $Q Q \bar{Q} \bar{q}$ states. The lowest compact $c c \bar{c} \bar{c}$ and $b b \bar{b} \bar{b}$ states with $J^{P C}=0^{++}$from the CMI models seem unstable if all the color-spin structures are considered. Although the compact $b c \bar{b} \bar{c}$ and $b b \bar{b} \bar{c}$ states may also have rearrangement decay patterns, the effectively attractive colorspin interactions inside the diquarks probably lead to relatively narrow tetraquarks. Searching for $b c \bar{b} \bar{c}$ states in the $J / \psi \Upsilon$ channel seems to be feasible at LHC. Moreover, the relatively narrow $b c \bar{c} \bar{q}$ and $b c \bar{b} \bar{q}$ tetraquarks probably exist.

- The $Q \bar{Q} q q q$ states. Although the $P_{c}(4380)^{+}$and $P_{c}(4450)^{+}$states can be assigned as compact $c \bar{c} u u d$ pentaquark states in the CMI models, their inner structures still remain unresolved. An interesting observation is that a low mass isoscalar $c \bar{c} u d s$ pentaquark is possible which may be searched for in the $J / \psi \Lambda$ and $\eta_{c} \Lambda$ channels. Similarly, the isoscalar $b \bar{c} u d s, c \bar{b} u d s$, and $b \bar{b} u d s$ states may be searched for in their rearrangement strong decay channels.

- The $Q Q q q \bar{q}$ and $Q Q Q q \bar{q}$ states. All the $\Xi_{c c}, T_{c c}$, and $c c q q \bar{q}$ (denoted as $P_{c c}$ ) states contain the $c c$ diquark. If the $T_{c c}$ were bound, the lowest $Q Q q q \bar{q}$ pentaquarks might also be bound states. Since the other $Q Q q$ baryons except $\Xi_{c c}$ have not been observed, it is not clear whether low mass structures such as isoscalar bcns $\bar{n}$, bcnn $\bar{s}$, and bcnn $\bar{n}$ have rearrangement decay channels or not yet. The isoscalar $c c n n \bar{n}, c c n n \bar{s}$, and $c c n s \bar{n}$ states are probably the most stable doubly charmed pentaquark. The triply heavy $Q Q Q q \bar{q}\left(P_{Q Q Q}\right)$ structures seem unstable.

\section{Constituent quark models}

Various versions of nonrelativistic and relativistic constituent quark models can be found in the literature, which were proposed to understand hadron properties. Almost all of them incorporate both the short-range one-gluon-exchange (OGE) force and the term representing the color confinement, in either the coordinate or momentum space. Some of them include the additional flavor-dependent Goldstone-boson-exchange (GBE) force from the spontaneously broken chiral symmetry and/or the screening effects from the quark-antiquark pair creation. There are also models containing only GBE and confinement potentials. In some approaches, one has to adopt the diquark and triquark approximations in order to reduce the few-body problem to a two-body problem. In this section, we focus on recent studies of compact multiquark states within constituent quark models. We will also discuss some $X Y Z$ states which are candidates of the conventional or hybrid charmonium.

One of the widely adopted potentials was proposed by Godfrey and Isgur [33, 34], which was very successful in explaining conventional meson and baryon spectra. Now we denote it as the GI model. In studying multiquark states, there also exist many types of potentials, two of which were very popular. They were proposed by Bhaduri, Cohler, and Nogami in Ref. [226], and by Semay and Silvestre-Brac in Refs. [149, 227]. The latter references provided four potentials corresponding to different fitted parameters, which we shall call as BCN and Grenoble (AL1, AP1, AL2, and AP2) in the present review. The potential between $i$ th and $j$ th quark components in the BCN model reads

$$
V_{i j}(r)=-\frac{3}{16} \vec{\lambda}_{i} \cdot \vec{\lambda}_{j}\left[\frac{\hbar^{2} \kappa_{\sigma}}{m_{i} m_{j} c^{2} r_{0}^{2}} \frac{e^{-r / r_{0}}}{r} \vec{\sigma}_{i} \cdot \vec{\sigma}_{j}-\frac{\kappa}{r}+\frac{r}{a^{2}}-\Delta\right],
$$

while that in the Grenoble model has the form

$$
V_{i j}=-\frac{3}{16} \vec{\lambda}_{i} \cdot \vec{\lambda}_{j}\left[\frac{2 \pi \kappa^{\prime}}{3 m_{i} m_{j}} \frac{\exp \left(-r^{2} / r_{0}^{2}\right)}{\pi^{3 / 2} r_{0}^{3}} \vec{\sigma}_{i} \cdot \vec{\sigma}_{j}-\frac{\kappa}{r}+\lambda r^{p}-\Lambda\right]
$$


with $r_{0}\left(m_{i}, m_{j}\right)=A\left(2 m_{i} m_{j} /\left(m_{i}+m_{j}\right)\right)^{-B}$.

In the following subsections, we shall separately review the theoretical progress of various constituent quark models on the $Q \bar{Q} q \bar{q}, Q Q \bar{q} \bar{q}, Q Q \bar{Q} \bar{Q}, Q Q \bar{Q} \bar{q}$, and $Q \bar{Q} q q q$ multiquark states.

\section{$3.1 Q \bar{Q} q \bar{q}$}

Some neutral $X Y Z$ states are probably the conventional quarkonium. In some cases, the bare quarkonium states are strongly affected by the couple-channel effects. A typical example is the $X(3872)$ [228] which is affected strongly by the $D \bar{D}^{*}$ scattering states. In fact, the mixing between the $Q \bar{Q}$ and $Q \bar{Q} q \bar{q}$ may be accounted for through the ${ }^{3} P_{0}$ quark pair creation in the vacuum, which could be equivalently incorporated by the screened potential. The $Q \bar{Q}$ core contributions could be calculated even at the hadron level through Weinberg's compositeness theorem [229]. On the other hand, the $Z$ states do not have a quarkonium core.

\subsubsection{Possible quarkonium or hybrid assignments}

The authors of Ref. [230] studied the charmonium spectrum and electromagnetic transitions between charmonium states in a nonrelativistic quark potential model where both the linear and screened confinement potentials are considered. Their results indicate that the possibility of the $X(3872)$ being a $\chi_{c 1}(2 P)$ dominant state cannot be excluded from its radiative decay properties. See also discussions in Ref. [231]. The $X(3823)$ as the $\psi_{2}(1 D)$ is supported by its radiative decay properties. The mass splitting between the $2^{3} P_{2}$ state and the $2^{3} P_{1}$ state does not support the assignment of the $X(3915)$ as the $\chi_{c 0}(2 P)$ state. In the screened potential model, the $Y(4260)$ and $Y(4360)$ may be good candidates of the $\psi(4 S)$ and $\psi_{1}(3 D)$, respectively. The $X(4140)$ or $X(4274)$ might be identified as the $\chi_{c 1}(3 P)$ state. From the subsequent study on the open-charm strong decays of the higher charmonium states in Ref. [232], the authors found that it is possible to assign the $Y(4660), X(4500), X(3940)$, and $X(3860)$ as $\psi(5 S), \chi_{c 0}(4 P), \eta_{c}(3 S)$, and $\chi_{c 0}(2 P)$, respectively. The assignment for the $X(4140)$ and $X(4274)$ in the same model as the excited $\chi_{c 1}$ states is difficult. The adopted model does not lead to a self-consistent description for the vector $Y(4230 / 4260,4360)$ and the scalar $X(4700)$.

In Ref. [233], the authors investigated possible assignments for the four $J / \psi \phi$ structures in a coupled channel scheme by using a nonrelativistic constituent quark model [234, 235]. They found that the $X(4140)$ seems to be a cusp because of the near coincidence of the $D_{s} D_{s}^{*}$ and $J / \psi \phi$ thresholds, while the $X(4274), X(4500)$, and $X(4700)$ appear as conventional $3^{3} P_{1}, 4^{3} P_{0}$, and $5^{3} P_{0}$ charmonia, respectively.

In Ref. [236], a relativistic Dirac potential model inspired by the OGE interaction was developed to study the charmonium spectrum in the momentum space. Due to the relativistic nature of the model, the spin-orbit, spin-spin, and tensor effects were all automatically included in the calculation. Two types of the scalar potentials with screening factors were considered. The authors determined the model parameters by fitting the masses of the eight resonances below the $D \bar{D}$ threshold and reproduced the overall structure of the charmonium spectrum. Analysing the predicted masses for high-lying resonances, they identified the $X(3915), X(3872)$, and $\chi_{c 2}(2 P)$ as $2^{3} P_{J}$ states with $J=0,1$, and 2, respectively, while the $X(3915)$ is not well described and the $X(3872)$ is slightly higher than the measured mass. The $\psi(3823)$ was well reproduced, a result consistent with Ref. [230], but it is difficult to accommodate the $Y(4260), Y(4360), Y(4660), X(3940), X(4160)$, and $X(4140)$ from their results. The authors also suggested to perform deeper dynamical studies by considering effects such as molecule/tetraquark admixture and threshold effects.

In studying the properties of the exotic states, the authors of Ref. [237] discussed their possible heavy quarkonium assignments by solving the relativistic Dirac equation with a linear confinement potential. Their results indicate that the $X(4140)$ is a mixture of two $P$-wave charmonia, and the 
$Y(4630)$ and $Y(4660)$ are admixed states of the $S-D$ waves. In Ref. [238], the spectroscopy of the charmonium states was investigated with a Coulomb plus linear potential. From their calculations, the authors assigned the $Y(4660), X(3872), X(3915)$, and $X(4274)$ to be the $5^{3} S_{1}, 2^{3} P_{1}, 2^{3} P_{0}$, and $3^{3} P_{1}$, respectively.

In Ref. [239], the masses and decay widths of the vector charmonium states from $J / \psi$ to $\psi(4160)$ were calculated by using the instantaneous Bethe-Salpeter equation with a screened Cornell potential and the ${ }^{3} P_{0}$ model by considering the mixing effects between the $(n+1) S$ and $n D$ states. Most of the results were consistent with experimental data. The authors further investigated the mixing between $4 S$ and $3 D$ states, and found that it is still possible to assign the $Y(4260)$ and $Y(4360)$ to be the $4 S-3 D$ mixed charmonium states. The branching ratios of their decays into the $D \bar{D}$ were predicted to be small.

In a study of the heavy quarkonium hybrids [240] based on the strong coupling regime of pNRQCD (potential nonrelativistic QCD), the authors found that most of the isospin zero $X Y Z$ states fit well either as the hybrid or standard quarkonium candidates. The $X(3823)$ is compatible with the charmonium $\psi_{2}(1 D)$. The $X(3872)$ is compatible with the $\chi_{c 1}(2 P)$, but mixing with $D^{0} \bar{D}^{0 *}$ may have large contributions. The $X(3915)$ and $X(3940)$ are compatible with the charmonium states $\chi_{c 0}(2 P)$ and $h_{c}(2 P)$, respectively, but $D_{s}^{+} D_{s}^{-}$may contribute here. The $Y(4008)$ is compatible with a $1^{--}$hybrid state, which mixes with a spin-1 charmonium. The $X(4140)$ and $X(4160)$ are compatible with the $1^{++}$hybrid states. These states may be affected by the $D_{s}^{*} \bar{D}_{s}$ threshold. The $Y(4230)$ and $Y(4260)$ are compatible with the $1^{--} 2 D$ charmonium state having a dominant spin- 0 hybrid component. The $X(4274)$ is compatible with the charmonium $\chi_{c 1}(3 P)$, which may be affected by the $D_{s}^{*+} D_{s}^{*-}$ threshold. The $X(4350)$ is a hybrid or a conventional $3 P$ charmonium. The $Y(4320), Y(4360)$, and $Y(4390)$ are compatible with the spin-0 $1^{--}$hybrid. The $X(4500)$ is compatible with a $0^{++}$hybrid state, but its mixing with the spin-1 charmonium is little and it is difficult to understand its observation in the $J \psi \phi$ channel. The $Y(4630)$ is compatible with the charmonium $\psi(3 D)$. The $Y(4660)$ is compatible with a spin-0 $1^{--}$hybrid state. The $X(4700)$ is compatible with the charmonium $\chi_{c 0}(4 P)$. In the bottom case, the $\Upsilon(10860)$ is compatible with the bottomonium $\Upsilon(5 S)$, the $Y_{b}(10890)$ [241] is compatible with the spin-0 $1^{--}$hybrid, and the $\Upsilon(11020)$ with the bottomonium $\psi(4 D)$.

In Ref. [242], a nonrelativistic effective field theory describing heavy quarkonium hybrids was constructed. The authors discussed possible hybrid assignments for the exotic $X$ and $Y$ states, such as the $Y(4220), X(4350)$, and $Y(4260)$. In Ref. [243], Miyamoto and Yasui studied the spectra and decay widths of the hybrid quarkonia in a hyperspherical coordinate approach, and discussed the possibility of the $Y(4260), Y(4360), \psi(4415), Y(4660)$, and $\Upsilon(10860)$ being the hybrid states. According to their results, in the charmonium sector, the ground state of a magnetic gluon hybrid and the first excited state of an electric gluon hybrid lie close to the $\psi(4415)$. In the bottomonium sector, the first excited electric hybrid and the ground magnetic hybrid appear a few hundred MeV above the $\Upsilon(10860)$. If one of the exotic meson candidates is a hybrid, its constituent gluon was expected to be magnetic. In a nonrelativistic quark model with the Cornell like potential, the authors of Ref. [244] found that assigning the $\Upsilon(10860)$ as a mixture of the conventional $\Upsilon(5 S)$ and the lowest hybrid state may give a plausible explanation for its $\pi \pi \Upsilon(n S)(n=1,2,3)$ production rates.

\subsubsection{Schemes of the $Q \bar{Q}$ core admixed with coupled channels}

Very recently, BESIII observed a new decay mode for the $X(3872), \pi^{0} \chi_{c 1}$, in the processes $e^{+} e^{-} \rightarrow$ $\gamma \pi^{0} \chi_{c J}(J=0,1,2)$ and found $\operatorname{Br}\left(X(3872) \rightarrow \pi^{0} \chi_{c 1}\right) / \operatorname{Br}\left(X(3872) \rightarrow \pi^{+} \pi^{-} J / \psi\right)=0.88_{-0.27}^{+0.33} \pm 0.10$ [245]. This new measurement disfavors the $\chi_{c 1}(2 P)$ interpretation for the $X(3872)$ presented in Ref. [246]. An early measured ratio $\operatorname{Br}(X(3872) \rightarrow \psi(2 S) \gamma) / \operatorname{Br}(X(3872) \rightarrow J / \psi \gamma)=3.4 \pm 1.4$ by BaBar [247] did not support a pure $\bar{D}^{0} D^{*}$ molecule interpretation, while Belle's result of $\operatorname{Br}(X(3872) \rightarrow$ $\psi(2 S) \gamma) / B r(X(3872) \rightarrow J / \psi \gamma)<2.1$ [248] indicates that the $c \bar{c}$ contribution in $X(3872)$ is probably not large. These measurements strongly indicate that the $X(3872)$ probably contains a larger $D \bar{D}^{*}$ 
component than the $c \bar{c}$. Inspired by these experimental measurements, there has been continuous progress of theoretical studies on the coupled nature of the $X(3872)$ and other $X$ and $Y$ states in recent years.

In Ref. [249], the authors investigated the quarkonium contributions to the meson molecules like the $X(3872)$ and its heavy-quark spin-flavor partners. They found that the $c \bar{c}$ core produces an extra attraction, and thus less attractive $D \bar{D}^{*}$ interaction is needed to form a bound $X(3872)$. But such an attraction does not appear in the $2^{++}$sector. The $c \bar{c}$ content in the $X(3872)$ was found to be $10 \sim 30 \%$.

With the extended Friedrichs scheme, Zhou and Xiao of Ref. [250] studied the radially excited $P$-wave charmonium spectrum. According to their analyses, the $X(3872)$ is dynamically generated by the coupling of the bare $\chi_{c 1}(2 P)$ and continuum states, with large molecule components $(64-85 \%)$. The observed $X(3860)$ has a much larger width than the $\chi_{c 0}(2 P)$ charmonium although their masses are close. The state $h_{c}(2 P)$ was predicted to be around $3890 \mathrm{MeV}$ with a pole width about $44 \mathrm{MeV}$. Later in Ref. [251], they considered the isospin breaking effects of the $X(3872)$ with the same scheme, and obtained the reasonable ratio $0.58 \sim 0.92$ between the $J / \psi \pi \pi \pi$ and $J / \psi \pi \pi$ channels. In Ref. [252], the bottomonium counterpart of the $X(3872)$ around $10615 \mathrm{MeV}$ and the $\chi_{b 1}(4 P)$ around $10771 \mathrm{MeV}$ were predicted. The reason for the non-observation of the $X_{b}$ signal in the $\Upsilon \pi \pi$ [253] and $\Upsilon \pi \pi \pi$ [254] channels was also discussed.

In Ref. [255], the coupled channel effects on the heavy quarkonium states like $\chi_{\mathrm{c}}(2 P)$ and $\chi_{\mathrm{b}}(3 P)$ were studied by considering the meson loop corrections. The authors found that the $X(3872)$ is a superposition of a $c \bar{c}$ core and meson-meson continuum components, while the $\chi_{b}(3 P)$ should be a pure bottomonium. The authors of Ref. [256] presented an unquenched method to consider the coupling between the quark-antiquark states and meson-meson states for a given $J^{P C}$. After the investigation on channels related with the $X(3872)$, the authors got three states. The state that can be realized as the $X(3872)$ has a dominant $D \bar{D}^{*}$ component and a posterior $2^{3} P_{1} c \bar{c}$. The state identified as the $X(3940)$ has a predominant $2^{3} P_{1} c \bar{c}$ component. The third state is an almost pure $1^{3} P_{1} c \bar{c}$ state. The coupled channel effects for the bottomonium with realistic wave were also studied under the framework of ${ }^{3} P_{0}$ model in Ref. [257].

By assuming the $X(3872)$ as a mixing state of the $2 \mathrm{P}$ charmonium and $\bar{D} D^{*}$ molecule, the authors of Ref. [258] analyzed its radiative decays into $J / \psi \gamma$ and $\psi(2 S) \gamma$ in an effective field theory. They found that a wide range of charmonium probability in the $X(3872)$ is consistent with the experimental ratio $\operatorname{Br}(X \rightarrow \psi(2 S) \gamma) / B r(X \rightarrow J \psi \gamma)$. In the case of the destructive interferences between the long-range meson loops and the short-range counter-term, a strong constraint on the $c \bar{c}$ admixture is found $(<15 \%$ is expected).

To understand the nature of $X(3872)$, the spectral function of the charmonium state $\chi_{c 1}(2 P)$ coupled to $D D^{*}$ mesons was studied in Ref. [259]. The authors found two poles in the complex plane, one pole corresponding to the $c \bar{c}$ object $\chi_{c 1}(2 P)$ and the other virtual pole just below the $D^{0} D^{* 0}$ threshold. The $X(3872)$ as a mixed object emerges from an interplay between a $c \bar{c}$ state and the thresholds. With their approach, the existence of $X(3872)$ is not possible without the seed charmonium state.

In Ref. [260], the $Y(4260)$ was treated as an $S$-wave $\bar{D} D_{1}+$ c.c. molecule containing a small charmonium component. Extracting parameters from experimental results, the authors found that the physical wave function of the $Y(4260)$ is $0.363|c \bar{c}\rangle+0.932 \mid \bar{D} D_{1}+$ c.c. $\rangle$. In Ref. [261], Lu, Anwar, and Zou studied the coupling between the $1^{--}$charmonium structures and relevant meson-antimeson structures with the ${ }^{3} P_{0}$ pair creation model. Their results support the picture that the $Y(4260)$ is a $D_{1} \bar{D}$ molecule having a non-negligible $\psi(n D)$ core.

\subsubsection{Tetraquark states}

In the chiral $\mathrm{SU}(3)$ quark model [262], the masses of the $Q q \bar{Q} \bar{q}^{\prime}(Q=c, b, q=u, d, s)$ states with $J^{P C}=(0,1,2)^{++}$and $1^{+-}$were calculated. It was impossible to assign the $X(3872)$ and $Y(3940)$ 
[now called $X(3915)$ ] as pure $c n \bar{c} \bar{n}$ tetraquark states with $J^{P C}=1^{++}$and $2^{++}$, respectively. From this study, narrow tetraquarks in the hidden-bottom sector are possible. The study of the spectrum in the framework of the constituent quark model in Ref. [263] did not show any bound four-quark $c \bar{c} n \bar{n}$ states for low-lying $J^{P C}$, ruling out the possibility that the $X(3872)$ is a compact tetraquark system, if no additional correlations are considered in simple quark models.

In Ref. [264], the $Z_{c}(3900), Z_{c}(4020), Z_{c}(4050), Z_{b}(10610)$, and $Z_{b}(10650)$ were studied within a coupled-channel Schrödinger model by coupling the $I^{G}\left(J^{P C}\right)=1^{-}\left(1^{++}\right)$and $1^{+}\left(1^{+-}\right)$excited quarkantiquark pairs and their OZI-allowed decay channels $D^{(*)} \bar{D}^{(*)}$ or $B^{(*)} \bar{B}^{(*)}$. Poles matching experimental data for these mesons were all found. In Ref. [265], the nature of the $\Upsilon(10890)$ and other exotic states in the bottom sector was discussed in the diquark-antidiquark configuration in a quark potential model. The authors found that the $Z_{b}(10650)$ is probably a radially excited diquark-antidiquark state, and that the $\Upsilon(10890)$ might be the tetraquark $Y_{b}(10890)$ rather than a conventional $b \bar{b}$ state.

In Ref. [266], Lu and Dong presented a study for the $[c s]_{\overline{3}_{c}}[\bar{c} \bar{s}]_{3_{c}}$ diquark-antidiquark states in the GI model by including the color screening effects. In this model, the $X(4140)$ can be regarded as the $A \bar{S}$ type tetraquark state, and the $X(4700)$ as the first radially excited $A \bar{A}$ or $S \bar{S}$ state, where $S(A)$ denotes the scalar (axial-vector) diquark. They also studied the case that the internal orbital or radial excitation is allowed for diquarks $[267,268]$. Their results indicate that the $X(4500)$ can be explained as the tetraquark composed of one $2^{1} S_{0}$ diquark and one $1^{1} S_{0}$ antidiquark, while the $X(4350)$ is a tetraquark composed of one $2^{3} S_{1}$ and one $1^{3} S_{1}$. However, there is no assignment for the $X(4274)$ in this model, which is proposed to be the candidate of the charmonium $\chi_{c 1}(3 P)$, supported by the decay width calculation in Ref. [269] and the arguments in Ref. [270]. Their results also indicate that it is possible to assign the $X(3915)$ as the lightest $c s \bar{c} \bar{s}$ state, which supports the proposal given in Ref. [135]. Besides, some tetraquark masses are close to those of the $Y(4630)$ and $Y(4660)$.

In Ref. [271], possible $D^{(*)} \bar{D}^{*}$ molecular states were studied in the BCN model and a chiral constituent quark model by including the $s$-channel one gluon exchange. The authors found the $J^{P C}=(1,2)^{++}$bound states in the charm sector and $(0,1,2)^{++}, 1^{+-}$bound states in the bottom sector. Good candidates for the $X(3872)$ and $Z_{b}(10610)$ were then obtained. In another work [272], the same authors considered the $S$-wave $\bar{Q} Q \bar{q} q(Q=c, b, q=u, d, s)$ systems with two chiral constituent quark models in the meson-antimeson picture. From their results, several $B \bar{B}^{*}$ bound states were found. If the hidden-color channels are also included, a bound state $[c \bar{q}]^{*}[q \bar{c}]^{*}$ with $I\left(J^{P C}\right)=1\left(0^{++}\right)$was also found. The obtained $B \bar{B}^{*}$ and $B^{*} \bar{B}^{*}$ states with $I\left(J^{P C}\right)=1\left(1^{+-}\right)$can be related to the $Z_{b}(10610)$ and $Z_{b}(10650)$, respectively.

In Ref. [273], the investigation for the spectroscopy of the hidden-charm $[q c]_{\overline{3}_{c}}[\bar{q} \bar{c}]_{3_{c}}$ and $[s c]_{\overline{3}_{c}}[\bar{s} \bar{c}]_{3_{c}}$ tetraquarks was performed in a relativized diquark model. The authors found possible assignments for the $X(3872), Z_{c}(3900), Z_{c}(4020), Y(4008), Z_{c}(4240), Y(4260), Y(4360), Y(4630)$, and $Y(4660)$ in the $c \bar{c} n \bar{n}$ sector, and for the $X(4140), X(4500)$, and $X(4700)$ in the $c \bar{c} s \bar{s}$ sector, but did not for the $X(4274)$ and $Z_{c}(4430)$.

In the dynamical diquark model, the authors of Ref. [274] presented a study on the spectrum of charmoniumlike tetraquarks by solving the Schrödinger equation with the Born-Oppenheimer potentials calculated numerically on the lattice. Choosing the $X(3872)$ or $Z_{c}(4430)$ as a reference diquarkantidiquark state, the authors obtained a spectrum that agrees well with the observed charmoniumlike states.

Yang and Ping adopted a chiral quark model with the exchange of $\pi, \kappa$, and $\eta$ and considered the diquark-antidiquark and meson-meson configurations in Ref. [275]. They investigated the spectrum of $c s \bar{c} \bar{s}$ states and found that the $X(4274)$ and $X(4350)$ are good candidates of the compact tetraquark states with $J^{P C}=1^{++}$and $0^{++}$, respectively. The $X(4700)$ can be explained as the $2 S$ radial excited tetraquark state with $J^{P C}=0^{++}$. However, no appropriate matching states for $X(4140)$ and $X(4500)$ were found. 


\section{$3.2 Q Q \bar{q} \bar{q}$}

The doubly heavy $Q Q \bar{q} \bar{q}$ tetraquark systems are particularly interesting depending on the spatial configurations of two heavy quarks. In the extreme case that the $Q Q$ pair is in the color anti-triplet and stays very close to each other, the compact heavy quark pair acts like one static color source. Two light anti-quarks circle around this point-like color source. This configuration is very similar to the Helium atom in QED. We denote it as the "QCD Helium atom". On the other hand, if the heavy quarks are well separated from each other, the $\bar{q} \bar{q}$ pair is shared by the two heavy quarks. This is the QCD valence bond. Such a configuration is the QCD analogue of the hydrogen molecule in QED. We denote it as the "QCD Hydrogen molecule". In general, the $Q Q \bar{q} \bar{q}$ tetraquark system may be the superposition of the atomic and molecular structures.

In Ref. [276], using the BCN potential and the Grenoble AL1 potential, the authors studied the binding problem of the $T_{c c}$ (the lowest isoscalar $1^{+} c c \bar{u} \bar{d}$, see Sec. 2.5). According to their calculation, the $T_{c c}$ with the molecular structure is weakly bound against the $D D^{*}$ threshold, but it can become atomic with the inclusion of the three-body force.

In Ref. [163], the masses of the tetraquark states $Q Q \bar{q} \bar{q}(Q=c, b, q=u, d, s)$ were calculated in the diquark-antidiquark picture with a relativistic quark model. All the $(c c)\left(\bar{q} \bar{q}^{\prime}\right)$ states are not bound, and only the $I\left(J^{P}\right)=0\left(1^{+}\right)(b b)(\bar{n} \bar{n})$ state lies below the $B B^{*}$ threshold. The authors of Ref. [277] studied the $Q Q \bar{q} \bar{q}$ four-quark bound states in chiral $S U(3)$ quark model. Their calculation indicates that a $b b \bar{n} \bar{n}$ state with $I\left(J^{P}\right)=0\left(1^{+}\right)$is bound, but there is no bound state in the $c c \bar{q} \bar{q}$ system. With three different quark models one of which is chiral quark model, the authors of Ref. [278] studied the $Q Q \bar{n} \bar{n}(Q=s, c, b, n=u, d)$ spectrum. Their results show that only the $b b \bar{n} \bar{n}$ state with $I\left(J^{P}\right)=0\left(1^{+}\right)$ is bound within these models.

The authors of Ref. [279] studied possible compact four-quark states $Q Q \bar{n} \bar{n}$ with two different constituent quark models, one of which contains Goldstone boson exchanges between quarks. They noticed that the $I\left(J^{P}\right)=0\left(1^{+}\right) c c \bar{n} \bar{n}$ and $b b \bar{n} \bar{n}$ are bound and should be narrow, which was consistent with their previous studies [280, 281, 282]. Further study of the exotic $b c \bar{n} \bar{n}$ four-quark states in Ref. [283] showed two isoscalar bound states with $J^{P}=0^{+}$and $1^{+}$. In Ref. [284], the Bethe-Salpeter equations for the ground-state $Q Q \bar{u} \bar{d}$ were established in the diquark-antidiquark picture. According to their numerical results, the $c c \bar{u} \bar{d}$ and $b b \bar{u} \bar{d}$ bound states with $I\left(J^{P}\right)=0\left(1^{+}\right)$and the $b c \bar{u} \bar{d}$ bound states with $I\left(J^{P}\right)=0\left(0^{+}\right)$and $0\left(1^{+}\right)$should all be stable.

In Ref. [285], Park and Lee investigated the $1^{+} T_{b b}$ and $T_{c c}$ states with the BCN potential by including the contribution from the $6_{c}-\overline{6}_{c}$ diquark-antidiquark color structure, which was neglected in Ref. [152]. The $T_{c c}$ was found to be $100 \mathrm{MeV}$ above the $D D^{*}$ threshold, while the $T_{b b}$ was about 100 $\mathrm{MeV}$ below the $B B^{*}$ threshold. The $6_{c}-\overline{6}_{c}$ contribution was found to be negligible. Later in Ref. [286], the masses of the doubly heavy tetraquarks were updated after the observation of the $\Xi_{c c}$. Now the $u d \bar{b} \bar{b}$ tetraquark is bound by $121 \mathrm{MeV}$ and the $u s \bar{b} \bar{b}$ is bound by $7 \mathrm{MeV}$. In an investigation with one gluon exchange potential, the authors of Ref. [287] studied the existence of the bound $Q_{1} \bar{q}_{2} Q_{3} \bar{q}_{4}$ states. They found that there are no stable $c c \bar{n} \bar{n}$ and $b c \bar{n} \bar{n}$ states, but stable $b b \bar{u} \bar{d}$ states are possible.

The authors of Ref. [288] discussed approximations used in the multiquark studies, i.e., diquark, Born-Oppenheimer, Hall-Post inequalities, color-mixing, and spin-dependent corrections. They pointed out that the $1^{+} c c \bar{u} \bar{d}$ is at the edge of binding, and more delicate studies are still needed. For the $1^{+}$ $b b \bar{u} \bar{d}$, the spin effects or color mixing effects are needed to achieve a binding state.

\section{3 $Q Q \bar{Q} \bar{Q}$ and $Q Q \bar{Q} \bar{q}$}

The study in Ref. [289] with a nonrelativistic potential model indicates that the lowest $0^{++}$all-charm tetraquark state is below the $\eta_{c} \eta_{c}$ threshold. A study for the fully-heavy tetraquarks was performed in Ref. [290] with a nonrelativistic effective field theory (NREFT) at the leading order and a relativized 
diquark-antidiquark model. Both approaches give a $b b \bar{b} \bar{b}$ ground state around $18.72 \mathrm{GeV}$ with $J^{P C}=$ $0^{++}$, which is below the $\eta_{b} \eta_{b}$ threshold. Mass inequalities for all type $Q Q \bar{Q} \bar{Q}(Q=c, b)$ were also investigated.

The authors of Ref. [291] presented a calculation for the mass spectrum of the ground-state $0^{++}$ $b b \bar{b} \bar{b}$ tetraquark states using a diffusion Monte-Carlo method to solve the non-relativistic many-body problem, whose potential is based on the flux-tube model. A state around $18.69 \mathrm{GeV}$ was found to be about $100 \mathrm{MeV}$ below the $\eta_{b} \eta_{b}$ threshold.

In Ref. [292], the masses of the $c c \bar{c} \bar{c}$ tetraquark states were calculated in a constituent quark model with the hyperspherical formalism. The authors noted that the $0^{++}$state is $58 \mathrm{MeV}$ above the $\eta_{c} \eta_{c}$ threshold, $1^{+-}$state is $14 \mathrm{MeV}$ above the $J / \psi \eta_{c}$ threshold, and $2^{++}$state is $22 \mathrm{MeV}$ below the $J / \psi J / \psi$ threshold. In Ref. [293], the authors investigated the spectroscopy of $[c c][\bar{c} \bar{c}]$ in a diquark-antidiquark configuration using a non-relativistic model, whose potential is a Cornell-inspired type. Their results show very compact tetraquarks, which are below their thresholds of spontaneous dissociation into lowlying charmonium pairs.

In Ref. [294], Richard et al found that full-charm and full-beauty tetraquarks are unbound in the chromoelectric model with additive potentials. If the naive color-additive model of confinement is replaced by a string-inspired interaction, however, the $b c \bar{b} \bar{c}$ case might be favorable. In additional to studies about the $Q Q \bar{q} \bar{q}$ states, the authors also discussed the existence of $c c \bar{c} \bar{c}, b b \bar{b} \bar{b}$, and $b b \bar{c} \bar{c}$ tetraquarks in Ref. [287]. Because of lack of strongly separated mass scales, they concluded that no such bound states exist.

In a dynamical study with a nonrelativistic potential model in Ref. [180], the mass spectra of all types of full-heavy $Q_{1} Q_{2} \bar{Q}_{3} \bar{Q}_{4}(Q=c, b)$ tetraquark states were obtained. No bound states can be formed below the thresholds of any meson pairs $\left(Q_{1} \bar{Q}_{3}\right)-\left(Q_{2} \bar{Q}_{4}\right)$ or $\left(Q_{1} \bar{Q}_{4}\right)-\left(Q_{2} \bar{Q}_{3}\right)$, and thus such states with narrow widths are not expected in experiments. This conclusion about spectrum confirmed the arguments from the CMI estimations given in Ref. [110]. In a study with a nonrelativistic chiral quark model [295], the analysis of $b b \bar{b} \bar{b}$ states indicates that the $J^{P}=(0,1,2)^{+}$states are all higher than the corresponding thresholds, in both meson-meson and diquark-antidiquark configurations. The results also confirmed the conclusions from the CMI calculation with the method (18) [110].

In Ref. [148], Silvestre-Brac and Semay did not find any bound $Q Q \bar{Q} \bar{q}$ states with a nonrelativistic quark model, whose potential was proposed by Bhaduri et al [226]. This result is roughly consistent with the estimations with the CMI model (18) [77].

\section{$3.4 Q \bar{Q} q q q$ and $Q Q q q \bar{q}$}

Considering the quark delocalization color screening effects in a chiral quark model, Huang et al investigated the $P_{c}$-like hidden-charm pentaquarks in the molecule configuration in Ref. [296]. According to their results, the $P_{c}(4380)$ can be explained as the $\Sigma_{c}^{*} \bar{D}$ molecule with $J^{P}=3 / 2^{-}$. Other hiddencharm and hidden-bottom bound states were also investigated. But all the positive-parity states were found to be unbound. They studied the hidden-strangeness pentaquarks in Ref. [297]. In Ref. [298], the same group performed a dynamical calculation of the five-quark systems in the framework of a chiral quark model. The $P_{c}(4380)$ was again suggested to be a $\Sigma_{c}^{*} \bar{D}$ molecule. All the obtained positive-parity states are unbound in their calculation, unless the effective $\sigma$ meson exchange is employed. The authors did not adopt the assignment for the $P_{c}(4450)$ as the $\Sigma_{c} \bar{D}^{*}$ molecule because of the inconsistent parity, although their masses are close to each other. They presented a similar study of the hidden-bottom pentaquarks in Ref. [299].

In Ref. [300], the solution for the relativistic five-quark equations favors the assignment for the quantum numbers of the pentaquarks $P_{c}(4380)$ and $P_{c}(4450)$ to be $J^{P}=5 / 2^{+}$and $3 / 2^{-}$, respectively.

In the molecule picture, the authors of Ref. [301] studied the LHCb $P_{c}$ states with a chiral constituent quark model $[234,235]$ and confirmed the existence of several $\bar{D}^{(*)} \Sigma_{c}^{(*)}$ structures near the $P_{c}$ masses. In 
Ref. [302], the isospin-half $J^{P}=(1 / 2,3 / 2,5 / 2)^{-} u u d c \bar{c}$ pentaquarks with the color-octet $u d s$ component were investigated with the quark cluster model. Three structures were found to be around the $\Sigma_{c}^{(*)} \bar{D}^{(*)}$ thresholds: one bound state, two resonances, and one large cusp. The authors argued that the $P_{c}(4450)$ may arise from these structures.

In Ref. [303], the authors studied the possible pentaquark nature of the $P_{c}(4380)$ with $I\left(J^{P}\right)=$ $1 / 2\left(3 / 2^{-}\right)$using a hyperfine+confinement potential. With the variational method, they found that the ground state is the isolated $p$ and $J / \psi$ state, and the excited state lies far above the observed $P_{c}(4380)$. This observation led to the conclusion that the observed $P_{c}(4380)$ cannot be a compact $J^{P}=3 / 2^{-}$ pentaquark generated by the conventional two-body quark interactions. In Ref. [304], Stancu studied the effects of three-body chromoelectric interaction in pentaquark states and found that the three-body confining interaction can stabilize the pentaquark states with an appropriate sign. She suggested that perhaps the fall apart decay mode of the ground-state $n n n c \bar{c}$ pentaquarks in Ref. [303] may be avoided if such an interaction is included.

In Ref. [305], Richard, Valcarce, and Vijande studied the hidden-charm pentaquarks in a constituent model with the Grenoble AL1 potential by solving the five-body problem. They found that the lowest states with $(I, J)=(3 / 2,1 / 2)$ and $(3 / 2,3 / 2)$ are below their lowest $S$ - and $D$-wave thresholds, $\eta_{c} \Delta$ and $\Sigma_{c} \bar{D}$, and thus are expected to be stable. In a recent work [306], Hiyama, Hosaka, Oka, and Richard studied the five-body scattering problem with a nonrelativistic quark model by including explicitly open channels such as $J / \psi N, \eta_{c} N$, and $\Lambda_{c} \bar{D}$, etc. They also adopted the Grenoble potentials (AP1 and AL1). Their results are compatible with those obtained in Ref. [305]. According to their analysis, there does not exist any resonance at the energies of the LHCb $P_{c}$ states, while two narrow states are possible lying at $4690 \mathrm{MeV}$ and $4920 \mathrm{MeV}$ with $J^{P}=1 / 2^{-}$and $3 / 2^{-}$, respectively.

In Ref. [307], Stancu investigated the spectrum of the $u u d c \bar{c}$ hidden-charm pentaquark states with an $S U(4)$ Goldstone-boson-exchange model, in which the mass splittings are accounted for by the flavorspin hyperfine interaction. She found that the lowest positive-parity states with $J^{P}=1 / 2^{+}$and $3 / 2^{+}$ are below the lowest negative-parity state with $J^{P}=1 / 2^{-}$. The results accommodate a $3 / 2^{+} P_{c}(4380)$ and a $5 / 2^{+} P_{c}(4450)$.

With the Bethe-Salpeter equation, the spectrum of the heavy pentaquark states $Q \bar{Q} q q q(Q=c, b$, $q=u, d, s)$ were studied in the diquark-diquark-antiquark configuration in Ref. [308]. The masses of the $P_{c}$ like hidden-charm pentaquarks $J=3 / 2$ and $5 / 2$ states are $\sim 300 \mathrm{MeV}$ higher than the experimental values. The authors also calculated the masses of $Q Q q q \bar{q}$ states. The mass of the lowest $[c n][c n] \bar{n}$ state is around $4.54 \mathrm{GeV}$ which is larger than our result obtained in the CMI model [111].

In Ref. [274], the charmoniumlike pentaquark states were investigated in the dynamical diquark model by assuming that they are diquark-triquark states. Using the observed $P_{c}(4380)$ and $P_{c}(4450)$ as inputs, the authors predicted a number of other unobserved states. Especially the ground hidden-charm pentaquarks were below the $J / \psi N$ even $\eta_{c} N$ threshold.

The authors of Ref. [309] presented a study of the magnetic moments of the hidden-charm pentaquark states with the isospin $\left(I, I_{3}\right)=(1 / 2,1 / 2)$ and $J^{P}=(1 / 2 \sim 7 / 2)^{ \pm}$in the molecular, diquarktriquark, and diquark-diquark-antiquark models. The obtained magnetic moments are different, although the description for pentaquark masses and decay patterns can be achieved in all these models. Their results may be used to understand the inner structures and distinguish phenomenological models. In Ref. [310], the axial charges of the hidden-charm pentaquarks were studied in the chiral quark model. Their results can also be used to distinguish the underlying structures of the $P_{c}$ states.

\subsection{Color flux-tube model}

The flux-tube model [311, 312] was first extracted from the strong-coupling Hamiltonian lattice formulation [313] and the early descriptions of flux tubes as cylindrical bags of colored fields [314]. In the flux-tube model, the gluonic excitation was considered as the transverse vibration of the string- 
like flux tube between a quark-antiquark pair. The ordinary quark model was contained under the zero angular momentum approximation, while exotica can also be achieved for the non-zero angular momentum [312, 315].

The string potential in Hamiltonian was usually in a standard linear form to investigate the confinement phenomenon [315]. Such a flux-tube model has been extensively used to study the hadron masses, decays and production characteristics [315, 316, 317]. Recently, a naive color flux-tube model was developed by considering a harmonic-like confinement potential rather than a linear one to study the tetraquark states [318, 319].

In Ref. [320], Deng et. al. studied the lowest charged tetraquark states $Q q \bar{Q}^{\prime} \bar{q}^{\prime}(Q=c, b$ and $q=u, d, s)$ in the framework of the color flux-tube model with a four-body confinement potential. Within the color flux-tube model, the total hamiltonian of the diquark-antidiquark can be expressed as,

$$
\begin{aligned}
& H_{f}=\sum_{i=1}^{f}\left(m_{i}+\frac{p_{i}^{2}}{2 m_{i}}\right)-T_{C}+\sum_{i>j}^{f} V_{i j}+V_{\text {min }}^{C}(f), \\
& V_{i j}=V_{i j}^{B}+V_{i j}^{\sigma}+V_{i j}^{G},
\end{aligned}
$$

in which $f=2$ or $f=4, T_{C}$ is the center-of-mass kinetic energy, $p_{i}$ is the momentum of the $i$-th quark, $V_{\text {min }}^{C}(f)$ is the quadratic confinement potential. The interaction between two different quarks is described by $V_{i j}$, which contains the one-boson-exchange potential $V_{i j}^{B}$, the $\sigma$-meson exchange potential $V_{i j}^{\sigma}$, the one-gluon-exchange potential $V_{i j}^{G}$. In the diquark-antidiquark configuration, the energy of the tetraquark states $Q q \bar{Q}^{\prime} \bar{q}^{\prime}$ was then calculated by solving the four-body Schrödinger equation. The numerical results indicated that some compact resonance states were formed with the four-body quadratic potential. These resonances can not decay into $Q \bar{q}^{\prime}$ and $\bar{Q}^{\prime} q$ but into $Q \bar{Q}^{\prime}$ and $q \bar{q}^{\prime}$ through the breakdown and recombination of the flux tubes. The authors interpreted the charged states $Z_{c}(3900)$ and $Z_{c}(4025) / Z_{c}(4020)$ as the $S$-wave tetraquarks $[c u][\bar{c} \bar{d}]$ with quantum numbers $I=1$ and $J=1$ and 2 , respectively.

Later in Ref. [321], they systematically studied the charged $Z_{c}^{+}$family in the color flux-tube model and considered the spin-orbit interactions between quarks. Their investigation indicated that the $Z_{c}(3900) / Z_{c}(3885), Z_{c}(3930), Z_{c}(4025) / Z_{c}(4020), Z_{c}(4050), Z_{c}(4250)$, and $Z_{c}(4200)$ can be described as the $[c u][\bar{c} \bar{d}]$ tetraquark states with $n^{2 S+1} L_{J}$ and $J^{P}$ of $1^{3} S_{1}$ and $1^{+}, 2^{3} S_{1}$ and $1^{+}, 1^{5} S_{2}$ and $2^{+}, 1^{3} P_{1}$ and $1^{-}, 1^{5} D_{1}$ and $1^{+}, 1^{3} D_{1}$ and $1^{+}$, respectively. However, the two heavier charged states $Z_{c}^{+}(4430)$ and $Z_{c}^{+}(4475)$ can not be explained as tetraquark states in this model. In Ref. [322], Zhou, Deng and Ping also employed the flux-tube model to investigate the tetraquark states $c q \bar{c} \bar{q}$ with $I J^{P C}=01^{--}$. The vector states $Y(4008), Y(4140), Y(4260)$, and $Y(4360)$ were described as the $1^{1} P_{1}, 1^{5} P_{1}, 2^{5} P_{1}$ and $1^{5} F_{1}$ hidden-charm tetraquarks, respectively.

In Ref. [323], the hidden-charm pentaquark states were investigated within the framework of the color flux-tube model including a five-body confinement potential. The results showed that the main component of the $P_{c}^{+}(4380)$ can be described as a compact $u u d c \bar{c}$ pentaquark state with the pentagonal structure and $J^{P}=\frac{3}{2}^{-}$. However, the $P_{c}^{+}$(4450) state can not be interpreted as a compact pentaquark, since the masses of the positive parity $u$ udc $\bar{c}$ states were extracted much higher than those of the $P_{c}^{+}(4380)$ and $P_{c}^{+}(4450)$ in the color flux-tube model.

Recently, they also studied the stability of the doubly heavy tetraquark states $[Q Q][\bar{q} \bar{q}]$ in the color flux-tube model [324]. They solved the four-body Schrödinger equation to calculate the energies of all doubly heavy tetraquark states with both the color configurations $\left[[Q Q]_{\overline{\mathbf{3}}_{\mathbf{c}}}[\bar{q} \bar{q}]_{\mathbf{3}_{\mathbf{c}}}\right]_{\mathbf{1}}$ and $\left[[Q Q]_{\mathbf{6}_{\mathbf{c}}}[\bar{q} \bar{q}]_{\overline{\mathbf{6}}_{\mathbf{c}}}\right]_{\mathbf{1}}$. Their numerical results showed that the states $[b b][\bar{u} \bar{d}]$ with $I J^{P}=01^{+}$and $[b b]\left[\bar{q}^{\prime} \bar{s}\right]$ with $I J^{P}=\frac{1}{2} 1^{+}$ were the most promising stable doubly heavy tetraquark states against strong interaction. The doubly heavy tetraquark states $q q \bar{Q} \bar{Q}$ have also been addressed by a fully unitary and microscopic quark model with a triple string flip-flop potential [325]. They found several tetraquark bound states and resonances 
and calculated their masses and decay widths by computing the $T$ matrix and finding the pole positions in the complex energy plane.

\subsection{A short summary}

We summarize some interesting observations for the multiquark states from quark model investigations in recent years:

- The physical $X(3872)$ should be a mixed state due to the strong channel coupling between the bare quark model state $\chi_{c 1}(2 P)$ and the $D \bar{D}^{*}$ scattering state. Assigning the $X(3915)$ as the $\chi_{c 0}(2 P)$ charmonium is not supported by all quark model calculations. The $X(3940)$ can be assigned as the $\eta_{c}(3 S)$ or $h_{c}(2 P)$ (affected by $\left.D_{s} \bar{D}_{s}\right)$.

- In the charmonium or hybrid picture, the $X(4140)$ is a mixed $c \bar{c}$, a hybrid affected by $D_{s} \bar{D}_{s}^{*}$, or a cusp. The $X(4274)$ is the $\chi_{c 1}(3 P)$ affected by $D_{s}^{*} \bar{D}_{s}^{*}$. The $X(4350)$ is the $\chi_{c 0}(3 P)$ or a hybrid. The $X(4500)$ is the $\chi_{c 0}(4 P)$ or a hybrid. The $X(4700)$ is the $\chi_{c 0}(4 P)$ or $\chi_{c 0}(5 P)$.

- Perhaps both the mixed $4 S-3 D c \bar{c}$ states and the $D_{1} \bar{D}^{(*)}$ states contribute to the observed $Y(4260) / Y(4230)$ and $Y(4360)$, while the $Y(4630)$ and $Y(4660)$ are dominantly mixed $5 S-4 D$ even $3 D$ charmonia. Their masses can also be reproduced in tetraquark models.

- The masses of $Z_{c}(3900)$ and $Z_{c}(4020)$ may be obtained in both molecule picture and diquarkantidiquark picture. The $Z_{b}(10610)$ and $Z_{b}(10650)$ should be $B^{(*)} \bar{B}^{(*)}$ molecules, although the diquark-antidiquark interpretation for $Z_{b}(10650)$ is also possible. The nature of $Z_{c}(4430)$ is still unclear.

- Whether the lowest $c c \bar{u} \bar{d}$ tetraquark state is above or below the $D D^{*}$ threshold is still an open question, but the lowest $b b \bar{u} \bar{d}$ state should lie below the $B \bar{B}^{*}$ threshold.

- Few models favor the bound $c c \bar{c} \bar{c}$ and $b b \bar{b} \bar{b}$ states. A bound state in the $b c \bar{b} \bar{c}$ case might be favorable. Bound $Q Q \bar{Q} \bar{q}$ states should not exist.

- The quark model calculations favor the molecule interpretations for the $P_{c}(4380)$ and $P_{c}(4450)$.

\section{Meson exchange and scattering methods}

In the study of the hadronic molecules, almost all the meson-exchange models are constructed at the hadron level. One first derives the effective boson-exchange potentials in coordinate or momentum space, and then solves the bound state problem or scattering problem of two hadrons. From the obtained binding energy or scattering phase shifts, one extracts the resonance information. Such a formalism is a straightforward extension of the traditional meson exchange models in nuclear force.

There are meson-exchange models based on the quark-level interactions, e.g. based on the chiral quark model. After integrating out the inner degrees of freedom inside the hadrons, hadron-level effective meson-exchange potentials can be obtained. An example is illustrated in Ref. [326]. Of course, the molecule problems can also be dealt with at the quark level directly.

The color force of the van de Waals type is not described by the meson-exchange but by two-gluonexchange interactions. This type interaction was applied to understand the nature of the exotic states in the hadroquarkonium picture where a heavy quarkonium is embedded in a light hadronic matter. This hypothesis dated back to early nineties [327]. Although the interaction between ground-state hadrons may be weak and no bound state can be formed, probably such a force may result in bound states or resonances for radially and orbitally excited hadrons. 
In the chiral limit, the eight pseudoscalar meson masses vanish as the up, down and strangeness current quark masses go to zero. In contrast, the masses of the nucleon, rho meson or heavy mesons/baryons etc remain finite or even large in the chiral limit. They are sometimes denoted as the matter fields. If they contain the light quark degree of freedom, the matter fields will interact with the pseudoscalar mesons. The rigorous framework is the well-known chiral perturbation theory.

In order to ensure the chiral invariance, one should replace the simple derivative operator by the chirally covariant derivative operator in the construction of the kinetic energy of the matter fields at the leading order Lagrangian. The chirally covariant derivative operator contains the chiral connection. In other words, the kinetic term of the matter fields always induce the contact seagull term in the form of $M^{*} \frac{\pi \partial_{\mu} \pi}{F_{\pi}^{2}} M$, where the $M$ is the matter field, $\pi$ is the pseudoscalar meson, and $F_{\pi}$ is the pion decay constant. The model-independent seagull term is a direct manifestation of the chiral symmetry, which does not introduce new coupling constants.

Now we move on to the scattering process of the pseudoscalar meson and the matter fields. The physical $\pi M$ scattering amplitude is composed of the seagull amplitude, the corrections from higherorder tree-level diagrams and loop corrections. In other words, the seagull amplitude does not saturate the whole amplitude. Sometimes the seagull amplitude may be not dominant.

In the chiral unitary approach, only the seagull amplitude is iterated (unitarized) through the BetheSalpeter or Lippmann-Schwinger equation because of its easy-be-dealt-with and model-independent expression. From the pole positions of the unitarized amplitudes, one can extract information of bound states or resonances. Such states are called dynamically generated states in the literature. Since the seagull amplitude may miss some important physics as illustrated above, the chiral unitary approach sometimes generates spurious signals.

In the scattering of the light vector meson and the matter fields, one may treat the vector meson as the "gauge" field and introduce the hidden local symmetry into the Lagrangian. Then the chiral unitary formalism can be extended to the processes involving light vector mesons in a similar way.

If only the pions are exchanged between two heavy hadrons, the potentials can be organized order by order in the framework of the chiral perturbation theory $(\chi \mathrm{PT})$. One first constructs the tree-level Lagrangians order by order. Then one computes the higher order chiral corrections from the loop diagrams such as the triangle diagram, the box diagrams etc where only the two-particle irreducible contributions are kept. From the above scattering amplitudes, one derives the effective potentials at the given chiral order which enters the Schrödinger equation or Lippmann-Schwinger equation in the iteration. Again, one can extract the binding energy after solving the Schrödinger equation. One may also extract the resonance parameters either from the phase shifts or from the poles of the scattering amplitudes. This approach mimics the modern nuclear force in terms of $\chi \mathrm{PT}$ which is pioneered by Weinberg [328, 329].

\section{$4.1 Q \bar{Q} q \bar{q}$}

In Ref. [1], we performed a comprehensive review of the hidden-charm tetraquark states. In this section, we mainly introduce the theoretical progress on the systems with the $Q \bar{Q} q \bar{q}$ configuration after 2016. Different theoretical groups still focused on the interaction between a charmed meson and an anticharmed meson. The central issue remains to answer whether these observed $X Y Z$ charmoniumlike states can be understood in the hidden-charm molecular scenario.

In Ref. [330], the mass spectra for the heavy meson-antimeson bound states were discussed with the Hellmann plus one-pion-exchange potential. The results indicate that the $X(3872)$ is not a pure molecule. The authors of Ref. [331] discussed possible hadronic bound states formed by heavy-light mesons and heavy-light antimesons in the one-pion-exchange approach, where a heavy-light meson belongs to the $\left(0^{-}, 1^{-}\right)$multiplet or its chiral partner multiplet $\left(0^{+}, 1^{+}\right)$. The coupled channel results show that only the charm isosinglet molecules with $J^{P C}=1^{++}$are bound, which can be identified 
as the $X(3872)$. The analysis indicates that the reported $1^{+-}$isotriplet $Z_{c}(3900)^{ \pm}$is at best a near threshold resonance, and the $Z_{c}(4020)^{ \pm}$at best also a threshold effect. The study for the hidden-bottom meson-antimeson systems leads to the identification of the isotriplet exotic molecule with $J^{P C}=1^{+-}$ as a mixture of the $Z_{b}^{+}(10610)$ and $Z_{b}^{+}(10650)$. A bound isosinglet hidden-bottom molecule $X_{b}(10532)$ with $J^{P C}=1^{++}$analogous to the $X(3872)$ was proposed.

A recent systematic study of the possible hadronic molecules composed of the $S$-wave heavy-light mesons can be found in Ref. [332]. The assignment of the $X(3872)$ as a shallow $D \bar{D}^{*}$ molecule is supported, while assigning the $Z_{c}(3900), Z_{c}(4020), Z_{b}(10610)$, and $Z_{b}(10650)$ as molecules is not favorable. Moreover, the $Y(4140)$ cannot be assigned as a molecule composed of $D_{s}^{*+} D_{s}^{*-}$, neither.

With the Bethe-Salpeter equation, the authors of Ref. [333] calculated the mass of the $X(4140)$ in the molecule picture. They assigned this meson to be a mixed state of three pure molecule states $D^{* 0} \bar{D}^{* 0}, D^{*+} D^{*-}$, and $D_{s}^{*+} D_{s}^{*-}$. In Ref. [334], the $D \bar{D}^{*}$ molecule in the Bethe-Salpeter equation approach was discussed with the ladder and instantaneous approximations by considering $\sigma, \pi, \eta, \rho$, and $\omega$ exchanges. The $X(3872)$ can be a molecular bound state.

The possibility of the $X(4274)$ as a $P$-wave $D_{s} \bar{D}_{s 0}(2317)$ state in a quasipotential Bethe-Salpeter equation approach was discussed in Ref. [335]. A pole at $4275 \pm 11 i \mathrm{MeV}$ was produced through the $P$-wave interaction with the $J / \psi \phi$ channel. If this state can be interpreted as a hadronic molecule, an $S$-wave $D_{s} \bar{D}_{s 0}(2317)$ bound state below the $J / \psi \phi$ threshold should also exist.

In Ref. [336], He and Chen investigated the $D^{*} \bar{D}_{1}$ interaction in a quasipotential Bethe-Salpeter equation approach by considering one-pion-exchange interaction. They obtained a bound state at 4384 $\mathrm{MeV}$ with $I^{G}\left(J^{P C}\right)=0^{-}\left(1^{--}\right)$and a resonance at $4.461+i 39 \mathrm{MeV}$ with $I^{G}\left(J^{P}\right)=1^{+}\left(1^{+}\right)$. The former (latter) state was assigned to be the $Y(4390)$ (Z(4430)). Later in [337], Chen, Xiao, and He found that the molecule assignment for the $Y(4390)$ can naturally explain its observation in $e^{+} e^{-} \rightarrow \pi \pi h_{c}$ and its absence in $e^{+} e^{-} \rightarrow \pi \pi J / \psi$.

In a study with chiral unitary method in Ref. [338], Sakai, Roca, and Oset investigated the $(\bar{b} n)-(c \bar{n})$ and $(\bar{b} n)-(\bar{c} n)$ interactions. They found isoscalar bound states above $7 \mathrm{GeV}$ for the $0^{+} B D, 1^{+} B^{*} D$ and $B D^{*}$, and $(0,1,2)^{+} B^{*} D^{*}$ systems. In Ref. [339], the $D \bar{D}^{*}$ interaction was investigated with an extended hidden gauge symmetry in the chiral unitary model. A bound state slightly lower than the $D \bar{D}^{*}$ threshold was dynamically generated in the isoscalar channel, which was related to the $X(3872)$. An isoscalar $B \bar{B}^{*}$ bound state was also found.

These authors in Ref. [340] studied the $Z_{c}(3900)$ and $Z_{c}(4020)$ structures in a constituent quark model by considering the coupled-channels $D^{(*)} \bar{D}^{*}+h . c ., \pi J / \psi$, and $\rho \eta_{c}$. They found that these two structures are virtual states, whose effects as shown in the production line shapes can be seen as the $D^{(*)} \bar{D}^{(*)}$ threshold cusps.

In the hadrocharmonium picture, the authors of Ref. [341] studied possible $\psi(2 S) \phi$ bound states. They authors found that the obtained $S$-wave vector-vector bound state corresponds to a mass-degenerate multiples, and the $X(4274)$ can be such a state. The degeneracy indicates that two more structures around the $X(4274)$ should exist if this picture is correct. In Ref. [342], the bound states formed by $\eta_{c}$ or $J / \psi$ and isoscalar mesons in the hadrocharmonium picture were investigated. From their results, the $X(3915), X(3940), X(4160), Y(4260)$, and $Y(4360)$ are $\eta_{c} \eta^{\prime}, \eta_{c} f_{0}, \eta_{c} f_{1}, J / \psi f_{1}$, and $J / \psi f_{2}$ states, respectively. A different assignment was proposed in Ref. [343] to interpret the $X(3915), X(3940)$, and $X(4160)$ as dynamically generated resonances in the coupled channels of $D^{*} \bar{D}^{*}$ and $D_{s}^{*} \bar{D}_{s}^{*}$, with some relevant ones.

Voloshin interpreted the $Z_{c}(4100)$ and $Z_{c}(4200)$ as two states of hadrocharmonium related by the charm quark spin symmetry [344]. Later in Ref. [345], he predicted the existence of the strange hadrocharmonium resonances $Z_{c s}(4250)$ and $Z_{c s}(4350)$ which decay dominantly into $\eta_{c} K$ and $J / \psi K$, respectively.

In a study of the scattering problem related with near-threshold heavy-flavor resonances [346], the authors found that both the $D \bar{D}^{*}$ and other hadronic degrees of freedom are equally important inside 
the $Z_{c}(3900)$. The $D^{*} \bar{D}^{*}, D_{s} \bar{D}_{s}^{*}$, and $\Lambda_{c} \bar{\Lambda}_{c}$ components inside the $X(4020), X(4140)$, and $Y(4660)$, respectively, are not so important. For the $Y(4260)$, this study favors its interpretation as the $D_{1} \bar{D}$ molecule. A previous study with the same approach [347] indicates that both the $Z_{b}(10610)$ and $Z_{b}(10650)$ are dominated by the $B^{(*)} \bar{B}^{*}$ component, $76 \%$ and $68 \%$, respectively. See also discussions in Ref. [348].

\section{2 $Q Q \bar{q} \bar{q}$}

In Ref. [349], besides the $D_{s 2}^{*}(2573)$, the authors also studied the doubly charmed vector-vector meson states within the hidden gauge formalism in a coupled channel unitary approach. They obtained a pole around $3970 \mathrm{MeV}$ in the $I\left(J^{P}\right)=0\left(1^{+}\right) D^{*} D^{*}$ channel, which is $100 \mathrm{MeV}$ above the $D D^{*}$ threshold. Later in an extended work [338], the $(\bar{b} n)-(c \bar{n})$ and $(\bar{b} n)-(\bar{c} n)$ interactions were systematically investigated in order to see whether there are dynamically generated states. The authors noted that the $I\left(J^{P}\right)=0\left(1^{+}\right)$bound states in $B^{*} \bar{D}, B \bar{D}^{*}$, and $B^{*} \bar{D}^{*}$ systems are all possible. In Ref. [350], the doubly charmed exotic states as meson-meson molecules were investigated. The authors studied the scattering problem by solving the Lippmann-Schwinger equation, where the effective potentials were derived from the chiral constituent quark model [351]. Their results suggest the existence of a stable $Q Q \bar{q} \bar{q}$ state with $I\left(J^{P}\right)=0\left(1^{+}\right)$.

The authors of Ref. [352] presented a study of possible doubly charm and doubly bottom molecular states composed of a pair of heavy mesons, by solving the coupled Schrödinger equations through the one-boson-exchange (OBEP) method. The authors got bound and/or resonant states of various quantum numbers up to $J \leq 2$. In the $I\left(J^{P}\right)=0\left(1^{+}\right)$case, they obtained the $D D^{*}$ and $\bar{B} \bar{B}^{*}$ bound states with binding energies about tens of $\mathrm{MeV}$. In another coupled channel calculation for the molecule problem of $(Q \bar{q})-(Q \bar{q})(Q=c, b, q=u, d, s)$ systems in the OBEP framework [353], the $I\left(J^{P}\right)=0\left(1^{+}\right)$ $D D^{*}$ and $\bar{B} \bar{B}^{*}$ bound states were also obtained. In addition, the $I\left(J^{P}\right)=0\left(1^{+}\right) D \bar{B}^{*}$ molecule were found to be bound together with several other molecules of various configurations. Studies of their partner molecules can be found in Ref. [354].

Using the lattice potentials from Refs. [355, 356, 357] and the Born-Oppenheimer approximation, Bicudo et al studied the $u d \bar{b} \bar{b}$ tetraquark states and found an $I\left(J^{P}\right)=0\left(1^{+}\right)$state about $90 \mathrm{MeV}$ below the $B B^{*}$ threshold. Later in Ref. [358], an $I\left(J^{P}\right)=0\left(1^{-}\right)$resonance with $m=10576_{-4}^{+4} \mathrm{MeV}$ and $\Gamma=112_{-103}^{+90} \mathrm{MeV}$ was found. The authors of Ref. [359] discussed the possible states with the exotic doubly charmed configurations $D_{s 0}^{*}(2317) D$ and $D_{s 1}^{*}(2460) D^{*}$ based on the kaon-exchange interactions. The authors obtained bound states in the $J^{P}=0^{-}$and $2^{-}$channels.

In the framework of the chiral effective field theory, the authors of Ref. [360] derived the effective $D D^{*}$ potentials up to the order $\mathcal{O}\left(\epsilon^{2}\right)$, where $\epsilon$ can be the momentum of the pion, residual momentum of heavy mesons, or the $D-D^{*}$ mass splitting. As shown in Fig. 6, the two pion exchange contribution which is equivalent to phenomenological $\rho$ and $\sigma$ exchange contribution, was considered in their calculation. With the obtained potentials in the coordinate space, the authors found an $I\left(J^{P}\right)=0\left(1^{+}\right)$bound state by solving the Schrödinger equation. Further study in the same framework [361] showed that two $I\left(J^{P}\right)=0\left(1^{+}\right)$bound states, $\bar{B} \bar{B}^{*}$ and $\bar{B}^{*} \bar{B}^{*}$, are possible.

\section{$4.3 Q \bar{Q} q q q$}

In 2011, the authors of Ref. [195] predicted the existence of the hidden-charm molecular pentaquarks in the framework of one boson exchange model. Later, the hidden-charm $P_{c}(4380)$ and $P_{c}(4450)$ states were announced by LHCb in the $\Lambda_{b} \rightarrow J / \psi p K$ process [185], which inspired extensive discussions of the molecular pentaquarks with the $Q \bar{Q} q q q$ content. The research status of the hidden-charm molecular pentaquarks composed of an S-wave charmed baryon and an S-wave anti-charmed meson was covered in great details in Ref. [1]. Since the investigation along the same line continues, we discuss the progress in 


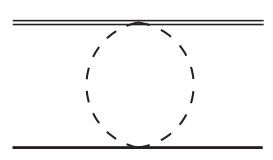

(c1)

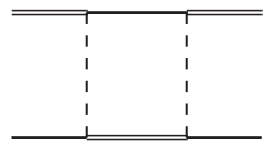

(c5)

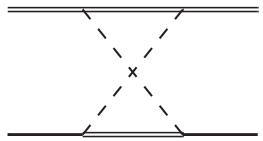

$(c 9)$

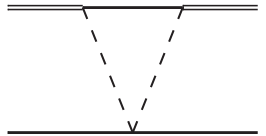

(c2)

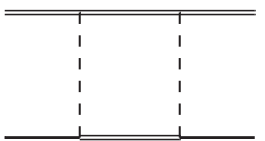

(c6)

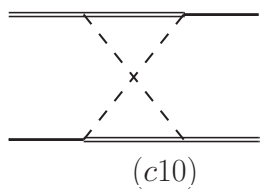

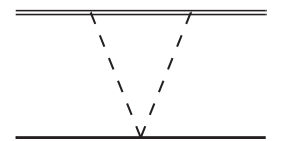

$(c 3)$

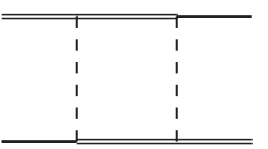

(c7)

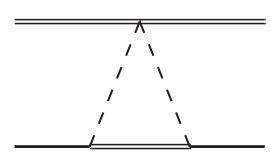

$(c 4)$

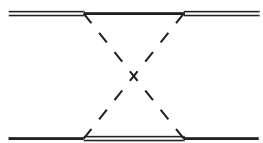

$(c 8)$

Figure 6: Two-pion-exchange diagrams at $O\left(\epsilon^{2}\right)$ for the $D D^{*}$ interaction. The solid, double-solid, and dashed lines stand for $D, D^{*}$, pion, respectively. Figure was taken from Ref. [360]

the past three years in this section. The coupled channel bound states problem of $\Sigma_{c}^{(*)} \bar{D}^{(*)}$ was studied with the one-pion-exchange model in Ref. [362]. The authors found the existence of one or two bound states with the binding energy of several $\mathrm{MeV}$ below the $\Sigma_{c}^{*} \bar{D}$ threshold. This result indicates that the $P_{c}(4380)$ can be understood as a loosely bound molecule. The extended analysis to the $b \bar{b}, b \bar{c}$, and $c \bar{b}$ cases also shows pentaquarks lying about $10 \mathrm{MeV}$ below the corresponding thresholds. For the $P_{c}(4450)$, it was proposed to be the Feshbach resonance. In Ref. [363], an extended study for the coupled system of $I\left(J^{P}\right)=\frac{1}{2}\left(\frac{3}{2}^{-}\right) J / \psi N-\Lambda_{c} \bar{D}^{(*)}-\Sigma_{c}^{(*)} \bar{D}^{(*)}$ with the complex scaling method was performed. The potential included both the pion exchange and $D^{(*)}$ meson exchange force. The contribution of the $J / \psi N$ channel was very small, and a pentaquark candidate with a value close to the $P_{c}(4380)$ was obtained.

In Ref. [364], the coupled ( $\bar{c} n)$-(cnn) meson-baryon systems were investigated in a one-mesonexchange potential model. From the obtained bound and resonant states with $I\left(J^{P}\right)=1 / 2(3 / 2,5 / 2)^{ \pm}$, the authors concluded that the $J^{P}$ assignments for the $P_{c}(4380)$ and $P_{c}(4450)$ are $3 / 2^{+}$and $5 / 2^{-}$, respectively, in agreement with the LHCb results. In Ref. [365], the study was extended to include coupling with compact five-quark channels and to the hidden-bottom case. The short-range effective potential from the compact five-quark channel was found to be attractive, which plays an important role in producing the $P_{c}$ states.

The author of Ref. [335] explored the $\bar{D}^{*} \Sigma_{c}$ interactions with a Bethe-Saltpeter equation method in the hadronic molecule picture by considering the channel coupling to $J / \psi p$. A pole near the $\bar{D}^{*} \Sigma_{c}$ threshold with $J^{P}=5 / 2^{+}$can be found. When this $P$-wave state is produced near threshold, the $S$-wave pole corresponding to $J^{P}=3 / 2^{-}$locates around $4390 \mathrm{MeV}$. These two poles are suggested to be related with the $P_{c}(4450)$ and $P_{c}(4380)$, respectively. Later in Ref. [366], by replacing the $\bar{c}$ quark with the $\bar{s}$ quark, the author interpreted the $N(1875)$ and $N(2100)$ as the strange molecular partners of the $P_{c}(4380)$ and $P_{c}(4450)$, respectively.

In Ref. [367], possible strange hidden-charm pentaquarks in the $\Sigma_{c}^{(*)} \bar{D}_{s}^{*}$ and $\Xi_{c}^{(\prime, *)} \bar{D}^{*}$ systems were investigated in a one-boson-exchange model. Promising candidates including $I\left(J^{P}\right)=0\left(1 / 2^{-}\right) \Xi_{c}^{\prime} \bar{D}^{*}$ and $0(1 / 2,3 / 2)^{-} \Xi_{c}^{*} \bar{D}^{*}$ states were predicted. This hidden-charm pentaquark with strangeness is accessible at $\mathrm{LHCb}$.

The authors of Ref. [368] discussed the scale invariance in hadron molecules like the $\Lambda_{c 1} \bar{D}-\Sigma_{c} \bar{D}^{*}\left(\Lambda_{c 1}\right.$ denotes $\left.\Lambda_{c}(2595)\right)$ coupled state, which is related to the $P_{c}(4450)$. The nearly on-shell pion exchange transition can generate a long-range $1 / r^{2}$ potential which can lead to approximate scale invariance for the equation describing the molecule if the attraction is strong enough. As a result, an Efimov-like geometrical spectrum in two-hadron systems is possible. The molecules, $\Lambda_{c 1} \bar{D}-\Sigma_{c} \bar{D}^{*}$ of $1 / 2^{+}, \Lambda_{c 1} D-\Sigma_{c} D^{*}$ 
of $1 / 2^{+}, \Lambda_{c 1} \bar{\Xi}_{b^{-}} \Sigma_{c} \bar{\Xi}_{b}^{\prime}$ of $0^{+} / 1^{-}$, and $\Lambda_{c 1} \Xi_{b^{-}} \Sigma_{c} \Xi_{b}^{\prime}$ of $1^{+}$, seem to satisfy the condition for the approximate scale invariance.

In Ref. [369], Chen, Hosaka, and Liu explored the intermediate- and short-range forces in the framework of one-boson-exchange model and studied various hadron-hadron bound state problems. They found $S$-wave $\Lambda_{c} \bar{D}, \Lambda_{c} B, \Lambda_{b} \bar{D}$, and $\Lambda_{b} B$ molecules. In the study of bound state problems in Ref. [370], the authors proposed a one-boson-exchange potential model by adding a screen Yukawa-like potential. From their results, the $P_{c}(4450)$ was interpreted as an $I\left(J^{P}\right)=1 / 2\left(3 / 2^{-}\right) \Sigma_{c} \bar{D}^{*}$ molecule.

The authors of Ref. [371] presented an exploratory study of possible $\bar{D} \Lambda_{c}-\bar{D} \Sigma_{c}$ and $B \Lambda_{c}-B \Sigma_{c}$ resonances by extending the Jülich-Bonn dynamical coupled-channel framework, which is a unitary meson-baryon exchange model. The authors found one pole in each partial wave up to $J^{P}=5 / 2^{ \pm}$in the hidden-charm case and several poles in partial waves up to $G_{17}$ in the hidden-bottom case. The very narrow pole with $J^{P}=1 / 2^{-}$in the hidden-charm case was interpreted as a $\bar{D} \Sigma_{c}$ bound state, which was predicted in Refs. [184, 193].

In Ref. [372], Huang and Ping investigated the hidden-charm and hidden-bottom pentaquark resonances in hadron-hadron scattering processes in the framework of the quark delocalization color screening model. They found a few narrow hidden-charm resonances above $4.2 \mathrm{GeV}$ and some narrow hiddenbottom states above $11 \mathrm{GeV}$. Besides, they also noticed bound $(Q \bar{Q})-N$ states from the behavior of the low-energy phase shifts obtained with coupled channel calculations.

In Ref. [373], the authors interpreted the $P_{c}(4450)$ as a $\psi(2 S) N$ bound state generated by the charmonium-nucleon interaction in terms of charmonium chromoelectric polarizabilities and the nucleon energy-momentum distribution. They obtained two almost degenerate narrow states at the position of the $P_{c}(4450)$ with $J^{P}=1 / 2^{-}$and $3 / 2^{-}$. The authors of Ref. [374] confirmed the results and predicted the isospin-3/2 $\psi(2 S) \Delta$ narrow bound states around $4.5 \mathrm{GeV}$ and broader resonances around $4.9 \mathrm{GeV}$ in the framework of the Skyrme model. Later in Ref. [375], with a QCD inspired approach, the authors interpreted the $P_{c}(4450)$ as a bound $\psi(2 S) N$ state with $J^{P}=3 / 2^{-}$, which is a member of one of two almost degenerate hidden-charm baryon octets. According to their study, one has to assign the $P_{c}(4380)$ as a $J^{P}=5 / 2^{+}$state but no natural interpretation was found in the hadroquarkonium picture. The authors also studied the other hadroquarkonia and compared their results with those in the one-pion exchange approach. They did not find any $\Upsilon(1 S) N$ bound state but found an inconclusive $\Upsilon(2 S) N$ state.

A study of the LHCb $P_{c}$ states in the hadroquarkonium picture was carried out in Ref. [327]. The authors solved the Schrödinger equation with the potential from QCD multiple expansion and got spectrum. The $P_{c}(4380)$ and $P_{c}(4450)$ can be interpreted as the $\psi(2 S) N$ and $\chi_{c 2}(1 P) N$ bound states, respectively. They also predicted the hadroquarkonium states in the hidden-bottom sector.

Unfortunately, no evidence of the exotic $\psi(2 S) p$ state was observed by LHCb in the $\Lambda_{b}^{0}$ decay into $\psi(2 S) p \pi^{-}[376]$. The hadrocharmonium picture is being challenged.

After the observation of $P_{c}(4380)$ and $P_{c}(4450)$, their possible inner structures were proposed either as tightly bound pentaquark states [210, 211, 377, 377, 378, 98, 100] or loosely bound molecular states [206, 207]. However, the experimental data in Ref. [185] was unable to distinguish these configurations. In 26 March 2019, at the Rencontres de Moriond QCD conference, the LHCb Collaboration reported the observation of three new pentaquarks [68]. The observed $P_{c}(4312)^{+}$may correspond to the $\Sigma_{c} \bar{D}$ molecule with $I\left(J^{P}\right)=1 / 2\left(1 / 2^{-}\right)$, while the $P_{c}(4440)^{+}$and $P_{c}(4457)^{+}$can be identified as the $\Sigma_{c} \bar{D}$ molecular states with $I\left(J^{P}\right)=1 / 2\left(1 / 2^{-}\right)$and $1 / 2\left(3 / 2^{-}\right)$. The current measurement strongly supports the molecular hidden-charm pentaquarks predicted in Refs. [195, 184, 193], which shall become a milestone in the exploration of the multiquark matter. After their announcement, these three pentaquark states were immediately studied in the QCD sum rules [379], the one-boson-exchange(OBE) model [380], a contact-range effective field theory and a contact-range effective field theory [381]. The isospin breaking decay pattern was studied in [382]. In Ref. [380], the authors studied these new pentaquarks in a direct calculation with the OBE model. Their result supported that the $P_{c}(4312)^{+}, P_{c}(4440)^{+}$and 
$P_{c}(4457)^{+}$may correspond to the loosely bound $\Sigma_{c} \bar{D}$ molecule with $\left(I=1 / 2, J^{P}=1 / 2^{-}\right), \Sigma_{c} \bar{D}^{*}$ with $\left(I=1 / 2, J^{P}=1 / 2^{-}\right)$and $\Sigma_{c} \bar{D}^{*}$ with $\left(I=1 / 2, J^{P}=3 / 2^{-}\right)$, respectively.

\section{$4.4 Q Q q q \bar{q}$}

In Refs. [205, 383], various baryon-meson molecular states were studied by considering vectormeson exchange forces as well as the coupled channel effects with the broken $S U(4)$ flavor symmetry. In the charm number $C=2$ sector, the authors predicted more than 10 states with various quantum numbers. The authors of Ref. [384] predicted a possible $\Xi_{c c} \bar{K}$ molecule, and the authors of Ref. [385] systematically studied dynamically generated double-charm baryons in a coupled channel unitary model. In a chiral unitary approach, the authors of Ref. [386] studied various dynamically generated doublecharm meson-baryon states through vector meson exchanges interactions. They found ten molecular pentaquark states.

In Ref. [387], the $\Lambda_{c} D$ and $\Lambda_{b} \bar{B}$ interactions and related bound state problems were studied. The authors derived two-pion-exchange potentials and regularized the divergence with a phenomenological cutoff. With solutions from the Schrödinger equation, they concluded that the $\Lambda_{b} \bar{B}$ bound state is possible, but the $\Lambda_{c} D$ bound state can only be prudently expected. In Ref. [369], Chen, Hosaka, and Liu explored the intermediate- and short-range forces in the framework of one-boson-exchange model and studied various hadron-hadron bound state problems. They found $S$-wave $\Lambda_{c} D, \Lambda_{c} \bar{B}, \Lambda_{b} D$, and $\Lambda_{b} \bar{B}$ molecules.

In addition to the study of the $I\left(J^{P}\right)=1 / 2\left(3 / 2^{-}\right)$coupled system $J / \psi N-\Lambda_{c} \bar{D}^{(*)}-\Sigma_{c}^{(*)} \bar{D}^{(*)}$ in Ref. [363], Shimizu and Harada also considered the coupled system $\Lambda_{c} D^{(*)}-\Sigma_{c}^{(*)} D^{(*)}$ in the $I\left(J^{P}\right)=1 / 2\left(3 / 2^{-}\right)$ channel. The authors used the same one-pion-exchange potential after the antimesons are replaced by mesons. They noted that there exists a doubly charmed baryon of the ccnn $\bar{n}$ type as a hadronic molecule, named $\Xi_{c c}^{*}(4380)$, whose mass and width are close to those of $P_{c}(4380)$.

The observation of the double-charm baryon $\Xi_{c c}(3621)$ by LHCb stimulated the investigations of the molecule-type $Q Q q q \bar{q}$ states in the literature. In Ref. [388], Guo considered the scattering of the $Q Q q$ baryons and Goldstone bosons in a chiral effective theory. According to their calculation, there are a pair of bound and virtual states near the $\Xi_{c c} K$ threshold, indicating that there exists an interesting exotic state with the quark component $c c u d \bar{s}$. A resonance pole around the $\Xi_{c c} \bar{K}$ threshold, a bound state below the $\Xi_{c c} \bar{K}$ threshold, and two resonances around the $\Xi_{c c} \pi$ and $\Omega_{c c} K$ thresholds were also found. The authors of Ref. [370] presented a study of various meson-baryon and baryon-baryon/antibaryon states in a one-boson-exchange potential model compensated by a screened Yukawa-like potential. A series of double-heavy molecules were obtained.

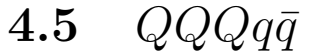

In addition to the molecular bound or resonant states with charm number $C=0, C=-1, C=1$, and $C=2$, the states with $C=3$ were also studied in Refs. [205, 383]. A flavor singlet bound state with $J^{P}=1 / 2^{-}\left(3 / 2^{-}\right)$with mass around $4.3(4.3) \mathrm{GeV}$ is possible, which results from the scattering of a $Q Q q$ baryon and a $Q \bar{q}$ meson as well as a $Q Q Q$ baryon and a $q \bar{q}$ meson. In the systematic study of triply charmed dynamically generated baryons in Ref. [385], the authors found one $J^{P}=1 / 2^{-}$bound state around $4.4 \mathrm{GeV}$ and one $J^{P}=3 / 2^{-}$bound state around $4.5 \mathrm{GeV}$ by coupling $(c c c)-(q \bar{q})$ and $(c c q)-(c \bar{q})$ channels.

The discovery of the charmonium-like $X Y Z$ states and $P_{c}(4380)$ and $P_{c}(4450)$ pushed the exploration of the hadronic molecular states in the past decade, which was based on the interaction between charmed hadron and anti-charmed hadron. The observation of the double-charm baryon $\Xi_{c c}^{++}(3621)$ drives us to further explore the interaction between the double-charm baryon and charmed meson, which is a natural extension of the one boson exchange model as shown in Fig. 7. The same idea was further 
extended to the investigation of the interaction between the double-charm baryon and charm baryon in Ref. [389].

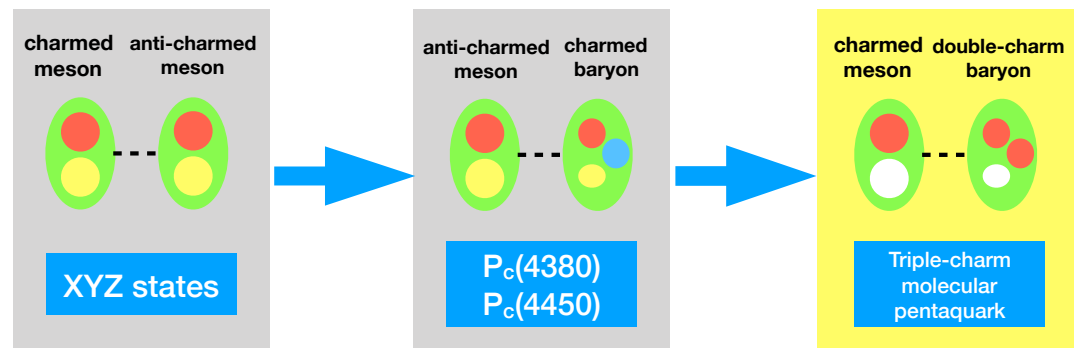

Figure 7: Evolution of interaction of hadrons and the corresponding connections with charmonium-like $X Y Z$ states, $P_{c}(4380) / P_{c}(4450)$ and triple-charm molecular pentaquark. Figure was taken from Ref. [390].

Due to the above motivation, in a one-boson-exchange potential model, possible molecular states formed by the $\Xi_{c c}(3621)$ and a charmed state $D$ and $D^{*}$ were discussed in Ref. [390]. Two isoscalar bound states, $\Xi_{c c} D$ of $1 / 2^{-}$and $\Xi_{c c} D^{*}$ of $3 / 2^{-}$, were found. Similar bound states with the same quantum numbers were found by replacing $D^{(*)}$ with $\bar{B}^{(*)}$. In Ref. [391], possible triple-charm molecular pentaquarks $\Xi_{c c} D_{1}$ and $\Xi_{c c} D_{2}^{*}$ were further discussed. The $I\left(J^{P}\right)=0\left(1 / 2^{+}, 3 / 2^{+}\right) \Xi_{c c} D_{1}$ and $I\left(J^{P}\right)=$ $0\left(3 / 2^{+}, 5 / 2^{+}\right) \Xi_{c c} D_{2}^{*}$ loosely bound molecules are possible.

\subsection{A short summary}

Since 2003, the observations of a series of charmonium-like near-threshold $X Y Z$ states have stimulated strong interest in the interactions between two heavy mesons. Later, the coupled-channel effect, various hyperfine interactions and recoil corrections were introduced into the one-boson-exchange model step by step. Within this simple framework, the hidden-charm molecular type pentaquarks were predicted. In 2015, LHCb collaboration did surprise us with two $P_{c}$ states. Very recently, LHCb collaboration updated their analysis with a ten times larger data sample, which strongly supports the molecular pentaquarks. At present, the one boson exchange model remains an effective and popular tool to deal with the hadronic interactions.

However, this framework sometimes lacks the definite predictive power because of too many unknown parameters such as the coupling constants and cutoff parameter. The original one-boson-exchange model was proposed for the nuclear force where there exists plenty of experimental data such as the deuteron binding energy and enough nucleon nucleon scattering data, which can be used to fix all the unknown model parameters. In contrast, except the pionic couplings, most of the light meson and heavy hadron interaction vertices remain unknown. Especially, the bound state or resonance solution is very sensitive to the cutoff parameter in the form factor which is introduced to suppress the ultraviolet contribution.

One may borrow the same formalism from the modern nuclear force and construct the interaction potential between two heavy hadrons in the framework of chiral perturbation theory. The pion exchange is still responsible for the long-range force. The two-pion exchange mimics the scalar meson and vector meson exchange to some extent. The contact heavy hadron interaction contributes to the short-range interaction in terms of the low energy constants which may be extracted through fitting to the lattice simulation data on the heavy hadron scattering if possible. One may expect significant progress along this direction in the future.

In short summary, many loosely bound molecular states or resonances such as the hidden-charm and open-strange pentaquark states and triple-charm pentaquark states have been proposed either within 
the one-boson-exchange model or with various unitary schemes, which may be searched for at $\mathrm{LHCb}$ and BelleII.

\section{$5 \quad$ Heavy quark symmetry and multiquark states}

QCD exhibits the chiral symmetry in the limit when quarks are massless, and the heavy quark symmetry [392] in the limit when quarks have infinitely large masses, both of which play important roles in understanding properties of hadrons and their interactions. The latter symmetry indicates both the heavy quark flavor symmetry (HQSS) which means that the dynamics is not affected by the exchange of heavy quark flavors, and the heavy quark spin symmetry (HQSS) which means that the dynamics is irrelevant of the heavy quark spin. For hadrons containing a single heavy quark, there exist degenerate multiplets which can be classified by the angular momentum of the light degree of freedom inside the hadrons. Recently, discussions about the application of the heavy quark symmetry to hadronic systems containing one heavy quark were performed in Ref. [393].

In the heavy quark limit, the heavy quark core of a hadron looks like a static color source, and the hadron resembles an atomic system. For conventional mesons with one heavy quark and conventional baryons with two heavy quarks, the heavy color source is of course in the representation $3_{c} / \overline{3}_{c}$. They can be related with the heavy antiquark-diquark symmetry (HADS) [394, 395, 396, 397] since the motion and spin interactions of the color source are negligible in this limit. An example of such relations is given in Ref. [397]

$$
M_{\Xi_{c c}^{*}}-M_{\Xi_{c c}}=\frac{3}{4}\left(M_{D^{*}}-M_{D}\right)
$$

For multiquark hadrons with two heavy quarks, the heavy quark core can also be in the representation $6_{c}$. One may naively expect that the lowest states contain the color- $\overline{3}_{c}$ heavy diquarks only, and then the doubly heavy tetraquarks can be related to the doubly heavy baryons. In fact, mass relations between $Q Q \bar{q} \bar{q}, Q Q q, Q q q$, and $Q \bar{q}$ states [164],

$$
M(Q Q \bar{q} \bar{q})-M(Q Q q)=M(Q q q)-M(Q \bar{q})
$$

can be found according to the heavy quark symmetry. In Ref. [397], other mass relations were given. Similarly, one may understand that relations exist between $Q Q q q \bar{q}$ pentaquarks and $Q q \bar{q} \bar{q}$ tetraquarks. With these heavy quark relations and heavy quark mass corrections, one may predict relevant multiquark masses.

The heavy quark symmetry may also be used to discuss hadronic molecule problem, where no additional light particles are created and the heavy hadron components remain distinct and move nonrelativistically [398]. From the existence of one molecule which consists of two or more heavy hadrons, one can expect possible existence of its partner molecules according to the heavy quark symmetry. The respective binding energies can be obtained by solving the Schrödinger equation.

\section{$5.1 Q \bar{Q} q \bar{q}$}

In Ref. [399], the exotic charmonium spectrum in hadron-charmonium, tetraquark, and hadronic molecule scenarios were investigated by employing heavy quark spin symmetry. The spectrum patterns from different models turn out to be quite distinct. For the $Y(4260)$, a lighter $0^{-+}$partner state around $4140 \mathrm{MeV}$ should exist only within the hadrocharmonium picture.

Three hadronic partner states of the $X(3872)$ with $J^{P C}=0^{++}, 1^{+-}$, and $2^{++}$should exist in the strict heavy-quark limit, if it is a $1^{++} D \bar{D}^{*}$ molecular state [400]. Once the one-pion-exchange interaction was included, this result was found to be robust only if all relevant channels and partial waves are 
considered. The heavy-quark spin symmetry implied spin partners of the $Z_{b}(10610)$ and $Z_{b}(10650)$, which were studied by assuming they are $B \bar{B}^{*}$ and $B^{*} \bar{B}^{*}$ molecules, respectively in Ref. [401]. With the force from short-range contact terms and the one-pion-exchange potential, the authors predicted the existence of an isovector $J^{P C}=2^{++}$tensor state lying a few $\mathrm{MeV}$ below the $B^{*} \bar{B}^{*}$ threshold.

In Ref. [402], the heavy quark symmetry partners of the $X(3872)$ were discussed by assuming that this exotic meson is a $D \bar{D}^{*}$ molecule. With the one-boson-exchange $(\pi, \sigma, \rho, \omega)$ potential model in which the cutoff parameter was extracted from $X(3872)$, the bound state problems of these meson-antimeson systems were discussed. The location and quantum numbers of the $Z_{b}(10610)$ and $Z_{b}(10650)$ were correctly reproduced. Other bound states such as $I\left(J^{P C}\right)=0^{+}\left(2^{++}\right) D^{*} \bar{D}^{*}, 0^{+}\left(1^{++}\right) B \bar{B}^{*}$, and $0^{+}\left(2^{++}\right)$ $B^{*} \bar{B}^{*}$ were predicted. We refer interested readers to Ref. [403], which introduced an effective Lagrangian implementing the heavy quark symmetry to describe those molecular states. See also Refs. [404, 405] where the non-relativistic effective theory was applied to describe heavy quarkonium hybrids .

In Ref. [406], Voloshin proposed the existence of light quark spin symmetry (LQSS) in molecular $Z_{b}$ resonances, $Z_{b}(10610) \sim\left|B^{*} \bar{B}, B \bar{B}^{*}\right\rangle$ and $Z_{b}(10650) \sim\left|B^{*} \bar{B}^{*}\right\rangle$, by noticing the weak coupling of the $Z_{b}(10650)$ to the $B^{*} \bar{B}+B \bar{B}^{*}$ channel. In the case of free heavy meson pairs, the spin structure of the two $Z_{b}$ states reads

$$
Z_{b}(10610) \sim \frac{1}{\sqrt{2}}\left(1_{b \bar{b}}^{-} \otimes 0_{q \bar{q}}^{-}+0_{b \bar{b}}^{-} \otimes 1_{q \bar{q}}^{-}\right), \quad Z_{b}(10650) \sim \frac{1}{\sqrt{2}}\left(1_{b \bar{b}}^{-} \otimes 0_{q \bar{q}}^{-}-0_{b \bar{b}}^{-} \otimes 1_{q \bar{q}}^{-}\right) .
$$

In the case of interacting heavy meson pairs, if the interaction in the state $1_{q \bar{q}}^{-}$differed from that in $0_{q \bar{q}}^{-}$, the two $Z_{b}$ states would not be the eigenstates for interaction mesons. Then, one has to assume that the interaction between the mesons does not depend on the total spin of the light quark-antiquark pair, which is the proposed LQSS. Unlike the HQSS, this approximate symmetry is not a symmetry from the underlying QCD, and is manifestly broken by the pion exchange force. Although the LQSS is unexpected, it was used to predict the existence of four new isovector negative $G$-parity resonances, $0^{+} B \bar{B}, 1^{+} B \bar{B}^{*}, 0^{+} B^{*} \bar{B}^{*}$, and $2^{+} B^{*} \bar{B}^{*}$, together with the HQSS. The application of this symmetry to the charm quark case would be complicated, because of the enhanced violation of HQSS and the breaking of isospin symmetry.

\section{$5.2 Q Q \bar{q} \bar{q}$}

In Ref. [164], Eichten and Quigg calculated the masses of the $Q Q \bar{q} \bar{q}(Q=c, b, q=u, d, s)$ tetraquark states based on mass relations obtained from the heavy quark symmetry and finite-mass corrections. According to their results, the $I\left(J^{P}\right)=0\left(1^{+}\right) c c \bar{u} \bar{d}$ state is $102 \mathrm{MeV}$ above the $D^{+} D^{* 0}$ threshold, while the $b b \bar{u} \bar{d}$ state is $121 \mathrm{MeV}$ below the $B^{-} \bar{B}^{* 0}$ threshold. The finding is consistent with that by Karliner and Rosner [166]. Besides, this calculation indicated a stable $b b \bar{n} \bar{s}$ bound state with $I\left(J^{P}\right)=0\left(0^{+}\right)$but no stable $b c \bar{u} \bar{d}$.

The chiral Lagrangian that uses HADS to relate doubly heavy tetraquarks to singly heavy baryons was given in Ref. [165], which is an extension of Ref. [407]. The author constructed the chiral Lagrangian for multiplets containing singly heavy mesons and doubly heavy baryons. With these Lagrangians and the measured mass of the $\Xi_{c c}$, Mehen studied properties of the five lowest-lying excited ccn states together with doubly heavy tetraquarks. He noted that the lowest $I\left(J^{P}\right)=0\left(1^{+}\right) b b \bar{u} \bar{d}$ state should be stable. If its mass is below $10405 \mathrm{MeV}$, he argued that the $I\left(J^{P}\right)=1\left(1^{+}\right) b b \bar{u} \bar{d}$ state should also be stable.

In Ref. [398], the authors presented a model-independent argument about the existence of near threshold exotic mesons containing two heavy quarks. Based on Born-Oppenheimer and semi-classical considerations, they found the existence of parametrically narrow tetraquarks, which are close to the threshold of two heavy mesons. 


\section{$5.3 \quad Q \bar{Q} q q q$ and $Q Q Q q \bar{q}$}

In Ref. [408], the structure of heavy quark spin multiplets for the $S$-wave $\bar{P}^{(*)} \Sigma_{Q}^{(*)}$ molecular states was studied. The authors introduced the light cloud spin (LCS, the allowed angular momentum of the light degree of freedom) basis, and constructed the unitary transformation matrices in relating the LCS basis and the molecule basis. They found four types of multiplets classified by the structure of heavy quark spin and LCS, and discussed their restrictions on decay widths of the molecules. In Ref. [409], they extended the formalism to study the heavy quark spin multiplet structures of the $P_{c^{-}}$like pentaquarks as $P$-wave hadronic molecules. Solving the coupled-channel Schrödinger equation in the OPEP model and comparing results with the previous study [408], they pointed out that it seems difficult to explain the masses and decay widths of the $P_{c}(4380)$ and $P_{c}(4450)$ simultaneously.

In Ref. [410], the authors discussed the possible partner state of the $P_{c}(4450)$ according to the heavy quark spin symmetry assuming it to be a $J^{P}=3 / 2^{-} \bar{D}^{*} \Sigma_{c}$ bound state. They predicted the existence of a $5 / 2^{-} \bar{D}^{*} \Sigma_{c}^{*}$ molecule with a binding energy similar to the $P_{c}(4450)$. In fact, before the observation of $P_{c}$ states by LHCb, the authors of Ref. [198] had discussed the heavy-quark spin multiplet structures in hidden-charm molecules.

Besides the hidden-charm partner state of the $P_{c}(4450)$, the authors of Ref. [410] also discussed the possible open-charm molecule partners of the $P_{c}(4450)$ using HADS. They predicted the $\Xi_{c c} \Sigma_{c}$ molecule with $J^{P}=0^{+}, \Xi_{c c} \Sigma_{c}^{*}$ molecule with $J^{P}=1^{+}, \Xi_{c c}^{*} \Sigma_{c}$ molecule with $J^{P}=2^{+}$, and $\Xi_{c c}^{*} \Sigma_{c}^{*}$ molecule with $J^{P}=3^{+}$. The binding energies of all these states are in the $20 \sim 30 \mathrm{MeV}$ range.

\section{QCD sum rules}

The formalism of QCD sum rules is a powerful and successful non-perturbative method [411, 412], which has been widely applied to study the mass spectra and decay properties of various exotic hadrons. Since we have thoroughly reviewed its applications to the hidden-charm pentaquark and tetraquark states in Ref. [1] and its applications to the open-charm tetraquark states in Ref. [413], we shall only briefly introduce the recent progress of this method in the present view, and we refer interested readers to Refs. [1, 413, 411, 412, 414, 415, 416, 417] for more discussions.

\subsection{A short induction to QCD sum rules}

A key idea of QCD sum rules is the quark-hadron duality, i.e., the equivalence of the (integrated) correlation functions at both the hadron level and the quark-gluon level.

When studying the mass spectrum of the hadron $H$, one calculates the two-point correlation function

$$
\Pi\left(q^{2}\right) \equiv i \int d^{4} x e^{i q x}\left\langle 0\left|\mathbb{T} \eta(x) \eta^{\dagger}(0)\right| 0\right\rangle,
$$

at both the hadron and quark-gluon levels. Here $\eta(x)$ is an interpolating current which has the same quantum numbers as the hadron $H$, and their coupling is defined to be

$$
\langle 0|\eta(0)| H\rangle \equiv f_{H}
$$

The interpolating current $\eta(x)$ can partly reflect the internal structure of the hadron $H$, but we still do not fully understand this relation $[418,419]$. We shall discuss this in details in the next subsection.

At the hadron level one expresses $\Pi\left(q^{2}\right)$ in the form of the dispersion relation:

$$
\begin{aligned}
\Pi\left(q^{2}\right) & =\frac{1}{\pi} \int_{s_{<}}^{\infty} \frac{\operatorname{Im} \Pi(s)}{s-q^{2}-i \varepsilon} d s \\
& \equiv \int_{s<}^{\infty} \frac{\rho_{\text {phen }}(s)}{s-q^{2}-i \varepsilon} d s
\end{aligned}
$$


where $\rho_{\text {phen }}(s) \equiv \operatorname{Im} \Pi(s) / \pi$ is the spectral density, for which one usually adopts a parametrization of one pole dominance for the ground state $H$ and a continuum contribution:

$$
\begin{aligned}
\rho_{\text {phen }}(s) & \equiv \sum_{n} \delta\left(s-M_{n}^{2}\right)\langle 0|\eta| n\rangle\left\langle n\left|\eta^{\dagger}\right| 0\right\rangle \\
& =f_{H}^{2} \delta\left(s-M_{H}^{2}\right)+\text { continuum } .
\end{aligned}
$$

At the quark-gluon level, one calculates $\Pi\left(q^{2}\right)$ using the method of operator product expansion $(\mathrm{OPE})$, and calculates the spectral density $\rho_{\mathrm{OPE}}(s)$ up to certain order in the expansion. After performing the Borel transformation at both the hadron and quark-gluon levels, and approximating the continuum using $\rho_{\mathrm{OPE}}(s)$ above a threshold value $s_{0}$, one obtains the sum rule equation

$$
\Pi\left(s_{0}, M_{B}^{2}\right) \equiv f_{H}^{2} e^{-M_{H}^{2} / M_{B}^{2}}=\int_{s_{<}}^{s_{0}} e^{-s / M_{B}^{2}} \rho_{\mathrm{OPE}}(s) d s,
$$

which can be used to calculate $M_{H}$ through

$$
M_{H}^{2}=\frac{1}{\Pi\left(s_{0}, M_{B}^{2}\right)} \frac{\partial \Pi\left(s_{0}, M_{B}^{2}\right)}{\partial\left(-1 / M_{B}^{2}\right)}=\frac{\int_{s_{<}}^{s_{0}} e^{-s / M_{B}^{2}} s \rho_{\mathrm{OPE}}(s) d s}{\int_{s_{<}}^{s_{0}} e^{-s / M_{B}^{2}} \rho_{\mathrm{OPE}}(s) d s} .
$$

The above two-point correlation function is investigated when extracting hadron masses, while one can also consider the three-point correlation function to study their decay properties:

$$
T_{A \rightarrow B C}\left(p, p^{\prime}, q\right)=\int d^{4} x d^{4} y e^{i p^{\prime} x} e^{i q y}\left\langle 0\left|\mathbb{T} \eta^{B}(x) \eta^{C}(y) \eta^{A \dagger}(0)\right| 0\right\rangle .
$$

Here $p, p^{\prime}$, and $q$ are the momenta of $A, B$, and $C$, respectively. The above correlation function can be used to study the $A \rightarrow B C$ decay. One still calculates it at both the hadron and quark-gluon levels.

At the hadron level one expresses $T_{A \rightarrow B C}\left(p, p^{\prime}, q\right)$ as:

$$
T_{A \rightarrow B C}\left(p, p^{\prime}, q\right)=g_{A \rightarrow B C} \times \frac{f_{A} f_{B} f_{C}}{\left(m_{A}^{2}-p^{2}\right)\left(m_{B}^{2}-p^{2}\right)\left(m_{C}^{2}-q^{2}\right)},
$$

where $g_{A \rightarrow B C}$ is the coupling constant, and $f_{A}, f_{B}$, and $f_{C}$ are the relevant decay constants.

At the quark-gluon level one calculates $T_{A \rightarrow B C}\left(p, p^{\prime}, q\right)$ using the method of operator product expansion (OPE). Again, by using the quark-hadron duality to relate the expressions of $T_{A \rightarrow B C}\left(p, p^{\prime}, q\right)$ at the hadron and quark-gluon levels, one can calculate the coupling constant $g_{A \rightarrow B C}$, and further evaluate the decay width of the $A \rightarrow B C$ process.

\subsection{Interpolating currents and their relations to physical states}

When applying the method of QCD sum rules to investigate a physical state, one always needs to construct the relevant interpolating current. However, we still do not fully understand their relations: the interpolating current sees only the quantum numbers of the physical state, so it can also couple to other physical states and thresholds having the same quantum numbers; while one can sometimes construct more than one interpolating currents, all of which couple to the same physical state.

In 2006 we first systematically constructed all the $u d \bar{s} \bar{s}$ interpolating currents of $J^{P C}=0^{++}$in a local product form, and found that there are five independent currents for this channel [420]. They can be either in the diquark-antidiquark form $([q q][\bar{q} \bar{q}])$

$$
\begin{aligned}
& S_{6}=\left(\bar{s}_{a} \gamma_{5} C \bar{s}_{b}^{T}\right)\left(u_{a}^{T} C \gamma_{5} d_{b}\right), \\
& V_{6}=\left(\bar{s}_{a} \gamma_{\mu} \gamma_{5} C \bar{s}_{b}^{T}\right)\left(u_{a}^{T} C \gamma^{\mu} \gamma_{5} d_{b}\right), \\
& T_{3}=\left(\bar{s}_{a} \sigma_{\mu \nu} C \bar{s}_{b}^{T}\right)\left(u_{a}^{T} C \sigma^{\mu \nu} d_{b}\right), \\
& A_{3}=\left(\bar{s}_{a} \gamma_{\mu} C \bar{s}_{b}^{T}\right)\left(u_{a}^{T} C \gamma^{\mu} d_{b}\right), \\
& P_{6}=\left(\bar{s}_{a} C \bar{s}_{b}^{T}\right)\left(u_{a}^{T} C d_{b}\right) .
\end{aligned}
$$


or in the meson-meson form $([\bar{q} q][\bar{q} q])$

$$
\begin{array}{ll}
S_{1}=\left(\bar{s}_{a} u_{a}\right)\left(\bar{s}_{b} d_{b}\right), & S_{8}=\left(\bar{s}_{a} \lambda_{a b}^{n} u_{b}\right)\left(\bar{s}_{c} \lambda_{c d}^{n} d_{d}\right), \\
V_{1}=\left(\bar{s}_{a} \gamma_{\mu} u_{a}\right)\left(\bar{s}_{b} \gamma^{\mu} d_{b}\right), & V_{8}=\left(\bar{s}_{a} \gamma_{\mu} \lambda_{a b}^{n} u_{b}\right)\left(\bar{s}_{c} \gamma^{\mu} \lambda_{c d}^{n} d_{d}\right), \\
T_{1}=\left(\bar{s}_{a} \sigma_{\mu \nu} u_{a}\right)\left(\bar{s}_{b} \sigma^{\mu \nu} d_{b}\right), & T_{8}=\left(\bar{s}_{a} \sigma_{\mu \nu} \lambda_{a b}^{n} u_{b}\right)\left(\bar{s}_{c} \sigma^{\mu \nu} \lambda_{c d}^{n} d_{d}\right), \\
A_{1}=\left(\bar{s}_{a} \gamma_{\mu} \gamma_{5} u_{a}\right)\left(\bar{s}_{b} \gamma^{\mu} \gamma_{5} d_{b}\right), & A_{8}=\left(\bar{s}_{a} \gamma_{\mu} \gamma_{5} \lambda_{a b}^{n} u_{b}\right)\left(\bar{s}_{c} \gamma^{\mu} \gamma_{5} \lambda_{c d}^{n} d_{d}\right), \\
P_{1}=\left(\bar{s}_{a} \gamma_{5} u_{a}\right)\left(\bar{s}_{b} \gamma_{5} d_{b}\right), & P_{8}=\left(\bar{s}_{a} \gamma_{5} \lambda_{a b}^{n} u_{b}\right)\left(\bar{s}_{c} \gamma_{5} \lambda_{c d}^{n} d_{d}\right),
\end{array}
$$

and can be related to each other through the Fierz transformation [421, 422, 423, 420]. In the above expressions, $a$ and $b$ are color indices, $C=i \gamma_{2} \gamma_{0}$ is the charge conjugation operator, and the superscript $T$ represents the transpose of Dirac indices. Note that only five of the ten currents in Eqs. (50) are independent, and all of them can be written as combinations of Eqs. (49). We refer to Ref. [420] for detailed discussion.

Later we applied the same method to systematically study various tetraquark, pentaquark, dibaryon and baryonium states [420, 424, 425, 426, 427, 428, 429, 430, 431, 432, 433, 434, 435, 436, 437, 438, $439,440,267]$. Since the internal structure of multiquark states is quite complicated, we also applied this method to systemically conventional mesons and baryons [441, 442, 443, 444, 445, 446, 447, 448, 449, 450, 451]. We clearly verified in many cases that the local diquark-antidiquark $([q q][\bar{q} \bar{q}])$ and meson-meson $([\bar{q} q][\bar{q} q])$ currents can be related to each other, so a straightforward conclusion is that the method of QCD sum rules can not actually differentiate the compact tetraquark and the hadronic molecule, when local four-quark currents are taken into account.

After so many QCD sum rule studies, we have obtained some experiences on hadronic interpolating currents and their relations to hadronic states, for examples,

1. The singly heavy baryons were systematically studied in Refs. [452, 453, 454, 455, 456, 457, 458, 459, 460], where we found that they have rich internal structures (see references of the review [413] for more relevant discussions). There can be as many as three $P$-wave excited $\Omega_{c}$ states of $J^{P}=1 / 2^{-}$, three of $J^{P}=3 / 2^{-}$, and one of $J^{P}=5 / 2^{-}$. For each state we constructed one relevant interpolating current. Maybe not all of them exist in nature, but the LHCb experiment [461] did observe as many as five excited $\Omega_{c}$ states at the same time, all of which are candidates of $P$-wave charmed baryons.

2. The mass spectra of vector and axial-vector hidden-charm tetraquark states were systematically investigated in Ref. [462], where we found as many as eight $q c \bar{q} \bar{c}(q=u / d)$ interpolating currents of $J^{P C}=1^{--}$. Comparably, there were also many vector charmonium-like states observed in particle experiments [35], including the $Y(4008)$ [463], $Y(4220)$ [464], $Y(4320)$ [63], $Y(4360)$ [465], $Y(4630)$ [466], and $Y(4660)$ [467], etc.

3. The hidden-charm pentaquark states having spin $J=\frac{1}{2} / \frac{3}{2} / \frac{5}{2}$ were systematically studied in Refs. [207, 468, 469], where we constructed hundreds of hidden-charm pentaquark interpolating currents. In 2015 the first two hidden-charm pentaquark states, $P_{c}(4380)$ and $P_{c}(4450)$, were discovered by $\mathrm{LHCb}[185]$. In this year $\mathrm{LHCb}$ discovered a new candidate $P_{c}(4312)$, and at the same time they separated the $P_{c}(4450)$ into two structures, $P_{c}(4440)$ and $P_{c}(4457)$ [68]. One may expect there would exist more hidden-charm pentaquark states to be discovered in future experiments.

The internal structures of (exotic) hadrons are not so simple. In QCD sum rule studies, we can construct relevant interpolating currents to partly reflect such internal structures, but their relations are even more complicated. 
To clarify this problem, we found a good subject in Ref. [418] that there are only two independent $s s \bar{s} \bar{s}$ tetraquark currents of $J^{P C}=1^{--}[470]$ :

$$
\begin{aligned}
& \eta_{1 \mu}=\left(s_{a}^{T} C \gamma_{5} s_{b}\right)\left(\bar{s}_{a} \gamma_{\mu} \gamma_{5} C \bar{s}_{b}^{T}\right)-\left(s_{a}^{T} C \gamma_{\mu} \gamma_{5} s_{b}\right)\left(\bar{s}_{a} \gamma_{5} C \bar{s}_{b}^{T}\right), \\
& \eta_{2 \mu}=\left(s_{a}^{T} C \gamma^{\nu} s_{b}\right)\left(\bar{s}_{a} \sigma_{\mu \nu} C \bar{s}_{b}^{T}\right)-\left(s_{a}^{T} C \sigma_{\mu \nu} s_{b}\right)\left(\bar{s}_{a} \gamma^{\nu} C \bar{s}_{b}^{T}\right),
\end{aligned}
$$

We can also construct four non-vanishing meson-meson $(\bar{s} s)(\bar{s} s)$ interpolating currents with $J^{P C}=1^{--}$, but they all depend on the above two diquark-antidiquark $(s s)(\bar{s} \bar{s})$ ones. Their relations can be derived by using the Fierz transformation [421, 422, 423, 420].

We have separately used $\eta_{1 \mu}$ and $\eta_{2 \mu}$ to perform QCD sum rule analyses in Ref. [470], by calculating the diagonal terms:

$$
\left\langle 0\left|T \eta_{1 \mu}(x) \eta_{1 \nu}^{\dagger}(0)\right| 0\right\rangle \quad \text { and } \quad\left\langle 0\left|T \eta_{2 \mu}(x) \eta_{2 \nu}^{\dagger}(0)\right| 0\right\rangle \text {. }
$$

The masses extracted are both around $2.3 \mathrm{GeV}$, which can be used to explain the $Y(2175)$ of $J^{P C}=1^{--}$.

To study the relations between interpolating currents and their relevant physical states, given that we do not know how to diagonalize physical states, in Ref. [418] we further calculated the following off-diagonal term

$$
\left\langle 0\left|T \eta_{1 \mu}(x) \eta_{2 \nu}^{\dagger}(0)\right| 0\right\rangle
$$

which were found to be non-zero, suggesting that $\eta_{1 \mu}$ and $\eta_{2 \mu}$ can couple to the same physical state. Based on Eqs. (53) and (54), two new currents were obtained in Ref. [418]

$$
\begin{aligned}
& J_{1 \mu}=\cos \theta \eta_{1 \mu}+\sin \theta i \eta_{2 \mu}, \\
& J_{2 \mu}=\sin \theta \eta_{1 \mu}+\cos \theta i \eta_{2 \mu},
\end{aligned}
$$

satisfying

$$
\left\langle 0\left|T J_{1 \mu}(x) J_{2 \nu}^{\dagger}(0)\right| 0\right\rangle \ll \sqrt{\left\langle 0\left|T J_{1 \mu}(x) J_{1 \nu}^{\dagger}(0)\right| 0\right\rangle\left\langle 0\left|T J_{2 \mu}(x) J_{2 \nu}^{\dagger}(0)\right| 0\right\rangle} .
$$

Hence, these two new currents are non-correlated, and should not strongly couple to the same physical state. In Ref. [418] we assumed they couple to two different states, whose masses were extracted to be

$$
\begin{aligned}
& M_{J_{1}}=2.41 \pm 0.25 \mathrm{GeV} \\
& M_{J_{2}}=2.34 \pm 0.17 \mathrm{GeV}
\end{aligned}
$$

with the mass splitting

$$
\Delta M=71_{-48}^{+172} \mathrm{MeV} .
$$

The mass extracted using $J_{2 \mu}$ is consistent with the experimental mass of the $Y(2175)$, suggesting that $J_{2 \mu}$ may couple to the $Y(2175)$; while the mass extracted using $J_{1 \mu}$ is a bit larger, suggesting that the $Y(2175)$ may have a partner state, whose mass is around $71_{-48}^{+172} \mathrm{MeV}$ larger. The latter may be used to explain the structure in the $\phi f_{0}(980)$ invariant mass spectrum at around $2.4 \mathrm{GeV}[471,43,472,44,473]$.

Later this method was applied in Ref. [419] to study the $s s \bar{s} \bar{s}$ tetraquark states with $J^{P C}=1^{+-}$. Again two independent interpolating currents were found, but any one of them was found to give reliable QCD sum rule results. The mass was extracted to be $2.00_{-0.09}^{+0.10} \mathrm{GeV}$, which can be used to explain the $X(2063)$ observed by BESIII recently [474].

In Ref. [475], the authors applied the same method to investigate four charged charmonium-like states at the same time, including the $Z_{c}(3900), Z_{c}(4020), Z_{c}(4430)$, and $Z_{c}(4600)$ [476]. Their results suggest that the $Z_{c}(3900)$ and $Z_{c}(4020)$ are two $S$-wave tetraquark states with $J^{P C}=1^{+-}$, and the other two higher states can be established as their first radial excitations; the $Z_{c}(3900)$ and $Z_{c}(4430)$ both contains one "good" diquark with $J^{P}=0^{+}$and one "bad" diquark with $J^{P}=1^{+}$, while the $Z_{c}(4020)$ and $Z_{c}(4600)$ both contains two "bad" diquarks with $J^{P}=1^{+}$; an illustration is given in Fig. 8 . 


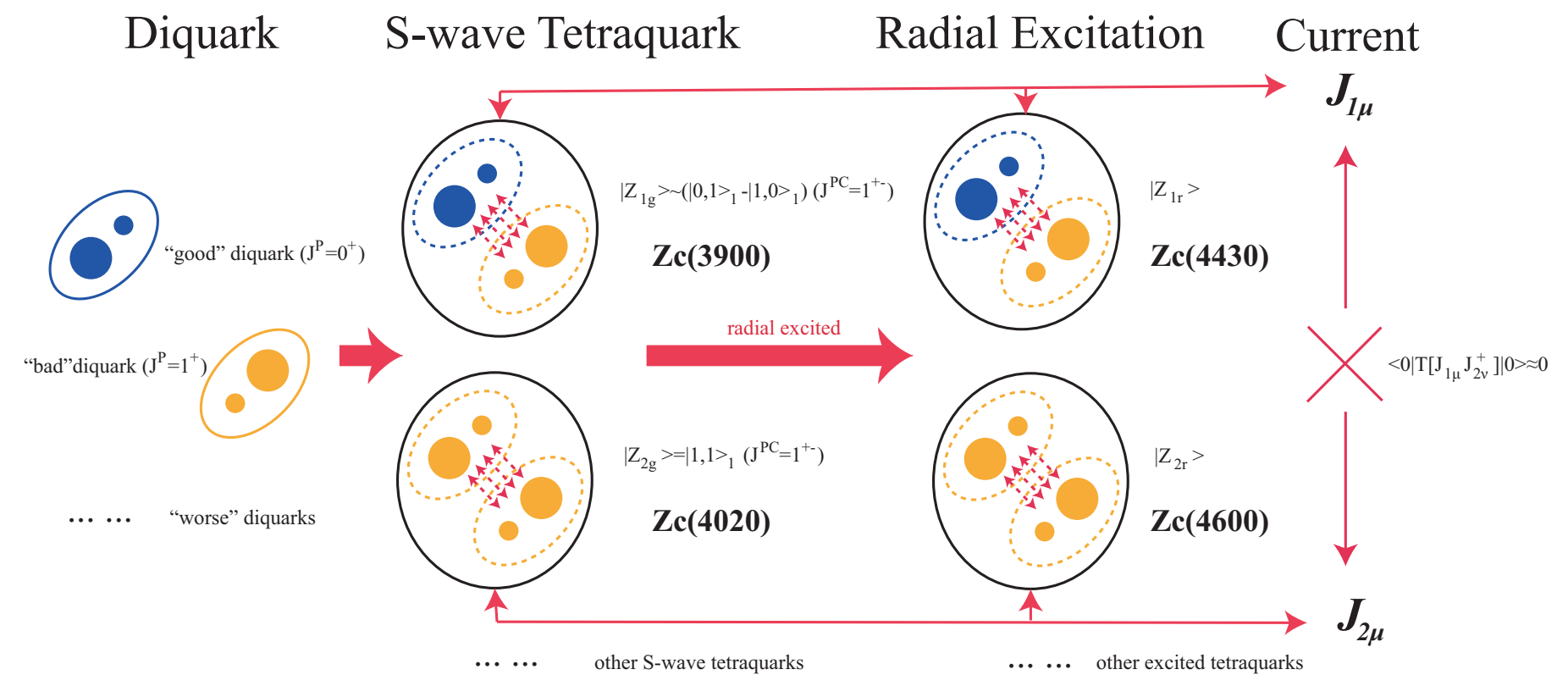

Figure 8: Possible interpretations of the $Z_{c}(3900), Z_{c}(4020), Z_{c}(4430)$, and $Z_{c}(4600)$, supported by a) the phenomenological analyses within the type II diquark-antidiquark model [97], and b) the QCD sum rule analyses performed in Ref. [475]. Taken from Ref. [475].

\section{3 $Q \bar{Q} q \bar{q}$ and $Q Q \bar{Q} \bar{Q}$}

Recently, the QCD sum rule method has been extensively used to study the tetraquark systems [477, 478, 479, 480, 481, 482, 483, 484, 485, 486, 487, 488, 489, 490, 491, 492, 493, 494, 495, 496, 497, 498, 499, $500,501,502,503]$. Most of these works have been already reviewed in Refs. [416, 1]. In this paper, we shall only introduce some very recent studies on the hidden-charm tetraquarks and pentaquarks. We will also introduce some investigations on the doubly, triply and fully charmed and bottom tetraquark states. These tetraquark systems are probably very stable and narrow against the strong decays, and thus motivated lots of theoretical interests. In this subsection we review the QCD sum rule studies on hidden-charm tetraquarks states in recent years.

To study the structures of the $X(3915), X(4350), X(4160)$ and $X^{*}(3860)$ in the diquark-antidiquark model, the authors of Ref. [504] investigated the hidden-charm and hidden-bottom $q c \bar{q} \bar{c}, s c \bar{s} \bar{c}, q b \bar{q} \bar{b}, s b \bar{s} \bar{b}$ tetraquark states with $J^{P C}=0^{++}$and $2^{++}$in the framework of QCD sum rules. They constructed ten scalar and four tensor interpolating currents to calculate the two-point correlation functions. After performing the numerical analyses, they obtained the mass spectra for the hidden-charm $q c \bar{q} \bar{c}$ and $s c \bar{s} \bar{c}$ tetraquark. As shown in Fig. 9, the mass of the $0^{++}$hidden-charm $q c \bar{q} \bar{c}$ tetraquark extracted from the currents $J_{4}(x), J_{9}(x)$ and $J_{10}(x)$ was about $3.8-3.9 \mathrm{GeV}$, which was consistent with the mass of the $X^{*}(3860)$ state. However, the tensor $q c \bar{q} \bar{c}$ tetraquark state was calculated to be around $4.06-4.16$, which was a bit higher than the mass of $X^{*}(3860)$. This implies that the assignment $J^{P C}=0^{++}$ is favored for $X^{*}(3860)$ than $2^{++}$, if it is a tetraquark. The same conclusion can be obtained for $X(3915)$. For $X(4160)$, their results cannot distinguish the scalar and tensor configurations, due to the nearly degenerated masses for them. It is also shown that the $X(4350)$ cannot be interpreted as a $s c \bar{s} \bar{c}$ tetraquark with either $J^{P C}=0^{++}$or $2^{++}$. The $X^{*}(3860)$ was also studied as a tetraquark state in Ref. [505].

In Ref. [267], the newly observed $X(4500)$ and $X(4700)$ were studied together with the $X(4140)$ and $X(4274)$ states, based on the diquark-antidiquark configuration in the framework of QCD sum rules. The authors found that the $X(4500)$ and $X(4700)$ can be both interpreted as $D$-wave tetraquark 


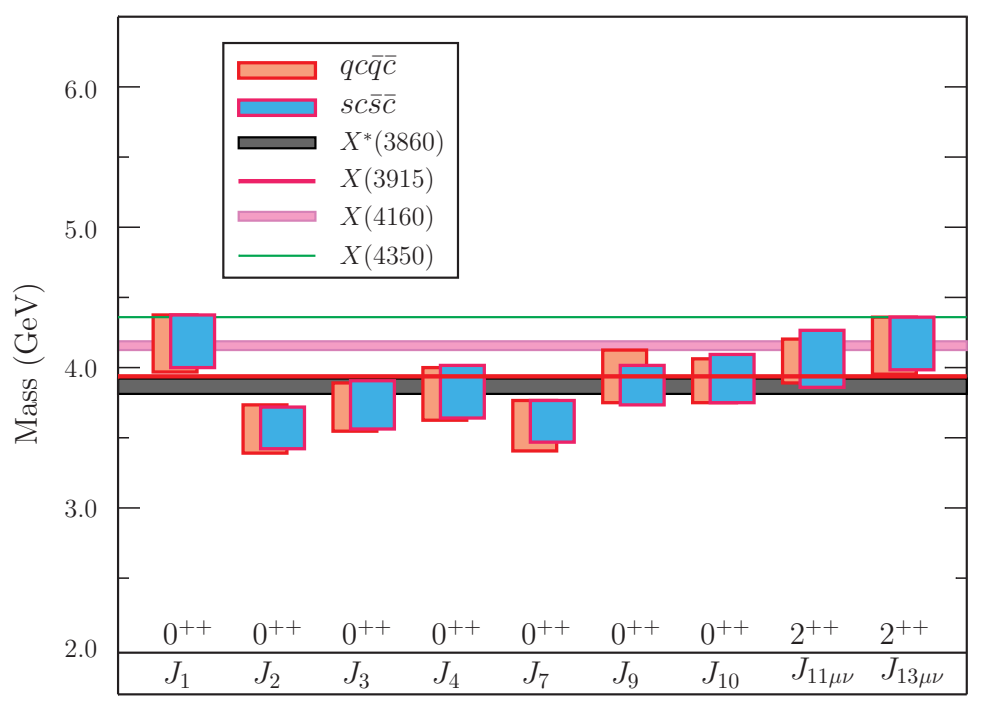

Figure 9: The mass spectra for the hidden-charm $q c \bar{q} \bar{c}$ and $s c \bar{s} \bar{c}$ tetraquark states with $J^{P C}=0^{++}$and $2^{++}$, taken from Ref. [504]

states with the quark content $\operatorname{cs} \bar{c} \bar{s}$ and quantum numbers $J^{P}=0^{+}$: the $X(4500)$ consists of one $D$-wave "bad" diquark and one $S$-wave "bad" antidiquark, with the antisymmetric color structure $\left[\overline{\mathbf{3}}_{\mathbf{c}}\right]_{c s} \otimes\left[\mathbf{3}_{\mathbf{c}}\right]_{\bar{c} \bar{s}}$; the $X(4700)$ consists of similar diquarks, but with the symmetric color structure $\left[\mathbf{6}_{\mathbf{c}}\right]_{c s} \otimes\left[\overline{\mathbf{6}}_{\mathbf{c}}\right]_{\bar{c} \bar{s}}$. These two interpretations were remarkably similar to those obtained in Ref. [462], in which the $X(4140)$ and $X(4274)$ can be both interpreted as $S$-wave $c s \bar{c} \bar{s}$ tetraquark states of $J^{P}=1^{+}$, but with distinct color structures. They also calculated the masses of the hidden-bottom partner states of the $X(4500)$ and $X(4700)$ and suggested to search for them in the $\Upsilon \phi$ invariant mass distribution. The masses and decay widths for the $X(4500), X(4700), X(4140)$ and $X(4274)$ mesons were also studied as the $c s \bar{c} \bar{s}$ tetraquark states in Refs. [506, 507, 508] and molecule state in Ref. [509] by using QCD sum rules.

The QCD sum rule has also been performed to study the doubly hidden-charm/bottom tetraquark states in Ref. [510], in which the moment method was adopted instead of the Borel transformation. The moment was defined as the $n$-th derivative of the correlation function $\Pi\left(Q^{2}\right)$ in the Euclidean region

$$
\begin{aligned}
M_{n}\left(Q_{0}^{2}\right) & =\left.\frac{1}{n !}\left(-\frac{d}{d Q^{2}}\right)^{n} \Pi\left(Q^{2}\right)\right|_{Q^{2}=Q_{0}^{2}} \\
& =\int_{16 m_{Q}^{2}}^{\infty} \frac{\rho(s)}{\left(s+Q_{0}^{2}\right)^{n+1}} d s=\frac{f_{X}^{2}}{\left(m_{X}^{2}+Q_{0}^{2}\right)^{n+1}}\left[1+\delta_{n}\left(Q_{0}^{2}\right)\right],
\end{aligned}
$$

where $\rho(s)$ is the spectral function and the narrow resonance approximation was adopted in the last step. The $\delta_{n}\left(Q_{0}^{2}\right)$ in Eq. (60) contains the contributions of higher states and the continuum. It tends to zero as $n$ goes to infinity for a certain value of $Q_{0}^{2}$. Then the hadron mass of the lowest lying resonance can be obtained as

$$
m_{X}=\sqrt{\frac{M_{n}\left(Q_{0}^{2}\right)}{M_{n+1}\left(Q_{0}^{2}\right)}-Q_{0}^{2}} .
$$

In Refs. [510, 511], the authors studied both the $c c \bar{c} \bar{c}$ and $b b \bar{b} \bar{b}$ tetraquark systems with various quantum numbers. As shown in Fig. 10, all the $b b \bar{b} \bar{b}$ tetraquark states were predicted to be slightly below than the mass thresholds of $\eta_{b}(1 S) \eta_{b}(1 S)$ and $\Upsilon(1 S) \Upsilon(1 S)$, while the $c c \bar{c} \bar{c}$ tetraquarks lied above the two-charmonium thresholds $\eta_{c}(1 S) \eta_{c}(1 S)$ and $J / \psi J / \psi$. Moreover, the positive parity states with $J^{P C}=0^{++}, 1^{++}, 1^{+-}, 2^{++}$were lighter than the negative parity states. 
In Refs. [512, 513], the $c c \bar{c} \bar{c}$ and $b b \bar{b} \bar{b}$ tetraquark states with $J^{P C}=0^{++}, 1^{+-}, 1^{--}, 2^{++}$were also studied in the Borel sum rule method. The extracted masses for these tetraquarks were slightly different with those obtained in Ref. [510].

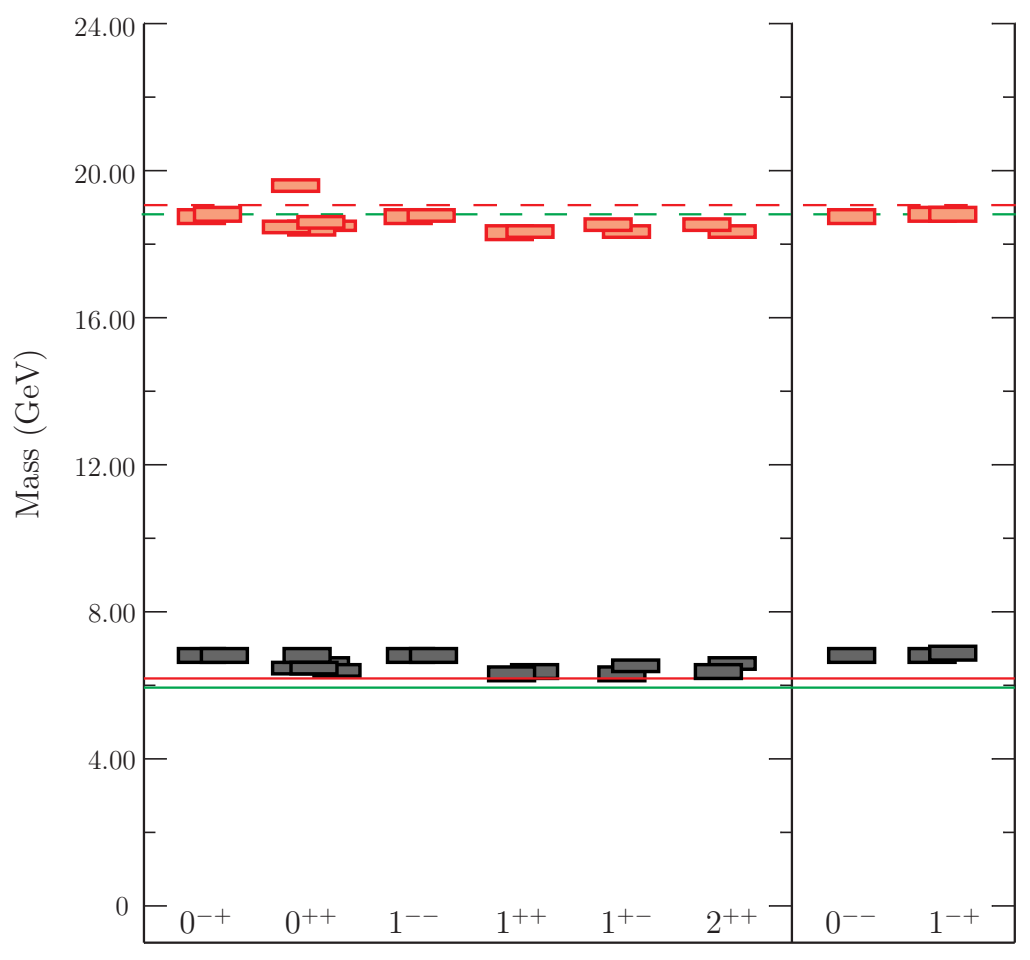

Figure 10: The tetraquark mass spectra for the $c c \bar{c} \bar{c}$ and $b b \bar{b} \bar{b}$ systems, taken from Ref. [510]. The green and red solid (dashed) lines indicate the two-charmonium (bottomonium) thresholds $\eta_{c}(1 S) \eta_{c}(1 S)$ $\left(\eta_{b}(1 S) \eta_{b}(1 S)\right)$ and $J / \psi J / \psi(\Upsilon(1 S) \Upsilon(1 S))$, respectively.

\section{4 $Q Q \bar{q} \bar{q}$ and $Q Q \bar{Q} \bar{q}$}

The triply heavy tetraquark states $Q Q \bar{Q} \bar{q}$ with $J^{P}=0^{+}$and $1^{+}$were also studied in Ref. [514], including the explicitly exotic states $c c \bar{b} \bar{q}$ and the hidden exotic states $c c \bar{c} \bar{q}, b b \bar{b} \bar{q}, c b \bar{b} \bar{q}$. The masses are lower than those estimated with the CMI model [107]. Especially, the $b b \bar{b} \bar{q}$ states were predicted to lie below the bottomonium plus $B^{(*)}$ thresholds and their OZI(Okubo-Zweig-Iizuka)-allowed strong decays are kinematically forbidden. These tetraquarks were expected to be very narrow and the authors suggested to search for them in the final states with a $B$ meson plus a light meson or photon processes. More studies are needed to understand such triple-heavy tetraquark states.

In Ref. [515], the mass spectra for the doubly charmed/bottom $Q Q \bar{q} \bar{q}, Q Q \bar{q} \bar{s}, Q Q \bar{s} \bar{s}$ tetraquark states with quantum numbers $J^{P}=0^{-}, 0^{+}, 1^{-}$and $1^{+}$have been calculated. Their numerical results indicated that the masses of the doubly bottom tetraquarks $b b \bar{q} \bar{q}, b b \bar{q} \bar{s}, b b \bar{s} \bar{s}$ were below the corresponding thresholds of the two-bottom mesons, two-bottom baryons, and one doubly bottom baryon plus one anti-nucleon. These doubly bottom tetraquarks are thus predicted to be stable against the strong decays. These results were consistent with the earlier study in Ref. [516]. Further in Ref. [517], the open-flavor scalar and axial-vector $b c \bar{q} \bar{q}$ and $b c \bar{s} \bar{s}$ tetraquark states were also studied and their masses were below the $D^{(*)} \bar{B}^{(*)}$ and $D_{s}^{(*)} \bar{B}_{s}^{(*)}$ thresholds respectively, suggesting dominantly weak decay mechanisms.

Later, the doubly heavy tetraquark states have attracted lots of attention [518, 519, 520, 521], motivated by the discovery of doubly charmed baryon $\Xi_{c c}^{++}$. In Ref. [519], the authors calculated 
both the masses and two-body strong decay width for the $c c \bar{s} \bar{s}$ and $c c \bar{d} \bar{s}$ tetraquarks with $J^{P}=0^{-}$. They obtained $m_{T}=(4390 \pm 150) \mathrm{MeV}$ and $\Gamma=(302 \pm 113) \mathrm{MeV}$ for the $\operatorname{cc} \bar{s} \bar{s}$ tetraquark, while $\tilde{m}_{T}=(4265 \pm 140) \mathrm{MeV}$ and $\tilde{\Gamma}=(171 \pm 52) \mathrm{MeV}$ for the $c c \bar{d} \bar{s}$ tetraquark. Since the masses of the doubly bottomed tetraquark were predicted below the two-meson thresholds, the weak decays of the axial-vector $T_{b b \bar{u} \bar{d}}^{-}$state to the scalar state $Z_{b c \bar{u} \bar{d}}^{0}$ were investigated using the three-point sum rule approach [520]. They recalculated the spectroscopic parameters of the $T_{b b \bar{u} \bar{d}}^{-}$and $Z_{b c \bar{u} \bar{d}}^{0}$ states in the framework of two-point QCD sum rules. The semileptonic decay channels $T_{b b \bar{u} \bar{d}}^{-} \rightarrow Z_{b c \bar{u} \bar{d}}^{0} l \bar{\nu}_{l}(l=e, \nu, \tau)$ were then investigated with the width and lifetime $\Gamma=(7.17 \pm 1.23) \times 10^{-8} \mathrm{MeV}$ and $\tau=9.18_{-1.34}^{+1.90}$ fs. Later in Ref. [521], they also studied the semileptonic decays of the scalar tetraquark $Z_{b c \bar{u} \bar{d}}^{0}$ to final states $T_{b s \bar{u} \bar{d}}^{-} e^{+} \nu_{e}$ and $T_{b s \bar{u} \bar{d}}^{-} \mu^{+} \nu_{\mu}$. After reanalysing the spectroscopic parameters of $T_{b s \bar{u} \bar{d}}^{-}$, they used the three-point sum rules to evaluate the weak form factors and extrapolated them to the whole momentum region. The total semileptonic decay width and lifetime of the scalar tetraquark $Z_{b c \bar{u} \bar{d}}^{0}$ were finally obtained as $\Gamma=(2.37 \pm 0.36) \times 10^{-11} \mathrm{MeV}$ and $\tau=27.8_{-3.7}^{+4.9} \mathrm{ps}$.

\section{5 $Q \bar{Q} q q q$}

Since the discovery of the $P_{c}(4380)$ and $P_{c}(4450)$ by LHCb in 2015 [185], there have been lots of theoretical studies using various methods, and the QCD sum rule approach is one of the most popular one.

In Ref. [207], we applied the method of QCD sum rules and studied the $P_{c}(4380)$ and $P_{c}(4450)$ as exotic hidden-charm pentaquarks composed of an anti-charmed meson and a charmed baryon. Our results suggest that the $P_{c}(4380)$ and $P_{c}(4450)$ have quantum numbers $J^{P}=3 / 2^{-}$and $5 / 2^{+}$, respectively. We also predicted the masses of their hidden-bottom partners to be $11.55_{-0.14}^{+0.23} \mathrm{GeV}$ and $11.66_{-0.27}^{+0.28} \mathrm{GeV}$. This study was later expanded in Refs. [468, 469], where we found two mixing currents, and again our results suggest that they can be identified as hidden-charm pentaquark states having $J^{P}=3 / 2^{-}$and $5 / 2^{+}$, while there still exist other possible spin-parity assignments, such as $J^{P}=3 / 2^{+}$and $5 / 2^{-}$, which still needs to be clarified in further theoretical and experimental studies.

Especially, in Ref. [468] we systematically constructed all the possible local hidden-charm pentaquark currents with spin $J=\frac{1}{2} / \frac{3}{2} / \frac{5}{2}$ and quark contents $u u d c \bar{c}$, through which we found that the internal structure of hidden-charm pentaquark states is quite complicated. We derived some mass predictions, and we summarized in Table 5 all the mass predictions that are extracted from single currents and less than $4.5 \mathrm{GeV}$. The coincidence of these mass predictions with the masses of $P_{c}(4312), P_{c}(4440)$, and $P_{c}(4457)$ measured in the recent LHCb experiment [68] drives us to the "molecular" picture [379] that the $P_{c}(4312)$ can be well explained as the $\left[\Sigma_{c}^{++} \bar{D}^{-}\right]$bound state with $J^{P}=1 / 2^{-}$; the $P_{c}(4440)$ and $P_{c}(4457)$ can be explained as the $\left[\Sigma_{c}^{*++} \bar{D}^{-}\right]$and $\left[\Sigma_{c}^{+} \bar{D}^{* 0}\right]$ bound states with $J^{P}=3 / 2^{-}$, respectively, while one of them may also be explained as the $\left[\Sigma_{c}^{+} \bar{D}^{0}\right]$ bound state with $J^{P}=1 / 2^{-}$or the $\left[\Sigma_{c}^{*+} \bar{D}^{* 0}\right]$ bound state with $J^{P}=5 / 2^{-}$; there is still a place for the $P_{c}(4380)$, i.e., to be explained as the $\left[\Sigma_{c}^{++} \bar{D}^{*-}\right]$ bound state with $J^{P}=3 / 2^{-}$. The $\left[\Sigma_{c} \bar{D}^{*}\right]$ bound state with $J^{P}=1 / 2^{-}$was not investigated in Ref. [468]. See Ref. [379] for detailed discussions of these possible interpretations.

In Ref. [522], Azizi et al performed QCD sum rules analyses on the hidden-charm pentaquark states with $J^{P}=3 / 2^{ \pm}$and $5 / 2^{ \pm}$. They adopted a molecular picture for $J^{P}=3 / 2^{ \pm}$states and a mixed current in a molecular form for $5 / 2^{ \pm}$, and their analyses suggested that the $P_{c}(4380)$ and $P_{c}(4450)$ can be considered as hidden-charm pentaquark states with $J^{P}=3 / 2^{-}$and $5 / 2^{+}$, respectively. Later in Ref. [523], they applied the same method to study the hidden-bottom pentaquark states with spin $J=3 / 2$ and $5 / 2$, and predicted their masses. The strong decays and electromagnetic multipole moments of the $P_{c}^{+}(4380)$ were later studied in Refs. [524, 525, 526].

In Ref. [527], Wang constructed the diquark-diquark-antiquark type interpolating currents, and his QCD sum rule studies also supported to assign the $P_{c}(4380)$ and $P_{c}(4450)$ as $J^{P}=3 / 2^{-}$and $5 / 2^{+}$ pentaquark states, respectively. This study was later expanded in Refs. [528, 529, 530]. 
Table 5: Mass predictions for the hidden-charm pentaquark states with spin $J=\frac{1}{2} / \frac{3}{2} / \frac{5}{2}$ and quark contents $u u d c \bar{c}$ [468]. They are extracted using hidden-charm pentaquark currents composed of colorsinglet charmed baryon fields and anti-charmed meson fields, i.e., $\left[\epsilon^{a b c} u_{a} d_{b} c_{c}\right]\left[\bar{c}_{d} u_{d}\right]$ or $\left[\epsilon^{a b c} u_{a} u_{b} c_{c}\right]\left[\bar{c}_{d} d_{d}\right]$, where the subscripts $a, b, c, d$ are color indices. We summarize here all the mass predictions that are extracted from single currents and less than $4.5 \mathrm{GeV}$.

\begin{tabular}{cc|cc|cc}
\hline \hline Current & Structure & $s_{0}\left[\mathrm{GeV}^{2}\right]$ & Borel Mass $\left[\mathrm{GeV}^{2}\right]$ & Mass $[\mathrm{GeV}]$ & $(J, P)$ \\
\hline$\xi_{14}$ & {$\left[\Sigma_{c}^{+} \bar{D}^{0}\right]$} & $20-24$ & $4.12-4.52$ & $4.45_{-0.13}^{+0.17}$ & $(1 / 2,-)$ \\
$\psi_{2}$ & {$\left[\Sigma_{c}^{++} \bar{D}^{-}\right]$} & $19-23$ & $3.95-4.47$ & $4.33_{-0.13}^{+0.17}$ & $(1 / 2,-)$ \\
\hline$\xi_{33 \mu}$ & {$\left[\Sigma_{c}^{+} \bar{D}^{* 0}\right]$} & $20-24$ & $3.97-4.41$ & $4.46_{-0.13}^{+0.18}$ & $(3 / 2,-)$ \\
$\psi_{2 \mu}$ & {$\left[\Sigma_{c}^{*++} \bar{D}^{-}\right]$} & $20-24$ & $3.88-4.41$ & $4.45_{-0.13}^{+0.16}$ & $(3 / 2,-)$ \\
$\psi_{9 \mu}$ & {$\left[\Sigma_{c}^{++} \bar{D}^{*-}\right]$} & $19-23$ & $3.94-4.27$ & $4.37_{-0.13}^{+0.18}$ & $(3 / 2,-)$ \\
\hline$\xi_{13 \mu \nu}$ & {$\left[\Sigma_{c}^{*+} \bar{D}^{* 0}\right]$} & $20-24$ & $3.51-4.00$ & $4.50_{-0.12}^{+0.18}$ & $(5 / 2,-)$ \\
\hline \hline
\end{tabular}

\section{$7 \quad$ Three-body system}

There are various two-body interpretations of the exotic hadrons, as we have reviewed in previous sections. There are also some three-body interpretations for exotic hadrons. For example, combining the Faddeev equations with the chiral unitary model [531, 532], the authors interpreted the $Y(2175)$ as a dynamically generated state in the $\phi K \bar{K}$ system. In this section, we review some of these studies. We note that the three-body system have also been extensively studied within the chiral effective field theory $[533,534,535,536,537]$ and by using Lattice QCD $[538,539,540,541,542,543,544,545,546$, $547,548,549,550,551,552,540,553,554]$ in recent years, but we shall not review them in the present paper, and just recommend interested readers to the above references for detailed discussions.

\subsection{A short introduction to Faddeev equations}

When studying a three-body system, usually one does not consider the "direct" three-body interactions, as shown in Fig. 11. Instead, one needs to consider all the possible two-body exchanges/interactions in a system of three particles. One can use the Faddeev equations, named after their inventor Ludvig Faddeev [555]. We refer to Ref. [556] for detailed instructions.

In a three-body system there are three different two-body subsystems. The idea of the Faddeev equations is to sum up all the pair forces in each two-body subsystem to infinite order among all three particles. First we write the Schrödinger equation for a three-body system as

$$
\left(H_{0}+\sum_{i=1}^{3} V_{i}\right) \Psi=E \Psi
$$

where $H_{0}$ is the kinetic energy of the relative motion for three particles; $V_{1}=V_{23}, V_{2}=V_{31}$, and $V_{3}=V_{12}$ are the three interactions in the two-body subsystems. Then we transfer Eq. (62) into its integral form:

$$
\Psi=\frac{1}{E-H_{0}} \sum_{i=1}^{3} V_{i} \Psi \equiv G_{0} \sum_{i} V_{i} \Psi
$$




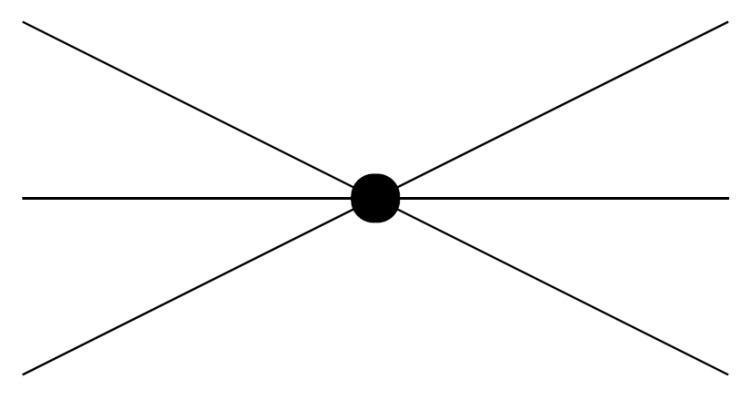

Figure 11: "Direct" three-body interactions.

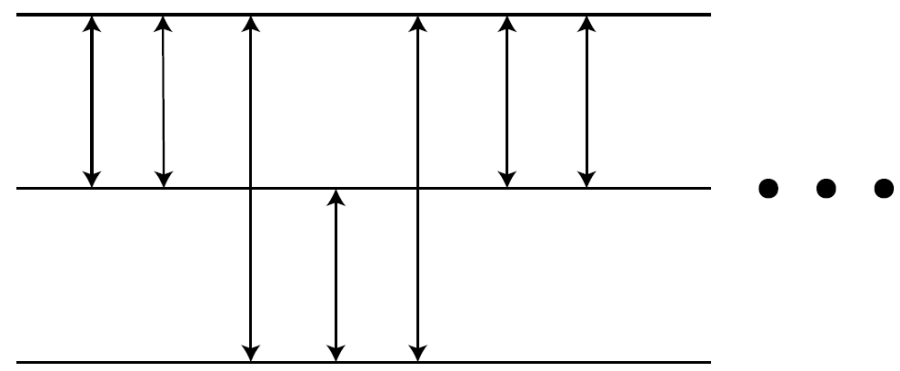

Figure 12: Diagrammatic representation of Eq. (64), where all the pair forces in each two-body subsystem have been summed up iteratedly to infinite order.

and further iterate it many times to be:

$$
\Psi=G_{0} \sum_{i} V_{i} G_{0} \sum_{j} V_{j} G_{0} \sum_{k} V_{k} \cdots \Psi .
$$

This can be shown graphically in Fig. 12, where all the pair forces in each two-body subsystem have been summed up iteratedly to infinite order.

To solve Eq. (64), we decompose $\Psi$ into three Faddeev components:

$$
\Psi=\sum_{i} \psi_{i}=\sum_{i} G_{0} V_{i} \Psi
$$

where $\psi_{i}=G_{0} V_{i} \Psi$ is that part of $\Psi$ having $V_{i}$ as the last interaction to the left. As an example, we show $\psi_{1}$ graphically in Fig. 13 .

We can further iterate $\psi_{i}$ to be:

$$
\psi_{i}=G_{0} V_{i} \Psi=G_{0} V_{i} \sum_{j} \psi_{j}=G_{0} V_{i} \psi_{i}+G_{0} V_{i} \sum_{j \neq i} \psi_{j},
$$

so that

$$
\psi_{i}=\left(\mathbf{1}-G_{0} V_{i}\right)^{-1} G_{0} V_{i} \sum_{j \neq i} \psi_{j}
$$




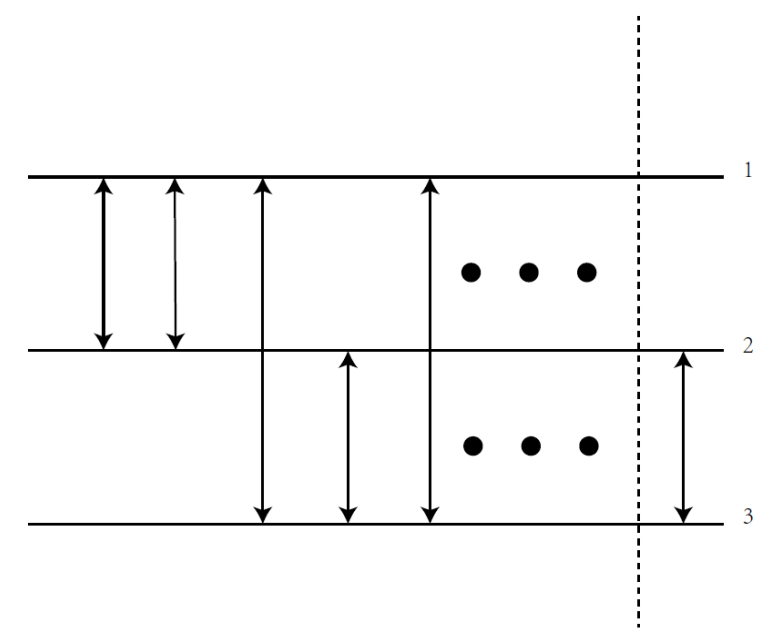

Figure 13: Diagrammatic representation of the Faddeev component $\psi_{1}$.

The kernel can be simplified to be

$$
\begin{aligned}
\left(\mathbf{1}-G_{0} V_{i}\right)^{-1} G_{0} V_{i} & =\left(\mathbf{1}+G_{0} V_{i}+G_{0} V_{i} G_{0} V_{i}+\cdots\right) G_{0} V_{i} \\
& =G_{0}\left(V_{i}+V_{i} G_{0} V_{i}+V_{i} G_{0} V_{i} G_{0} V_{i}+\cdots\right) \\
& \equiv G_{0} t_{i}
\end{aligned}
$$

where $t_{i}=\left(\mathbf{1}-V_{i} G_{0}\right)^{-1} V_{i}$ sums up $V_{i}$ to infinite order.

Finally, we arrive at three coupled equations

$$
\psi_{i}=G_{0} t_{i} \sum_{j \neq i} \psi_{j}
$$

which are called as the Faddeev equations. For identical particles, these equations can be simplified. Again, we refer Refs. [555, 556] for detailed discussions.

\subsection{Applications of Faddeev equations to exotic hadrons}

The Faddeev equations have been widely applied to study the quantum-mechanical three-body problem, such as three-nucleon bound states ( $N N N$ [557], $\Xi N N$ [558] and $\Omega N N$ [559]) and $\bar{K} N N$ quasi-bound state [560] in nuclear physics. Here we review its applications to exotic hadrons in hadron physics.

In Refs. [531, 532] the authors developed a method to study the meson-meson-baryon system by solving the Faddeev equations together with the chiral unitary model. Here we briefly review its application to the $Y(2175)$ [532]. There the $Y(2175)$ was interpreted as a $\phi f_{0}(980)$ resonant state, with the $f_{0}(980)$ dynamically generated in the $K \bar{K}$ channel. Hence, to study the $Y(2175)$, the authors solved the Faddeev equations in the three-body $\phi K \bar{K}$ system.

The full three-body scattering matrix is

$$
T=T^{1}+T^{2}+T^{3}
$$

where $T^{i}$ is the Faddeev partition. Note that $\Psi$ and $\psi_{i}$ are used in the previous subsection. They further write $T^{i}$ as

$$
T^{i}=t^{i} \delta^{3}\left(\vec{k}_{i}^{\prime}-\vec{k}_{i}\right)+T_{R}^{i j}+T_{R}^{i k}, \quad i \neq j \neq k=1,2,3,
$$




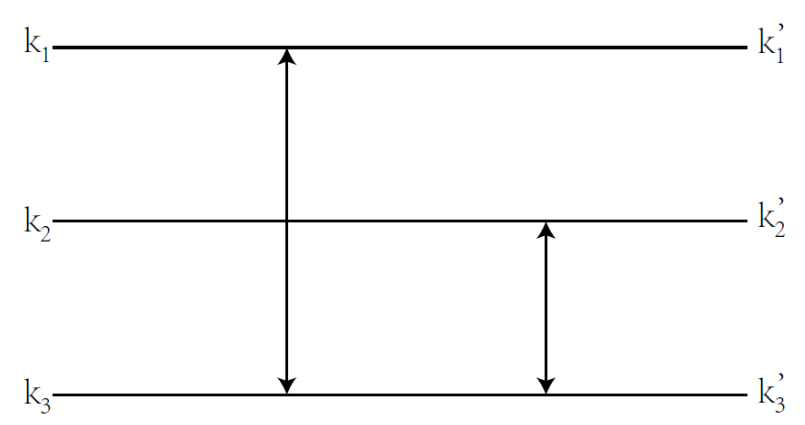

(a)

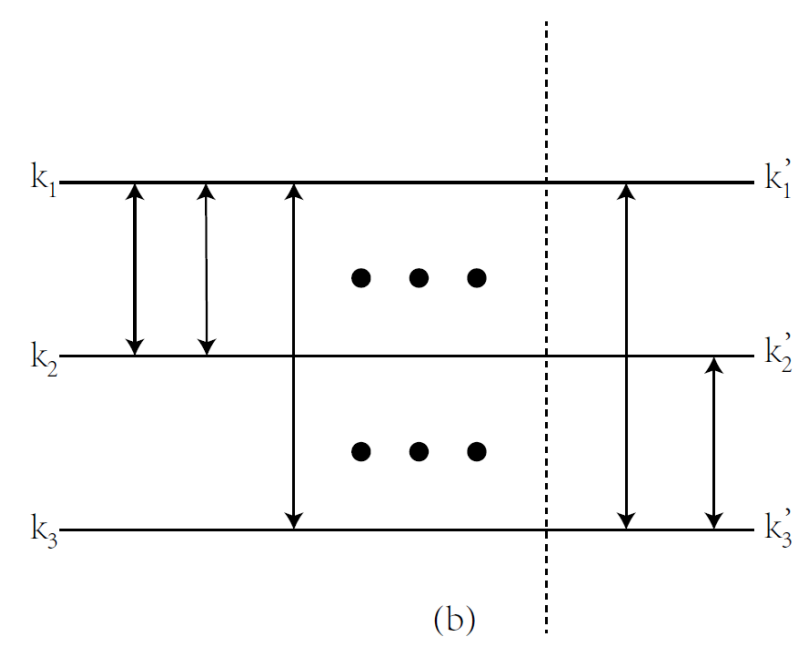

(b)

Figure 14: Diagrammatic representations of the terms (a) $t^{1} g^{12} t^{2}$ and (b) $T_{R}^{12}$.

where $t^{i}$ is the two-body scattering matrix where the particle $i$ is a spectator; the term $t^{i} \delta^{3}\left(\vec{k}_{i}^{\prime}-\vec{k}_{i}\right)$ corresponds to a "disconnected" diagram and should be removed; $T_{R}^{i j}$ sums up all the diagrams with the last two $t$ matrices being $t_{j}$ and $t_{i}$ :

$$
\begin{aligned}
T_{R}^{i j} & =t^{i} g^{i j} t^{j}+t^{i} g^{i j} t^{j} g^{j k} t^{k}+t^{i} g^{i j} t^{j} g^{j i} t^{i}+\cdots \\
& \rightarrow t^{i} g^{i j} t^{j}+t^{i}\left[G^{i j i} T_{R}^{j i}+G^{i j k} T_{R}^{j k}\right], \quad i \neq j \neq k=1,2,3 .
\end{aligned}
$$

In the above expression $g^{i j}$ is the three-body propagator:

$$
g^{i j}=\left(\prod_{r=1}^{D} \frac{1}{2 E_{r}}\right) \frac{1}{\sqrt{s}-E_{i}\left(\vec{k}_{i}\right)-E_{j}\left(\vec{k}_{j}\right)-E_{k}\left(\vec{k}_{i}+\vec{k}_{j}\right)+i \epsilon} .
$$

All the terms $t^{i} g^{i j} t^{j} g^{j k} t^{k}$ are written as $\left.t^{i} G^{i j k}\left(t^{j} g^{j k} t^{k}\right)\right|_{\text {on-shell }}$ :

$$
G^{i j k}=\int \frac{d^{3} k^{\prime \prime}}{(2 \pi)^{3}} \frac{N_{l}}{2 E_{l}} \frac{N_{m}}{2 E_{m}} \frac{F^{i j k}\left(\sqrt{s}, \vec{k}^{\prime \prime}\right)}{\sqrt{s_{l m}}-E_{l}\left(\vec{k}^{\prime \prime}\right)-E_{m}\left(\vec{k}^{\prime \prime}\right)+i \epsilon}, \quad i \neq j, j \neq k, i \neq l \neq m,
$$

where

$$
F^{i j k}=t^{j}\left(\sqrt{s_{\text {int }}}\left(\vec{k}^{\prime \prime}\right)\right)\left(\frac{g_{\text {off-shell }}^{j k}}{g_{\text {on-shell }}^{j k}}\right)\left[t^{j}\left(\sqrt{s_{\text {int }}}\left(\vec{k}_{j}^{\prime}\right)\right)\right]^{-1}
$$

The detailed explanations of the above equations can be found in Refs. [531, 532]. As examples, we show $t^{1} g^{12} t^{2}$ and $T_{R}^{12}$ graphically in Fig. 14 .

The two-body scattering matrices $t^{i}$ for the interactions of pseudoscalar and pseudoscalar mesons can be found in Ref. [561], while those for the interactions of pseudoscalar and vector mesons can be found in Ref. [562]. Using them as inputs, the authors solved the Faddeev equations, Eqs. (73), in the chiral unitary approach. They found a clear sharp peak of $\left|T_{R}\right|^{2} \equiv\left|T_{R}^{12}+T_{R}^{13}+T_{R}^{21}+T_{R}^{23}+T_{R}^{31}+T_{R}^{32}\right|^{2}$ around $2150 \mathrm{MeV}$ with a narrow width about tens of $\mathrm{MeV}$, as shown in Fig. 15. This structure can be associated to the $Y(2175)$, so their results indicate that the $Y(2175)$ is a dynamically generated resonant state in the $\phi K \bar{K}$ system. We refer to Ref. [532] for more discussions, where the coupled $\phi \pi \pi$ channel has also been taken into account. 


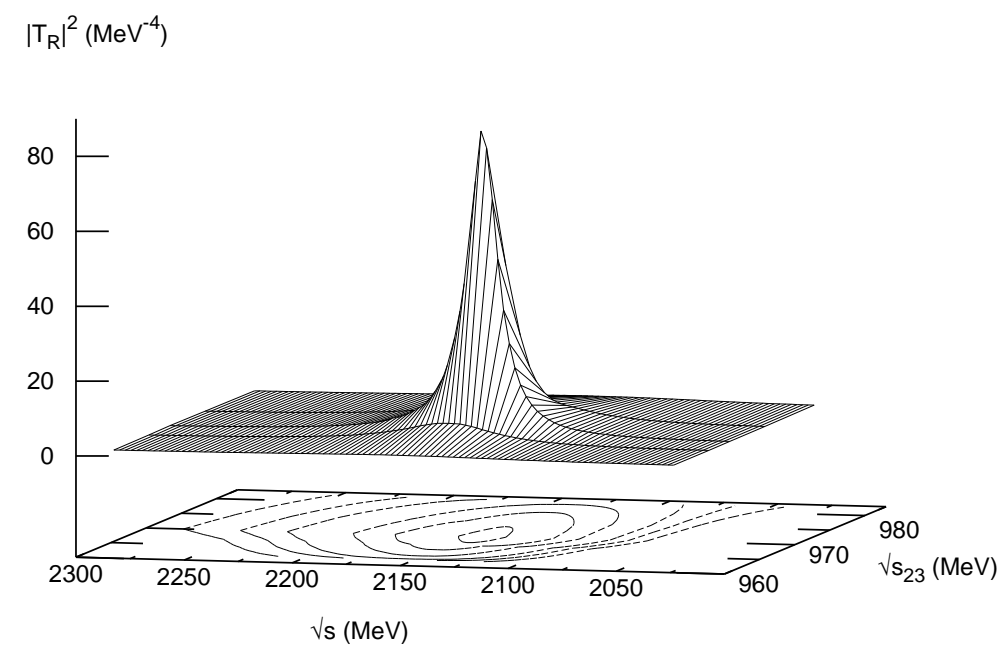

Figure 15: The $\phi K \bar{K}$ squared amplitude $\left|T_{R}\right|^{2} \equiv\left|T_{R}^{12}+T_{R}^{13}+T_{R}^{21}+T_{R}^{23}+T_{R}^{31}+T_{R}^{32}\right|^{2}$. The pole at $\sqrt{s} \sim 2150 \mathrm{MeV}$ is associated to the $Y(2175)$, and the pole at $\sqrt{s_{23}} \sim 970 \mathrm{MeV}$ is associated to the $f_{0}(980)$. Taken from Ref. [532].

The above method has been systematically applied to study the meson-meson-baryon (two pseudoscalar mesons and one octet baryon) system, and many dynamically generated resonant states are proposed. For examples, the $N^{*}(1710)$ is interpreted as a dynamically generated resonance in the $\pi \pi N$ system with $I=1 / 2$ and $J^{P}=1 / 2^{+}$[563]. The three mesons system has also been systematically studied within the same approach in Refs. [564, 565]. For examples, the $Y(4260)$ is interpreted as a dynamically generated resonance in the $J / \psi K \bar{K}$ system [564]; an exotic state with mass around 4140 $\mathrm{MeV}$ and $I=1 / 2$ was predicted in the $D D K$ system [565].

A similar approach was applied to study the $\bar{K} N \pi$ system by solving the Faddeev equations with relativistic kinematics in Ref. [566], and a $\bar{K} N \pi$ resonance with $I=1$ and $J^{P}=3 / 2^{-}$was predicted to be around $1570 \pm 10 \mathrm{MeV}$. The $P$-wave three-body $B^{*} B^{*} \bar{K}$ system is investigated in Ref. [567], and the results suggest that there exist $P$-wave $B^{*} B^{*} \bar{K}$ bound states with quantum numbers $J^{P}=0^{+} / 1^{+} / 2^{+}$, all of which locate at $30-40 \mathrm{MeV}$ below the $B^{*} B_{s 1}$ threshold. The $B^{(*)} B^{*} B^{*}$ systems are investigated in Ref. [568], and a unique bound state of three B mesons with $I=1 / 2$ and $J^{P}=2^{-}$was predicted to be around $90 \mathrm{MeV}$ below the threshold of three $B$-mesons.

\subsection{Fixed center approximation}

The Faddeev equations are sometimes not easy to be solved, so the fixed center approximation was proposed to deal with them $[569,570,571,572]$. In Ref. [573] this approach within the chiral unitary model was applied to study the $f_{2}(1270), \rho_{3}(1690), f_{4}(2050), \rho_{5}(2350)$ and $f_{6}(2510)$ resonances as multi- $\rho(770)$ states, and later on to many other exotic structures. In this paper we briefly review its application to the three-body $N \rho \rho$ system [574].

In Ref. [574] the authors investigated the three-body $N \rho \rho$ system by considering that two of the $\rho$ mesons are clusterized forming an strongly binding $f_{2}(1270)$ resonance, i.e., $N-(\rho \rho)_{f_{2}(1270)}$. In such a situation one can use the fixed center approximation to the Faddeev equations. We show this diagram- 


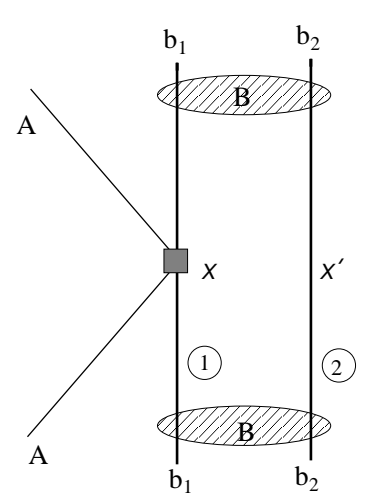

a)

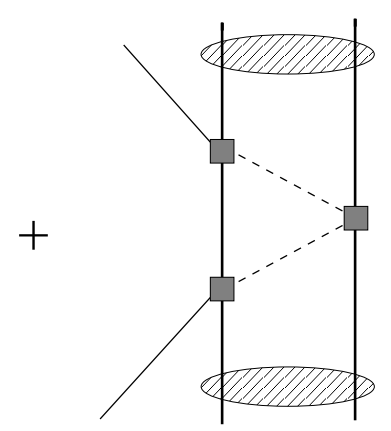

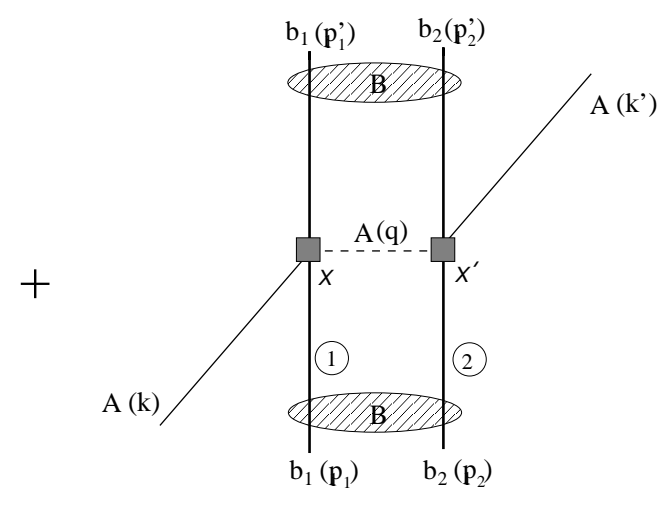

b)

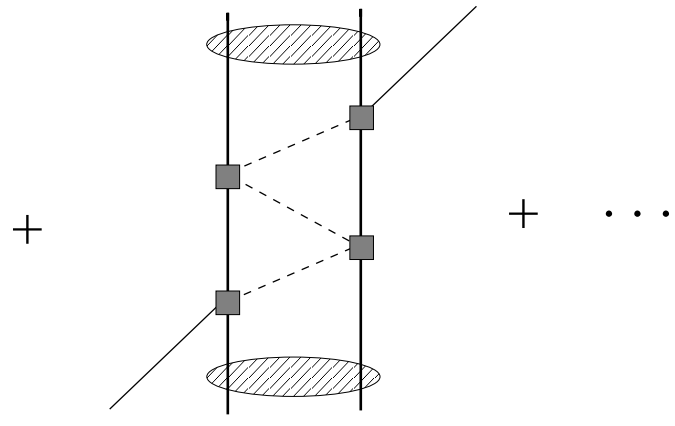

c)

Figure 16: Diagrammatic representation of the fixed center approximation to the Faddeev equations. $a), b$ ) and $c$ ) represent single, double, and multiple scattering contributions, respectively. Taken from Ref. [574].

matically in Fig. 16, where the external particle $N$ interacts successively with the other two $\rho$ mesons forming the $f_{2}(1270)$. The total scattering matrix is

$$
T=T_{1}+T_{2},
$$

where $T_{i}(i=1,2)$ are the two partition functions, accounting for all the diagrams starting with the interaction of $N$ and the $i$-th $\rho$ meson of the compound system:

$$
\begin{aligned}
& T_{1}=t_{1}+t_{1} G_{0} T_{2}, \\
& T_{2}=t_{2}+t_{2} G_{0} T_{1} .
\end{aligned}
$$

Here $t_{i}$ is the $N \rho$ scattering amplitude, which has been systematically investigated in Ref. [575] and the results therein can be used here as inputs; $G_{0}$ is the loop function for the particle $N$ propagating inside the compound system

$$
G_{0}(s)=\frac{1}{\sqrt{2 \omega_{f_{2}(1270)} 2 \omega_{f_{2}(1270)}^{\prime}}} \int \frac{d^{3} \vec{q}}{(2 \pi)^{3}} \times F_{f_{2}(1270)}(q) \frac{M_{N}}{E_{N}(\vec{q})} \frac{1}{q^{0}-E_{N}(\vec{q})+i \epsilon},
$$

where the form factor $F_{f_{2}(1270)}(q)$ is

$$
F_{f_{2}(1270)}(q)=\frac{1}{\mathcal{N}} \int_{p<\Lambda,|\vec{p}-\vec{q}|<\Lambda} d^{3} p \times \frac{1}{M_{f_{2}}-2 \omega_{\rho}(\vec{p})} \frac{1}{M_{f_{2}}-2 \omega_{\rho}(\vec{p}-\vec{q})},
$$



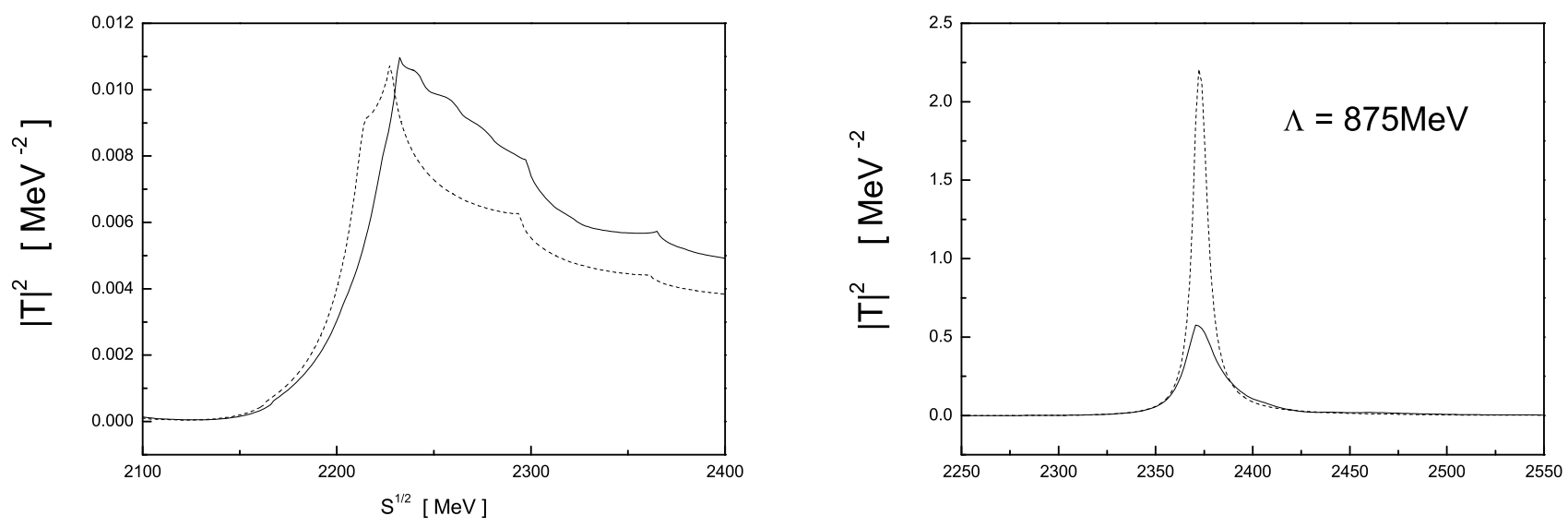

Figure 17: We show the unitarized $N \rho \rho$ squared amplitude $|T|^{2}$ in the left panel, and the unitarized $\Delta \rho \rho$ squared amplitude in the right panel. In both panels the solid and dashed lines denote the cases with and without the $f_{2}(1270)$ decay width, respectively. Taken from Ref. [574].

and the normalization factor $\mathcal{N}$ is

$$
\mathcal{N}=\int_{p<\Lambda} d^{3} p \frac{1}{\left(M_{f_{2}}-2 \omega_{\rho}(\vec{p})\right)^{2}} .
$$

We recommend interested readers to Refs. $[573,574]$ for detailed explanations of the above equations, where the wave function of the $f_{2}(1270)$ as well as its width are discussed.

Because there are two identical $\rho$ mesons, one has $T_{1}=T_{2}$, and Eqs. (76), (77) and (78) can be simplified to be:

$$
T=2 T_{1}=2 t_{1}+2 t_{1} G_{0} T_{1}=\frac{2 t_{1}}{1-G_{0} t_{1}} .
$$

The results are shown in the left panel of Fig. 17, where there is a peak at around $2227 \mathrm{MeV}$ with a width of $100 \mathrm{MeV}$. This peak does not have a standard Breit-Wigner form, so it could be due to the cusp effect.

In Ref. [574] the authors also applied the same approach to investigate the three-body $\Delta \rho \rho$ system. The results are shown in the right panel of Fig. 17, where there is a peak around $2372 \mathrm{MeV}$ with an approximate Breit-Wigner shape. This peak can be associated with the $\Delta(2390)$ of $J^{P}=7 / 2^{+}[576]$.

The fixed center approximation to the Faddeev equations has been systematically applied to study the $X$-multi- $\rho$ systems [577, 578]. For examples, in Ref. [577] the $K_{2}^{*}(1430), K_{3}^{*}(1780), K_{4}^{*}(2045)$ and $K_{5}^{*}(2380)$ are interpreted as molecules made of an increasing number of $\rho(770)$ and one $K^{*}(892)$ mesons; in Ref. [578] the $D^{*}$-multi- $\rho$ system was investigated and several charmed resonances, $D_{3}^{*}, D_{4}^{*}, D_{5}^{*}$ and $D_{6}^{*}$, were predicted to be around $2800-2850 \mathrm{MeV}, 3075-3200 \mathrm{MeV}, 3360-3375 \mathrm{MeV}$ and $3775 \mathrm{MeV}$, respectively. The above method has also been applied to study the $N K \bar{K}$ system [579], the $\eta K \bar{K}$ system [580], the $D K \bar{K}$ system [581], the $\bar{K} N N$ system [582], the $B D \bar{D}$ system [583], and the $K D \bar{D}^{*}$ system [584], etc.

\section{The Skyrme model and the chiral quark-soliton model}

The Skyrme model was proposed by T. H. R. Skyrme in 1961 by introducing the Skyrme term to the nonlinear sigma model [585]. Within this model the baryons appear as collective excitations of 
the meson fields. In fact, the non-trivial topological field configuration, which is called as soliton, is identified with the baryon. Later in 1979, E. Witten developed this model by arguing that baryons indeed emerge as solitons in the large $N_{C}$ generalization of QCD [586]. He also studied static properties of nucleons in the Skyrme model with two light flavors [587]. The Skyrme model was widely applied in hadron physics. Based on the Skyrme model one might qualitatively understand the EMC observation that the quark spin contribution to the total nucleon spin was unexpectedly small [588]. This feature is intensively studied in the Skyrme model with three light flavors in order to understand the nucleon's strangeness content [589].

In the large $N_{C}$-limit the chiral quark-soliton model ( $\left.\chi \mathrm{QSM}\right)[590,591,592,593]$. is quite similar to the Skyrme model, and sometimes they are taken as the same method/approach. In the Skyrme model one has integrated out the quark fields, and so directly operates with the action involving the pseudoscalar mesons without the quark fields. Actually, one can obtain the effective action of the Skyrme type from the one of the chiral quark-soliton model by a gradient expansion.

The Skyrme model and the chiral quark-soliton model with three light flavors can be naturally applied to study exotic baryons, which emerge as rotational excitations of nucleons [45]. In this paper we briefly review some of these applications, separately in the following subsections. We note that there are lots of investigations within this approach, and we refer interested readers to the lecture note by Diakonov [594], the one by Weigel [595], and the one by Ma and Harada [596] for detailed instructions.

\subsection{A short introduction to the Skyrme model}

The Lagrangian of the nonlinear sigma model is

$$
\mathcal{L}_{N L \sigma}=\frac{f_{\pi}^{2}}{4} \operatorname{Tr}\left(\partial_{\mu} U(x) \partial^{\mu} U^{\dagger}(x)\right)
$$

Here $f_{\pi}$ is the decay constant of the Nambu-Goldstone boson $\pi$, and the field $U(x)$ is defined as

$$
\begin{aligned}
U(x) \equiv \exp \left(i \frac{\tau_{i} \phi_{i}}{f_{\pi}}\right) & =\cos \left(\frac{\tau_{i} \phi_{i}}{f_{\pi}}\right)+i \sin \left(\frac{\tau_{i} \phi_{i}}{f_{\pi}}\right) \\
& =\frac{1}{f_{\pi}}\left(\sigma+i \tau_{i} \pi_{i}\right)=\frac{M}{f_{\pi}}
\end{aligned}
$$

where $M \equiv \sigma+i \tau_{i} \pi_{i}$ is the meson field, and the field variables $\phi_{i}$ are related to $\pi_{i}$ through

$$
\frac{\tau_{i} \pi_{i}}{f_{\pi}}=\sin \left(\frac{\tau_{i} \phi_{i}}{f_{\pi}}\right) .
$$

Under the chiral transformation, the field $U(x)$ transforms as $U(x) \rightarrow g_{L} U(x) g_{R}^{\dagger}$, so the Lagrangian (83) is chirally invariant. With the nonlinear realization of the chiral symmetry, the Hamiltonian density of the nonlinear sigma model can be obtained as

$$
\mathcal{H}_{N L \sigma}=\frac{f_{\pi}^{2}}{4} \operatorname{Tr}\left(\partial_{0} U^{\dagger} \partial_{0} U\right)+\frac{f_{\pi}^{2}}{4} \operatorname{Tr}\left(\partial_{i} U(x) \partial_{i} U^{\dagger}(x)\right)
$$

and its energy can be expressed as

$$
\begin{aligned}
E_{N L \sigma} & =\frac{f_{\pi}^{2}}{4} \int d^{3} x \operatorname{Tr}\left(\partial_{0} U^{\dagger} \partial_{0} U\right)+\frac{f_{\pi}^{2}}{4} \int d^{3} x \operatorname{Tr}\left(\partial_{i} U(x) \partial_{i} U^{\dagger}(x)\right) \\
& \equiv E_{N L \sigma}^{\text {rotation }}+E_{N L \sigma}^{\text {static }} .
\end{aligned}
$$


Here the first term $E_{N L \sigma}^{\text {rotation }}$ is the rotational energy, and the second term $E_{N L \sigma}^{\text {static }}$ is the static energy. The static energy $E_{N L \sigma}^{\text {static }}$ is not stable: under the rescaling of the space coordinates $U(\mathbf{x}) \rightarrow U(\lambda \mathbf{x})$, one has $E_{N L \sigma}^{\text {static }} \rightarrow E_{N L \sigma}^{\text {static }} / \lambda$.

To avoid the above stability problem of the static energy, Skyrme introduced the so-called Skyrme term, and extended the nonlinear sigma model Lagrangian into the Skyrme model:

$$
\mathcal{L}_{\text {Skyrme }}=\frac{f_{\pi}^{2}}{4} \operatorname{Tr}\left(\partial_{\mu} U \partial^{\mu} U^{\dagger}\right)+\frac{1}{32 e^{2}} \operatorname{Tr}\left\{\left[U^{\dagger} \partial_{\mu} U, U^{\dagger} \partial_{\nu} U\right]\left[U^{\dagger} \partial^{\mu} U, U^{\dagger} \partial^{\nu} U\right]\right\}
$$

where $e$ is a dimensionless parameter, indicating the magnitude of the soliton. Its energy can be obtained as

$$
\begin{aligned}
E_{\text {Skyrme }} & =-\int d^{3} x \operatorname{Tr}\left[\frac{f_{\pi}^{2}}{4} L_{0} L_{0}+\frac{1}{16 e^{2}}\left[L_{0}, L_{i}\right]^{2}\right]-\int d^{3} x \operatorname{Tr}\left[\frac{f_{\pi}^{2}}{4} L_{i} L_{i}+\frac{1}{32 e^{2}}\left[L_{i}, L_{j}\right]^{2}\right] \\
& \equiv E_{\text {Skyrme }}^{\text {rotation }}+E_{\text {Skyrme }}^{\text {static }} .
\end{aligned}
$$

One can solve the following extremum stable condition

$$
\left.\frac{d E_{\text {Skyrme }}^{\text {static }}(\lambda)}{d \lambda}\right|_{\lambda=1}=0
$$

and the obtained stabilized solutions are called as Skyrme solitons or skyrmions.

Based on Eq. (88), the Witten-Wess-Zumino term was added at the level of the action to reproduce QCD's low energy anomalous structure [586, 597, 598]:

$$
\Gamma_{\text {Skyrme }}^{\mathrm{WZ}}=-\frac{i N_{c}}{240 \pi^{2}} \int d^{5} x \epsilon^{\mu \nu \rho \sigma \tau} \operatorname{Tr}\left[L_{\mu} L_{\nu} L_{\rho} L_{\sigma} L_{\tau}\right]
$$

where $N_{c}=3$ is the number of colors. Sometimes the symmetry breaking terms are also necessary:

$$
\begin{aligned}
\mathcal{L}_{\text {Skyrme }}^{\mathrm{SB}}= & \frac{f_{\pi}^{2} m_{\pi}^{2}+2 f_{K}^{2} m_{K}^{2}}{12} \operatorname{Tr}\left[U+U^{\dagger}-2\right]+\sqrt{3} \times \frac{f_{\pi}^{2} m_{\pi}^{2}-f_{K}^{2} m_{K}^{2}}{6} \operatorname{Tr}\left[\lambda_{8}\left(U+U^{\dagger}\right)\right] \\
& +\frac{f_{K}^{2}-f_{\pi}^{2}}{12} \operatorname{Tr}\left[\left(1-\sqrt{3} \lambda_{8}\right)\left(U\left(\partial_{\mu} U\right)^{\dagger} \partial^{\mu} U+U^{\dagger} \partial_{\mu} U\left(\partial^{\mu} U\right)^{\dagger}\right)\right] .
\end{aligned}
$$

The Skyrme model can be used to derive a series of states [599, 600, 601, 602]. In the three-flavor case, the lowest states are the $(\mathbf{8}, 1 / 2)$ and $(\mathbf{1 0}, 3 / 2)$, which can be used to describe the ground-state octet baryons of $J^{P}=1 / 2^{+}$and decuplet baryons of $J^{P}=3 / 2^{+}$. The remaining states are all exotic baryons, and the states at the next level are the $(\overline{\mathbf{1 0}}, 1 / 2),(\mathbf{2 7}, 1 / 2)$ and $(\mathbf{2 7}, 3 / 2)$.

In the chiral symmetry limit one can write the effective Hamiltonian as:

$$
\mathcal{H}_{\text {Skyrme }}=M_{c l}+\frac{1}{2 I_{1}} J(J+1)+\frac{1}{2 I_{2}}\left(C_{2}(\mathcal{R})-J(J+1)-\frac{N_{c}^{2}}{12}\right)+\mathcal{H}_{\text {Skyrme }}^{\prime}
$$

where $J$ denotes the baryon spin, $C_{2}(\mathcal{R})$ denotes the Casimir operator for the $S U(3)$ representation $\mathcal{R}$, and the other three parameters $M_{c l}$ and $I_{1,2}$ are treated as free parameters. In the Skyrme model the symmetry breaking Hamiltonian is

$$
\mathcal{H}_{\text {Skyrme }}^{\prime}=-\alpha D_{88}^{(8)}(A)
$$

which leads to both the first order correction to the baryon mass

$$
M_{B(\mathcal{R})}^{(1)}=-\alpha \delta_{B(\mathcal{R})}^{\mathcal{R}},
$$




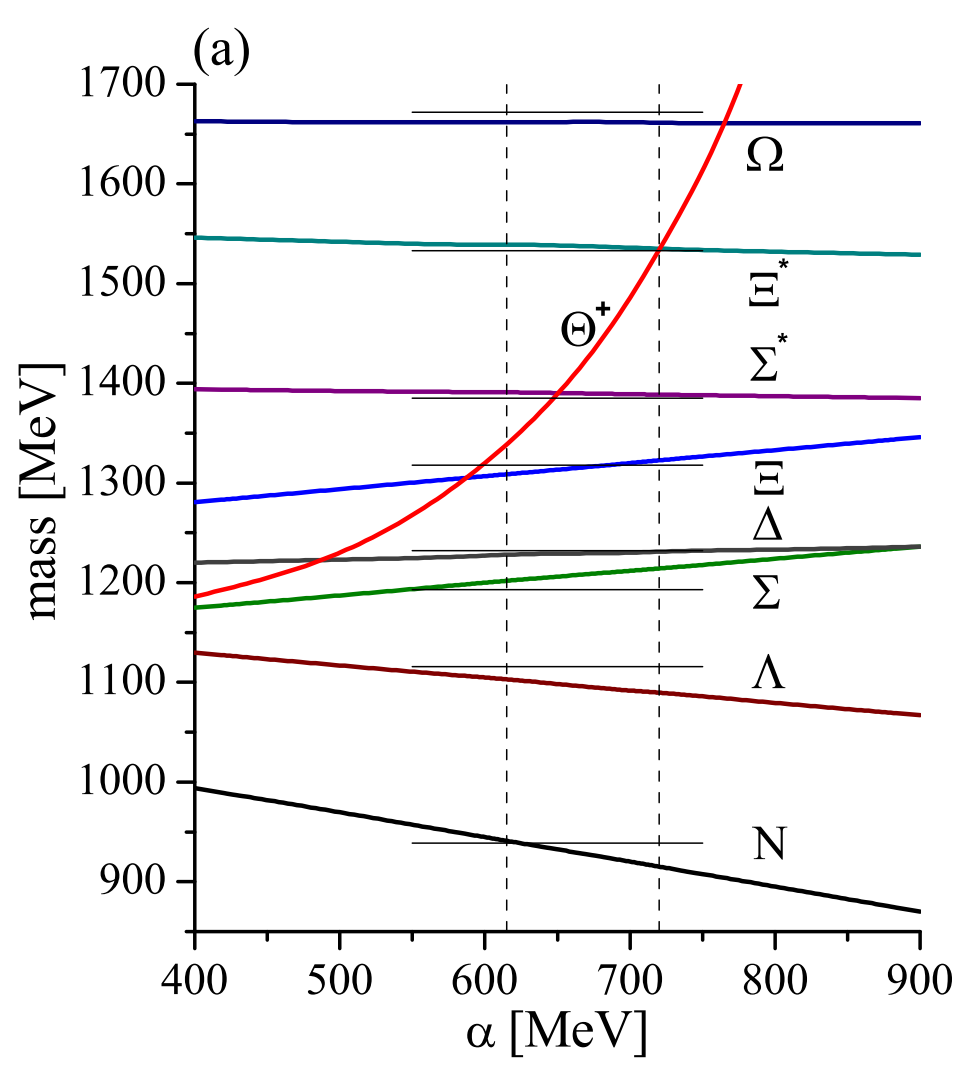

Figure 18: Masses of the ordinary baryons together with the mass of the $\Theta^{+}$, as functions of the parameter $\alpha$. Taken from Ref. [604].

and the second order correction

$$
M_{B(\mathcal{R})}^{(2)}=-2 I_{2} \alpha^{2} \sum_{\mathcal{R}^{\prime} \neq \mathcal{R}} \frac{\left(\delta_{B(\mathcal{R})}^{\mathcal{R}^{\prime}}\right)^{2}}{C_{2}\left(\mathcal{R}^{\prime}\right)-C_{2}(\mathcal{R})},
$$

We refer interested readers to Refs. [603, 604] for detailed discussions on the above equations. Especially, the mass of the $u u d d \bar{s}$ pentaquark belonging to $(\overline{\mathbf{1 0}}, 1 / 2)$ was predicted to be $[603,604]$

$$
M_{\Theta^{+}}=M_{8}+\frac{3}{2} \frac{\alpha^{2}}{\epsilon}-\frac{2}{8} \alpha-\frac{3}{112} \epsilon \approx 1530 \mathrm{MeV} .
$$

However, this mass value is quite sensitive to the choice of parameters. For example, the authors of Ref. [604] plot in Fig. 18 the results of the constrained fits, and one can find a rather steep rise of $M_{\Theta^{+}}$with $\alpha$. The above mass value $1530 \mathrm{MeV}$ is very close to the mass of the $\Theta^{+}$observed by LEPS [46], whose observation quickly led to renewed interest in the Skyrme model descriptions of baryons. Unfortunately, the $\Theta^{+}$was not confirmed in a series of high precise particle experiments [48].

The Skyrme model has many advantages in describing hadron physics, and we refer to Refs. [605, 595, 596] for detailed discussions. Especially, the Skyrme model can be applied to study both baryon and meson physics in free space [587], while it can also be applied to study nuclear matter and the medium modified hadron properties [606, 607, 608]. In recent years, the Skyrme model is extended so that a) the effects of the higher resonances and higher chiral order terms can be self-consistently analysed [609, 610,611, 612], and b) properties of heavy baryons containing a heavy quark can be systematically investigated [613, 614, 615, 616, 617]. 


\subsection{A short introduction to the chiral quark-soliton model}

In the previous subsection we have briefly introduced the Skyrme model. In this subsection we shall briefly introduce the chiral quark-soliton model $[618,619]$. Both of them have similar group structure, and can be applied to study the $\Theta^{+}$pentaquark belonging to the exotic $(\overline{\mathbf{1 0}}, 1 / 2)$ flavor representation. In fact, the prediction of the narrow width of the $\Theta^{+}$in the chiral quark-soliton model [45] stimulated the experimental search by LEPS [46]. We refer to Refs. [594, 620] for detailed discussions.

According to the chiral symmetry, the interaction of pseudoscalar mesons with constituent quarks can be written as:

$$
\mathcal{L}_{\text {eff }}=\bar{q}\left[i \partial^{\mu} \gamma_{\mu}-M \exp \left(i \gamma_{5} \pi^{A} \lambda^{A} / F_{\pi}\right)\right] q,
$$

where the $\pi^{A}$ fields are the ordinary pseudoscalar mesons $\pi, K$, and $\eta$. In the large $N_{c}$ limit baryons emerge as solitons of this chiral action [586]. The model of baryons based on the large $N_{c}$ limit is called the chiral quark-soliton model [620].

Actually, we can also use the word "soliton" for the self-consistent pion mean field in the nucleon. Because these Hartree-Fock states composed of valence- and sea-quarks are degenerate with respect to rotations in both space and flavor-space, we can use the following collective Hamiltonian to describe them in the rigid rotor approximation [621, 622]:

$$
\mathcal{H}_{\chi \mathrm{QSM}}=M_{c l}+\frac{J(J+1)}{2 I_{1}}+\frac{1}{2 I_{2}}\left(\mathcal{C}_{2}(\mathcal{R})-J(J+1)-\frac{N_{c}^{2}}{12}\right)+H_{\chi \mathrm{QSM}}^{\prime},
$$

where $M_{c l}$ is the classical mean-field energy of the quark system, $J$ is the baryon spin, and $C_{2}(\mathcal{R})$ is the Casimir operator for the $S U(3)$ representation $\mathcal{R}$. This Hamiltonian is very similar to the one of the Skyrme model given in Eq. (93) except a different symmetry breaking term, i.e.,

$$
H_{\chi \mathrm{QSM}}^{\prime}=\alpha D_{88}^{(8)}+\beta Y+\frac{\gamma}{\sqrt{3}} D_{8 i}^{(8)} J_{i}
$$

where $\alpha, \beta$, and $\gamma$ are parameters of order $\mathcal{O}\left(m_{s}\right), D_{a b}^{(\mathcal{R})}$ are $S U(3)$ Wigner rotation matrices, and $J_{i}$ are collective spin operators.

The symmetry-breaking term $H_{\chi \mathrm{QSM}}^{\prime}$ mixes different $S U(3)$ representations, so the collective wave functions can be written as the following linear combinations:

$$
\begin{aligned}
\left|B_{\mathbf{8}}\right\rangle= & \left|\mathbf{8}_{1 / 2}, B\right\rangle+c_{10}^{B}\left|\overline{\mathbf{1 0}}_{1 / 2}, B\right\rangle+c_{27}^{B}\left|\mathbf{2 7}_{1 / 2}, B\right\rangle \\
\left|B_{10}\right\rangle= & \left|\mathbf{1 0}_{3 / 2}, B\right\rangle+a_{27}^{B}\left|\mathbf{2 7}_{3 / 2}, B\right\rangle+a_{35}^{B}\left|\mathbf{3 5}_{3 / 2}, B\right\rangle \\
\left|B_{\overline{\mathbf{1 0}}}\right\rangle= & \left|\overline{\mathbf{1 0}}_{1 / 2}, B\right\rangle+d_{8}^{B}\left|\mathbf{8}_{1 / 2}, B\right\rangle+d_{27}^{B}\left|\mathbf{2 7}_{1 / 2}, B\right\rangle+d_{35}^{B}\left|\overline{\mathbf{3 5}}_{1 / 2}, B\right\rangle, \\
& \cdots
\end{aligned}
$$

where $\left|B_{\mathcal{R}}\right\rangle$ are the states which reduce to the $S U(3)$ representation $\mathcal{R}$ when $m_{s} \rightarrow 0$ and the spin index $J_{3}$ is suppressed. $c_{\mathcal{R}}^{B}, a_{\mathcal{R}}^{B}$, and $d_{\mathcal{R}}^{B}$ are the $m_{s}$-dependent coefficients. For example, we show in Fig. 19 the weight diagrams for the lowest-lying baryon multiplets, which contains the baryons with the hypercharge $Y^{\prime}=1$.

Assuming the state $N^{*}(1710)$ to be a member of the $(\overline{\mathbf{1 0}}, 1 / 2)$ flavor representation, Diakonov, Petrov and Polyakov investigated an exotic $Z^{+}$baryon with spin $1 / 2$, isospin 0 and strangeness +1 , and predicted its mass to be about $1530 \mathrm{MeV}$ and its total width to be less than $15 \mathrm{MeV}$ [45]. Now this state was known as the $\Theta^{+}$, which was first observed by LEPS [46]. However, we note that this small width actually resulted from an arithmetic error, as explained by Jaffe in Ref. [624], and correcting this error gives a width twice as large at least. See Ref. [589] for detailed discussions. 


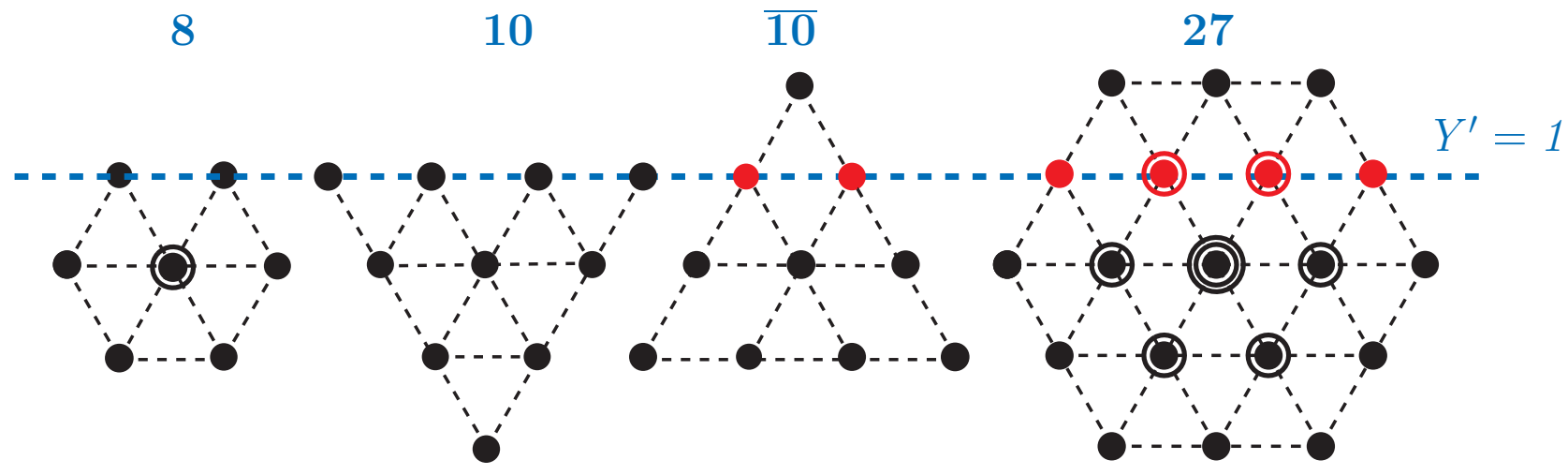

Figure 19: Weight diagrams for the lowest-lying baryon multiplets, including the flavor representations $\mathbf{8}, \mathbf{1 0}, \overline{\mathbf{1 0}}$, and 27. Taken from Ref. [623].

Later in Refs. [625, 626] the masses of the lowest-lying baryons were calculated by including the effects of isospin symmetry breaking arising from both the quark masses and electromagnetic selfenergies. The authors used the following collective Hamiltonian in the $S U(3)$ chiral soliton model [621, 622]:

$$
\mathcal{H}=M_{c l}+\mathcal{H}_{\text {rot }}+\mathcal{H}_{S B}
$$

where $\mathcal{H}_{\text {rot }}$ is the $1 / N_{c}$ rotational energy, and $\mathcal{H}_{S B}$ are the symmetry-breaking terms including both isospin and $S U(3)$ flavor symmetry breaking effects:

$$
\begin{aligned}
\mathcal{H}_{\text {rot }}= & \frac{1}{2 I_{1}} \sum_{i=1}^{3} J_{i}^{2}+\frac{1}{2 I_{2}} \sum_{p=4}^{7} J_{p}^{2}, \\
\mathcal{H}_{S B}= & \left(m_{d}-m_{u}\right)\left(\frac{\sqrt{3}}{2} \alpha D_{38}^{(8)}(\mathcal{R})+\beta T_{3}+\frac{\gamma}{2} \sum_{i=1}^{3} D_{3 i}^{(8)}(\mathcal{R}) J_{i}\right) \\
& +\left(m_{s}-\frac{m_{u}+m_{d}}{2}\right)\left(\alpha D_{88}^{(8)}(\mathcal{R})+\beta Y+\frac{\gamma}{\sqrt{3}} \sum_{i=1}^{3} D_{8 i}^{(8)}(\mathcal{R}) J_{i}\right) \\
& +\left(m_{u}+m_{d}+m_{s}\right) \sigma .
\end{aligned}
$$

We refer interested readers to Refs. [625, 626] for detailed discussions on the above equations.

The baryon mass splittings due to the isospin and $S U(3)$ flavor symmetry breaking up to the first order were investigated in Ref. [625], and those due to the second order flavor symmetry breaking were investigated in Ref. [626]. The authors estimated the mass of the $N^{*}$ as a member of the baryon antidecuplet to be $1687 \mathrm{MeV}$, which can be used to explain the $N^{*}(1685)$, a narrow bump-like structure observed by GRAAL in the $\gamma n \rightarrow \eta n$ quasi-free cross section in 2006 [627]. The authors also estimated the pion-nucleon sigma term to be $\sigma_{\pi N}=(50.5 \pm 5.4) \mathrm{MeV}$.

In 2015, another narrow resonant structure was observed by the GRAAL Collaboration in real Compton scattering off the proton. Its mass and width were determined to be $M=1.726 \pm 0.002 \pm$ $0.005 \mathrm{GeV}$ and $\Gamma=21 \pm 7 \mathrm{MeV}$, respectively. In the chiral quark-soliton model, this structure can be identified as a member of the eikosiheptaplet (27) with spin 3/2 [628]. Later in Ref. [623] the authors investigated the strong and radiative decay widths of the narrow nucleon resonances $N^{*}(1685)$ and $N^{*}(1726)$ together within the $S U(3)$ chiral quark-soliton model [623]. Especially, they found $\Gamma_{p^{*}(1726) \rightarrow p \gamma} / \Gamma_{n^{*}(1726) \rightarrow n \gamma}=3.76 \pm 0.64$, indicating that the production of $N^{*}(1726)$ is more likely to be observed in the proton channel. 


\section{Progresses from Lattice QCD}

Lattice QCD is the unique non-perturbative theoretical framework to study the hadron spectroscopy starting from the first principle QCD Lagrangian. In a lattice QCD simulation, the hadron mass $E_{n}$ can be extracted from the $N \times N$ time-dependence correlation function

$$
\begin{aligned}
C_{i j}(t) & =\left\langle\Omega\left|\mathcal{O}_{i}(t) \mathcal{O}_{j}^{\dagger}(0)\right| \Omega\right\rangle \\
& =\sum_{n=1}^{N} e^{-E_{n} t}\left\langle\Omega\left|\mathcal{O}_{i}\right| n\right\rangle\left\langle n\left|\mathcal{O}_{j}^{\dagger}(0)\right| \Omega\right\rangle
\end{aligned}
$$

where the interpolating operator $\mathcal{O}_{j}^{\dagger}(0)(j=1,2, \cdots, N)$ creates the state of interest from the vacuum $|\Omega\rangle$ at time $t=0$ and $\mathcal{O}_{i}(t)$ annihilates the state at a later Euclidean time $t . E_{n}$ is the eigenvalue of the Hamiltonian for the system. These creation and annihilation operators should have the same $J^{P C}$ quantum numbers, which are called interpolators. In principle they can couple to all physical eigenstates $|n\rangle$ with the same given quantum numbers but different magnitudes of overlaps

$$
\left\langle n\left|\mathcal{O}_{i}^{\dagger}\right| \Omega\right\rangle \equiv Z_{i}^{n}
$$

In lattice QCD, the correlation functions are usually evaluated by using a large basis of interpolators constructing of quark and gluon fields, the Dirac gamma matrices and the gauge-covariant derivative operator. To search for the tetraquark and pentaquark signatures, the two-particle interpolators are usually adopted to deduce the two-point correlation functions. If the interpolator basis is complete enough, one can extract and identify all two-particle discrete energy levels with the given quantum numbers from the full correlation functions. The exotic multiquark states are related to the extra energy levels in addition to the expected non-interacting two-particle states. The two-particle energies are obtained from the correlation function by solving the generalized eigenvalue problem (GEVP) [629, 630]:

$$
C_{i j}(t) v_{j}^{n}\left(t, t_{0}\right)=\lambda_{n}\left(t, t_{0}\right) C_{i j}\left(t_{0}\right) v_{j}^{n}\left(t, t_{0}\right)
$$

where $v_{j}^{n}\left(t, t_{0}\right)$ is an eigenvector and $t_{0}$ a suitably chosen reference time $t_{0}<t$. The eigenvalues feature the relevant state energies [629]

$$
\lambda_{n}\left(t, t_{0}\right) \approx A_{n} e^{-E_{n}\left(t-t_{0}\right)}+\cdots
$$

Fitting this exponential decay behavior of the time dependence for each of these quantities, the exact discrete spectrum $E_{n}$ can be extracted from the effective mass plateau of the eigenvalue $\lambda_{n}[631,632$, $633,634,635]$

$$
E_{n}^{e f f}(t)=\ln \frac{\lambda_{n}(t)}{\lambda_{n}(t+1)} .
$$

In the infinite volume, a bound state of two particles can be defined as a discrete energy eigenstate of the Hamiltonian with energy level below the two-particle threshold within the Lüscher's method [636, 637, 629, 638, 639]. In a two-particle scattering process, the resonant character on the lattice can be observed as the negative center of mass (c.m.) momentum $q^{2}$, which corresponds to the attractive interaction between the two particles and renders the energy level lower than the noninteracting threshold. The Lüscher's formula provides a direct relation of $q^{2}$ and the elastic scattering phase shift $\delta(q)$ in the infinite volume, e. g. in the case of the $s$-wave elastic scattering

$$
q \cot \delta(q)=\frac{1}{\pi^{3} / 2} \mathcal{Z}_{00}\left(1 ; q^{2}\right),
$$


where $\mathcal{Z}_{00}\left(1 ; q^{2}\right)$ is the zeta-function. In the limit of $q^{2} \rightarrow-\infty$, the phase shift will approximate $\cot \delta(q) \approx-1$ if there exists a true bound state at the particular energy in the infinite volume. Therefore, one usually needs to study the quantities $q^{2}$ and $\cot \delta(q)$ to really identify a bound state in a lattice simulation. We refer to a recent review article [640] for detailed discussion, and refer to Refs. [641, 642] from the viewpoints of the chiral perturbation theory.

In the last decade, there are some remarkable progresses in the study of new hadron states by lattice QCD. In this section, we try to introduce these investigations, such as the studies on the $Y(4260)$, $X(3872)$, the charged $Z_{c}$ states, the doubly heavy tetraquark states and the hidden-charm pentaquark states.

\section{$9.1 Y(4260)$}

The underlying structure of the $Y(4260)$ meson was studied by TWQCD Collaboration [643]. They calculated the mass spectra of the hidden-charm hybrid mesons $(\bar{c} g c)$, molecules and diquark-antidiquark tetraquarks with $J^{P C}=1^{--}$, in quenched lattice QCD with exact chiral symmetry. The authors computed the time correlation functions for all operators in the above various schemes and detected all these exotic resonances. However, the lowest-lying hybrid charmonium lies about $250 \mathrm{MeV}$ above the mass of $Y(4260)$. In contrast, the $1^{--}$hidden-charm molecule $(\bar{u} c \bar{c} u)$ and tetraquark $(u c \bar{u} \bar{c})$ states were detected with masses 4238(31)(57) MeV and 4267(68)(83) MeV respectively, which are in good agreement with the mass of $Y(4260)$. Finally, the authors suggested the $Y(4260)$ to be a $D_{1} \bar{D}$ molecule since it has a better overlap with the molecular operator than any other ones. Besides, they also detected resonances of molecular and tetraquark states with quark contents $\operatorname{cs} \bar{c} \bar{s}$ around $4450 \pm 100$ $\mathrm{MeV}$, and with quark contents $c c \bar{c} \bar{c}$ around $6400 \pm 50 \mathrm{MeV}$.

Before the observation of $Y(4260)$, the mass spectrum of the charmonium hybrids were predicted by the UKQCD quenched lattice NRQCD [644, 645, 646] and anisotropic lattice simulations [647]. In Ref. [648], the Hadron Spectrum Collaboration performed dynamical lattice QCD calculations to study the mass spectra of highly excited charmonium and charmonium hybrid mesons. To provide a large basis of operators with a range of spatial structures in each channel, the authors used the derivative-based construction for operators of the general form $\bar{\psi} \Gamma \stackrel{\leftrightarrow}{D_{i}} \stackrel{\leftrightarrow}{D}_{j} \cdots \psi$ to calculate the two-point correlation functions. Their computations were presented for up and down quarks corresponding to a pion mass of $400 \mathrm{MeV}$ at a single lattice spacing. They calculated the dynamical spectrum of charmonium hybrids with exhaustive quantum numbers including the exotic ones $\left(0^{+-}, 1^{-+}, 2^{+-}\right)$up to $4.5 \mathrm{GeV}$. Their results identified the scheme that the hybrid meson can be interpreted as a colour-octet quark-antiquark pair coupled to a $J_{g}^{P_{g} C_{g}}=1^{+-}$chromomagnetic gluonic excitation. In this scheme, the lightest hybrid supermultiplet consists of negative-parity states $J^{P C}=(0,1,2)^{-+}, 1^{--}$, in which the colour-octet quarkantiquark pair is in S-wave. Such a pattern suggested that the lightest gluonic excitation has an energy scale of 1.2-1.3 GeV, compared with the conventional charmonium bound states. The first excited hybrid supermultiplet composed of a P-wave colour-octet quark-antiquark pair coupled to an excited gluonic field would contain positive-parity states with $J^{P C}=0^{+-},\left(1^{+-}\right)^{3},\left(2^{+-}\right)^{2}, 3^{+-},(0,1,2)^{++}$. The vector hybrid charmonium with $J^{P C}=1^{--}$was extracted at $M-M_{\eta_{c}} \approx 1.3 \mathrm{GeV}$, which agrees well with the mass of $Y(4260)$ and supports the hybrid interpretation of this state. The charmonium hybrid interpretation for $Y(4260)$ was also performed in Ref. [649], in which the authors calculated the masses of the ground and first excited states for the $\bar{c} g c$ hybrid mesons with $J^{P C}=0^{-+}, 1^{--}$and $1^{++}$. They finally concluded that the $Y(4260)$ meson can not be identified as the ground state of the $1^{--}$hybrid, but most probably the first excited state of the $1^{--}$hybrid charmonium state. However, the QCD sum rule calculation gave much lower mass prediction for the vector hybrid charmonium, which was around $3.4 \mathrm{GeV}$ [650]. The heavy quarkonium hybrid states with various flavor structures and quantum numbers have been extensively studied in Refs. [650, 651, 495].

Since the lattice investigations are always performed with unphysical-heavy light quarks, it is sig- 
nificant to explore the changes in the pattern of states with light-quark masses closer to their physical values [652]. For the charmonium systems, the light-quark mass dependence enters through the sea quark content in the dynamical gauge field ensembles. In the case of the open-charm charmed mesons, the existence of a valence light quark affects the spectrum directly. To investigate the light quark dependence effect on lattice QCD simulation, the Hadron Spectrum Collaboration presented spectra of the excited charmonium, open-charm $D$ and $D_{s}$ mesons from dynamical lattice QCD calculations with a pion mass $m_{\pi} \sim 240 \mathrm{MeV}$ in Ref. [653]. The charmonium spectra labelled by $J^{P C}$ were shown in Fig. 20 together with their previous results with $m_{\pi} \sim 400 \mathrm{MeV}$ in Ref. [648]. The masses of the low-lying states are generally consistent between these two ensembles within statistical uncertainties. Only the hyperfine splitting $M_{J / \psi}-M_{\eta_{c}}$ has a small but statistically significant increase when the pion mass decreased. For the higher states in the spectrum, the masses are generally larger with the $m_{\pi} \sim 240 \mathrm{MeV}$ ensemble. Especially for the hybrids, there is a small but significant increase in their masses as the pion mass is reduced, which will increase the mass splitting between the hybrids and low-lying conventional charmonium states. However, the statistical uncertainties at higher energies are larger. The unstable nature of these states above threshold may be important [654, 655, 656]. The authors thus concluded that the hybrid mesons appear to show a mild increase in mass as the light quark mass is decreased, but the pattern of states and supermultiplet structure are unchanged.

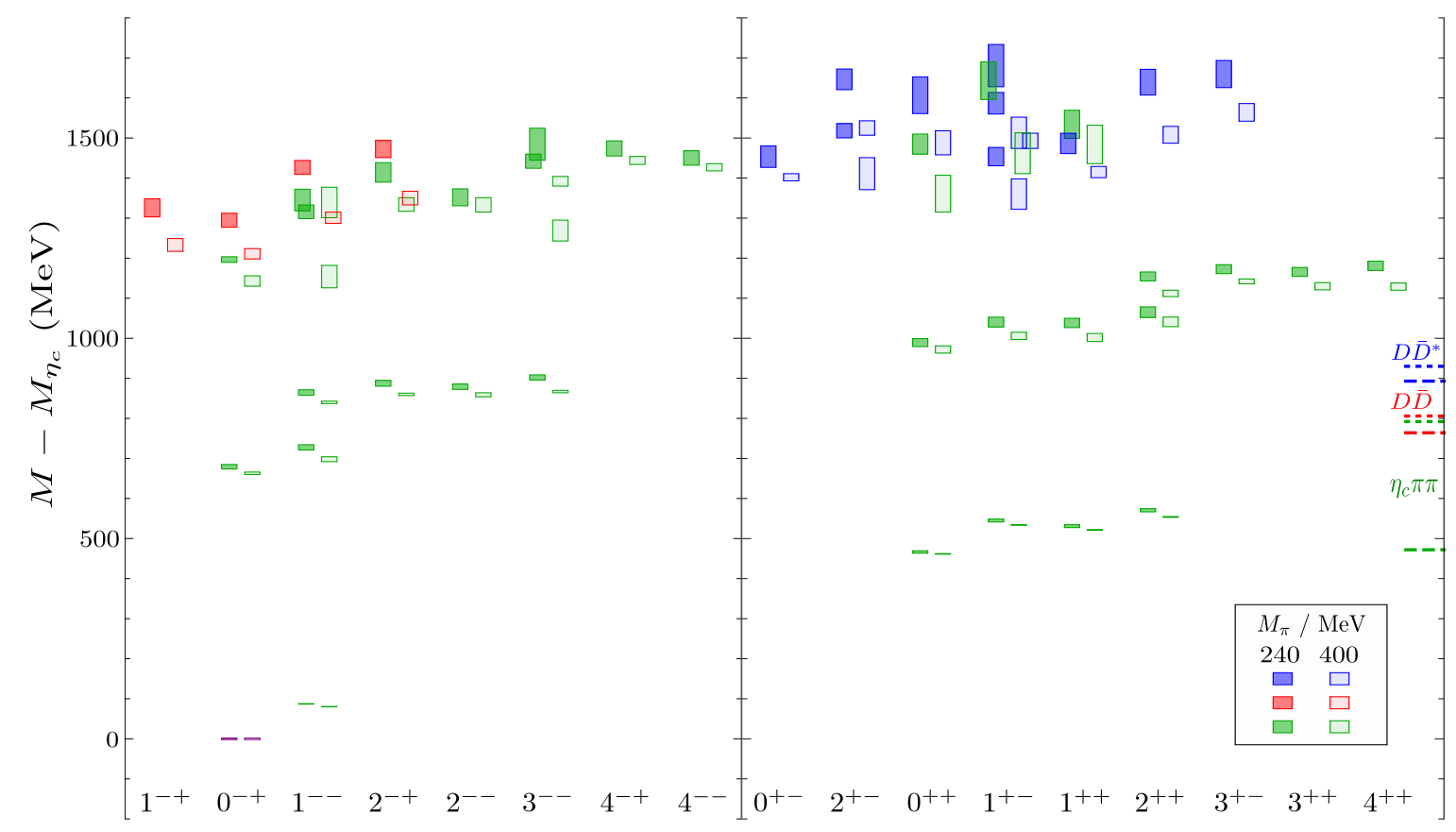

Figure 20: The lattice calculations of charmonium spectra with $m_{\pi} \sim 240 \mathrm{MeV}$ from Ref. [653], comparing to the spectra with $m_{\pi} \sim 400 \mathrm{MeV}$ in Ref. [648]. The green boxes are normal $c \bar{c}$ charmonium states, while red and dark blue boxes are $\bar{c} g c$ charmonium hybrid candidates of the lightest and firstexcited supermultiplet respectively. The dashed lines indicate the corresponding non-interacting hadron thresholds.

In Ref. [657], Chen et al. investigated the existence of the exotic vector charmonia by constructing the interpolating operators $O_{i}^{(H)}(x, t ; r)=\left(\bar{c}^{a} \gamma_{5} c^{b}\right)(x, t) B_{i}^{a b}(x+r, t)$, where $i$ is the spatial index and $B_{i}^{a b}(x)=\frac{1}{2} \epsilon_{i j k} F_{j k}^{a b}$ the chromomagnetic field tensor. Compared to the usual hybrid operator, this is a new type of the hybrid-like operator that the charm quark-antiquark pair component $\bar{c}^{a} \gamma_{5} c^{b}$ and the gluon field $B_{i}^{a b}$ are split into two parts by an explicit spatial displacement $r$. Such a configuration can resemble the center-of-mass motion of the $\bar{c} c$ recoiling against an additional degree of freedom, and thus is expected to suppress the coupling to the conventional charmonia. They accordingly calculated the 
two-point correlation functions $C^{H}(r, t)$ by using these operators. To eliminate the contribution from the conventional charmonium states, they combined linearly the correlation functions at two specific different $r$ as $C(\omega, t)=C^{H}\left(r_{1}, t\right)-\omega C^{H}\left(r_{2}, t\right)$, where $\omega$ is a tunable parameter. For the numerical analyses, they used the tadpole-improved gauge action $[658,659,660]$ to generate gauge configurations on anisotropic lattices. Two lattices $L^{3} \times T=8^{3} \times 96(\beta=2.4)$ and $12^{3} \times 144(\beta=2.8)$ with different lattice spacings were used to check the discretization artifacts. After fitting the effective mass plateaus as shown in Fig. 21, they observed a vector charmonium-like state $X$ with a mass of $4.33(2) \mathrm{GeV}$ with exotic nature. In addition, they calculated the leptonic decay constant of this exotic state to be $f_{X}<40$ $\mathrm{MeV}$, which accordingly led to a very small leptonic decay width $\Gamma\left(X \rightarrow e^{+} e^{-}\right)<40 \mathrm{eV}$. The mass and leptonic decay width of this signal are consistent with the production and decay properties of $Y(4260)$.

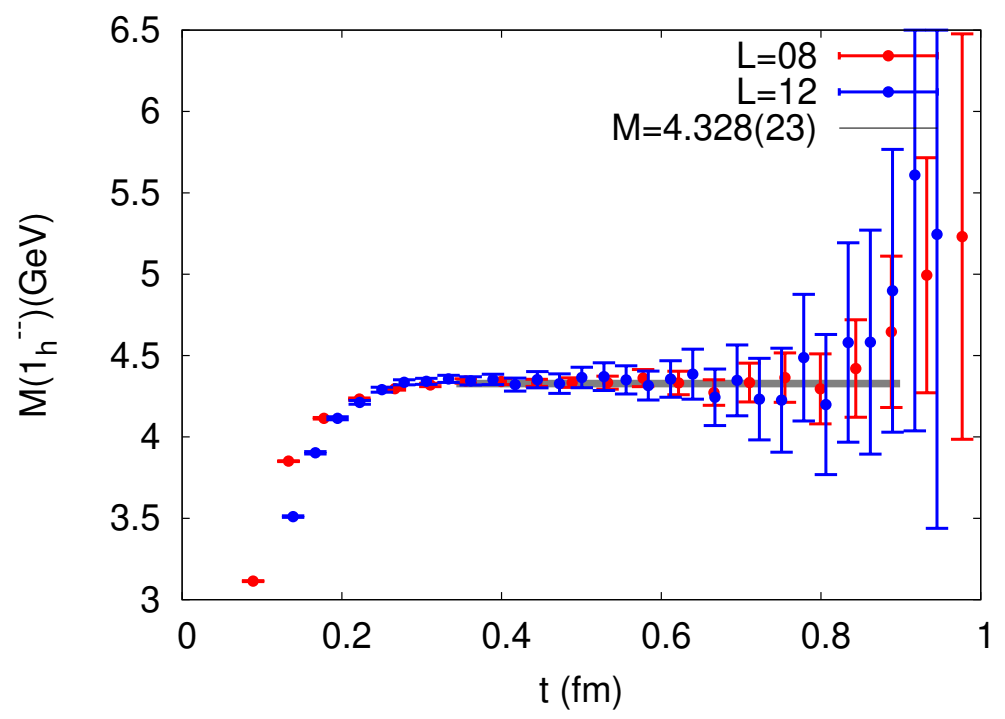

Figure 21: The effective mass plateaus of $C(\omega, t)$ for $\beta=2.4$ (red points) and $\beta=2.8$ (blue points), taken from Ref. [657]

\section{$9.2 X(3872)$}

The TWQCD Collaboration also studied the $X(3872)$ state as a hidden-charm four-quark $q c \bar{q} \bar{c}$ meson with $J^{P C}=1^{++}$in quenched lattice QCD with exact chiral symmetry [661]. They used both molecular operator $\left(\bar{q} \gamma_{i} c\right)\left(\bar{c} \gamma_{5} q\right)-\left(\bar{c} \gamma_{i} q\right)\left(\bar{q} \gamma_{5} c\right)$ and tetraquark operator $\left(q^{T} C \gamma_{i} c\right)\left(\bar{q} C \gamma_{5} \bar{c}^{T}\right)-\left(\bar{q}^{T} C \gamma_{i} \bar{c}\right)\left(q C \gamma_{5} c^{T}\right)$ to compute the time-correlation functions for two lattice volumes $24^{3} \times 48$ and $20^{3} \times 40$. For both the molecular and tetraquark operators, they detected a resonance with mass around $3890 \pm 30 \mathrm{MeV}$ in the limit $m_{q} \rightarrow m_{u}$, which is identified as $X(3872)$. Their results showed that $X(3872)$ has good overlap with the molecular operator as well as the tetraquark operator in the quenched approximation. Comparing with their previous study for $Y(4260)$, they concluded that $X(3872)$ may be more tightly bound than $Y(4260)$, which had better overlap with the molecular operator than any tetraquark ones [643]. Since only one energy level was extracted near the $D \bar{D}^{*}$ threshold, the result could not support or disfavor the existence of $X(3872)$. Letting $m_{q} \rightarrow m_{s}$, they predicted a heavier $1^{++} c s \bar{c} \bar{s}$ exotic meson around $4100 \pm 50 \mathrm{MeV}$. By using the same method and interpolating currents as above, they investigated the mass spectrum of the $(c s \bar{c} \bar{q}) /(c q \bar{c} \bar{s})$ tetraquark states with $J^{P}=1^{+}$. They also detected an axial-vector $(c s \bar{c} \bar{q}) /(c q \bar{c} \bar{s})$ resonance around $4010 \pm 50 \mathrm{MeV}$ [662]. In these investigations of TWQCD Collaboration, the authors neglected the disconnected quark loop diagrams for annihilation channels. However, these diagrams provide the mixing effects between states created by $\bar{c} c$ and $\bar{c} q \bar{q} c$ operators, which have been proven to be important for the lattice studies of light scalar tetraquarks [663, 664], and the string breaking in the static limit [665]. 
As indicated above, the mass spectra of the highly excited charmonium states have been calculated up to around 4.5 GeV in dynamical lattice QCD by the Hadron Spectrum Collaboration in Ref. [648]. The first radial excitation of the $\mathrm{P}$-wave $1^{++}$charmonium state is extracted about $110 \mathrm{MeV}$ above the mass of $X(3872)$, where the mass of $\eta_{c}$ is subtracted from the calculated mass in order to reduce the systematic error from the tuning of the bare charm quark mass. However, the results presented in Ref. [648] are for unphysically-heavy up and down quarks corresponding to $m_{\pi} \approx 400 \mathrm{MeV}$. The dynamical lattice simulation with lighter up and down quarks $m_{\pi} \approx 240 \mathrm{MeV}$ showed that only a very small mass increase for the low-lying and even higher charmonium states [653]. Moreover, several other lattice simulations found one excited $1^{++}$charmonium state near the mass of $X(3872)[666,667,668]$. However, none of these simulations can unambiguously determine whether this state is $X(3872)$ or the scattering $D \bar{D}^{*}$ state.

In Ref. [669], the authors calculated the charmonium spectrum including higher spin and gluonic excitations. In particular, they studied the mixing of charmonia with states created by the hiddencharm molecular operators by using the variational generalized eigenvalue method as well as improved stochastic all-to-all propagator methods. Their simulations were employed with light pseudoscalar mass down to $280 \mathrm{MeV}$ and $n_{F}=2$ sea quarks. They investigated the binding between pairs of $D$ and anti- $D$ mesons in the pseudoscalar, vector and axialvector sectors. Only the axialvector channel was clearly attractive. Moreover, the mixing effects between the isoscalar radial charmonium states and $\bar{c} q \bar{q} c$ molecular states were very small for the pseudoscalar and vector channels. But for the $1^{++}$ channel, they found a significant binding $m_{D^{*} \bar{D}}-\left(m_{D^{*}}+m_{\bar{D}}\right)=88(26) \mathrm{MeV}$. This binding is much bigger than the mass difference $m_{X(3872)}-\left(m_{D^{*}}+m_{\bar{D}}\right)$.

For the molecular interpretation of $X(3872)$, many dynamical studies supported the attractive channel of $D$ and $\bar{D}^{*}$ mesons. One can consult Refs. [1, 72] and references therein for these discussions. Due to the coupled channel effects, the $S$-wave $D \bar{D}^{*}$ scattering state with $J^{P C}=1^{++}$can easily couple to the $\chi_{c 1}^{\prime}(2 P)$ state $[670,671]$ and significantly lower the mass of the pure $\chi_{c 1}^{\prime}(2 P)$ state predicted in the GI model [33].

In lattice QCD, this picture was supported by the dynamical $N_{f}=2$ lattice simulation in Ref. [672], which was based on one ensemble of Clover-Wilson dynamical configurations with $m_{\pi}=266 \mathrm{MeV}$. The authors identified the low-lying $\chi_{c 1}(1 P)$ and $X(3872)$ as well as the nearby discrete scattering levels $D \bar{D}^{*}$ and $J / \psi \omega$ for both $I=0$ and $I=1$ channels. They counted the number of lattice states near the $D \bar{D}^{*}$ threshold in order to establish the existence of the $X(3872)$. In their simulation, they chose the interpolating fields $\mathcal{O}_{i}$ that couple to $\bar{c} c$ as well as the scattering states, i.e., $\mathcal{O}_{1-8}^{\bar{c} c}, \mathcal{O}_{i}^{D D^{*}}(i=1,2,3)$, $\mathcal{O}^{J / \psi \omega}$ (for $I=0$ ), and $\mathcal{O}^{J / \psi \rho}$ (for $I=1$ ). Finally, they found a candidate for the charmoniumlike $X(3872)$ state $11 \pm 7 \mathrm{MeV}$ below the $D \bar{D}^{*}$ with $J^{P C}=1^{++}$and $I=0$, in addition to the nearby $D \bar{D}^{*}$ and $J / \psi \omega$ discrete scattering states, as shown in Fig. 22. They extracted large and negative $D \bar{D}^{*}$ scattering length, $a_{0}^{D D^{*}}=-1.7 \pm 0.4 \mathrm{fm}$, and the effective range, $r_{0}^{D D^{*}}=0.5 \pm 0.1 \mathrm{fm}$. Moreover, they found the $\chi_{c 1}(1 P)$ state but no candidate for the $X(3872)$ in the $I=0$ channel if they used only five scattering interpolators but no $\mathcal{O}_{1-8}^{\bar{c} c}$ interpolating fields. In the $I=1$ channel, they did not find a candidate for $X(3872)$, which is consistent with the assignment of the state in PDG [35].

Later, the isoscalar $J^{P C}=1^{++}$charmonium state $X(3872)$ was also investigated by the Fermilab Lattice and MILC Collaborations on gauge field configurations with $2+1+1$ flavors of highly improved staggered sea quarks (HISQ) with clover (Fermilab interpretation) charm quarks and HISQ light valence quarks [673]. They used a combination of the $c \bar{c}$ and $D \bar{D}^{*}+\bar{D} D^{*}$ interpolating operators. They found an isosinglet candidate of $X(3872)$ with an energy level $13(6) \mathrm{MeV}$ below the $D \bar{D}^{*}$ threshold, which agrees with the result in Ref. [672].

These studies were then extended by Padmanath, Lang, and Prelovsek by utilizing a large basis of the $\bar{c} c$, two-meson and diquark-antidiquark interpolating fields (altogether 22 interpolators) [674]. The Wick contractions were considered including both the connected contraction diagrams and the diagrams for annihilation channels in which the light quarks do not propagate from source to sink. Their simulation 


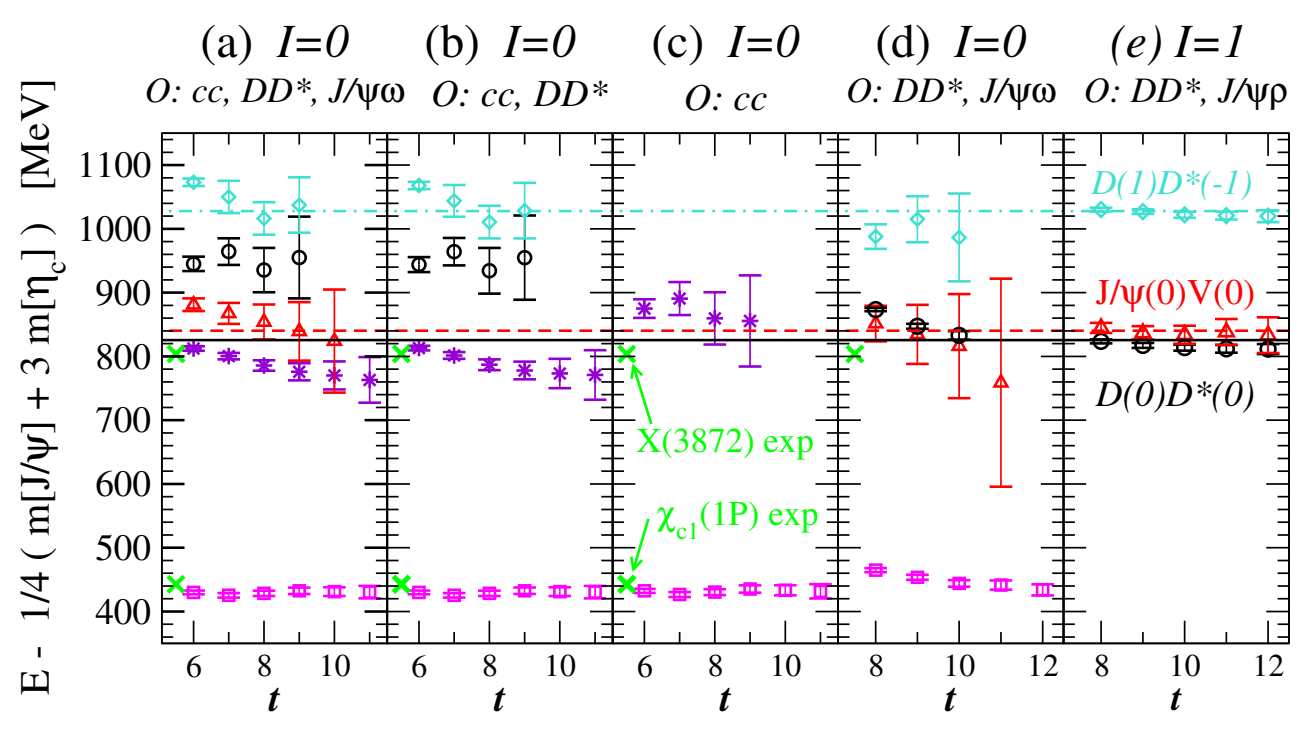

Figure 22: The spectrum $E_{n}-\frac{1}{4}\left(m_{\eta_{c}}+3 m_{J / \psi}\right)$ in the effective mass plateau region for the $J^{P C}=1^{++}$ channel with $I=0$ and $I=1$, from Ref. [672]. Different interpolator basis is chosen for each plot. Dashed lines represent energies $E^{n . i .}$ of the non-interacting scattering states.

was performed with $N_{f}=2$ and $m_{\pi}=266 \mathrm{MeV}$, which aims at the possible charmonium-like $J^{P C}=1^{++}$ signatures for both $I=0$ and $I=1$ channels. They found a lattice candidate for $X(3872)$ with $I=0$ close to the experimental data only if both the $\bar{c} c$ and $D \bar{D}^{*}$ interpolators are included. However, they found no candidate if the diquark-antidiquark and $D \bar{D}^{*}$ interpolators were used in the absence of $\bar{c} c$, which indicates that the $\bar{c} c$ Fock component is crucial for the $X(3872)$ while the four-quark component $O^{4 q}$ alone does not produce the signal. Their simulation also implies a combined dominance of the $\bar{c} c$ and $D \bar{D}^{*}$ operators in determining the position of the energy levels, while the tetraquark $O^{4 q}$ operators do not significantly affect the results. Moreover, their results do not support the neutral or charged $X(3872)$ in the $I=1$ channel. And no signature of the exotic $\bar{c} c \bar{s} s$ state (candidate for the $Y(4140)$ ) with $I=0$ are found below $4.2 \mathrm{GeV}$. We refer readers to Refs. [675, 676, 677, 678] for the analytic investigations considering the quark mass dependence, the volume dependence and the effect from the isospin breaking.

The unphysical pion mass $m_{\pi}=266 \mathrm{MeV}$ on the lattice is still much larger than its physical mass $140 \mathrm{MeV}$. Within the molecular scheme, the long range one-pion-exchange force plays a dominant role in the formation of the loosely bound molecular state $[679,680]$, which decays exponentially as the pion mass increases. The present lattice simulation with the pion mass $m_{\pi}=266 \mathrm{MeV}$ was obtained from a rather small lattice volume, which has already indicated the important role of both the $\bar{c} c$ and $D \bar{D}^{*}$ components in the formation of the $X(3872)$ signal on the lattice. As the pion mass approaches the physical value $140 \mathrm{MeV}$, one shall expect a larger $D \bar{D}^{*}$ component within the physical $X(3872)$ state.

\subsection{The charged $Z_{c}$ states}

The observations of the charged charmonium-like states $Z_{c}(3900)$ and $Z_{c}(4020)$ trigged lots of investigations of the molecular interpretations for their inner structures [680, 681, 682, 683]. In Ref. [684], Prelovsek and Leskovec searched for the $Z_{c}(3900)$ in the $J^{P C}=1^{+-}$and $I=1$ channel in their lattice simulation with degenerate dynamical $u / d$ quarks and $m_{\pi} \approx 266 \mathrm{MeV}$. They used six meson-meson type of interpolators $O_{i}^{D D^{*}}$ and $O_{i}^{J / \psi \pi}(i=1,2,3)$. They calculated the time-dependence of the $6 \times 6$ correlation matrix by considering the connected diagrams of Wick contraction. They omitted the diagrams involving charm annihilation, which effect was proven to be suppressed due to the OZI rule for 
the conventional charmonium [685]. They observed four energy levels which almost exactly coincide with the energies of four non-interacting $D \bar{D}^{*}$ and $J / \psi \pi$ scattering states. They did not find any additional energy level relating to the $Z_{c}(3900)$ state. They suggested to perform simulations including meson-meson interpolators as well as some different type of interpolators, such as diquark-antidiquark interpolators with the same quantum numbers.

Such a simulation was performed in Ref. [686] by using 18 meson-meson $O^{M_{1} M_{2}}$ interpolating operators and 4 diquark-antidiquark $O^{4 q}$ interpolators with structure $[\bar{c} \bar{d}]_{3_{c}}[c u]_{\overline{3}_{c}}$ in the $I^{G} J^{P C}=1^{+} 1^{+-}$ channel. The two-meson operators $O^{M_{1} M_{2}}$ included interpolators such as $J / \psi \pi, \eta_{c} \rho, D \bar{D}^{*}$ and $D^{*} \bar{D}^{*}$. They aimed to extract and identify all 13 two-meson energy levels from the corresponding $22 \times 22$ correlation functions and examine the possible additional states related to the exotic $Z_{c}^{+}$hadron. As a result, they found all expected lowest thirteen levels, appearing near the corresponding non-interacting energies of the two-particle states. A two-particle level will disappear from the spectrum or become very noisy when the corresponding $O^{M_{1} M_{2}}$ operator is absent from the correlator matrix. However, the energy spectrum was unaffected after the $O_{1-4}^{4 q}$ operators were omitted. Besides these two-particle levels, they did not find any additional state below $4.2 \mathrm{GeV}$ relating to an exotic $Z_{c}^{+}$candidate. They listed some possible reasons for the absence of the $Z_{c}^{+}$candidate: a) the experimental resonance $Z_{c}^{+}(3900)$ might not be of dynamical origin since it has not been seen in the $B$ meson decay process; b) it might be a couple-channel threshold effect $[687,688]$; c) their simulation was performed at a large unphysical $m_{\pi} \approx 266 \mathrm{MeV} ; \mathrm{d}$ ) their interpolator basis may not be complete enough to render a $Z_{c}$ state in addition to thirteen two-meson states; e) the S-D mixing effect may be important for creating the experimental $Z_{c}$ states. In Ref. [673], the authors used the Highly Improved Staggered Quark action to search for the $Z_{c}(3900)$ with a combination of the $J / \psi \pi$ and $D \bar{D}^{*}+\bar{D} D^{*}$ channels. Again, they found no evidence for the existence of $Z_{c}(3900)$. A similar analysis was performed in Ref. [689] by considering a basis of five $I=0 D^{(*)} \bar{D}^{*}$ interpolating operators and three $I=1 D \bar{D}^{*}$ operators with the same $J^{P}=1^{+}$. Their results didn't show any unknown energy level.

These negative results of searching for the exotic charged $Z_{c}$ in lattice QCD simulations were also supported by the investigations of the resonance-like $Z_{c}$ structures in the low-energy scattering of the $\left(D^{(*)} \bar{D}^{*}\right)^{ \pm}$systems by the CLQCD Collaboration [690, 691]. In Ref. [690], Chen et al. presented an exploratory lattice study of the low-energy scattering of the $\left(D \bar{D}^{*}\right)^{ \pm}$two-meson system by using singlechannel Lüscher's finite-size technique. The calculation was based on $N_{f}=2$ twisted mass fermion configuration of size $32^{3} \times 64$ with a lattice spacing of about $0.067 \mathrm{fm}$ with three pion mass values $m_{\pi}=300 \mathrm{MeV}, 420 \mathrm{MeV}$ and $485 \mathrm{MeV}$. They constructed the $S$-wave two-meson operators with $I^{G} J^{P C}=1^{+} 1^{+-}$to calculate the correlation functions. Since the energy being considered is very close to the $\left(D \bar{D}^{*}\right)^{ \pm}$threshold, they computed the scattering length $a_{0}$ and effective range $r_{0}$ to search for the evidence of the existence of $Z_{c}(3900)$. They found negative values of the scattering length $a_{0}$ for all three pion masses, which indicated a weak repulsive interaction between the two mesons $D$ and $\bar{D}^{*}$. They also checked the possibility of the bound state for the negative energy shifts. None of them was consistent with the signal of a bound state. Based on their results, they concluded that their lattice QCD simulation did not support a bound state in the $I^{G} J^{P C}=1^{+} 1^{+-}$channel corresponding to $Z_{c}(3900)$, at least for the pion mass values being studied. Later in Ref. [691], they performed a similar investigation for the low-energy scattering of the $D^{*} \bar{D}^{*}$ two-meson system in the same channel with $I^{G} J^{P C}=1^{+} 1^{+-}$. Their results gave negative values of the scattering lengths for all three pion masses, which again indicated a weak repulsive interaction between the two vector charmed mesons, and did not support a bound state of the two mesons in the $J^{P}=1^{+}$channel corresponding to the $Z_{c}(4020) / Z_{c}(4025)$ state.

The lattice QCD studies of the spectrum and the low-energy scattering parameters with the standard Lüscher's method gave no candidates for the $Z_{c}(3900)$ and $Z_{c}(4025)$ in all $J / \psi \pi, \eta_{c} \rho, D \bar{D}^{*}$ and $D^{*} \bar{D}^{*}$ two-meson channels. These results indicated that the $Z_{c}(3900)$ and $Z_{c}(4025)$ may not be the conventional resonance states. In Refs. [692, 693], the HAL QCD Collaboration studied the 
$\pi J / \psi-\rho \eta_{c}-\bar{D} D^{*}$ coupled-channel interactions from $(2+1)$-flavor full QCD simulations at three pion masses $m_{\pi}=411 \mathrm{MeV}, 570 \mathrm{MeV}, 701 \mathrm{MeV}$ in order to explore the structure of $Z_{c}(3900)$. To consider the interactions among the $\pi J / \psi, \rho \eta_{c}$ and $\bar{D} D^{*}$ channels, the authors calculated the $S$ matrix from the equal-time Nambu-Bethe-Salpeter (NBS) wave functions by using the coupled-channel HAL QCD method [694, 695, 696]. They extracted the $s$-wave coupled-channel potential $V^{\alpha \beta}$ and found that all diagonal potentials $V^{\bar{D} D^{*}, \bar{D} D^{*}}, V^{\rho \eta_{c}, \rho \eta_{c}}$ and $V^{\pi J / \psi, \pi J / \psi}$ were very weak, which indicated that the $Z_{c}(3900)$ was neither a simple $\pi J / \psi$ hadrocharmonium nor $D D^{*}$ molecule state. Instead, they found the $\rho \eta_{c}-\bar{D} D^{*}$ coupling and the $\pi J / \psi-\bar{D} D^{*}$ coupling from the off-diagonal potentials were both strong, which implied that the structure of $Z_{c}(3900)$ can be explained as a threshold cusp. They further calculated the invariant mass spectra and pole positions associated with the coupled-channel two-body $T$ matrix on the basis of $V^{\alpha \beta}$. Their results supported the $Z_{c}(3900)$ resonance as a threshold cusp induced by the strong $\pi J / \psi-\bar{D} D^{*}$ coupling. They further made a semiphenomenological analysis of the three-body decays $Y(4260) \rightarrow \pi \pi J / \psi, \pi \bar{D} D^{*}$ processes. As shown in Fig. 23, they found that the coupled-channel potential $V^{\alpha \beta}$ can well reproduce the experimental peak structures in the $Y(4260) \rightarrow \pi \pi J / \psi$ and $Y(4260) \rightarrow \pi \bar{D} D^{*}$ decays. When the off-diagonal components of $V^{\alpha \beta}$ were turned off, the peak structures at $3.9 \mathrm{GeV}$ disappeared in both cases. Accordingly, the authors concluded that the $Z_{c}(3900)$ was not a conventional resonance but a threshold cusp [692, 693].

As indicated in Refs. [684, 686], including a large basis of four-quark interpolating operators in the calculations could lead to more reliable determination of finite-volume mass spectra and scattering amplitudes. In the very recent lattice QCD study [697], the Hadron Spectrum Collaboration constructed 29 hidden-charm interpolating currents and 48 doubly-charmed interpolating currents with different colorflavor-spatial-spin structures. Using these diverse bases of meson-meson operators as well as compact tetraquark operators, they computed the two-point correlation functions in the isospin-1 hidden-charm and doubly-charmed sectors using the distillation framework [698]. Their calculations were performed on an anisotropic $16^{3} \times 128$ lattice volume using 478 configurations and a Clover fermion action with $N_{f}=2+1$ flavors of dynamical quarks. The mass of the two degenerate light quarks corresponds to $m_{\pi}=391 \mathrm{MeV}$ while the strange quark was tuned so that its mass approximated the physical value [699]. The multiple energy levels associated with the meson-meson levels which could be degenerate in the non-interacting limit were extracted reliably. In Fig. 24, the mass spectra in the isospin-1 hidden-charm sector were shown for the lattice irreps $\Lambda^{P G}=T_{1}^{++}, A_{1}^{+-}, T_{1}^{+-}$corresponding to the $I=1$ and $J^{P C}=1^{+-}, 0^{++}, 1^{++}$for the charge neutral channels, respectively. One found that every energy level had a dominant overlap onto one meson-meson operator, while the addition of tetraquark operators to a basis of meson-meson operators did not significantly affect the finite-volume spectrum. For all these channels, the number of energy levels in the computed spectra was equal to the number of non-interacting two-meson levels expected in the considered energy region and they all lie close to the non-interacting levels. The authors concluded that there were no strong indications for any bound state or narrow resonance in these channels [697]. Especially for the isovector $T_{1}^{++}$irreps with $J^{P C}=1^{+-}$, they didn't find any candidate for the $Z_{c}(3900)$ and $Z_{c}(4025)$ states. Since the $X(3872)$ has been admitted as an isosinglet [35], this study gave no hint for its existence. However, if the $X(3872)$ exists as a tetraquark $[672,673,674]$, its isospin-1 partner would appear in this channel with $I^{G} J^{P C}=1^{+} 1^{+-}$. However, no clear signal for such an isovector hidden-charm tetraquark state was found in Ref. [697].

Besides the studies of $Z_{c}(3900)$ and $Z_{c}(4025)$ in low-energy scattering of the $\left(D^{(*)} \bar{D}^{*}\right)^{ \pm}$systems, the CLQCD Collaboration also investigated the low-energy near threshold scattering of the $\left(\bar{D}_{1} D^{*}\right)^{ \pm}$system in both the $S$-wave $\left(A_{1}\right.$ with $\left.I^{G} J^{P C}=1^{+} 0^{--}\right)$and $P$-wave $\left(T_{1}\right.$ with $\left.I^{G} J^{P C}=1^{+} 1^{+-}\right)$channels $[700$, 701]. In Ref. [701], the authors performed their calculations by using lattice QCD with $N_{f}=2$ twisted mass fermion configurations of size $32^{3}$ with lattice spacing $a \approx 0.067 \mathrm{fm}$ at three pion masses $m_{\pi}=307.0$ $\mathrm{MeV}, 423.6 \mathrm{MeV}, 488.4 \mathrm{MeV}$. For the $\bar{D}_{1} D^{*}$ scattering in the $S$-wave $A_{1}$ channel, they obtained the values of the lowest $q^{2}$ to be in the range $[-0.7,-0.5]$, which increased by an order of magnitude compared with their previous quenched lattice QCD result in Ref. [700]. This resulted in stronger 

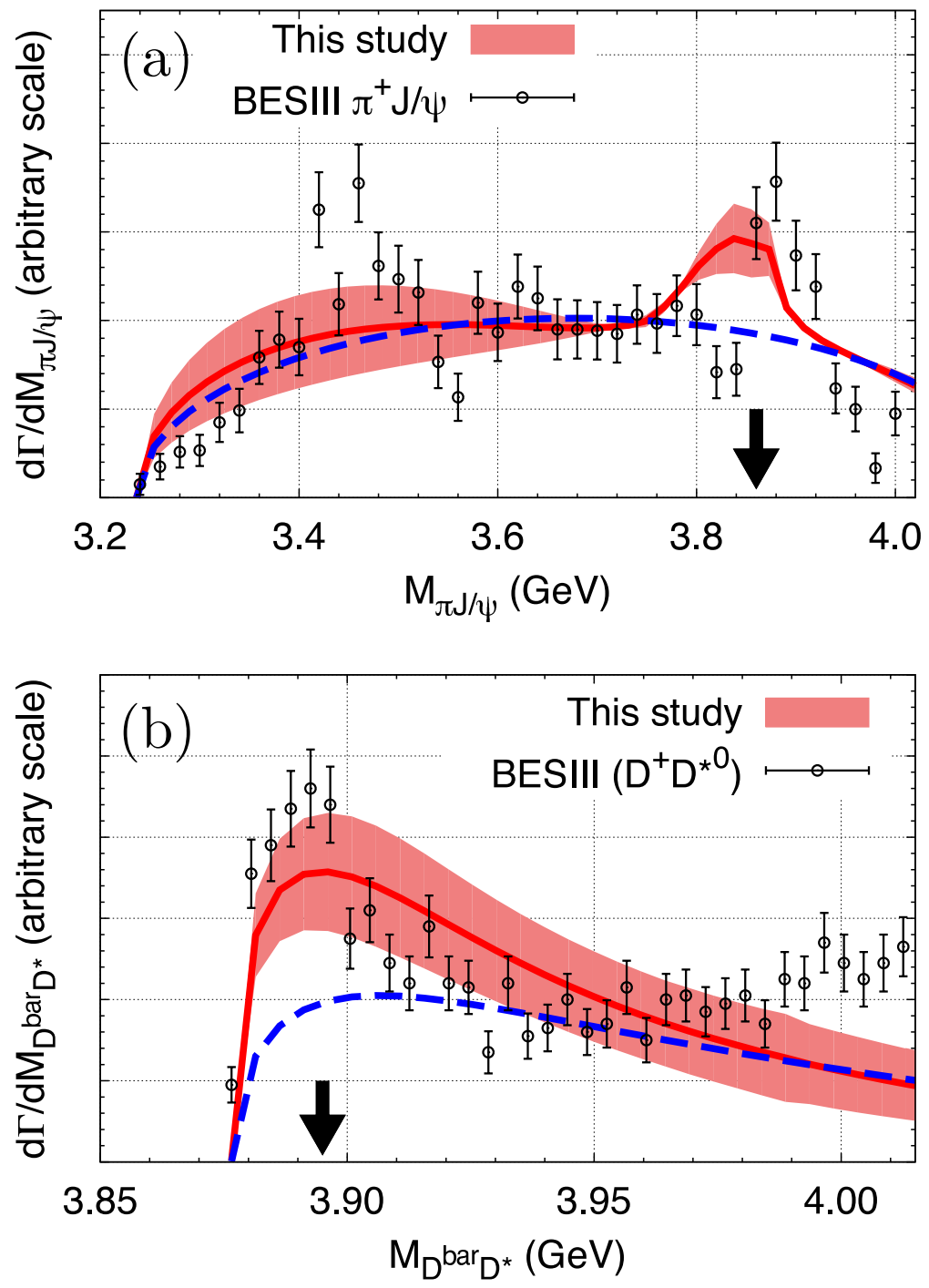

Figure 23: The invariant mass spectrum of (a) $Y(4260) \rightarrow \pi \pi J / \psi$ and (b) $Y(4260) \rightarrow \pi \bar{D} D^{*}$ established with the coupled-channel potential $V^{\alpha \beta}$ from the lattice QCD simulation at $m_{\pi}=411 \mathrm{MeV}$, taken from Ref. [692]. The vertical black arrows show the reproduced peak positions from the lattice QCD calculations. The blue dashed lines show the invariant mass spectra without the off-diagonal components of $V^{\alpha \beta}$.

attractive interaction between the two charmed mesons. The phase shift was also checked to satisfy $\cot \delta\left(q^{2}\right) \approx-1$, which confirmed the existence of a shallow bound state in this channel. In the $P$-wave $T_{1}$ channel, similar conclusions were reached by inspecting the lowest values of $q^{2}$ and the quantity $\cot \delta\left(q^{2}\right)$. Based on these results, the author concluded that the interactions between a $\left(\bar{D}_{1} D^{*}\right)^{ \pm}$system are attractive and a possible bound state below the threshold may exist for both pseudoscalar and axial-vector channels. Especially, the resonance candidate in the $I^{G} J^{P C}=1^{+} 1^{+-}$channel may provide some hints on the nature of the $Z^{+}(4430)$ state [701]. 


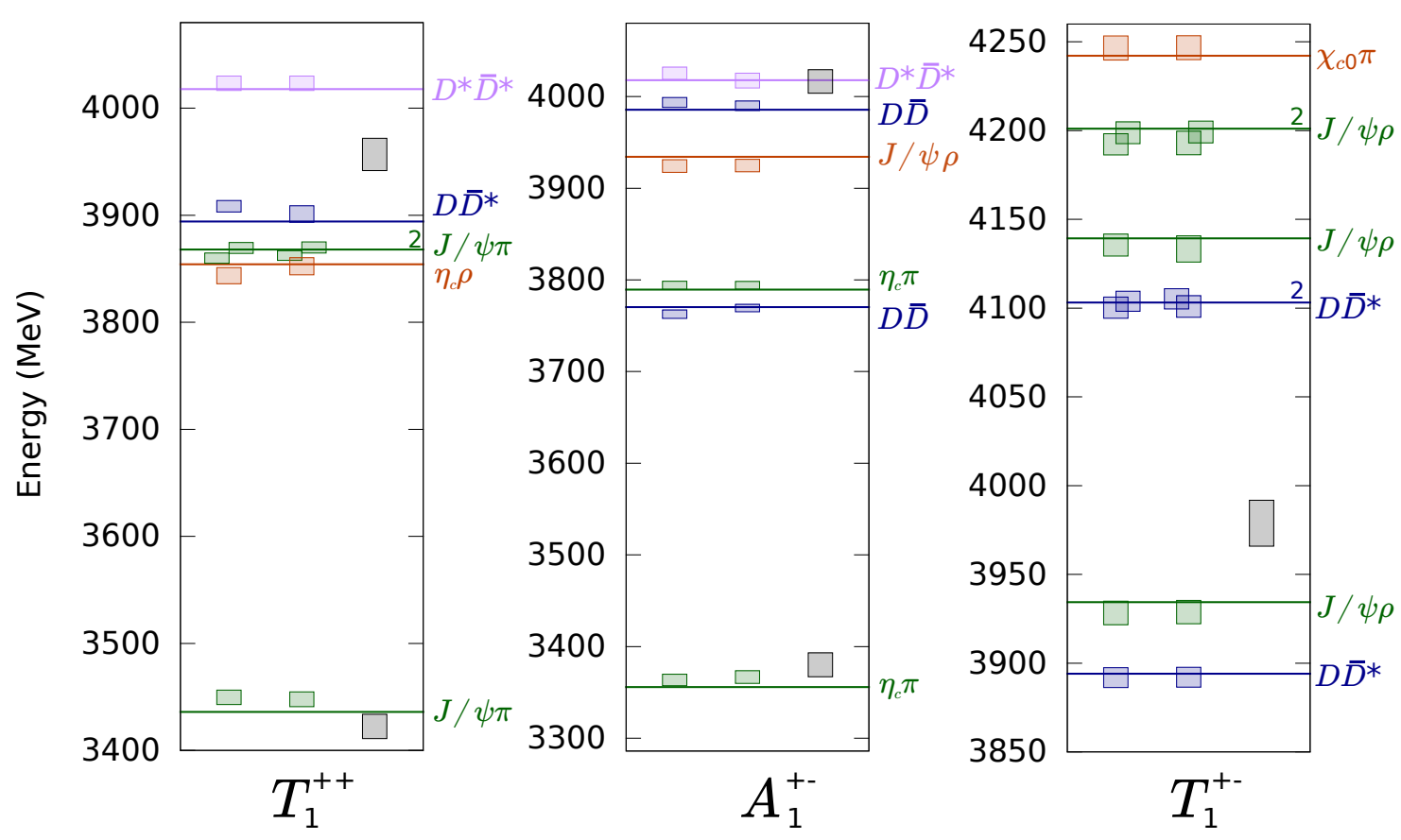

Figure 24: Figure was taken from Ref. [697]. The spectrum plot in the hidden-charm isospin-1 $\Lambda^{P G}=T_{1}^{++}, A_{1}^{+-}, T_{1}^{+-}$lattice irreps calculated using the full basis of meson-meson and tetraquark operators (left column), only meson-meson operators (middle column) and only tetraquark operators (right column). Horizontal lines denote the non-interacting meson-meson energy levels, and boxes give the lattice QCD computed energies.

\subsection{The doubly-charmed/bottom tetraquark states}

The idea of searching for stable tetraquark states from the first principle lattice QCD has a long history and is still attractive now. In 1990's, there were some efforts to calculate the interactions and potential between two heavy-light mesons in lattice QCD [702, 703, 155, 156, 704, 705], inspired by many phenomenological studies of the stability for these systems $[173,142,141,144,706,145,147,707$, $150,151,152]$.

In Ref. [703], the authors extracted an effective potential between the heavy-light mesons (HLHL systems) from the quark correlation functions in the framework of quenched lattice QCD with KogutSusskind fermions. The correlation functions were calculated by including the two kinds of diagrams corresponding to the pure gluon-exchange part and the quark-exchange part of the mesonic interactions. The gauge field configurations were generated on a periodic $8^{3} \times 16$ lattice with inverse gauge coupling $\beta=5.6$ corresponding to a lattice spacing $a \approx 0.19 \mathrm{fm}$. Finally, they were able to extract the $M M$ potential from the Euclidean time behavior of these correlators. The result showed that the resulting potential was attractive at short heavy-quark distances and the interaction was stronger for smaller light quark masses. They also found that the quark-exchange diagram played a significant role only for distance $r$ much less than $2 a$. This result was supported by the investigation in Ref. [155], where an adiabatic approximation was used to derive the binding energy potential between two heavy-light mesons in quenched SU(2)-color lattice QCD. The derived binding potential was attractive at short and medium range. The UKQCD Collaboration studied the $B B$ system at fixed heavy quark separation $R$ in quenched [156] and unquenched [704] SU(3) lattice QCD. They found evidence for deep binding at small $R$ in the $I_{q}, S_{q}=(0,0)$ and $(1,1)$ (where $I_{q}$ and $S_{q}$ are total isospin and spin for the two light quarks, respectively) cases, for both quenched and unquenched results. The binding energy was about $200-400 \mathrm{MeV}$ at $R=0$ and very short-ranged. It was essentially contributed by the gluon- 
exchange diagram and insensitive to the light quark mass. The $(0,1)$ channel at $R=0$ was attractive for unquenched and repulsive for quenched, which was the only difference between the quenched and unquenched results. The quark-exchange diagram contributed at larger separations $R \approx 0.5 \mathrm{fm}$, where the evidence for weak binding was found. The authors concluded that the exotic $b b \bar{q} \bar{q}$-mesons exist as states stable under strong interactions.

However, the extracted potentials remain largely unexplored in the above lattice calculations due to the large statistical uncertainties. In Ref. [708], the NPLQCD Collaboration studied the potentials between two $B$ mesons in the heavy-quark limit by choosing a relatively small lattice volume in order to explore the intermediate and short-distance component of the potential. Their calculations were performed on $16^{3} \times 32$ quenched lattices with a spatial length of $\sim 1.6 \mathrm{fm}$ at a pion mass $m_{\pi} \approx 400$ $\mathrm{MeV}$. They found nonzero central potentials in all four $\left(I, s_{l}\right)=(0,0),(0,1),(1,0),(1,1)$ spin-isospin channels, where $s_{l}$ is the total spin of the light quarks. At short distance, they found clear evidence of repulsion between the $B$ mesons in the $I \neq s_{l}$ channels and attraction in the $I=s_{l}$ channels. Later, the possible tetraquark bound states in HLHL system were suggested in Refs. [709, 710], based on their lattice QCD calculations of the interaction potentials for various channels in the heavy-quark limit.

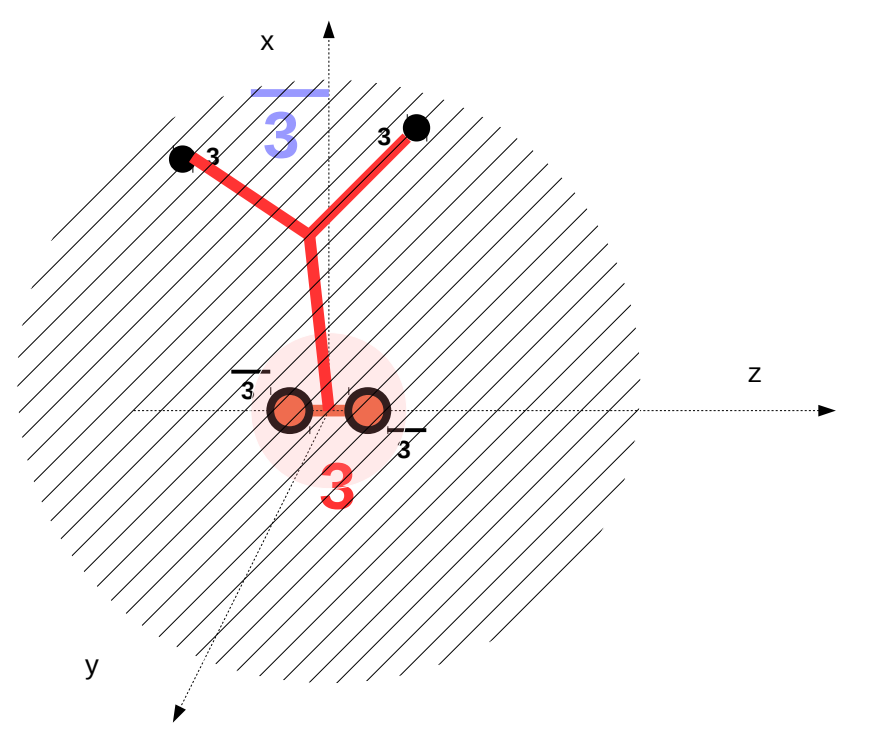

Figure 25: The $\bar{b} \bar{b}$ pair forms a color triplet antidiquark at small separation $r$. The screening of the light quarks has essentially little effect on the $\bar{b} \bar{b}$ interaction, due to the much farther separated light quarks $q q$. Figure was taken from Ref. [357].

Besides, the $B B$ potential was also computed by using the Wilson twisted mass lattice QCD with two flavors of degenerate dynamical quarks $\left(m_{\pi} \approx 340 \mathrm{MeV}\right)[711,712]$. The authors calculated 36 independent trial state potentials using $24^{3} \times 48$ gauge field configurations. They established a simple rule to judge whether a $B B$ potential is attractive or repulsive: a $B B$ potential is attractive if the trial state is symmetric under the meson exchange, while the potential is repulsive if the trial state is antisymmetric under the meson exchange, where the meson exchange means the combined exchange of flavor, spin and parity. Using the dynamical results obtained in Refs. [711, 712], Bicudo and Wagner found strong indication for the existence of a $\bar{b} \bar{b} q q$ tetraquark bound state [355]. Assuming that the pair of antibottom quarks was immersed in a cloud of two light quarks whose screening has little effect on the $\bar{b} \bar{b}$ interaction, the authors suggested the following ansatz to model the heavy antiquark-antiquark 
potential:

$$
V(r)=-\frac{\alpha}{r} e^{-(r / d)^{p}}
$$

where $r$ is the separation of two antibottom quarks, $d$ characterizes the size of the $B$ meson and $p$ is an exponent. They focused on a scalar isosinglet and a vector isotriplet tetraquark channels, which were found to have attractive interactions between two static-light mesons in the dynamical lattice simulations [711, 712]. Fitting the ansatz Eq. (113) to the lattice results for the heavy antiquarkantiquark interactions, the authors were able to determine all three parameters $\alpha, d$ and $p$ for the scalar isosinglet while only two parameters $\alpha$ and $d$ for the vector isotriplet. They derived an analytical rule for the existence or non-existence of a bound tetraquark state for the radial equation in three dimensions. Applying this rule to angular momentum $l=0$, they found the condition for having at least one bound state

$$
\mu \alpha d \geq \frac{9 \pi^{2}}{128 \times 2^{1 / p} \Gamma^{2}(1+1 / 2 p)},
$$

where $\mu$ is the reduced antibottom quark mass. According to this condition, the authors only found a strong indication for the existence of a bound state in the isoscalar channel. To investigate the existence of this bound state rigorously, they numerically solved the Schrödinger equation to calculate the binding for heavy $\bar{b} \bar{b} q q$ tetraquarks. They confirmed the existence of this $\bar{b} \bar{b} q q$ tetraquark state with a confidence level of around $1.8 \sigma \ldots 3.0 \sigma$ and binding energy of approximately $30 \mathrm{MeV} \ldots 57 \mathrm{MeV}$. For the vector isotriplet channel, however, both the analytical rule and binding energy obtained from solving Schrödinger equation indicated that the existence of a bound state in this channel is rather questionable. Nevertheless, possible sources of systematic errors should be considered, including the choice of the heavy quark mass and the lattice spacing, the lattice QCD finite volume effects, the unphysically heavy light quark masses.

In Ref. [357], they extended their computations for the light quark combinations $q q \in\{(u d-$ $d u) / \sqrt{2}, u u,(u d+d u) / \sqrt{2}, d d\}[355]$ to similar systems with heavier strange and charm quarks, i.e. $q q=s s$ and $q q=c c$, by investigating and quantifying systematic uncertainties in detail. They confirmed the existence of the isoscalar $u d \bar{b} \bar{b}$ tetraquark state with quantum numbers $I\left(J^{P}\right)=0\left(1^{+}\right)$and no bound tetraquark state in the isotriplet channel with $I\left(J^{P}\right)=1\left(0^{+}\right), 1\left(1^{+}\right), 1\left(2^{+}\right)$. They found no $s s \bar{b} \bar{b}$ and $c c \bar{b} \bar{b}$ tetraquarks exist in any channel. Later they considered the light-quark mass dependence and computed the $B B$ potential as a function of their spatial separation $r$, by using the twisted mass lattice QCD (tmQCD) with a lattice spacing around $0.079 \mathrm{fm}$ and three infinite-volume pion mass $m_{\pi} \approx 340 \mathrm{MeV}$, $480 \mathrm{MeV}, 650 \mathrm{MeV}$ [356]. This allowed for an extrapolation of the potentials to the physical pion mass, showing the tendency that the binding in the scalar isosinglet channel became stronger towards the physical pion mass, while the pion mass dependence was relatively mild close to the physical point. Accordingly, the authors concluded that the two $B$ mesons could form a tetraquark state in the scalar isosinglet channel with the binding energy $E_{B}=-90_{-36}^{+43} \mathrm{MeV}$. For the vector isotriplet channel, there was no binding and the results were essentially independent on the pion mass.

Including the effects of the heavy $\bar{b}$ quark spins, Bicudo et. al. reanalyzed the $u d \bar{b} \bar{b}$ tetraquark binding energy by solving a coupled-channel Schrödinger equation with the Born-Oppenheimer approximation [713]. They found that the spin of heavy $\bar{b}$ quarks decreased the binding energy. However, the attraction was sufficiently strong that the previously predicted $u d \bar{b} \bar{b}$ bound tetraquark state persisted in $I\left(J^{P}\right)=0\left(1^{+}\right)$channel. Later in Ref. [358], a new tetraquark $u d \bar{b} \bar{b}$ resonance with quantum numbers $I\left(J^{P}\right)=0\left(1^{-}\right)$was predicted.

However, the above lattice simulations for the $u d \bar{b} \bar{b}$ tetraquarks were all performed with $m_{\pi}>$ $340 \mathrm{MeV}$ in the static heavy quark limit. Recently, Francis et. al. investigated the possibility of the $u d \bar{b} \bar{b}$ and $l s \bar{b} \bar{b}(l=u, d)$ tetraquark bound states using $n_{f}=2+1$ lattice QCD ensembles with sufficiently low pion masses $m_{\pi} \approx 164,299$ and $415 \mathrm{MeV}$ and the near-physical $m_{K}$ [714]. The authors considered one diquark-antidiquark interpolating operator and a meson-meson operator to calculate 
the correlation functions. They used the NRQCD lattice action $[715,716,717]$ to calculate the bottom quark propagators, which avoided the static approximation for the heavy $\bar{b}$ quarks. Finally, they found unambiguous signals for the $J^{P}=1^{+}$tetraquark states in the $u d \bar{b} \bar{b}$ and $l s \bar{b} \bar{b}$ channels, which lied 189(10) and $98(7) \mathrm{MeV}$ below the corresponding free two-meson thresholds. These $u d \bar{b} \bar{b}$ and $l s \bar{b} \bar{b}$ tetraquarks can only decay via the weak interaction, implying that these states are rather stable. Later in Ref. [718], they extended their investigation to study the $q q^{\prime} \bar{b} \bar{c}$ tetraquark states with the same method. They found the evidence of a stable $I\left(J^{P}\right)=0\left(1^{+}\right) u d \bar{b} \bar{c}$ tetraquark, which lies about $15-61 \mathrm{MeV}$ below the corresponding $\left(\bar{D} B^{*}\right)$ threshold.

There were also some studies on the doubly-charmed tetraquark states in lattice QCD. In Ref. [719], Ikeda et. al. investigated the S-wave meson-meson interactions in the $D-D, \bar{K}-D, D-D^{*}$ and $\bar{K}-D^{*}$ systems with both $I=0$ and $I=1$, by using $(2+1)$-flavor full QCD gauge configurations generated at $m_{\pi}=410 \sim 700 \mathrm{MeV}$. They employed the relativistic heavy-quark action to treat the charm quark dynamics on the lattice. Using the HAL QCD method, the authors extracted the S-wave potentials in lattice QCD simulations and then calculated the meson-meson scattering phase shifts and scattering lengths. Their results showed that the interactions were repulsive and insensitive to the pion mass for the $I=1$ channels while they were attractive and growing with $m_{\pi}$ for the $I=0$ channels. However, the S-wave scattering phase shifts in these attractive channels indicated that no bound states or resonances were formed at the pion mass $m_{\pi}=410 \sim 700 \mathrm{MeV}$, particularly in the $I=0 D-D^{*}$ channel corresponding to the doubly-charmed tetraquarks $T_{c c}$ with quantum numbers $I\left(J^{P}\right)=0\left(1^{+}\right)$.

The mass spectra for the doubly-charmed tetraquark states were also studied by the Hadron Spectrum Collaboration, using 48 interpolating currents [697]. Using these diverse bases of the meson-meson operators as well as compact tetraquark operators, they calculated the mass spectra for the flavor content $c c \bar{l}$ with isospin-0 in the lattice irreps $\Lambda^{P}=T_{1}^{+}, E^{+}, T_{2}^{+}$corresponding to $J^{P}=1^{+}, 2^{+}, 2^{+}$respectively as shown in Fig. 26, and mass spectra for the flavor $c c \bar{l} \bar{s}$ with isospin- $\frac{1}{2}$ in the irreps $\Lambda^{P}=A_{1}^{+}, T_{1}^{+}$ corresponding to $J^{P}=0^{+}, 1^{+}$respectively as shown in Fig. 27. Similar to the results for the isospin-1 hidden-charm sector, the number of energy levels in the computed spectra for the doubly-charmed sector was equal to the number of non-interacting two-meson levels and the majority of energies were at most slightly shifted from the non-interacting levels. There were no strong indications that there was any bound state or narrow resonance for the $T_{c c}$ tetraquarks in these channels [697].

A comprehensive lattice QCD calculation of the tetraquark states with quark contents $q_{1} q_{2} \bar{Q} \bar{Q}, q_{1}, q_{2} \subset$ $u, d, s, c$ and $Q \equiv b, c$ in both spin zero $(J=0)$ and spin one $(J=1)$ sectors was presented on three dynamical $N_{f}=2+1+1$ highly improved staggered quark ensembles at lattice spacings of about $0.12,0.09$, and $0.06 \mathrm{fm}$ [720]. The authors employed the overlap fermion action for the light, strange, and charm quarks [721, 722] while a NRQCD formulation for the bottom quark [716]. They performed the standard method for GEVP to obtain the effective masses of the ground state energy levels. The results of the spin one tetraquark states showed the presence of the ground state energy levels which were below their respective thresholds for all $u d \bar{b} \bar{b}, u s \bar{b} \bar{b}, u c \bar{b} \bar{b}, s c \bar{b} \bar{b}$ and $u d \bar{c} \bar{c}, u s \bar{c} \bar{c}$ light flavor combinations. The mass spectra for all spin zero tetraquark ground state were found to lie above their respective thresholds, strongly disfavor the existence of any heavy bound tetraquark state with spin zero.

\subsection{Other tetraquark configurations}

After the $X(5568)$ was observed by the D0 Collaboration, Lang et. al. investigated the $S$-wave $B_{s} \pi^{+}$scattering using lattice QCD to search for its existence with the flavor $\bar{b} s \bar{d} u$ and spin-parity $J^{P}=0^{+}$[723]. They employed gauge configurations with $N_{f}=2+1$ dynamical quarks and lattice spacing $a=0.0907(13) \mathrm{fm}$ at rather low pion mass $m_{\pi}=162.6(2.2)(2.3) \mathrm{MeV}$. For the strange quark, they used a partially quenched setup with the valence mass close to the physical point leading to $m_{K}=504(1)(7) \mathrm{MeV}$ [724]. The bottom quark was treated as a valence quark using the Fermilab method $[725,726]$. They took into account the $B^{+} \bar{K}^{0}$ channel for completeness. They didn't find a 


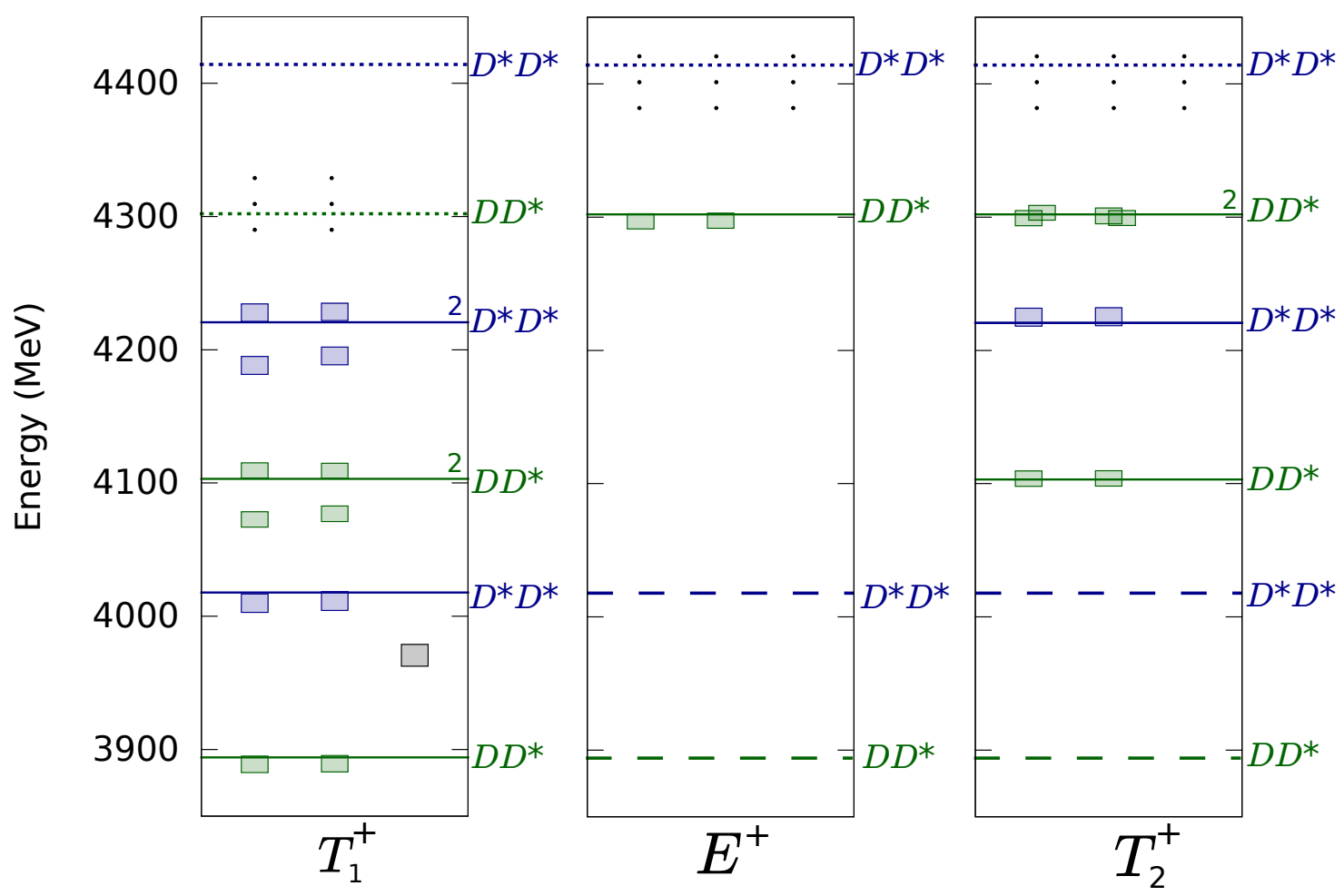

Figure 26: Figure was taken from Ref. [697]. The spectrum plot for the isospin-0 doubly-charmed sector with quark flavor content $c c \bar{l}$. The three columns of boxes and the horizontal lines are the same as in Fig. 24.

candidate for $X(5568)$ with $J^{P}=0^{+}$.

In Ref. [727], the authors studied the low-lying spectrum of the $b b \bar{b} \bar{b}$ system using the lattice nonrelativistic QCD methodology to search for a stable tetraquark state below the corresponding lowest noninteracting bottomonium-pair threshold. They constructed a full $S$-wave basis for the $1_{c} \times 1_{c}, 8_{c} \times 8_{c}$ meson-meson type of interpolating operators and the $\overline{3}_{c} \times 3_{c}, 6_{c} \times \overline{6}_{c}$ diquark-antidiquark type of operators with quantum numbers $J^{P C}=0^{++}$coupling to $2 \eta_{b}$ and $2 \Upsilon$, the $J^{P C}=1^{+-}$coupling to the $\eta_{b} \Upsilon$ and $J^{P C}=2^{++}$coupling to $2 \Upsilon$. They employed four gluon field ensembles at lattice spacings ranging from $a=0.06-0.12 \mathrm{fm}$, and one ensemble which had physical light-quark masses. All ensembles had $u, d, s$ and $c$ quarks in the sea. The results showed no evidence of a stable tetraquark candidate below the noninteracting bottomonium-pair thresholds in any channel by studying a full $S$-wave color-spin basis of QCD operators. To ensure the robustness of this conclusion, they added an auxiliary scalar potential into the QCD interactions with the objective of pushing a near threshold tetraquark increasingly lower than the threshold. As a result, they found no indication of any state below the noninteracting $2 \eta_{b}$ threshold in the $0^{++}, 1^{+-}$or $2^{++}$channel.

\subsection{Pentaquarks}

The charmonium-nucleon scattering at low energy has been studied in lattice QCD before the LHCb's observation of the hidden-charm pentaquark states. In Ref. [728], Kawanai and Sasaki studied the charmonium-nucleon potentials for both the $\eta_{c}-N$ and $J / \psi-N$ systems in quenched lattice QCD. The central and spin-independent potentials were calculated from the equal-time BS amplitude through the effective Schrödinger equation. They performed the quenched lattice QCD simulations on two different lattice sizes with a lattice cutoff of $a^{-1} \approx 2.1 \mathrm{GeV}$. They used nonperturbatively $\mathcal{O}(a)$ improved Wilson fermions for light quarks [729] and a relativistic heavy quark (RHQ) action for the 

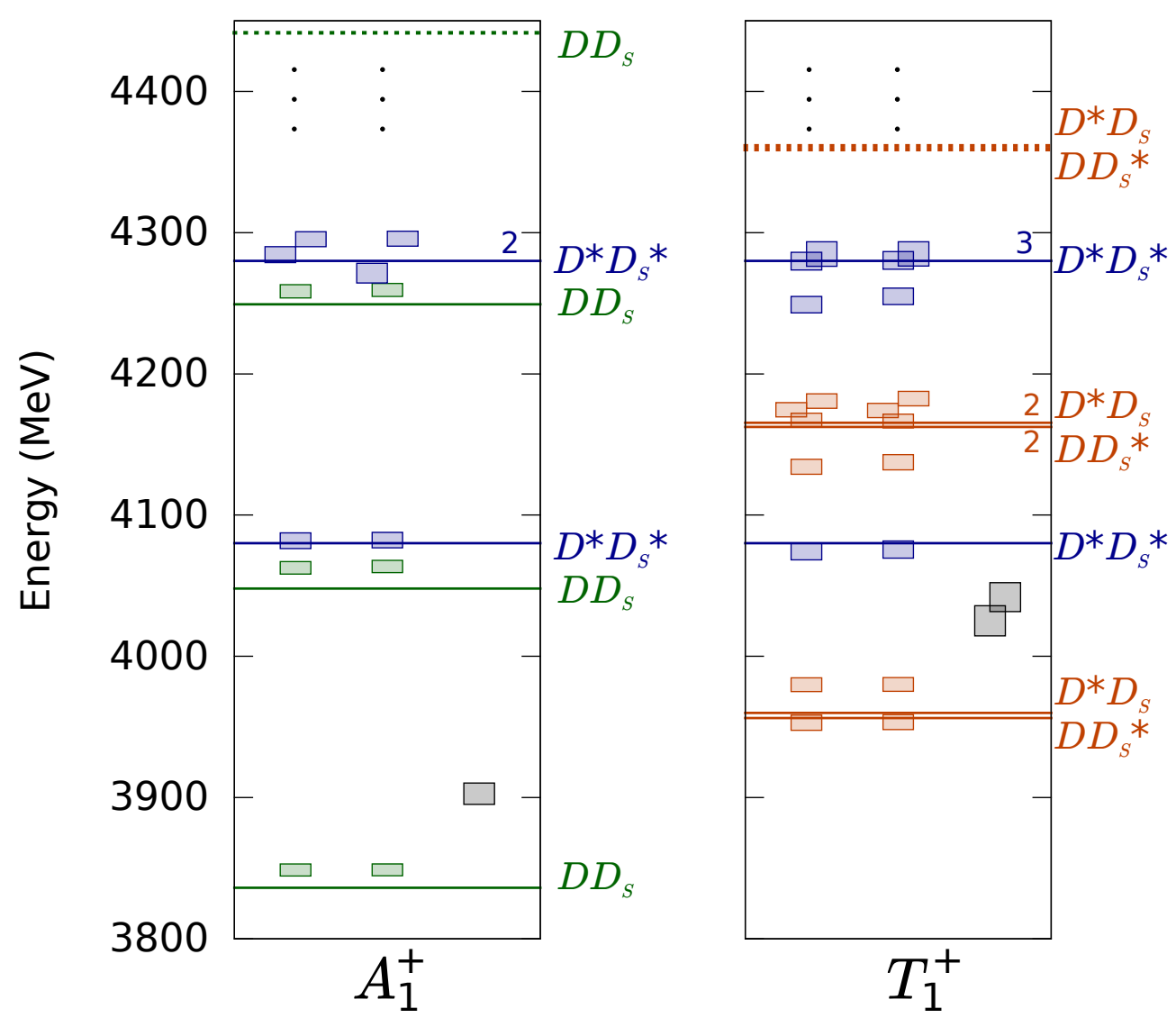

Figure 27: As Fig. 26 but for the isospin- $\frac{1}{2}$ doubly-charmed sector with quark flavor content $c c \bar{l} \bar{s}$. Figure was taken from Ref. [697].

charm quark [730]. As a result, the authors found that the all charmonium-nucleon potentials were weakly attractive at short distances at $m_{\pi}=640 \mathrm{MeV}$, while the attractive interaction in the $J / \psi-N$ systems were rather stronger than that of the $\eta_{c}-N$ system. However, these potentials were still not strong enough to form a bound state in the $J / \psi-N$ system. Moreover, the attractive interaction in the $\eta_{c}-N$ system tends to get slightly weaker as the light quark mass decreases, which may imply that the strength of the $\eta_{c}-N$ potential at the physical point becomes much weaker than that measured at $m_{\pi}=640 \mathrm{MeV}$. Similar conclusions were also obtained previously in the preliminary quenched lattice QCD study of the $\eta_{c}-N$ and $J / \psi-N$ scattering for $m_{\pi}=293-598 \mathrm{MeV}$ [731], and in the computation of the scattering lengths of the charmonium scattering with light hadrons in full QCD at $m_{\pi} \approx 197$ $\mathrm{MeV}$ [732]. However, the NPL Collaboration reported a contradictory result in Ref. [733] that the $\eta_{c}-N$ system with $J^{P}=\frac{1}{2}^{-}$has a deeply bound state with binding energy of $19.8(2.6) \mathrm{MeV}$, by using lattice QCD calculations at very heavy pion mass $m_{\pi}=805 \mathrm{MeV}$ and a single lattice spacing $b=0.145(2) \mathrm{fm}$.

To further discriminate the conflicting results between Refs. [728] and [733], Sugiura et. al. performed the time-dependent HAL QCD method to study the charmonium-nucleon effective central interactions in Ref. [734]. The authors focused on three $S$-wave channels $\eta_{c} N\left(J^{P}=\frac{1}{2}^{-}\right), J / \psi N\left(J^{P}=\frac{1}{2}^{-}\right)$, and $J / \psi N\left(J^{P}=\frac{3}{2}^{-}\right)$, which were a part of the coupled channels that can couple to the hidden-charm pentaquark states. The calculations were employed with $2+1$ flavor full QCD gauge configurations at the lattice spacing $a=0.1209 \mathrm{fm}$. The clover action was used for all quarks including charm quarks. They calculated the effective central potentials in the two $J / \psi N$ and one $\eta_{c} N$ channels for different time slices. The scattering phase shift for $J / \psi N$ was also obtained by solving the $S$-wave radial Schrödinger equation. They found the attractive interactions for all channels, however, not strong enough to form 
bound states. This result confirmed the time-independent method observation in Ref. [728].

The first lattice simulation reaching the energy region of the hidden-charm pentaquark states $P_{c}(4380)$ and $P_{c}(4450)$ was performed in Ref. [735], in which both the $S$-wave and $P$-wave, both $\eta_{c} N$ and $J / \psi N$ systems were studied including all possible $J^{P}$ assignments. The authors considered the nucleon-charmonium systems with total momentum zero of the form $O \sim N(p) M(-p)$, where $N$ is nucleon and $M$ denotes $J / \psi$ or $\eta_{c}$. They performed the simulations on the $N_{f}=2$ ensemble with a lattice spacing $a=0.1239 \mathrm{fm}$ at the pion mass $m_{\pi}=266 \mathrm{MeV}$. The Wilson Clover action was adopted for all light quarks and charm quarks. The energy spectra were calculated in the one-channel approximation for all six irreps of the discrete lattice group $O_{h}$ : irrep $G_{1}^{ \pm}$with $J^{P}=\frac{1}{2}^{ \pm}, \frac{7}{2}^{ \pm}$, irrep $G_{2}^{ \pm}$with $J^{P}=\frac{5}{2}^{ \pm}, \frac{7}{2}^{ \pm}$, irrep $H^{ \pm}$with $J^{P}=\frac{3}{2}^{ \pm}, \frac{5}{2}^{ \pm}, \frac{7}{2}^{ \pm}$. The final eigenenergies were presented in Fig. 28 for the $J / \psi N$ system and in Fig. 29 for the $\eta_{c} N$ system. As seen in these plots, the extracted lattice energy spectra were exactly consistent with the prediction of the non-interacting nucleon-charmonium systems. No additional eigenstates were found, which implied that there was no strong indication for a hidden-charm pentaquark resonance in the one-channel approximation for $\eta_{c} N$ and $J / \psi N$ scatterings.

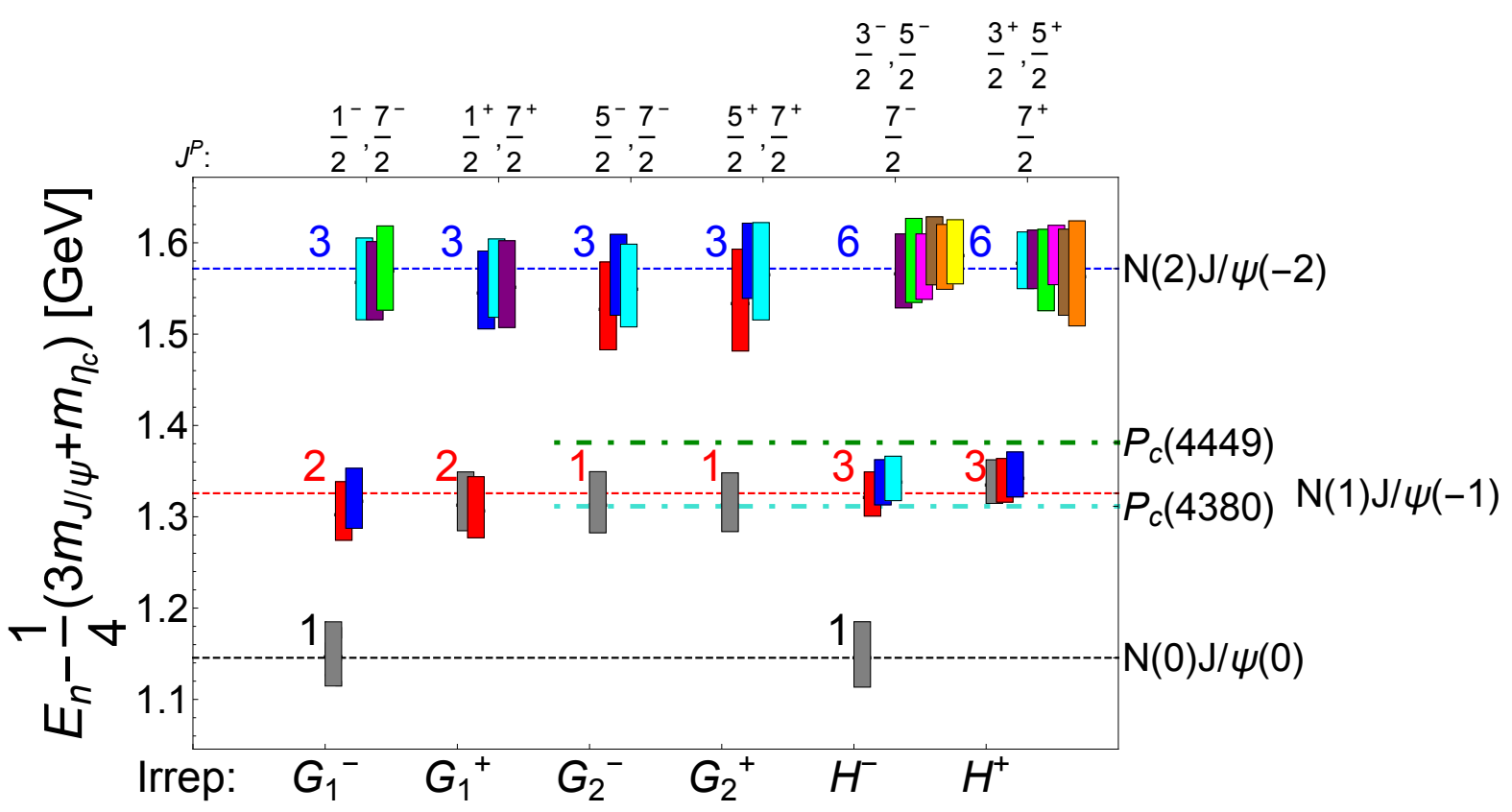

Figure 28: Eigenenergies of $J / \psi N$ system in one-channel approximation for all lattice irreps, denoted as the boxes. The numbers denote the degeneracy of the expected states in the non-interacting limit. Dashed lines represent the non-interacting energies $E_{N}(p)+E_{J / \psi}(-p)$ for different value of momentum $p$. The green and turquoise dash-dotted lines correspond to the experimental masses of $P_{c}(4380)$ and $P_{c}(4450)$. Figure was taken from Ref. [735].

The hadroquarkonium picture [736] was proposed for the modification of the potential between a static $c \bar{c}$ pair (in the heavy quark limit) induced by the presence of octet and decuplet light baryons. In Ref. [737], the authors performed lattice QCD simulations on a CLS ensemble with $N_{f}=2+1$ flavors of nonperturbatively improved Wilson quarks at a pion mass $m_{\pi} \approx 223 \mathrm{MeV}$ at a lattice spacing $a=0.0854 \mathrm{fm}$. The shift of the $c \bar{c}$ binding energies due to the presence of the nucleon was extracted to be down only by a few $\mathrm{MeV}$, similar in strength to the deuterium binding. It was dubious whether such a small attraction survives in the infinite volume limit and supports the existences of bound states or resonances. 


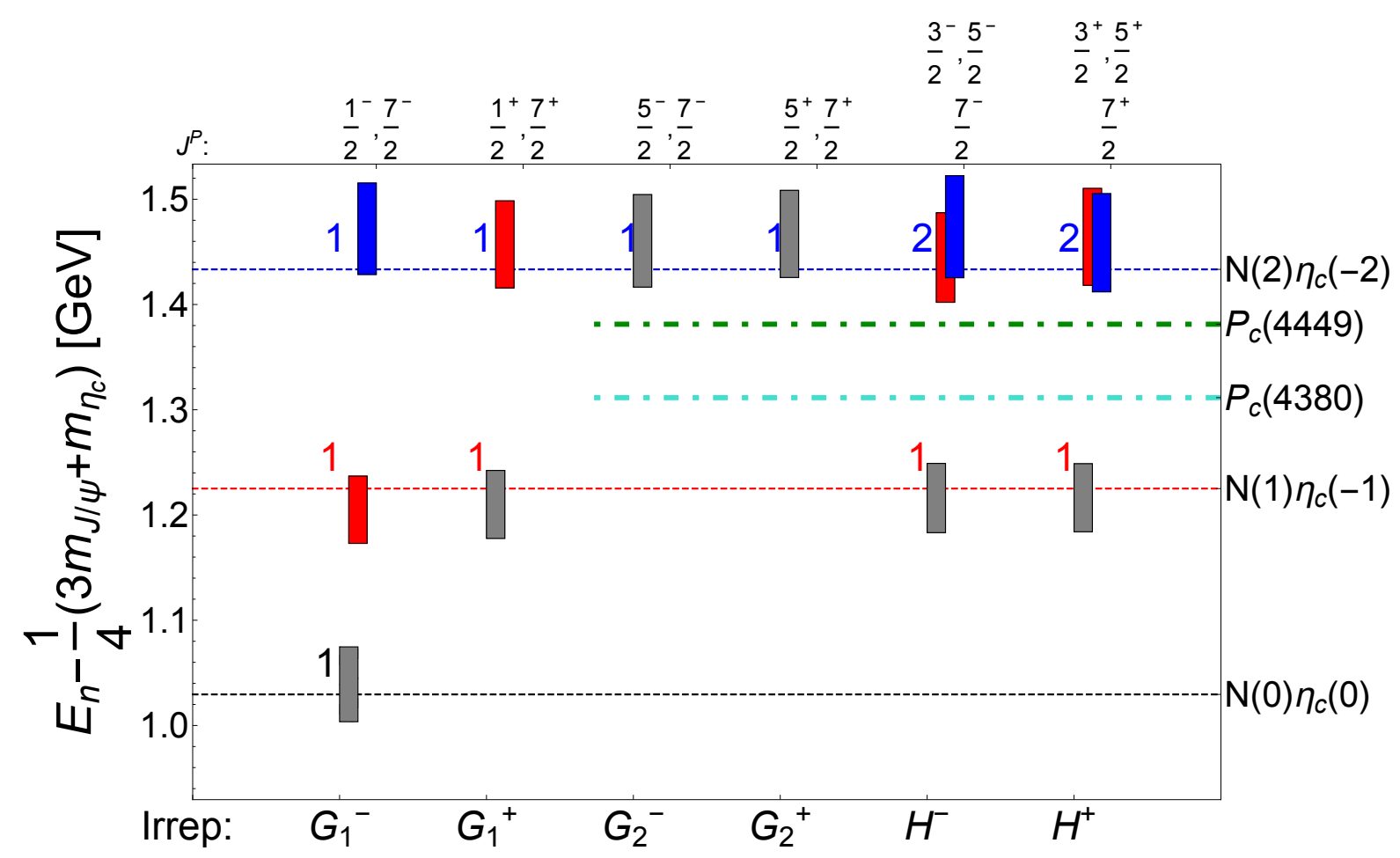

Figure 29: Eigenenergies of $\eta_{c} N$ system in one-channel approximation for all lattice irreps. Notations are the same with those in Fig. 28. Figure was taken from Ref. [735].

\subsection{A short summary for lattice QCD}

We summarize some important conclusions from the present lattice QCD investigations as follows:

- Lattice QCD simulations found an isosinglet $I=0$ candidate for the $X(3872)$ state close to the experimental data, only if both the conventional $c \bar{c}$ operators and the two-meson $D \bar{D}^{*}$ operators were used. However, they found no candidate if the diquark-antidiquark and $D \bar{D}^{*}$ interpolators were used in the absence of $c \bar{c}$, implying the $c \bar{c}$ Fock component is crucial for the $X(3872)$. The inclusion or exclusion of the diquark-antidiquark operators does not affect the lattice energy levels significantly.

- The lattice QCD simulations for the mass spectra of the hidden-charm four-quark systems didn't find any additional energy level in the $I^{G}\left(J^{P C}\right)=1^{+}\left(1^{+-}\right)$channel associated with the $Z_{c}(3900)$ and $Z_{c}(4020)$ states. The lattice investigations of the multiple channel scattering indicated that the $Z_{c}(3900)$ is not a conventional resonance but a threshold cusp.

- A quenched lattice QCD calculation showed that the interactions between the $\left(\bar{D}_{1} D^{*}\right)^{ \pm}$system are attractive for both the pseudoscalar $\left(S\right.$-wave) and axial-vector $\left(P\right.$-wave) channels. The $Z^{+}(4430)$ might be a candidate of the $P$-wave $\bar{D}_{1} D^{*}$ molecular state.

- The $1^{--}$hybrid charmonium mass from Lattice calculations is consistent with the experimental mass of the $Y(4260)$, which supported the hybrid interpretation for this state.

- Lots of lattice simulations showed that the $u d \bar{b} \bar{b}$ tetraquark state with quantum numbers $I\left(J^{P}\right)=$ $0\left(1^{+}\right)$lies below the $B B^{(*)}$ thresholds, implying that this state is stable against the strong and electromagnetic interaction. This observation has been confirmed at the sufficiently low pion masses $m_{\pi} \approx 164 \mathrm{MeV}$. 
- There is no evidence of a $b b \bar{b} \bar{b}$ tetraquark state with a mass below the lowest noninteracting $2 \eta_{b}$ threshold in the $0^{++}, 1^{+-}$or $2^{++}$channel in the lattice nonrelativistic QCD studies.

- To date, there are few lattice studies for many other $X Y Z$ structures reported in the last 16 years. Future investigations are badly needed to study these claimed signals in various channels.

- Most of the present lattice simulations were performed with a rather large pion mass. The pion exchange force plays a pivotal role in the formation of the loosely bound molecular states, which decays exponentially with $m_{\pi}$ and is very sensitive to the pion mass. Full QCD simulations near the physical point are crucial to explore the underlying structures of the near-threshold $X Y Z$ states.

- The lattice studies of the $\eta_{c} N$ and $J / \psi N$ scatterings in the one-channel approximation found no strong indication for the existence of $P_{c}(4380)$ and $P_{c}(4450)$ pentaquark states. The strong coupling between the $J / \psi N$ with other two-hadron channels might be important for the formation of the $P_{c}$ states. The multiple channel scattering need to be investigated in the future lattice simulations to confirm or refute the existence of the $P_{c}$ states.

\section{Production and decay properties}

In this subsection, we shall discuss recent theoretical progress on the productions and decay properties of the multiquark states, which are crucial to understand the nature of exotic states. In particular, the non-resonant explanations for some $X Y Z$ states depend on their production processes.

The production of the multiquark systems at the quark level remains very challenging since nonperturbative effects are usually involved. One has to turn to effective formalisms such as the nonrelativistic QCD (NRQCD) framework [738, 162, 161, 739, 740] and quark combination models [741]. At the hadron level, one may check whether it is necessary to introduce new resonances through refitting the production cross sections and line shapes. There are investigations of the interference effects on line shapes, $R$ values, and resonance properties due to the nearby hadrons. There are also explorations of amplitude pole structures in understanding the nature of resonances by considering the rescattering or meson loop mechanism. An interesting observation is that the anomalous triangle singularity (ATS) in final state interactions through the triangle diagram may mimic resonance-like structures in the mass invariant distributions of measured channels, when all the three particles in the loop approach their on-shell conditions simultaneously. If this mechanism were confirmed, the number of genuine resonances might be reduced. In the literature, there are also discussions about productions of exotic hadrons in heavy ion collisions [742, 743, 744].

One may adopt the widely used Cornell model or ${ }^{3} P_{0}$ pair creation model to study the decays of higher charmonium(like) states [745, 746]. The quark-interchange model was proposed for the rearrangement decays of the multiquark states. [747, 748, 749, 750]. The rescattering mechanism may be used to study the decays at the hadron level [751].

\section{$10.1 Q \bar{Q} q \bar{q}$}

\subsubsection{The $X$ states}

In Ref. [752], Zhou, Xiao, and Zhou reanalyzed the $\gamma \gamma \rightarrow D \bar{D}$ and $\gamma \gamma \rightarrow J / \psi \omega$ processes where the $X(3930)$ [now called $\chi_{c 2}(3930)$ ] and the $X(3915)$ were observed, respectively. Their results indicate that these two mesons are the same $2^{++}$state. In Ref. [753], Baru, Hanhart, and Nefediev investigated whether the $X(3915)$ is indeed the tensor partner of the $X(3872)$ in the molecule picture [754] by analysing the production amplitudes. They found that current data favor a scalar assignment, if the 
$X(3915)$ is a $D^{*} \bar{D}^{*}$ molecule. Its nature would require some exotic interpretation (i.e., neither a regular quarkonium state nor the $D^{*} \bar{D}^{*}$ spin partner of the $X(3872)$ ), if it is indeed dominated by the helicity-0 contribution of the nearby tensor state.

The hadronic effects on the $X(3872)$ meson abundance in heavy ion collisions were studied in Ref. [755], where the authors found that the absorption cross sections and the time evolution of the $X(3872)$ meson abundance were strongly dependent on its structure and quantum numbers. Combining the double parton scattering and basic ideas of the color evaporation model, the authors of Ref. [756] developed a model for the tetraquark production. They predicted the $X(3872)$ production cross section at $\mathrm{LHC} \sqrt{s}=14 \mathrm{TeV}$ to be in the order of tens of nb. In Ref. [757], Wang and Zhao discussed dependence of the ratio between the $B_{c}$ semileptonic and nonleptonic decays into the $X(3872)$. They demonstrated that the ratios would be universal and predictable if the $X(3872)$ production mechanism is through the $c \bar{c}$ component, while significant deviations from the predicted results would be a clear signal for the non-standard charmonium structure at the short distance.

In Ref. [758], the authors emphasized the connection between the decays $\Lambda_{b} \rightarrow X_{c}^{0} \Lambda$ and $B^{-} \rightarrow$ $X_{c}^{0} K^{-}$, with $X_{c}^{0}=c \bar{c} q \bar{q}(q=u, d, s)$ since these processes together with the case $X_{c}^{0} \rightarrow J / \psi$ arise from the $b \rightarrow c \bar{c} s$ transition at the quark level. Assuming the $X(3872)$ and $X(4140)$ to be tetraquark states, Hsiao and Geng predicted the branching ratios of $\Lambda_{b} \rightarrow \Lambda X(3872) \rightarrow \Lambda J / \psi \pi^{+} \pi^{-}$and $\Lambda_{b} \rightarrow$ $\Lambda X(4140) \rightarrow \Lambda J / \psi \phi$ are $(5.2 \pm 1.8) \times 10^{-6}$ and $(4.7 \pm 2.6) \times 10^{-6}$, respectively, which were expected to be accessible at LHCb. In Ref. [759], they predicted the branching fractions for $B^{-} \rightarrow X_{c}^{0} \pi^{-}$, $\bar{B}^{0} \rightarrow X_{c}^{0} \bar{K}^{0}, \bar{B}_{s}^{0} \rightarrow X_{c}^{0} \bar{K}^{0}, B_{c}^{-} \rightarrow X_{c}^{0} \pi^{-}, B_{c}^{-} \rightarrow X_{c}^{0} K^{-}, B_{c}^{-} \rightarrow J / \psi \mu^{-} \bar{\nu}_{\mu}$, and $B_{c}^{-} \rightarrow X_{c}^{0} \mu^{-} \bar{\nu}_{\mu}$.

In Ref. [760], productions of the $X(3940)$ and $X(4160)$ in exclusive weak decays of $B_{c}$ were studied with the improved Bethe-Salpeter method by assigning these two mesons as $\eta_{c}(3 S)$ and $\eta_{c}(4 S)$, respectively. The predicted branching ratios are $\operatorname{Br}\left(B_{c}^{+} \rightarrow X(3940) e^{+} \nu_{e}\right)=1.0 \times 10^{-4}$ and $\operatorname{Br}\left(B_{c}^{+} \rightarrow\right.$ $\left.X(4160) e^{+} \nu_{e}\right)=2.4 \times 10^{-5}$. Nonleptonic $B_{c}$ decays to these states were also investigated. In Ref. [761], the same group investigated the two-body open-charm OZI-allowed strong decays of these two mesons with the ${ }^{3} P_{0}$ model. The decay width of $\eta_{c}(3 S)$ is around $34 \mathrm{MeV}$ and close to the width of $X(3940)$. Therefore, the $\eta_{c}(3 S)$ is a good candidate of $X(3940)$. Although the width of $\eta_{c}(4 S)$ is around $70 \mathrm{MeV}$ and close to the lower limit of the width of $X(4160)$, the ratio $\Gamma\left(D \bar{D}^{*}\right) / \Gamma\left(D^{*} \bar{D}^{*}\right)$ of $\eta_{c}(4 S)$ is larger than the experimental data of $X(4160)$. The authors concluded that $\eta_{c}(4 S)$ is not the candidate of $X(4160)$.

In Ref. [270], Liu tried to understand the nature of $X(4140), X(4274), X(4500)$, and $X(4700)$ in the process $B^{+} \rightarrow J / \psi \phi K^{+}$with the rescattering mechanism. The $X(4700)$ and $X(4140)$ may be simulated by the rescattering effects, but the $X(4274)$ and $X(4500)$ could not if the quantum numbers of $X(4274)$ and $X(4500)$ are $1^{++}$and $0^{++}$, respectively, which indicates that the $X(4274)$ and $X(4500)$ could be genuine resonances. The arguments from the mass, decay, and production [33, 269, 762, 60, 59] lead to the suggestion that the $X(4274)$ may be the conventional $\chi_{c 1}(3 P)$. In Ref. [763], Liu and Oka investigated the radiative transition processes $e^{+} e^{-} \rightarrow \gamma J / \psi \phi, \gamma J / \psi \omega$, and $\pi^{0} J / \psi \eta$ to search for the $c \bar{c} s \bar{s}$ states, such as the $X(4140), X(4274), X(4350)$, and $X(3915)$, by considering the rescattering processes via the charmed-strange meson loops. They found that the anomalous triangle singularity (ATS) may appear as narrow peaks, when some special conditions are satisfied, and thus nonresonance explanation is possible for their structures. Contrary to genuine resonances, the location of the ATS peaks depends on kinematic configurations, and their movements may be used to distinguish the ATS peaks from genuine resonances, once high resolution experiments are available.

In Ref. [764], the production of $X(3872)$ as a $D^{*} \bar{D}$ molecule was considered in the process $\bar{D}^{(*)} D^{(*)} \rightarrow$ $\pi X(3872)$ by using the heavy meson effective theory. In Ref. [765], the $X(3872)$ production and absorption in the hot hadron gas produced in heavy ion collisions were studied. The authors found that the average $X(3872)$ number as a $D \bar{D}^{*}$ molecule, $N_{X(\mathrm{~mol})} \approx 7.8 \times 10^{-4}$, is about 80 times larger than that as a tetraquark state, since the production mechanisms are quite different. Similar investigations about the $Z_{b}(10610)$ and $Z_{b}(10650)$ cases can be found in Refs. [766, 767, 768]. 
The authors of Ref. [769] studied the production and decay of the exotic hadrons in multiproduction processes at high energy hadron colliders in the non-relativistic wave function framework by treating the exotic states as hadronic molecules. The rapidity and transverse momentum distributions of the $X(3872), Y(4260)$, and $P_{c}(4380)$ at $\sqrt{s}=8 \mathrm{TeV}$ in $p p$ collisions were investigated.

The authors of Ref. [770] discussed the possibility of the $X(4140)$ being the $\chi_{c 1}(3 P)$ state through the $\chi_{c 1} \pi^{+} \pi^{-}$invariant mass spectrum in the $B \rightarrow K \chi_{c 1} \pi^{+} \pi^{-}$process. The analysis for the $D \bar{D}$ invariant mass in the $B \rightarrow K D \bar{D}$ decay results in a $\chi_{c 0}(3 P)$ candidate with a mass around $4080 \mathrm{MeV}$. From the decay behaviors of the $\chi_{c J}(3 P)$, the assignments of the $X(4140)$ and $X(4080)$ as the $\chi_{c 1}(3 P)$ and $\chi_{c 0}(3 P)$ are reinforced respectively.

In Ref. [771], Kang and Oller introduced a near-threshold parameterization to analyze the $X(3872)$ production processes $p \bar{p} \rightarrow J / \psi \pi \pi+\ldots, B \rightarrow K J / \psi \pi \pi$, and $B \rightarrow K D \bar{D}^{* 0}$. The data can be reproduced with similar quality for $X(3872)$ being a bound and/or a virtual state. The authors of Ref. [772] studied the $J / \psi \phi$ mass distribution of the $B^{+} \rightarrow J / \psi \phi K^{+}$reaction, and found that the contributions of the narrow $X(4140)$ and the $X(4160)$ are needed, which provided an explanation for the large width of $X(4140)$ observed by LHCb $[59,60]$. The $X(4160)$ state was found to be strongly tied to the $D_{s}^{*+} D_{s}^{*-}$ channel.

In Ref. [773], Dai, Dias, and Oset studied the $B_{c}^{-} \rightarrow \pi^{-} J / \psi \omega$ and $B_{c}^{-} \rightarrow \pi^{-} D^{*} \bar{D}^{*}$ reactions. They found that the molecular states $X(3940)$ and $X(3930)$ couple mostly to $D^{*} \bar{D}^{*}$ in the $2^{++}$and $0^{++}$ channels respectively, and have big influence on the $J / \psi \omega$ mass distribution. Later in Ref. [774], the authors studied the semileptonic decay of the $B_{c}^{-}$meson into the $2^{++} X(3930), 0^{++} X(3940)$, and $2^{++}$ $X(4160)$ resonances by treating them as dynamically generated states.

In Ref. [775], the productions of the $X(4350) \rightarrow \phi J / \psi$ and $X(3915) \rightarrow \omega J / \psi$ in $\gamma \gamma$ interactions in $p p / p P b / P b P b$ collisions at the LHC were investigated. The results with both $J^{P C}=0^{++}$and $2^{++}$ indicate that the experimental study is feasible and can be used to check the existence of these two states. In Refs. [776, 777], Braaten, He, and Ingles pointed out that the production rate of $X(3872)$ accompanied by a pion at a high energy hadron collider should be larger than that of $X(3872)$ without a pion, if the $X(3872)$ is a weakly bound charm-meson molecule. This type of production may happen through the creation of the $J=1 D^{*} \bar{D}^{*}$ pair at short distances followed by a rescattering process. In Ref. [778], the production of $X(3872)$ accompanied by a pion in $B$ meson decays was discussed. Such a production should be observable, which can be used to support for the interpretation of $X(3872)$ as a molecule.

In Ref. [779], the transverse momentum spectra and yields of the $X(3872), J / \psi$, and $\psi(2 S)$ for heavyion collisions at the LHC energies are predicted within the framework of the statistical hadronization model. The production yield of $X(3872)$ was found to be about $1 \%$ relative to that for $J / \psi$. In Ref. [780], Guo proposed a method to study whether the $X(3872)$ is above or below the $D^{0} \bar{D}^{* 0}$ threshold. He found that the line shape of $X(3872) \gamma$ is very sensitive to the deviation of the $X(3872)$ mass from the threshold due to a triangle singularity. This indirect method can be applied to experiments producing copious $D^{* 0} \bar{D}^{* 0}$ pairs.

In Ref. [781], the production of $1^{++} X_{b}=B \bar{B}^{*}$ molecule in the process $\Upsilon(5 S, 6 S) \rightarrow \gamma X_{b}$ was investigated by using the rescattering mechanism. The production ratios are orders of $10^{-5}$. In Ref. [782], a comparative study for the strong decays of $X(3915)$ as a $0^{++}$charmonium state into $D \bar{D}$ and $J / \psi \omega$ channels were performed with the Cornell potential model, and a generalized screen potential model. The suppression of the $D \bar{D}$ mode can be understood with both models, while the significant $J / \psi \omega$ mode favors the latter model.

In Ref. [334], the decay $X(3872) \rightarrow J / \psi \gamma$ was investigated in the Bethe-Salpeter equation approach if it is a $D \bar{D}^{*}$ molecule. The decay width was found to be in the range $8.7 \sim 49.5 \mathrm{keV}$. The authors of Ref. [783] studied the two-photon decay of the $X(3915)$ state if it as a $D^{* 0} \bar{D}^{* 0}$ molecule. The obtained decay width is $36 \mathrm{keV}$. The decay width into $\mathrm{J} / \psi \omega$ is $66 \mathrm{MeV}$ in the same molecular picture [784]. 


\subsubsection{The $Y$ states}

In Ref. [785], in the Fano-like interference scenario, Chen, Liu, Li, and Ke studied the asymmetric line shapes of the $Y(4260)$ and $Y(4360)$ structures in their discovery processes $e^{+} e^{-} \rightarrow \pi \pi J / \psi$ and $e^{+} e^{-} \rightarrow \pi \pi \psi(3686)$ by considering the interference between continuum and resonance contributions. They found that the broad structure $Y(4008)$ can be induced by the Fano-like interference. Their numerical results indicate that the $Y(4008), Y(4260)$, and $Y(4360)$ may be not genuine resonances, which may explain the absence of these states in the $R$ value scan and the non-observation of their open-charm decay modes. The Fano-like phenomena were also expected in processes such as $e^{+} e^{-} \rightarrow$ $\pi \pi \psi(3770)$ and $e^{+} e^{-} \rightarrow K \bar{K} J / \psi$. In Ref. [786], Chen, Liu, and Matsuki studied the interference effects in understanding the $Y(4320)$ and $Y(4390)$. They found that the signals of these two structures can be reproduced by introducing the interference effects between $\psi(4160), \psi(4415)$, and background contributions. Therefore, the $Y(4320)$ and $Y(4390)$ may be not genuine resonances. A better description of experimental results needs the introduction of $Y(4220)$, which was proposed to be the charmonium $\psi(4 S)$. In Ref. [787], the updated data of $Y(4220)$ were used to categorize this state into the $J / \psi$ family in a $4 S-3 D$ mixing scheme. The present experimental data seem to support the charmonium assignment. The assignments of higher charmonia states and properties of newly predicted states were also discussed.

In Ref. [745], the open-charm decay mode $\Lambda_{c} \bar{\Lambda}_{c}$ of $Y(4630)$ was explored in the ${ }^{3} P_{0}$ pair creation model by assuming this exotic meson to be a $P$-wave diquark-antidiquark state [97]. The decay mechanisms of $Y(4630)$ in both tetraquark and molecule pictures were studied in Ref. [788]. The $p \bar{p}, \pi \pi$, and $K \bar{K}$ decay modes of $Y(4630)$ were discussed in the $\Lambda_{c} \bar{\Lambda}_{c}$ rescattering mechanism in Ref. [751].

In Ref. [789], the role of the s-channel $Y(4630)$ in the $p \bar{p} \rightarrow \Lambda_{c} \bar{\Lambda}_{c}$ reaction near threshold was investigated. The authors found that this state gives a clear bump structure with the magnitude of $10 \mu \mathrm{b}$, which can be tested by the PANDA experiment. In Ref. [790], productions of $Y(4220)$ in the processes $e^{+} e^{-} \rightarrow Y(4220) \rightarrow p \bar{p} \pi^{0}$ and $p \bar{p} \rightarrow Y(4220) \pi^{0}$ were studied simultaneously, where the nucleon and its excitations play an important role in the reactions. Therefore, the PANDA experiment can also be used to study the properties of $Y(4220)$.

In Ref. [260], Qin, Xue, and Zhao investigated the production mechanism of $Y(4260)$ in $e^{+} e^{-}$ annihilation by assuming it to be a $\bar{D} D_{1}+$ c.c. molecule admixed with a compact $c \bar{c}$ component. They found that the heavy quark spin symmetry breaking plays a crucial role for the $Y(4260)$ production, and the molecule picture can describe the available observables simultaneously. They also found that $\bar{D} D^{*} \pi+$ c.c. is one of the important decay channels of $Y(4260)$. In Ref. [791], Cleven and Zhao demonstrated that the molecule picture of $Y(4260)$ can explain the cross section line shape of $e^{+} e^{-} \rightarrow$ $\chi_{c 0} \omega$ around the $Y(4260)$ mass region. Further in Ref. [792], Xue, Jing, Guo, and Zhao showed that the partial widths of the molecular $Y(4260)$ to $D^{*} \bar{D}^{*}$ and $D_{s}^{*} \bar{D}_{s}^{*}$ are much smaller than the width to $\bar{D} D^{*} \pi+$ c.c., and the $Y(4260)$ contributions to the cross section line shapes of the $e^{+} e^{-} \rightarrow D^{*} \bar{D}^{*}$ and $D_{s}^{*} \bar{D}_{s}^{*}$ are rather small in most of the energy region from thresholds to about $4.6 \mathrm{GeV}$. They also found that the interferences of the $Y(4260)$ with nearby charmonium states $\psi(4040), \psi(4160)$, and $\psi(4415)$ produce a dip around $4.22 \mathrm{GeV}$ in the $e^{+} e^{-} \rightarrow D^{*} \bar{D}^{*}$ cross section line shape.

In Ref. [793], Dai, Haidenbauer, and Meissner investigated the reaction $e^{+} e^{-} \rightarrow \Lambda_{c}^{+} \bar{\Lambda}_{c}^{-}$at energies close to the threshold after considering the effects from the $Y(4630)$ resonance and the final state interactions. The mass and width of this state were found to be around $4652 \mathrm{MeV}$ and $63 \mathrm{MeV}$, respectively, and thus the $Y(4630)$ and $Y(4660)$ could be the same state. In Ref. [794], a combined fit was performed to the cross sections of $e^{+} e^{-} \rightarrow \omega \chi_{c 0}, \pi \pi h_{c}, \pi \pi J / \psi, \pi \pi \psi(3686)$, and $\pi^{+} D^{0} D^{*-}+c . c$. with three resonances $Y(4220), Y(4390)$, and $Y(4660)$. The authors found that the first two resonances are sufficient to explain these cross sections below $4.6 \mathrm{GeV}$, so the $Y(4320), Y(4360)$, and $Y(4390)$ should be one state.

In Ref. [795], the $D \bar{D}^{*}$ loop contributions to the $e^{+} e^{-}$reaction around $4.0 \mathrm{GeV}$ were considered with 
effective Lagrangians. The authors noticed a second pole dynamically generated by the meson-meson quantum fluctuations in addition to the charmonium $\psi(4040)$. This pole leads to the controversial state $Y(4008)$ observed in $e^{+} e^{-} \rightarrow \pi \pi J / \psi$. The authors also expect that the pole could be visible in the process $e^{+} e^{-} \rightarrow \psi(4040) \rightarrow D \bar{D}^{*}$. The same mechanism may be applied to the $Y(4260)$ and $\psi(4160)$.

In Ref. [796], the relation between the $\psi(4160)$ and $Y(4260)$ within a unitarized effective Lagrangian approach was studied. The authors found that the $Y(4260)$ is not an independent resonance but a manifestation of the $\psi(4160)$ due to the final state interactions between the $D_{s}^{*} \bar{D}_{s}^{*}$ and the $J / \psi f_{0}(980)$. In Ref. [797], Chen, Dai, Guo, and Kubis studied the processes $e^{-} e^{+} \rightarrow Y(4260) \rightarrow J / \psi \pi \pi(K \bar{K})$ in the dispersion theory, and analyzed the roles of the light-quark $S U(3)$ singlet and octet states in the transitions. According to their investigations, the $Y(4260)$ contains a large light-quark component, and it is neither a hybrid nor a conventional charmonium state. The analysis of the ratio of the octet and singlet components indicates that the $Y(4260)$ has a sizeable $\bar{D} D_{1}$ component, although it is not a pure $\bar{D} D_{1}$ molecule.

Very recently, BESIII studied the reaction $e^{+} e^{-} \rightarrow \pi^{+} \pi^{-} D \bar{D}$ at center-of-mass energies above 4.08 $\mathrm{GeV}$, and observed $\psi(3770)$ and $D_{1}(2420) \bar{D}+$ c.c. in this process. Neither fast rise of the cross section above the $D_{1}(2420) \bar{D}$ threshold nor obvious structure is visible from the measurements, which is different from the expectation of Ref. [798].

In Ref. [799], Chen et al studied the decay of $Y(4260)$ into $Z_{c}(3900) \pi$ by assuming that they are $D \bar{D}_{1}$ and $D \bar{D}^{*}$ molecules, respectively. The partial width was found to be around $1.2 \sim 3.0 \mathrm{MeV}$. In Ref. [800], the open-charm decays of the charmonium $\psi\left(3^{3} D_{1}\right)$ were studied in the ${ }^{3} P_{0}$ pair creation model. The total width is consistent with those of both $Y(4320)$ and $Y(4390)$. One of the dominant decays of $Y(4390)$ is $Y(4390) \rightarrow D_{1} \bar{D} \rightarrow \pi^{+} D^{0} D^{*-}$.

In Ref. [746], Xiao et al. studied the $\Lambda_{c} \bar{\Lambda}_{c}$ decay mode of the higher vector charmonium states around $4.6 \mathrm{GeV}$ in the ${ }^{3} P_{0}$ pair creation model. They found that the partial decay widths for $S$-wave charmonia are about a few $\mathrm{MeV}$, and those for $D$-wave charmonia are less than one $\mathrm{MeV}$. The $Y(4660)$ is very likely to be an $S$-wave charmonium, if the $Y(4630)$ and $Y(4660)$ are the same state.

\subsubsection{The $Z_{c}$ states}

In Ref. [801], the final state interaction effects on the $Z_{c}(3900)$ was studied. In the analysis, the authors calculated the amplitudes of the $e^{+} e^{-} \rightarrow J / \psi \pi \pi, h_{c} \pi \pi$, and $D \bar{D}^{*} \pi$ processes, and searched poles in the complex energy plane. They found only one pole in the nearby energy region in different Riemann sheets and concluded that the $Z_{c}(3900)$ is a molecular state according to the pole counting rule $[802,803]$. In Ref. [804], the same group noted that the triangle singularity mechanism did not produce the observed $Z_{c}(3900)$ peak. Their study again favors the molecular assignment for the $Z_{c}(3900)$.

In Ref. [805], to understand the nature of $Z_{c}(3900)$, the authors considered the $J / \psi \pi$ and $D^{*} \bar{D}$ coupled-channel T-matrix analysis in a finite volume. Comparing their calculations with the lattice results [686], they concluded that it is difficult to distinguish whether the $Z_{c}(3900)$ is a resonance originating from a pole above the $D^{*} \bar{D}$ threshold or a virtual state below the threshold. In Ref. [806], the authors carried out a thorough amplitude analysis of the $Z_{c}(3900)$ data and considered four different scenarios corresponding to the pure QCD states, virtual states, or purely kinematical enhancements. They concluded that it is not possible to distinguish between these scenarios at that time, which contradicted the conclusion of Ref. [804].

Both the $X(3872)$ and $Z_{c}(3900)$ were observed in the $e^{+} e^{-}$annihilations (into $\gamma X(3872)$ and $\pi Z_{c}(3900)$, respectively), but only the $X(3872)$ was observed in $B$ decay. In order to understand this puzzle, Yang, Wang, and Meissner studied the isospin amplitudes in the exclusive $B$ decay into $D^{*} \bar{D} K$ in Ref. [807]. Their analysis indicates that the production of the isovector $C=-D^{*} \bar{D}$ state is highly suppressed compared to the isospin singlet one, if both states are $D \bar{D}^{*}$ molecules. Their findings seem to support the molecular nature of $Z_{c}(3900)$. 
In Ref. [808], He and Chen investigated the $\pi J / \psi-\bar{D}^{*} D$ interaction in the $Y(4260)$ decay in a coupled-channel quasipotential Bethe-Salpeter equation approach in order to understand the origin of the $Z_{c}(3900)$ and $Z_{c}(3885)$. They found a virtual state around $3870 \mathrm{MeV}$, and concluded that the two $Z_{c}$ states are from the same virtual state. In Ref. [809], Zhao analyzed possible quantum numbers and various explanations of the $Z_{c}(4100)$ state via its production in the $B$ decay process. He found that the state can be either induced by final state interaction effects arising from an $S$-wave $D^{*} \bar{D}^{*}$ rescattering with $I^{G}\left(J^{P C}\right)=1^{-}\left(0^{++}\right)$, or it is a $P$-wave resonance with $I^{G}\left(J^{P C}\right)=1^{-}\left(1^{-+}\right)$arising from the $D^{*} \bar{D}^{*}$ interaction. The latter structure can be regarded as an excited state of $Z_{c}(4020)$.

In Ref. [810], Cao and Dai discussed possible $J^{P}$ assignments for the $Z_{c}(4100), Z_{1}(4050)$ [called $Z_{c}$ (4050) in PDG [35]], and $Z_{2}(4250)$ and proposed that the $Z_{c}(4100)$ observed in $\eta_{c} \pi$ by LHCb [67] and the $Z_{1}(4050)$ observed in $\chi_{c 1} \pi$ by Belle [811] correspond to the same state. They suggested that the $Z_{2}(4250)$ should be a $1^{+}$or $1^{-}$state, while the $Z_{c}(4100) / Z_{1}(4050)$ could be a $0^{+}$or $1^{-}$state.

In Ref. [812], the authors demonstrated that the $Z_{c}(4430)$ and $Z_{c}(4200)$ can be consistently interpreted as kinematical singularities from the triangle diagrams. They explained why the $Z_{c}(4200)$ contribution was observed in $\Lambda_{b} \rightarrow J / \psi p \pi$ but $Z_{c}(4430)$ was not. In Ref. [813], the $Z_{1}(4050)$ and $Z_{2}(4250)$ structures observed in $\bar{B}^{0} \rightarrow \chi_{c 1} K^{-} \pi^{+}$were also explained with the triangle singularities, and the $J^{P}$ was predicted to be $1^{-}\left(1^{+}\right.$or $\left.1^{-}\right)$for $Z_{1}(4050)\left(Z_{2}(4250)\right)$.

In Ref. [814], the production of $Z_{c}(3900)$ and $Z_{c}(4020)$ in $B_{c}$ decays was studied with an effective Lagrangian approach by considering meson loop contributions. The branching ratios of $B_{c}$ decays to $Z_{c}(3900) \pi$ and $Z_{c}(4020) \pi$ are around $10^{-4}$ and $10^{-7}$, respectively. In a light-front model, Ke and Li studied the decays of $Z_{c}(3900)$ and $Z_{c}(4020)$ [called $X(4020)$ in [35]] into $h_{c} \pi$ by assuming them as $D \bar{D}^{*}$ and $D^{*} \bar{D}^{*}$ molecules, respectively [815]. They found that the $Z_{c}(4020)$ seems to be a molecular state. If the $Z_{c}(3900)$ is also a molecule, it would be observable in $e^{+} e^{-} \rightarrow h_{c} \pi$, since the partial width of $Z_{c}(3900)$ is only three times smaller than that of $Z_{c}(4020)$. The nonobservation of $Z_{c}(3900)$ in this channel with precise measurements would be helpful to rule out its molecule interpretation.

The role of the charged exotic states in the reaction $e^{+} e^{-} \rightarrow \psi(2 S) \pi^{+} \pi^{-}$was considered with a dispersive approach in Ref. [816]. The authors found that the $Z_{c}(3900)$ state plays an important role in explaining the BESIII's invariant mass distribution at both $\sqrt{s}=4.226$ and $4.258 \mathrm{GeV}$ [66]. The sharp narrow structure at $\sqrt{s}=4.416 \mathrm{GeV}$ can be explained with a heavier charged state whose mass and width are about $4.016 \mathrm{GeV}$ and $52 \mathrm{MeV}$, respectively. At $\sqrt{s}=4.358 \mathrm{GeV}$, no intermediate $Z_{c}$ state is necessary. The authors also concluded that the $\pi \pi$ final state interaction leads to the $\pi \pi$ invariant mass distribution for these four energies.

In Ref. [817], the strong decay widths of $Z_{c}(3900)$ into $J / \psi \pi$ and $\eta_{c} \rho$ were calculated with the coupling constants in the QCD sum rule method with the assumption that the $Z_{c}(3900)$ is a diquarkantidiquark state with $J^{P C}=1^{+-}$. The resulting decay width of these two modes is around $66 \mathrm{MeV}$, larger than the PDG value $28.2 \pm 2.6 \mathrm{MeV}$ [35]. In Ref. [818], the charmless decays of the $Z_{c}(3900)$ and $Z_{c}(4025)$ [called $X(4020)$ in [35]] into vector-pseudoscalar (VP) type light mesons were investigated with the rescattering mechanism by assuming these mesons to be $I^{G}\left(J^{P C}\right)=1^{+}\left(1^{+-}\right) D \bar{D}^{*}$ and $D^{*} \bar{D}^{*}$ molecules, respectively. The branching ratios of $Z_{c}(3900) \rightarrow V P\left(Z_{c}(4025) \rightarrow V P\right)$ are typically of the order $10^{-3}\left(10^{-5}\right)$.

In Ref. [819], the strong decay widths $Z_{c}(3900) \rightarrow J / \psi \pi\left(\eta_{c} \rho, \bar{D}^{0} D^{*+}, \bar{D}^{* 0} D^{+}\right)$and $Z(4430) \rightarrow J / \psi$ $(\psi(2 S) \pi)$ were calculated within a covariant quark model. The authors found that the tetraquark-type (molecule-type) current is in disaccord (accordance) with the experimental observation that $Z_{c}(3900)$ has a much stronger coupling to $D \bar{D}^{*} / D^{*} \bar{D}$ than to $J / \psi \pi$. They found that the $Z(4430)$ state is a good candidate for the compact tetraquark state, and predicted the partial decay width $\Gamma\left(Z_{4430}^{+} \rightarrow D^{*+}+\bar{D}^{* 0}\right)$ to be around $24 \mathrm{MeV}$.

In Ref. [750], Wang et al investigated the strong decays of $Z_{c}(3900) \sim D^{*} \bar{D}, Z_{c}(4020) \sim D^{*} \bar{D}^{*}$, $Z_{c}(4430) \sim \bar{D} D^{*}(2 S)$ or $\bar{D}^{*} D(2 S), Z_{b}(10610) \sim B^{*} \bar{B}$, and $Z_{b}(10650) \sim B^{*} \bar{B}^{*}$ into a heavy quarkonium plus a pion in a relativized quark model in the molecule picture. The decay proceeds through the 
interchange of a heavy quark and a light quark as well as the exchange of a gluon. Therefore, the exchanged $Q \bar{q}$ or $\bar{Q} q$ is a color-octet state. According to their results, the $Z_{c}(3900)$ and $Z_{c}(4020)$ have a larger coupling with $\psi(2 S) \pi$ than $J / \psi \pi$, but the partial width $\Gamma\left(Z_{c}(3900) \rightarrow J / \psi \pi\right)$ is much larger than $\Gamma\left(Z_{c}(3900) \rightarrow \psi(2 S) \pi\right)$. This feature is consistent with the experimental observations. The obtained decay ratios also favor the molecule assignments for the $Z_{b}$ states. However, the calculation does not favor the pure molecule assignment for the $Z_{c}(4430)$, which is different from the study with the naive nonrelativistic quark model [820].

In Ref. [821], Voloshin considered the decays $Z_{c}(4020) \rightarrow X(3872) \gamma$ and $Z_{c}(4020) \rightarrow X(3872) \pi$. He assumed that $X(3872)$ is dominantly an $S$-wave $D^{0} \bar{D}^{* 0}$ molecule and that the $Z_{c}(4020)$ is an $S$-wave $D^{*} \bar{D}^{*}$ resonance, where the transitions occur through $D^{*} \rightarrow D \gamma, D \pi$. The rates were found to be at most several tenths percent in terms of the branching fraction for the $Z_{c}(4020)$.

\subsubsection{The $Z_{b}$ states}

In Ref. [822], the role of the $Z_{b}$ states in the $\Upsilon(n S) \rightarrow \Upsilon(m S) \pi \pi(m<n \leq 3)$ transitions was analyzed by studying the final $\pi \pi$ rescattering effects with the dispersion theory. The $Z_{b}$ effects in $\Upsilon(2 S) \rightarrow \Upsilon(1 S) \pi \pi$ and $\Upsilon(3 S) \rightarrow \Upsilon(2 S) \pi^{0} \pi^{0}$ are very small, but those in $\Upsilon(3 S) \rightarrow \Upsilon(1 S) \pi \pi$ are significant. The inclusion of the $Z_{b}$ states and $\pi \pi$ interactions can explain the observed anomaly of $\Upsilon(3 S) \rightarrow \Upsilon \pi \pi$. Further in Ref. [823], the effects of the two intermediate $Z_{b}$ states and bottom meson loops on the dipion transition processes $\Upsilon(4 S) \rightarrow \Upsilon(1 S, 2 S) \pi \pi$ were studied. The authors found that the contribution from meson loops is comparable to those from the chiral contact terms and the $Z_{b^{-}}$ exchange terms. With the inclusion of $Z_{b}$ 's and meson loops, the authors can reproduce the experimental two-hump behavior of the $\pi \pi$ spectra in $\Upsilon(4 S) \rightarrow \Upsilon(2 S) \pi \pi$. In the process $\Upsilon(4 S) \rightarrow \Upsilon(1 S) \pi \pi$, they expect a narrow dip around $1 \mathrm{GeV}$ in the $\pi \pi$ invariant mass distribution.

In Ref. [824], Guo et al studied the line shapes of the near-threshold states $Z_{b}(10610)$ and $Z_{b}(10650)$ with $I^{G}\left(J^{P C}\right)=1^{+}\left(1^{+-}\right)$using their parametrization method. They found that the $Z_{b}(10610)$ is a virtual state on the second Riemann sheet near the $B \bar{B}^{*}$ threshold, while the $Z_{b}(10650)$ is a resonance on the third or fourth Riemann sheet near the $B^{*} \bar{B}^{*}$ threshold.

In Ref. [825], Bondar and Voloshin discussed the possibility that the production channels in the $\Upsilon(6 S)$ mass region are dominated by the $Z_{b}(10610)$ (not $Z_{b}(10650)$ ) due to the triangle singularity in $e^{+} e^{-} \rightarrow B_{1}(5721) \bar{B} \rightarrow Z_{b}(10610) \pi$. If this mechanism is dominant, any nonresonant background not associated with the $Z_{b}(10610)$ should be suppressed. A similar structure near $11.06 \mathrm{GeV}$ in the $e^{+} e^{-}$ annihilations into $\Upsilon(n s) \pi \pi$ and $h_{b}(k P) \pi \pi$ was also expected.

In Ref. [826], the hidden-bottom decays of the $Z_{b}(10610)$ and $Z_{b}(10650)$ into $\Upsilon(n S) \pi(n=1,2,3)$, $\eta_{b}(m S) \rho(m=1,2)$, and $\eta_{b}(m S) \gamma$ were analyzed with the effective Lagrangian approach, via final state interactions or meson loop contributions. With this mechanism, the final state interactions were found to be important, and the branching ratios into $\eta_{b}(m S) \rho$ and $\eta_{b}(m S) \gamma$ were predicted. In the same framework, the hidden-charm decays of the $Z_{c}(3900)$ and $Z_{c}(4020)$ states in Ref. [827] were consistent with the recent BESIII measurement [828].

In Ref. [829], the bottomoniumlike $Z_{b}(10610)$ and $Z_{b}(10650)$ resonances were proposed to be a mixture of the molecular $B \bar{B}^{*} / B^{*} \bar{B}$ and $B^{*} \bar{B}^{*}$ states with one mixing angle $\theta$. With $\theta \approx 0.2$, the ratio $\Gamma\left[Z_{b}(10650) \rightarrow B \bar{B}^{*} / B^{*} \bar{B}\right] / \Gamma\left[Z_{b}(10650) \rightarrow B^{*} \bar{B}^{*}\right] \sim 0.04$ and a definite interference pattern in the process $\Upsilon(5 S) \rightarrow B \bar{B}^{*} / B^{*} \bar{B} \pi$ were predicted.

In Ref. [830], the experimental data for the two $Z_{b}$ states were analyzed simultaneously in the molecule picture using the Lippmann-Schwinger equations. The long-range pion interaction does not affect the line shapes if only $S$-waves are considered, but the situation is slightly different once $D$-waves are included. The study also indicates that the two $Z_{b}$ states can be described by poles on the unphysical Riemann sheets, where the $Z_{b}(10610)$ is associated with a virtual state just below the $B \bar{B}^{*}$ threshold, while the $Z_{b}(10650)$ is likely an above-threshold shallow state. In Ref. [831], the same method was 
applied to the spin partner states $W_{b}$ 's with $J^{P C}=(0,1,2)^{++}$. The pionful (pionless) approach leads to the threshold cusp (above-threshold hump) in the line shapes.

In Ref. [832], the production of $Z_{b}(10610)$ and $Z_{b}(10650)$ from the $\Upsilon(5 S, 6 S)$ decays through bottommeson loops was investigated. The contributions from the $B_{1}^{\prime} \bar{B}^{(*)} / B_{0}^{*} \bar{B}^{*}$ loops dominate those from the $B^{(*)} \bar{B}^{(*)}$ loops due to the larger pionic couplings in the triangle diagrams. The branching ratios of $\Upsilon(6 S) \rightarrow Z_{b}(10610) \pi$ and $Z_{b}(10650) \pi$ were predicted to be around $4 \%$.

In Ref. [833], the strong decays of $Z_{b}(10610)$ and $Z_{b}(10650)$ were studied with molecular type currents using the same method in studying the decay widths of $Z_{c}(3900)$ and $Z(4430)$ [819]. The $\Upsilon(1 S) \pi$ and $\eta_{b} \rho$ decay modes are suppressed but not as much as the Belle data.

Inspired by BESIII's new measurement $\Gamma\left(Z_{c}(3900) \rightarrow \rho \eta_{c}\right) / \Gamma\left(Z_{c} \rightarrow \pi J / \psi\right)=2.1 \pm 0.8$ [828], Voloshin estimated the cross section of the processes $e^{+} e^{-} \rightarrow \gamma W_{b J}$ at the maximum of the $\Upsilon(5 S)$ resonance to be about $0.1 \mathrm{pb}$ [834], where $W_{b J}$ are the predicted isovector $B^{(*)} \bar{B}^{(*)}$ meson-antimeson states from the considerations of the heavy quark spin symmetry and light quark spin symmetry [406].

\section{$10.2 Q Q \bar{q} \bar{q}$ and $Q Q \bar{Q} \bar{Q}$}

In Ref. [835], the feasibility of the production and detection of $T_{c c}$ at various facilities was discussed. Its production is comparable to the doubly charmed baryon production and prompt $J / \psi c \bar{c}$ production. Thus, its observations at SELEX, LHC, and Belle should be possible.

The production of the $T_{c c}$ states in the electron-positron process has been discussed in Refs. [161, 162, 739]. To search for these exotic states at $B$ factories, the authors of Ref. [739] proposed a more efficient analysis method. If a three-jet event may be identified by their energy and angular distributions, the doubly charmed states can be searched for in the most energetic jet, which may suppress the combination background fluctuations of the reconstructed hadron mass spectra.

In [836], the authors argued that the doubly charmed tetraquarks are a straightforward consequence of the diquark-antidiquark model [95, 97]. Assuming that these tetraquarks are above-threshold particles, the authors explored their decay modes and rates as well as their production mechanisms. They showed that the doubly charmed tetraquarks could be produced at LHC from the decays of $B_{c}$ and $\Xi_{b c}$.

In Ref. [837], the authors noted that the hadronic effects on the $T_{c c}$ tetraquark state are insignificant in relativistic heavy ion collisions. The authors also noticed that the $D^{+} D^{+} \pi^{-}$and $D^{0} D^{0} \pi^{+}$decay modes of $T_{c c}$ should be the most probable channels to reconstruct it from heavy ion collisions. Besides, the order of yields at LHC and RHIC reads, $D>\Xi_{c c}^{(*)}>T_{c c}$ (molecule) $>T_{c c}$ (compact).

In Ref. [838], Ali et al explored the production of $T_{[\bar{u} \bar{d}]}^{\{b b\}}, T_{[\bar{u} \bar{s}]}^{\{b b\}}, T_{[\bar{d} \bar{s}]}^{\{b b\}}$, and $\Xi_{b b q}(q=u, d, s)$, in $Z$-boson decays. The lifetimes of these tetraquarks were estimated to be around 0.5 ps. Besides, their signature decay modes were studied. In Ref. [839], the discovery potential of these tetraquarks at LHC was discussed. According to the estimated cross section $\sigma\left(p p \rightarrow T^{\{b b\}}+X\right)$, the prospects of discovering these states are excellent.

In Ref. [168], the semileptonic 2-, 3-, and 4-body weak decays of $T_{Q Q \bar{q} \bar{q}}(Q=c, b, q=u, d, s)$ were systematically investigated. The nonleptonic decays and possible golden decay channels at $\mathrm{LHCb}$ and Belle II experiments were also discussed. The weak decays of the $I\left(J^{P}\right)=0\left(1^{+}\right) T_{b b ; \bar{u} \bar{d}}^{-}$tetraquark state to the scalar diquark-antidiquark $Z_{b c ; \bar{d} \bar{d}}=[b c][\bar{u} \bar{d}]$ were investigated in [520] using the QCD sum rule approach, and the lifetime of the $T_{b b ; \bar{u} \bar{d}}^{-}$was found to be around $9.2 \mathrm{fs}$, which is shorter than that obtained in Ref. [838].

In Refs. [840, 841], the inclusive decay mode $\Xi_{b b q} \rightarrow B_{c}^{(*)-}+X_{c, s, q}$ was proposed as a potential discovery channel for the doubly bottom baryon at LHC, which is based on the fact that $B_{c}^{(*)}$ can only arise from the weak decay of a hadron with two bottom quarks. If the $\Xi_{b b q}$ states could be observed, such a decay mode may also be used to search for the bound $T_{b b}$ states.

The possible observation of the $[c c][\bar{c} \bar{c}]$ and $[b c][\bar{b} \bar{c}]$ states with $J^{P C}=2^{++}$at LHC was discussed 
in Refs. $[177,178]$. The color connections of $Q Q \bar{Q} \bar{Q}$ states and the induced hadronization effects on $\Xi_{c c}$ and $T_{c c}$ in $e^{+} e^{-}$annihilation processes were studied in [842]. A study of $T_{4 c}=c c \bar{c} \bar{c}$ production in double parton scattering was given in Ref. [756]. Possible channels to search for such a $b b \bar{b} \bar{b}$ state were discussed in Refs. [843, 844].

In Ref. [845], the authors investigated the lifetime and weak decays of the fully heavy tetraquark state $b b \bar{c} \bar{c}$ with $J^{P}=0^{+}$. They obtained a lifetime value around $0.1 \sim 0.3 \mathrm{ps}$ and discussed the golden channels to search for this state.

\section{$10.3 Q \bar{Q} q q q$}

The analysis of the process $\Lambda_{b} \rightarrow J / \psi K^{-} p$ in Ref. [846] by Roca, Nieves, and Oset supports the $P_{c}(4450)$ as a $3 / 2^{-}$molecule of mostly $\bar{D}^{*} \Sigma_{c}$ and $\bar{D}^{*} \Sigma_{c}^{*}$. In Ref. [847], an improved analysis by Roca and Oset indicates that the existence of the $P_{c}(4380)$ state cannot be undoubtedly claimed with only the fit to the $K^{-} p$ and $J / \psi p$ mass distributions. The investigation of $\Lambda_{b}^{0} \rightarrow J / \psi K^{-} p$ and $J / \psi p \pi^{-}$in Ref. [848] indicates that the s-/u-channel contributions are not negligible. The obtained bound states and line shape of the $J / \psi N$ mass distribution favor the assignment of the $P_{c}(4450)$ as a $\bar{D}^{*} \Sigma_{c}$ molecule.

In Ref. [849], the process $\Lambda_{b} \rightarrow J / \psi K^{0} \Lambda$ was studied with the contributions of a hidden-charm pentaquark-like molecule state $[184,193]$. A clear peak in the $J / \psi \Lambda$ mass distribution was found. In Ref. [850], the contributions of the hidden-charm $N^{*}$ states with $J^{P}=1 / 2^{-}$and $3 / 2^{-}$to the reaction $\gamma p \rightarrow \bar{D}^{* 0} \Lambda_{c}^{+}$were discussed. These states lead to clear peak structures in the total cross sections. The contributions of a hidden-beauty $N^{*}(11052)$ to the $\pi^{-} p$ scattering were discussed similarly in Ref. [851]. The authors of Ref. [852] suggested that a clean peak should be seen for a hidden-charm resonance (mass $4265 \mathrm{MeV}$ ), which couples to the $\eta_{c} p$ channel in the decay $\Lambda_{b} \rightarrow \eta_{c} K^{-} p$.

In Ref. [853], the production of the $P_{c}$ states and their yields in ultrarelativistic heavy ion collisions were discussed in both the compact pentaquark picture and the molecule picture. In Ref. [854], the production cross section for a hidden-charm pentaquark in proton-nucleus collisions was found to be sizable by assuming if it is a molecule of a charmonium and a light baryon.

In Ref. [855], Liu and Oka discussed the rescattering effects in the reaction $\pi^{-} p \rightarrow \pi^{-} J / \psi p$ due to the intermediate open-charm hadrons. They showed that the triangle singularity peaks can simulate the pentaquark-like resonances in the $J / \psi p$ invariant mass distributions. In Ref. [856], Guo et al proposed that the $P_{c}(4450)$ observed in the process $\Lambda_{b} \rightarrow J / \psi p K$ might be due to a triangle singularity around the $\chi_{c 1} p$ threshold. A discussion on triangle singularities in Ref. [857] indicates that the narrow $P_{c}(4450)$ would have an origin other than the triangle singularity from the $\Lambda^{*}$-charmonium-proton intermediate states, if this baryon has quantum numbers $J^{P}=3 / 2^{-}, 5 / 2^{+}$.

In studying the nature of $Z_{c}(3900)$ with the pole counting rule in Ref. [801], the authors suggested that the $P_{c}(4450)$ pentaquark can be related to $Z_{c}(3900)$ by replacing a $u d$ pair by a $\bar{d}$. They speculated that the $P_{c}(4450)$ should be a $\Sigma_{c} \bar{D}^{*}$ molecule with $J=3 / 2$ rather than $J=5 / 2$.

In Ref. [858], Kim et al considered the reaction $\pi^{-} p \rightarrow J / \psi n$ based on the hybridized Regge model and included the contributions of the $\mathrm{LHCb} P_{c}$ states. The total cross sections were found to be about $1 \mathrm{nb}$ in the vicinity of the heavy pentaquark masses. In Ref. [859], Karliner and Rosner discussed the $J / \psi N$ photoproduction on deuterium, through which the simultaneous investigation of the $c \bar{c} u u d$ and $c \bar{c} d d u$ isospin partner states can be achieved. The photoproduction of $P_{c}(4450)$ on a deuteron target may be used to check whether it is a genuine isospin-half resonance.

The authors of Ref. [860] studied the pentaquark production in the semileptonic decays $\Lambda_{b} \rightarrow$ $J / \psi p \ell^{-} \bar{\nu}_{\ell}$ where $\ell=e, \mu$. The branching ratios are about two orders of magnitude smaller than the decay $\Lambda_{b} \rightarrow p \mu \bar{\nu}_{\mu}$, which is accessible to the ongoing experiments at the LHCb.

In Ref. [740], the hidden-charm pentaquark production at the $e^{+} e^{-}$colliders was discussed in the NRQCD factorization framework, by treating the pentaquark as a $c \bar{c} n n n$ state with a color-octet $c \bar{c}$ configuration. It is possible to search for the direct pentaquark production signal at $e^{+} e^{-}$colliders. 
The authors of Ref. [219] discussed the production mechanism of the $P_{c}$ pentaquarks in $\Lambda_{b}$ decays and assumed that they are $(c \bar{c})_{8_{c}}(q q q)_{8_{c}}$ states. The decay happens through $b \rightarrow c+s+\bar{c}$ followed by a gluon emission $(\rightarrow u \bar{u})$ from $s$. Then $s$ and $\bar{u}$ form the kaon meson while the other quarks form the pentaquarks. The two color octet $(c \bar{c})_{8_{c}}$ and $(q q q)_{8_{c}}$ components in $S$-wave $(P$-wave) lead to the $J^{P}=3 / 2^{-}\left(5 / 2^{+}\right)$state. This mechanism can explain why only a few states with nonminimal constituents were discovered so far.

In Ref. [861], the difference between partial-wave analysis formalisms used in the construction of three-body amplitudes involving fermions was discussed, particularly in the decay $\Lambda_{b} \rightarrow J / \psi K^{-} p$. The authors found meaningful effects on the resonance pole position extraction, which is particularly relevant when several resonances overlap and the quantum number assignment is not stable. In Ref. [862], the authors discussed the role of the hidden-charm pentaquark resonance $P_{c}(4450)$ in $J / \psi$ photoproduction off ${ }^{12} \mathrm{C}$ and ${ }^{208} \mathrm{~Pb}$ target nuclei near threshold. The presence of $P_{c}(4450)$ produces additional enhancements above threshold in the total $J / \psi$ creation cross section on nuclei, which can be tested in future JLab experiments.

In Ref. [863], Voloshin discussed the hidden-charm pentaquark production in $\bar{p} d$ collisions, which is possible due to the coupling of charmonia to the $p \bar{p}$ channel. He found that the pentaquark formation may happen with the nucleons moving slowly inside the deuteron due to the masses of the pentaquark, charmonium, and the nucleon being close to a special kinetic relation. The formation cross section of hypothetical pentaquark states decaying to $\eta_{c} N$ was much larger than that of pentaquarks into $J / \psi N$, because of a much larger $\eta_{c} \rightarrow p \bar{p}$ decay width.

The authors of Ref. [864] studied the strong decay mode $J / \psi p$ of the $P_{c}$ states in the molecule scenario. The partial decay widths are significantly different for various $J^{P}$ assignments. The $S$-wave $\Sigma_{c} \bar{D}^{*}$ pictures for the $P_{c}(4380)$ and $P_{c}(4450)$ and the $\Sigma_{c}^{*} \bar{D}$ assignment for the $P_{c}(4380)$ with $J^{P}=3 / 2^{-}$ are all allowed by the present experimental data.

In Ref. [865], the different decay properties of the $\bar{D} \Sigma_{c}^{*}$ and $\bar{D}^{*} \Sigma_{c}$ molecules were discussed. The $P_{c}(4380)$ was proposed as a $\bar{D} \Sigma_{c}^{*}$ molecule and the decay channel $\bar{D}^{*} \Lambda_{c}$ was suggested to be used to disentangle its nature. In Ref. [866], the decay properties of the $P_{c}(4380)$ and $P_{c}(4450)$ were studied with the effective Lagrangian framework by treating them as meson-baryon molecules. The $\bar{D} \Sigma_{c}^{*}$ and $\bar{D}^{*} \Sigma_{c}$ molecules have different decay branching ratios. The $P_{c}(4380)\left(P_{c}(4450)\right)$ was proposed to be a $3 / 2^{-} \bar{D} \Sigma_{c}^{*}\left(5 / 2^{+} \bar{D}^{*} \Sigma_{c}\right)$ molecule. The cross sections for the processes $\gamma p \rightarrow J / \psi p$ and $\pi p \rightarrow J / \psi p$ through the $S$-channel $P_{c}$ states were also calculated. The same framework was used to discuss the decay behaviors of the strange and beauty partners of the $P_{c}$ hadronic molecules in Ref. [867].

Besides the spectrum, the authors of Ref. [375] also calculated the partial decay width of the $J^{P}=3 / 2^{-} P_{c}(4450)$ into $J / \psi N$ to be around $11 \mathrm{MeV}$ by treating it as a hadroquarkonium state. Later in Ref. [868], the decays of the pentaquark $P_{c}(4450)$ were further investigated in both hadrocharmonium and molecular pictures. The authors found that the decay patterns are vastly different. The decays of the $P_{c}$ molecule into $J / \psi$ are strongly suppressed, while the opposite happens in the hadrochamonium case.

In Ref. [524], the authors studied the strong decay width of $P_{c}(4380)$ into $J / \psi N$ by treating it as a $\bar{D}^{*} \Sigma_{c}$ molecule with $J^{P}=3 / 2^{+}$or $3 / 2^{-}$. The coupling constants were calculated with QCD sum rules, and the obtained decay width is around $187(213) \mathrm{MeV}$ for the case $P=+(P=-)$.

After the new pentaquark states $P_{c}(4312), P_{c}(4440)$, and $P_{c}(4457)$ were announced [68], Guo, Jing, Meissner and Sakai suggested to search for the $P_{c}(4457)$ in the $J / \psi \Delta$ mode in order to test its nature [382]. If the state is a $\Sigma_{c} \bar{D}^{*}$ molecule, the authors pointed out that the isospin breaking decay ratio $\mathcal{B}\left(P_{c}(4457) \rightarrow J / \psi \Delta^{+}\right) / \mathcal{B}\left(P_{c}(4457) \rightarrow J / \psi p\right)$ would be at the level ranging from a few percent to about $30 \%$. 


\subsection{A short summary}

We give a short summary for the production and decay properties of the heavy tetraquark and pentaquark states based on the recent studies in the literature.

- The $X$ states. In the conventional charmonium picture, the $X(3940)$ could be the $\eta_{c}(3 S)$ while the $X(4140)$ or $X(4274)$ could be the $\chi_{c 1}(3 P)$. In the molecule picture, the $X(4160)$ could be a $D_{s}^{*} \bar{D}_{s}^{*}$ molecule.

- The $Y$ states. From the theoretical studies of production and decay, it seems that not all $Y$ states (Y(4008), $Y(4220) / Y(4230), Y(4260), Y(4320), Y(4360), Y(4390), Y(4630)$, and $Y(4660))$ shall exist. The interference effects, coupled channel effects, or final state interactions may make several genuine states behave like more resonance signals. Up to now, we have much deeper understandings about the nature of $X(3872)$ than 15 years ago, although more experimental measurements are still needed. Different from the $X(3872)$, the $J^{P C}=1^{--}$structures in the mass region $4.0 \sim 4.6 \mathrm{GeV}$ seem more complicated and challenging. The conventional charmonium states, theoretically expected but elusive hybrid charmonium mesons, and molecular states coincide here. Do they mix? The nature of $Y(4260)$ is still far from being understood, although it was observed almost 15 years ago.

- The $Z_{c}$ and $Z_{b}$ states. The studies of productions and decays favor the molecule assignments for the $Z_{c}(3900)$ and $Z_{c}(4020)$. Whether the $Z_{c}(4430)$ state is a molecule, a compact tetraquark, or a structure induced by triangle singularity is still unclear. One cannot distinguish the nature of $Z_{c}(4100)$ with the present experimental $B$ decay data, either. Is it a $\mathrm{P}$-wave $D^{*} \bar{D}^{*}$ resonance or a state caused by final state interaction effects? The $Z_{b}(10610)$ and $Z_{b}(10650)$ should be the $B \bar{B}^{*}$ and $B^{*} \bar{B}^{*}$ molecular states, respectively.

- The $Q \bar{Q} q q q$ states. The $\Sigma_{c}^{*} \bar{D}$ and $\Sigma_{c} \bar{D}^{*}$ molecule assignments for $P_{c}(4380)$ and $P_{c}(4450)$, respectively, can explain experimental measurements for their production and decay properties, although their nonresonance interpretations cannot be excluded.

- So far, a large number of investigations on exotic productions have been presented in the literature while the investigations on decays are still lacking. Further studies on decay properties of the exotic hadrons, especially at the quark level, are necessary, which play a key role in understanding the inner structures of the exotic states. On the experimental side, searching for expected multiquarks, checking predicted features, and measuring more physical quantities are definitely needed.

- At present, all the multiquark candidates are produced at $e^{+} e^{-}, e^{+} p, p p, p A$, and $A A$ colliders. Future $p \bar{p}, \bar{p} A$, and $e A$ colliders are still under construction. Besides these collision processes, the $\gamma \gamma, \gamma A$ interactions, charmonia decays, bottomonia decays, $B$ decays, and $\Lambda_{b}$ decays are also used to study the multiquark states. From the experimental fact that all the confirmed exotic candidates were observed in the $e^{+} e^{-}\left(\gamma^{*}\right), \gamma \gamma$, or hadron decay processes, it seems that producing multiquark states in multiproduction processes is very difficult and undetectable [869, 741]. If this is true, searching for the conventional $b b b$ baryons should be more feasible and important than searching for $b b \bar{b} \bar{b}$ compact states. The exotic hadrons we may observe in the near future would be dominantly states in the charm sector. They are indeed the structures being studied at LHC (see Table 26 of Ref. [870]).

\section{Summary and perspective}

At the end of this review, we would give a brief summary and perspective for the progress on the multiquark physics. It is useful and instructive to recall the establishment of the quark model before 
we discuss the multiquark systems. The quark model was first proposed in 1964 to deal with the classification of hadrons. In 1974, the discovery of the $J / \psi$ meson gave the direct evidence for the existence of the charm quark, which was known as "November Revolution". Actually, the existence of the charm quark was already predicted in the GIM mechanism before its observation [11]. The bottom quark was discovered by E288 experiment in 1977 at Fermilab. After that, plenty of bound states and excitations of the heavy mesons and baryons have been observed in the following decades [35]. We show the roadmap of the conventional hadrons in Fig. 30.

Although we still can't understand QCD confinement from the first principle, the quark model has achieved indubitable success in systematizing the properties of mesons and baryons. For the exotic hadron states such as the multiquark systems, one may turn to those phenomenological models for help which are closely related to QCD since the rigorous solutions of soft QCD are still unavailable. The interplay between the phenomenological models and experimental observations will also improve their prediction capabilities. The discovery of the $\Xi_{c c}(3621)$ in 2017 was a successful example for the collaboration between experimenters and theorists. Inspired by this observation, one will further expect the future discovery of the $\Omega_{c c c}$ baryon.

\section{Roadmap of conventional hadrons}

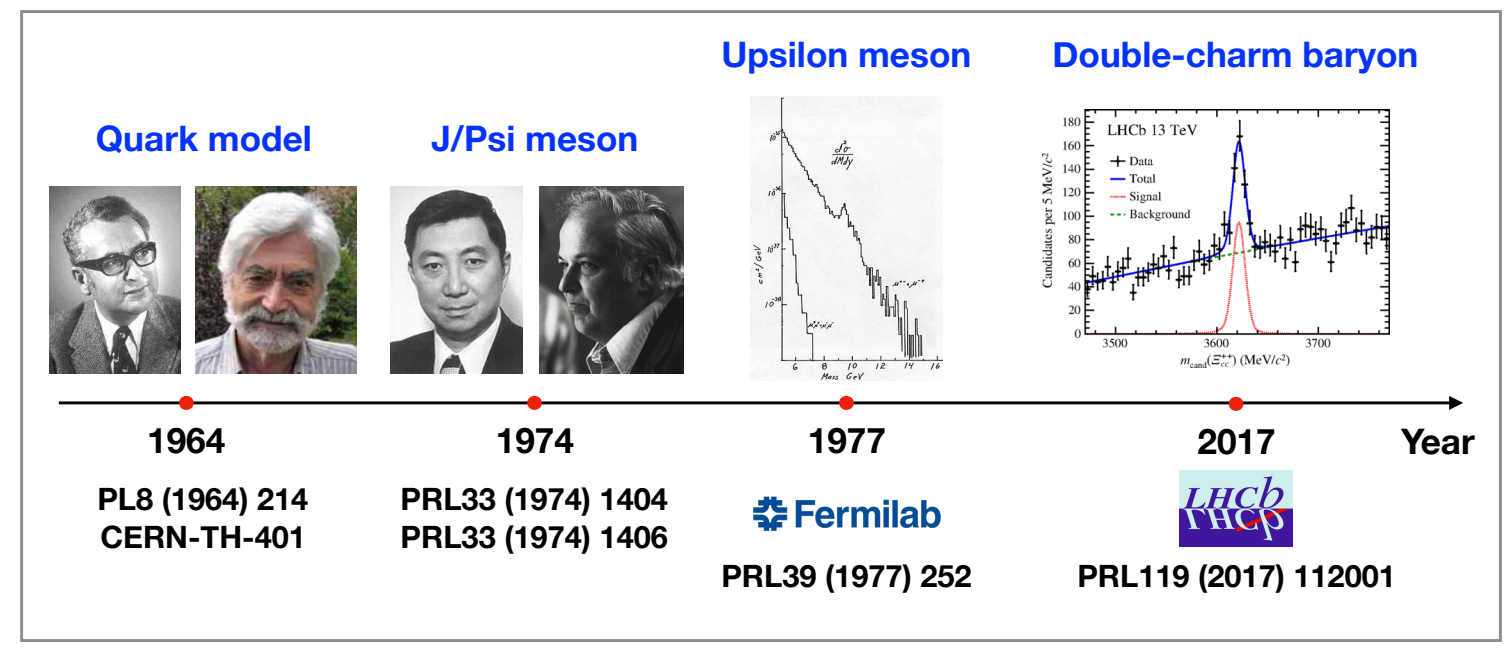

Figure 30: The roadmap of the conventional hadrons.

Searching for the multiquark states is another central issue of the hadron physics. In the past several decades, there accumulated abundant investigations on tetraquarks and pentaquarks based on various phenomenological models. However, the progress was still rudimentary and far away from our expectation due to the lack of the sufficiently accurate experimental results. The year of 2003 is very important in the history of the exotic hadrons. Since 2003, lots of quarkonium-like $X Y Z$ states and pentaquark $P_{c}$ states have been observed in the modern facilities, such as BaBar, Belle, CLEO-c, D0, CDF, BESIII, and LHCb etc.

The desires for the nature of these new hadron states have promoted the model building. Some model predictions agree with the experimental data. For example, the masses of some $X Y Z$ states can be reproduced in the tetraquark configurations. However, there are still many observables which can not be computed accurately enough to be compared with the experimental measurements, e. g., the branching fractions and decay widths for some important channels. Moreover, some new resonances can usually be explained in different models, implying the present phenomenological investigations are unable to distinguish their underlying structures. More efforts are needed to bring down the error bars in future.

Nevertheless, we can still find some critical breakthroughs in the phenomenological aspect. The 
investigations for the doubly heavy tetraquarks with various methods such as the QCD sum rules [515], CMI model [109, 166], LQCD [720], and the heavy-quark symmetry prediction [164] lead to consistent predictions that the doubly bottom $u d \bar{b} \bar{b}$ tetraquark states would probably be stable against the strong interaction. Such an agreement may imply critical interaction mechanisms in these tetraquark systems, which can be a breaking point for our understanding of the multiquark states. To date, most of the multiquark candidates were observed in the charmonium sector, such as the hidden-charm XYZ states and $P_{c}$ pentaquark states. From the theoretical point of view, more tetraquark and pentaquark states with different flavor and color structures and different quantum numbers should also be possible. In the past several years, the authors have made some efforts along this direction. We predicted the pentaquark states with hidden-charm and open-strange flavors. Based on the discovery of $\Xi_{c c}(3621)$, we studied the interactions between the $\Xi_{c c}(3621)$ baryon and $S$-wave charmed mesons and predicted the existence of the triply charmed pentaquark states.

Last but not the least, we shall emphasize the unique and pivotal role of the lattice QCD in the search of the multiquark states. The lattice QCD simulations start from the first principle and can investigate the few-hadron reaction processes and extract important information such as the resonance properties, the hadronic interactions, scattering amplitudes, phase shifts, binding energies, mass spectra, decay widths and so on. In the past few years, tremendous lattice results from quenched to unquenched calculations become accurate enough to be compared with both the experimental data and phenomenological results. Especially the simulations using a large basis of operators including the multi-hadron operators have become a unique, vital and powerful tool to explore the near-threshold exotic states. Some very successful examples include the lattice QCD investigations of the $X(3872)$ and $Z_{c}(3900)$ states, the doubly bottom tetraquark states, which provide very clear and intuitive picture of the underlying structures of the exotic states. However, only the $\eta_{c}-N$ and $J / \psi-N$ scattering processes were studied in lattice QCD for the pentaquark systems, which is far away from pinning down the existence of the hidden-charm pentaquark states. In terms of LHCb's recent discovery of the three hidden-charm pentaquark states, the lattice QCD simulations of the charmed baryon and anti-charmed meson are badly needed. At the present stage, many lattice simulations were performed at the unphysically large pion mass due to the expensive computational cost. However, the existence of the molecular type of exotic hadrons is very sensitive to the pion mass since the pion exchange force decays exponentially with the pion mass. Further dynamical lattice QCD simulations using ensembles with near-physical or even the physical pion mass will be extremely valuable for the $P_{c}$ systems. As shown in Fig. 31, the interplay among experiments, phenomenological models and lattice QCD and their joint progress will definitely sharpen our understanding of the new hadron spectroscopy and QCD itself.

When we were preparing this review, the LHCb Collaboration reported a new charmonium state with $J^{P C}=3^{--}$via $p p$ collision in the PHI-PSI 2019 workshop. The BESIII Collaboration has observed the processes $e^{+} e^{-} \rightarrow \pi^{+} \pi^{-} \psi(3770)$ and $D_{1}(2420)^{0} \bar{D}^{0}+$ c.c. for the first time [871] and measured the cross section of the process $e^{+} e^{-} \rightarrow \omega \chi_{c 0}$ [872], which provided new useful information to study the $X Y Z$ states. Very recently, LHCb has observed CP violation in the charmed meson decays [873], which is definitely an important milestone in the history of particle physics. These excellent observations at LHCb demonstrated its distinguished performances and capabilities to study the exotic hadron states. The Belle II Collaboration released the Belle II Physics Book [874] in 2018 and started to take data with a fully instrumented detector in March, 2019. Although the main aim of Belle II experiment is searching for CP-violation and revealing violations of the symmetry between particles and anti-particles, it will also search for the exotic hadrons and make precision measurements of their properties. To some extent, BelleII shall become a factory of the charmonium-like $X Y Z$ states. The BESIII experiment at BEPCII will continue to contribute to the field of the $X Y Z$ states in the near future. They will perform unique investigations on the $X Y Z$ states around $\sqrt{s}=4.2 \mathrm{GeV}, 4.38 \mathrm{GeV}$ and $4.6 \mathrm{GeV}$. Moreover, the upcoming PANDA experiment will also study the multiquark and other exotic hadrons in future. The coming decade shall witness a new landscape of the hadron spectroscopy. 


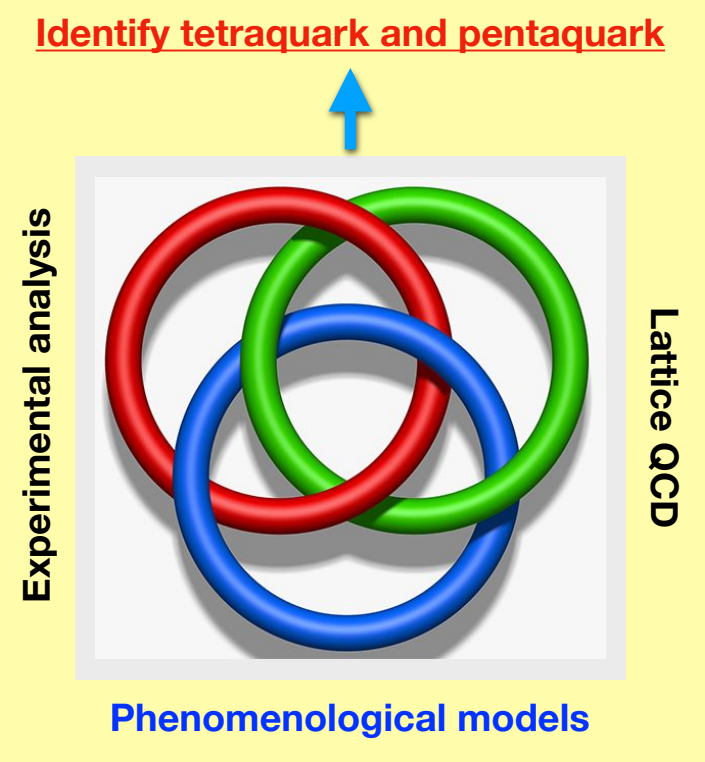

Figure 31: The interplay of the experimental analyses, phenomenological models and lattice QCD on the understanding of the new hadron spectroscopy.

\section{Acknowledgments}

We would like to express our gratitude to all the collaborators and colleagues who contributed to the investigations presented here, in particular to Dian-Yong Chen, Rui Chen, Xiao-Lin Chen, Er-Liang Cui, Wei-Zhen Deng, Jun He, Ning Li, Shi-Yuan Li, Xiao-Hai Liu, Yu-Nan Liu, Zhi-Gang Luo, Li Ma, Takayuki Matsuki, Zong-Guo Si, T. G. Steele, Zhi-Feng Sun, Guan-Juan Wang, Jing Wu, Lu Zhao, Zhi-Yong Zhou. We thank Er-Liang Cui for helping prepare some relevant documents. This project is supported by the National Natural Science Foundation of China under Grants No. 11722540, No. 11775132, No. 11575008, No. 11621131001 and National Key Basic Research Program of China (2015CB856700), the China National Funds for Distinguished Young Scientists under Grant No. 11825503, the Fundamental Research Funds for the Central Universities, and the National Program for Support of Top-notch Young Professionals.

\section{References}

[1] H.-X. Chen, W. Chen, X. Liu, S.-L. Zhu, The hidden-charm pentaquark and tetraquark states, Phys. Rept. 639 (2016) 1-121. arXiv:1601.02092, doi:10.1016/j.physrep.2016.05.004.

[2] L. B. Okun, The theory of weak interaction, in: High-energy physics. Proceedings, 11th International Conference, ICHEP'62, Geneva, Switzerland, Jul 4-11, 1962, 1962, pp. 845-866.

[3] M. Gell-Mann, A Schematic Model of Baryons and Mesons, Phys. Lett. 8 (1964) 214-215. doi: 10.1016/S0031-9163(64)92001-3.

[4] G. Zweig, An SU(3) model for strong interaction symmetry and its breaking. Version 2, in: D. Lichtenberg, S. P. Rosen (Eds.), DEVELOPMENTS IN THE QUARK THEORY OF HADRONS. VOL. 1. 1964 - 1978, 1964, pp. 22-101.

[5] G. Zweig, An SU(3) model for strong interaction symmetry and its breaking. Version 1, 1964. 
[6] V. E. Barnes, et al., Observation of a Hyperon with Strangeness -3, Phys. Rev. Lett. 12 (1964) 204-206. doi:10.1103/PhysRevLett.12.204.

[7] M. Gell-Mann, The Eightfold Way: A Theory of strong interaction symmetry, 1961.

[8] Y. Ne'eman, Derivation of strong interactions from a gauge invariance, Nucl. Phys. 26 (1961) 222-229. doi:10.1016/0029-5582(61)90134-1.

[9] J. J. Aubert, et al., Experimental Observation of a Heavy Particle J, Phys. Rev. Lett. 33 (1974) 1404-1406. doi:10.1103/PhysRevLett.33.1404.

[10] J. E. Augustin, et al., Discovery of a Narrow Resonance in $e^{+} e^{-}$Annihilation, Phys. Rev. Lett. 33 (1974) 1406-1408, [Adv. Exp. Phys. 5, 141 (1976)]. doi:10.1103/PhysRevLett.33.1406.

[11] S. L. Glashow, J. Iliopoulos, L. Maiani, Weak Interactions with Lepton-Hadron Symmetry, Phys. Rev. D2 (1970) 1285-1292. doi:10.1103/PhysRevD.2.1285.

[12] G. S. Abrams, et al., The Discovery of a Second Narrow Resonance in e+ e- Annihilation, Phys. Rev. Lett. 33 (1974) 1453-1455, [Adv. Exp. Phys.5,150(1976)]. doi:10.1103/PhysRevLett. 33. 1453.

[13] P. A. Rapidis, et al., Observation of a Resonance in $e^{+} e^{-}$Annihilation Just Above Charm Threshold, Phys. Rev. Lett. 39 (1977) 526, [Erratum: Phys. Rev. Lett.39,974(1977)]. doi: 10.1103/PhysRevLett.39.526,10.1103/PhysRevLett.39.974.

[14] G. Goldhaber, et al., $D$ and $D^{*}$ Meson Production Near 4-GeV in $e^{+} e^{-}$Annihilation, Phys. Lett. 69B (1977) 503-507. doi:10.1016/0370-2693(77)90855-3.

[15] R. Brandelik, et al., Total Cross-section for Hadron Production by $e^{+} e^{-}$Annihilation at Centerof-mass Energies Between 3.6 GeV and 5.2 GeV, Phys. Lett. B76 (1978) 361. doi:10.1016/ 0370-2693 (78) 90807-9.

[16] J. Siegrist, et al., Observation of a Resonance at 4.4-GeV and Additional Structure Near 4.1-GeV in $e^{+} e^{-}$Annihilation, Phys. Rev. Lett. 36 (1976) 700. doi:10.1103/PhysRevLett.36.700.

[17] E. Eichten, K. Gottfried, T. Kinoshita, J. B. Kogut, K. D. Lane, T.-M. Yan, The Spectrum of Charmonium, Phys. Rev. Lett. 34 (1975) 369-372, [Erratum: Phys. Rev. Lett. 36, 1276 (1976)]. doi:10.1103/PhysRevLett.34.369.

[18] E. Eichten, K. Gottfried, T. Kinoshita, K. D. Lane, T.-M. Yan, Charmonium: The Model, Phys. Rev. D17 (1978) 3090, [Erratum: Phys. Rev. D21, 313 (1980)]. doi:10.1103/PhysRevD.17. 3090, 10.1103/PhysRevD.21.313.

[19] E. Eichten, K. Gottfried, T. Kinoshita, K. D. Lane, T.-M. Yan, Charmonium: Comparison with Experiment, Phys. Rev. D21 (1980) 203. doi:10.1103/PhysRevD.21.203.

[20] R. Barbieri, R. Kogerler, Z. Kunszt, R. Gatto, Meson Masses and Widths in a Gauge Theory with Linear Binding Potential, Nucl. Phys. B105 (1976) 125-138. doi :10.1016/0550-3213(76) 90064-X.

[21] D. P. Stanley, D. Robson, Nonperturbative Potential Model for Light and Heavy Quark anti-Quark Systems, Phys. Rev. D21 (1980) 3180-3196. doi:10.1103/PhysRevD.21.3180.

[22] J. Carlson, J. B. Kogut, V. R. Pandharipande, Hadron Spectroscopy in a Flux Tube Quark Model, Phys. Rev. D28 (1983) 2807. doi:10.1103/PhysRevD.28.2807. 
[23] J. L. Richardson, The Heavy Quark Potential and the Upsilon, J/psi Systems, Phys. Lett. 82B (1979) 272-274. doi:10.1016/0370-2693(79)90753-6.

[24] W. Buchmuller, G. Grunberg, S. H. H. Tye, The Regge Slope and the Lambda Parameter in QCD: An Empirical Approach via Quarkonia, Phys. Rev. Lett. 45 (1980) 103, [Erratum: Phys. Rev. Lett.45,587(1980)]. doi:10.1103/PhysRevLett.45.103,10.1103/PhysRevLett.45.587.

[25] W. Buchmuller, S. H. H. Tye, Quarkonia and Quantum Chromodynamics, Phys. Rev. D24 (1981) 132. doi:10.1103/PhysRevD.24.132.

[26] A. Martin, A Simultaneous FIT of B anti-B, C anti-C, S anti-S, (BCS Pairs) and C anti-S Spectra, Phys. Lett. 100B (1981) 511-514. doi:10.1016/0370-2693(81)90617-1.

[27] G. Bhanot, S. Rudaz, A New Potential for Quarkonium, Phys. Lett. 78B (1978) 119-124. doi: 10.1016/0370-2693(78)90362-3.

[28] C. Quigg, J. L. Rosner, Quarkonium Level Spacings, Phys. Lett. 71B (1977) 153-157. doi: 10.1016/0370-2693(77)90765-1.

[29] L. P. Fulcher, Perturbative QCD, a universal QCD scale, long range spin orbit potential, and the properties of heavy quarkonia, Phys. Rev. D44 (1991) 2079-2084. doi:10.1103/PhysRevD.44. 2079.

[30] S. N. Gupta, J. M. Johnson, W. W. Repko, C. J. Suchyta, III, Heavy quarkonium potential model and the p wave singlet state of charmonium, Phys. Rev. D49 (1994) 1551-1555. arXiv: hep-ph/9312205, doi:10.1103/PhysRevD.49.1551.

[31] J. Zeng, J. W. Van Orden, W. Roberts, Heavy mesons in a relativistic model, Phys. Rev. D52 (1995) 5229-5241. arXiv:hep-ph/9412269, doi:10.1103/PhysRevD.52.5229.

[32] D. Ebert, R. N. Faustov, V. O. Galkin, Properties of heavy quarkonia and $B_{c}$ mesons in the relativistic quark model, Phys. Rev. D67 (2003) 014027. arXiv:hep-ph/0210381, doi:10.1103/ PhysRevD.67.014027.

[33] S. Godfrey, N. Isgur, Mesons in a Relativized Quark Model with Chromodynamics, Phys. Rev. D32 (1985) 189-231. doi:10.1103/PhysRevD.32.189.

[34] S. Capstick, N. Isgur, Baryons in a Relativized Quark Model with Chromodynamics, Phys. Rev. D34 (1986) 2809, [AIP Conf. Proc.132,267(1985)]. doi:10.1103/PhysRevD.34.2809, 10.1063/ 1.35361.

[35] M. Tanabashi, et al., Review of Particle Physics, Phys. Rev. D98 (3) (2018) 030001. doi:10. 1103/PhysRevD.98.030001.

[36] R. Aaij, et al., Observation of the doubly charmed baryon $\Xi_{c c}^{++}$, Phys. Rev. Lett. 119 (11) (2017) 112001. arXiv:1707.01621, doi:10.1103/PhysRevLett.119.112001.

[37] E. Klempt, J.-M. Richard, Baryon spectroscopy, Rev. Mod. Phys. 82 (2010) 1095-1153. arXiv: 0901.2055, doi:10.1103/RevModPhys.82.1095.

[38] E. Klempt, A. Zaitsev, Glueballs, Hybrids, Multiquarks. Experimental facts versus QCD inspired concepts, Phys. Rept. 454 (2007) 1-202. arXiv:0708.4016, doi:10.1016/j.physrep.2007.07. 006. 
[39] J. Z. Bai, et al., Observation of a near threshold enhancement in th $p \bar{p}$ mass spectrum from radiative $J / \psi \rightarrow \gamma p \bar{p}$ decays, Phys. Rev. Lett. 91 (2003) 022001. arXiv:hep-ex/0303006, doi: 10.1103/PhysRevLett.91.022001.

[40] M. Ablikim, et al., Observation of a resonance X(1835) in $J / \psi \rightarrow \gamma \pi^{+} \pi^{-} \eta^{\prime}$, Phys. Rev. Lett. 95 (2005) 262001. arXiv:hep-ex/0508025, doi:10.1103/PhysRevLett.95.262001.

[41] M. Ablikim, et al., Confirmation of the $X(1835)$ and observation of the resonances $X(2120)$ and $X(2370)$ in $J / \psi \rightarrow \gamma \pi^{+} \pi^{-} \eta^{\prime}$, Phys. Rev. Lett. 106 (2011) 072002. arXiv:1012.3510, doi: 10.1103/PhysRevLett.106.072002.

[42] M. Ablikim, et al., Observation of a near-threshold enhancement in the omega phi mass spectrum from the doubly OZI suppressed decay J / psi -i gamma omega phi, Phys. Rev. Lett. 96 (2006) 162002. arXiv:hep-ex/0602031, doi:10.1103/PhysRevLett.96.162002.

[43] M. Ablikim, et al., Observation of $\mathrm{Y}(2175)$ in J / psi — i eta phi f(0)(980), Phys. Rev. Lett. 100 (2008) 102003. arXiv:0712.1143, doi:10.1103/PhysRevLett.100.102003.

[44] M. Ablikim, et al., Study of $J / \psi \rightarrow \eta \phi \pi^{+} \pi^{-}$at BESIII, Phys. Rev. D91 (5) (2015) 052017. arXiv:1412.5258, doi:10.1103/PhysRevD.91.052017.

[45] D. Diakonov, V. Petrov, M. V. Polyakov, Exotic anti-decuplet of baryons: Prediction from chiral solitons, Z. Phys. A359 (1997) 305-314. arXiv:hep-ph/9703373, doi:10.1007/s002180050406.

[46] T. Nakano, et al., Evidence for a narrow $S=+1$ baryon resonance in photoproduction from the neutron, Phys. Rev. Lett. 91 (2003) 012002. arXiv:hep-ex/0301020, doi:10.1103/ PhysRevLett.91.012002.

[47] S.-L. Zhu, Pentaquarks, Int. J. Mod. Phys. A19 (2004) 3439-3469. arXiv:hep-ph/0406204, doi:10.1142/S0217751X04019676.

[48] T. Liu, Y. Mao, B.-Q. Ma, Present status on experimental search for pentaquarks, Int. J. Mod. Phys. A29 (13) (2014) 1430020. arXiv:1403.4455, doi:10.1142/S0217751X14300208.

[49] A. Martinez Torres, E. Oset, A novel interpretation of the ' $\Theta^{+}(1540)$ pentaquark' peak, Phys. Rev. Lett. 105 (2010) 092001. arXiv:1008.4978, doi:10.1103/PhysRevLett.105.092001.

[50] B. Aubert, et al., Observation of a narrow meson decaying to $D_{s}^{+} \pi^{0}$ at a mass of $2.32 \mathrm{GeV} / \mathrm{c}^{2}$, Phys. Rev. Lett. 90 (2003) 242001. arXiv:hep-ex/0304021, doi:10.1103/PhysRevLett.90.242001.

[51] T. Barnes, F. E. Close, H. J. Lipkin, Implications of a $D K$ molecule at 2.32 GeV, Phys. Rev. D68 (2003) 054006. arXiv:hep-ph/0305025, doi:10.1103/PhysRevD.68.054006.

[52] H.-Y. Cheng, W.-S. Hou, B decays as spectroscope for charmed four quark states, Phys. Lett. B566 (2003) 193-200. arXiv:hep-ph/0305038, doi:10.1016/S0370-2693(03)00834-7.

[53] A. P. Szczepaniak, Description of the D*(s)(2320) resonance as the D pi atom, Phys. Lett. B567 (2003) 23-26. arXiv:hep-ph/0305060, doi:10.1016/S0370-2693(03)00865-7.

[54] D. Besson, et al., Observation of a narrow resonance of mass $2.46 \mathrm{GeV} / \mathrm{c}^{2}$ decaying to $D_{s}^{*} \pi^{0}$ and confirmation of the $D_{s J}^{*}(2317)$ state, Phys. Rev. D68 (2003) 032002, [Erratum: Phys. Rev. D75, 119908 (2007)]. arXiv:hep-ex/0305100, doi:10.1103/PhysRevD.68.032002,10.1103/ PhysRevD.75.119908. 
[55] E. van Beveren, G. Rupp, Observed D(s)(2317) and tentative D(2030) as the charmed cousins of the light scalar nonet, Phys. Rev. Lett. 91 (2003) 012003. arXiv:hep-ph/0305035, doi: 10.1103/PhysRevLett.91.012003.

[56] Y.-B. Dai, X.-Q. Li, S.-L. Zhu, Y.-B. Zuo, Contribution of DK continuum in the QCD sum rule for D(sJ) (2317), Eur. Phys. J. C55 (2008) 249-258. arXiv:hep-ph/0610327, doi:10.1140/epjc/ s10052-008-0591-9.

[57] S. K. Choi, et al., Observation of a narrow charmonium-like state in exclusive $B^{ \pm} \rightarrow K^{ \pm} \pi^{+} \pi^{-} J / \psi$ decays, Phys. Rev. Lett. 91 (2003) 262001. arXiv:hep-ex/0309032, doi:10.1103/PhysRevLett. 91.262001.

[58] C. Meng, K.-T. Chao, Decays of the $X(3872)$ and $\chi_{c 1}(2 P)$ charmonium, Phys. Rev. D75 (2007) 114002. arXiv:hep-ph/0703205, doi:10.1103/PhysRevD.75.114002.

[59] R. Aaij, et al., Amplitude analysis of $B^{+} \rightarrow J / \psi \phi K^{+}$decays, Phys. Rev. D95 (1) (2017) 012002. arXiv:1606.07898, doi:10.1103/PhysRevD.95.012002.

[60] R. Aaij, et al., Observation of $J / \psi \phi$ structures consistent with exotic states from amplitude analysis of $B^{+} \rightarrow J / \psi \phi K^{+}$decays, Phys. Rev. Lett. 118 (2) (2017) 022003. arXiv:1606.07895, doi:10.1103/PhysRevLett.118.022003.

[61] T. Aaltonen, et al., Observation of the $Y(4140)$ structure in the $J / \psi \phi$ mass spectrum in $B^{ \pm} \rightarrow$ $J / \psi \phi K^{ \pm}$decays, Mod. Phys. Lett. A32 (26) (2017) 1750139. arXiv:1101.6058, doi:10.1142/ S0217732317501395.

[62] S. Chatrchyan, et al., Observation of a peaking structure in the $J / \psi \phi$ mass spectrum from $B^{ \pm} \rightarrow J / \psi \phi K^{ \pm}$decays, Phys. Lett. B734 (2014) 261-281. arXiv:1309.6920, doi:10.1016/ j.physletb.2014.05.055.

[63] M. Ablikim, et al., Precise measurement of the $e^{+} e^{-} \rightarrow \pi^{+} \pi^{-} J / \psi$ cross section at center-of-mass energies from 3.77 to $4.60 \mathrm{GeV}$, Phys. Rev. Lett. 118 (9) (2017) 092001. arXiv:1611.01317, doi:10.1103/PhysRevLett.118.092001.

[64] M. Ablikim, et al., Evidence of Two Resonant Structures in $e^{+} e^{-} \rightarrow \pi^{+} \pi^{-} h_{c}$, Phys. Rev. Lett. 118 (9) (2017) 092002. arXiv:1610.07044, doi:10.1103/PhysRevLett.118.092002.

[65] K. Chilikin, et al., Observation of an alternative $\chi_{c 0}(2 P)$ candidate in $e^{+} e^{-} \rightarrow J / \psi D \bar{D}$, Phys. Rev. D95 (2017) 112003. arXiv:1704.01872, doi:10.1103/PhysRevD.95.112003.

[66] M. Ablikim, et al., Measurement of $e^{+} e^{-} \rightarrow \pi^{+} \pi^{-} \psi(3686)$ from 4.008 to $4.600 \mathrm{GeV}$ and observation of a charged structure in the $\pi^{ \pm} \psi(3686)$ mass spectrum, Phys. Rev. D96 (3) (2017) 032004, [erratum: Phys. Rev.D99,no.1,019903(2019)]. arXiv:1703.08787, doi:10.1103/PhysRevD.96. 032004, 10.1103/PhysRevD.99.019903.

[67] R. Aaij, et al., Evidence for an $\eta_{c}(1 S) \pi^{-}$resonance in $B^{0} \rightarrow \eta_{c}(1 S) K^{+} \pi^{-}$decays, Eur. Phys. J. C78 (12) (2018) 1019. arXiv:1809.07416, doi:10.1140/epjc/s10052-018-6447-z.

[68] Talk given by T. Skwarnicki, on behalf of the LHCb Collaboration at Moriond2019.

[69] A. Hosaka, T. Iijima, K. Miyabayashi, Y. Sakai, S. Yasui, Exotic hadrons with heavy flavors: X, Y, Z, and related states, PTEP 2016 (6) (2016) 062C01. arXiv:1603.09229, doi:10.1093/ptep/ ptw045. 
[70] A. Ali, J. S. Lange, S. Stone, Exotics: Heavy Pentaquarks and Tetraquarks, Prog. Part. Nucl. Phys. 97 (2017) 123-198. arXiv:1706.00610, doi:10.1016/j.ppnp.2017.08.003.

[71] M. Karliner, J. L. Rosner, T. Skwarnicki, Multiquark States, Ann. Rev. Nucl. Part. Sci. 68 (2018) 17-44. arXiv:1711.10626, doi:10.1146/annurev-nucl-101917-020902.

[72] F.-K. Guo, C. Hanhart, U.-G. Meissner, Q. Wang, Q. Zhao, B.-S. Zou, Hadronic molecules, Rev. Mod. Phys. 90 (1) (2018) 015004. arXiv:1705.00141, doi:10.1103/RevModPhys.90.015004.

[73] A. Esposito, A. Pilloni, A. D. Polosa, Multiquark Resonances, Phys. Rept. 668 (2016) 1-97. arXiv:1611.07920, doi:10.1016/j.physrep.2016.11.002.

[74] R. F. Lebed, R. E. Mitchell, E. S. Swanson, Heavy-Quark QCD Exotica, Prog. Part. Nucl. Phys. 93 (2017) 143-194. arXiv:1610.04528, doi:10.1016/j.ppnp.2016.11.003.

[75] J.-M. Richard, Exotic hadrons: review and perspectives, Few Body Syst. 57 (12) (2016) 11851212. arXiv:1606.08593, doi:10.1007/s00601-016-1159-0.

[76] S. L. Olsen, T. Skwarnicki, D. Zieminska, Nonstandard heavy mesons and baryons: Experimental evidence, Rev. Mod. Phys. 90 (1) (2018) 015003. arXiv:1708.04012, doi:10.1103/RevModPhys. 90.015003 .

[77] B. Silvestre-Brac, Systematics of $\mathrm{Q}^{* * 2}$ (anti-Q**2) systems with a chromomagnetic interaction, Phys. Rev. D46 (1992) 2179-2189. doi:10.1103/PhysRevD.46.2179.

[78] B. Keren-Zur, Testing confining potentials through meson/baryon hyperfine splitting ratio, Annals Phys. 323 (2008) 631-642. arXiv:hep-ph/0703011, doi:10.1016/j.aop.2007.04.010.

[79] A. De Rujula, H. Georgi, S. L. Glashow, Hadron Masses in a Gauge Theory, Phys. Rev. D12 (1975) 147-162. doi:10.1103/PhysRevD.12.147.

[80] A. D. Sakharov, Ya. B. Zel'dovich, Kvarkovaia struktura i massy sil'novzaimodeistvuyushchikh chastits, Yad. Fiz. 4 (1966) 395-400.

[81] M. Oka, Origin of the short-range part of generalized two- and three-body nuclear force, Nucl. Phys. A881 (2012) 6-13. doi:10.1016/j.nuclphysa.2012.01.007.

[82] S. Maeda, M. Oka, A. Yokota, E. Hiyama, Y.-R. Liu, A model of charmed baryonCnucleon potential and two- and three-body bound states with charmed baryon, PTEP 2016 (2) (2016) 023D02. arXiv:1509.02445, doi:10.1093/ptep/ptv194.

[83] R. L. Jaffe, Multi-Quark Hadrons. 2. Methods, Phys. Rev. D15 (1977) 281. doi:10.1103/ PhysRevD. 15.281.

[84] F. Buccella, H. Hogaasen, J.-M. Richard, P. Sorba, Chromomagnetism, flavour symmetry breaking and S-wave tetraquarks, Eur. Phys. J. C49 (2007) 743-754. arXiv:hep-ph/0608001, doi:10. 1140/epjc/s10052-006-0142-1.

[85] A. T. M. Aerts, P. J. G. Mulders, J. J. de Swart, Multi-Baryon States in the Bag Model, Phys. Rev. D17 (1978) 260. doi:10.1103/PhysRevD.17.260.

[86] H. Hogaasen, P. Sorba, The Systematics of Possibly Narrow Quark States with Baryon Number One, Nucl. Phys. B145 (1978) 119-140. doi:10.1016/0550-3213(78)90417-0. 
[87] Y. Cui, X.-L. Chen, W.-Z. Deng, S.-L. Zhu, The J**P = 1+ ud anti-s anti-s tetraquark, Phys. Rev. D73 (2006) 014018. arXiv:hep-ph/0511150, doi:10.1103/PhysRevD.73.014018.

[88] Y. Cui, X.-L. Chen, W.-Z. Deng, S.-L. Zhu, The Possible Heavy Tetraquarks qQ anti-q anti-Q, qq anti-Q anti-Q and qQ anti-Q anti-Q, HEPNP 31 (2007) 7-13. arXiv:hep-ph/0607226.

[89] H. Hogaasen, P. Sorba, The Colour triplet qq anti-q cluster and pentaquark models, Mod. Phys. Lett. A19 (2004) 2403-2410. arXiv:hep-ph/0406078, doi:10.1142/S0217732304015592.

[90] L. Maiani, F. Piccinini, A. D. Polosa, V. Riquer, A New look at scalar mesons, Phys. Rev. Lett. 93 (2004) 212002. arXiv:hep-ph/0407017, doi:10.1103/PhysRevLett.93.212002.

[91] H. J. Lipkin, Are There Charmed - Strange Exotic Mesons?, Phys. Lett. 70B (1977) 113-116. doi : 10.1016/0370-2693(77)90357-4.

[92] M. Anselmino, E. Predazzi, S. Ekelin, S. Fredriksson, D. B. Lichtenberg, Diquarks, Rev. Mod. Phys. 65 (1993) 1199-1234. doi:10.1103/RevModPhys.65.1199.

[93] S. J. Brodsky, D. S. Hwang, R. F. Lebed, Dynamical Picture for the Formation and Decay of the Exotic XYZ Mesons, Phys. Rev. Lett. 113 (11) (2014) 112001. arXiv:1406.7281, doi: 10.1103/PhysRevLett.113.112001.

[94] L. Maiani, A. D. Polosa, V. Riquer, A Theory of X and Z Multiquark Resonances, Phys. Lett. B778 (2018) 247-251. arXiv:1712.05296, doi:10.1016/j.physletb.2018.01.039.

[95] L. Maiani, F. Piccinini, A. D. Polosa, V. Riquer, Diquark-antidiquarks with hidden or open charm and the nature of X(3872), Phys. Rev. D71 (2005) 014028. arXiv:hep-ph/0412098, doi:10.1103/PhysRevD.71.014028.

[96] N. V. Drenska, R. Faccini, A. D. Polosa, Exotic Hadrons with Hidden Charm and Strangeness, Phys. Rev. D79 (2009) 077502. arXiv:0902.2803, doi:10.1103/PhysRevD.79.077502.

[97] L. Maiani, F. Piccinini, A. D. Polosa, V. Riquer, The $Z(4430)$ and a New Paradigm for Spin Interactions in Tetraquarks, Phys. Rev. D89 (2014) 114010. arXiv:1405.1551, doi:10.1103/ PhysRevD .89.114010.

[98] R. F. Lebed, The Pentaquark Candidates in the Dynamical Diquark Picture, Phys. Lett. B749 (2015) 454-457. arXiv:1507.05867, doi:10.1016/j.physletb.2015.08.032.

[99] M. Karliner, H. J. Lipkin, A Diquark - triquark model for the K N pentaquark, Phys. Lett. B575 (2003) 249-255. arXiv:hep-ph/0402260, doi:10.1016/j.physletb.2003.09.062.

[100] R. Zhu, C.-F. Qiao, Pentaquark states in a diquarkCtriquark model, Phys. Lett. B756 (2016) 259-264. arXiv:1510.08693, doi:10.1016/j.physletb.2016.03.022.

[101] H. Høgaasen, E. Kou, J.-M. Richard, P. Sorba, Isovector and hidden-beauty partners of the X(3872), Phys. Lett. B732 (2014) 97-100. arXiv:1309.2049, doi:10.1016/j.physletb. 2014. 03.027.

[102] X.-Z. Weng, X.-L. Chen, W.-Z. Deng, Masses of doubly heavy-quark baryons in an extended chromomagnetic model, Phys. Rev. D97 (5) (2018) 054008. arXiv:1801.08644, doi:10.1103/ PhysRevD .97.054008.

[103] G. Rossi, G. Veneziano, The string-junction picture of multiquark states: an update, JHEP 06 (2016) 041. arXiv:1603.05830, doi:10.1007/JHEP06 (2016) 041. 
[104] M. Karliner, J. L. Rosner, Baryons with two heavy quarks: Masses, production, decays, and detection, Phys. Rev. D90 (9) (2014) 094007. arXiv:1408.5877, doi:10.1103/PhysRevD.90. 094007.

[105] M. Karliner, S. Nussinov, J. L. Rosner, $Q Q \bar{Q} \bar{Q}$ states: masses, production, and decays, Phys. Rev. D95 (3) (2017) 034011. arXiv:1611.00348, doi:10.1103/PhysRevD.95.034011.

[106] J. Wu, Y.-R. Liu, K. Chen, X. Liu, S.-L. Zhu, X(4140), X(4270), X(4500) and $X(4700)$ and their $\operatorname{cs} \bar{c} \bar{s}$ tetraquark partners, Phys. Rev. D94 (9) (2016) 094031. arXiv:1608.07900, doi: 10.1103/PhysRevD.94.094031.

[107] K. Chen, X. Liu, J. Wu, Y.-R. Liu, S.-L. Zhu, Triply heavy tetraquark states with the $Q Q \bar{Q} \bar{q}$ configuration, Eur. Phys. J. A53 (1) (2017) 5. arXiv:1609.06117, doi:10.1140/epja/ i2017-12199-3.

[108] J. Wu, Y.-R. Liu, K. Chen, X. Liu, S.-L. Zhu, Hidden-charm pentaquarks and their hiddenbottom and $B_{c}$-like partner states, Phys. Rev. D95 (3) (2017) 034002. arXiv:1701.03873, doi: 10.1103/PhysRevD.95.034002.

[109] S.-Q. Luo, K. Chen, X. Liu, Y.-R. Liu, S.-L. Zhu, Exotic tetraquark states with the $q q \bar{Q} \bar{Q}$ configuration, Eur. Phys. J. C77 (10) (2017) 709. arXiv:1707.01180, doi:10.1140/epjc/ s10052-017-5297-4.

[110] J. Wu, Y.-R. Liu, K. Chen, X. Liu, S.-L. Zhu, Heavy-flavored tetraquark states with the $Q Q \bar{Q} \bar{Q}$ configuration, Phys. Rev. D97 (9) (2018) 094015. arXiv:1605.01134, doi:10.1103/PhysRevD. 97.094015 .

[111] Q.-S. Zhou, K. Chen, X. Liu, Y.-R. Liu, S.-L. Zhu, Surveying exotic pentaquarks with the typical

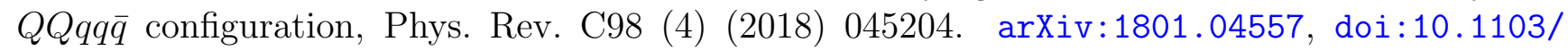
PhysRevC.98.045204.

[112] S.-Y. Li, Y.-R. Liu, Y.-N. Liu, Z.-G. Si, J. Wu, Pentaquark states with the $Q Q Q q \bar{q}$ configuration in a simple model, Eur. Phys. J. C79 (1) (2019) 87. arXiv:1809.08072, doi:10.1140/epjc/ s10052-019-6589-7.

[113] J. Wu, X. Liu, Y.-R. Liu, S.-L. Zhu, Systematic studies of charmonium-, bottomonium-, and $B_{c}$-like tetraquark states, Phys. Rev. D99 (1) (2019) 014037. arXiv:1810.06886, doi:10.1103/ PhysRevD.99.014037.

[114] F. Stancu, Can Y(4140) be a c anti-c s anti-s tetraquark?, J. Phys. G37 (2010) 075017. arXiv: 0906.2485, doi:10.1088/0954-3899/37/7/075017.

[115] J. Leandri, B. Silvestre-Brac, Systematics of $\bar{Q} Q^{-} 4$ Systems With a Pure Chromomagnetic Interaction, Phys. Rev. D40 (1989) 2340-2352. doi:10.1103/PhysRevD.40.2340.

[116] B. Silvestre-Brac, J. Leandri, Systematics of q-6 systems in a simple chromomagnetic model, Phys. Rev. D45 (1992) 4221-4239. doi:10.1103/PhysRevD.45.4221.

[117] J. Vijande, A. Valcarce, J.-M. Richard, Adiabaticity and color mixing in tetraquark spectroscopy, Phys. Rev. D87 (3) (2013) 034040. arXiv:1301.6212, doi:10.1103/PhysRevD.87.034040.

[118] H. J. Lipkin, Relations Between Meson and Baryon Hyperfine Splittings, Phys. Lett. B171 (1986) 293-294. doi:10.1016/0370-2693(86)91551-0. 
[119] N. Mathur, M. Padmanath, S. Mondal, Precise predictions of charmed-bottom hadrons from lattice QCD, Phys. Rev. Lett. 121 (20) (2018) 202002. arXiv:1806.04151, doi:10.1103/ PhysRevLett.121.202002.

[120] N. Mathur, M. Padmanath, Lattice QCD study of doubly-charmed strange baryons, Phys. Rev. D99 (3) (2019) 031501. arXiv:1807.00174, doi:10.1103/PhysRevD.99.031501.

[121] L. Maiani, V. Riquer, F. Piccinini, A. D. Polosa, Four quark interpretation of Y(4260), Phys. Rev. D72 (2005) 031502. arXiv:hep-ph/0507062, doi:10.1103/PhysRevD.72.031502.

[122] L. Maiani, A. D. Polosa, V. Riquer, The Charged Z(4433): Towards a new spectroscopy, arXiv: 0708.3997.

[123] L. Maiani, A. D. Polosa, V. Riquer, The charged Z(4430) in the diquark-antidiquark picture, New J. Phys. 10 (2008) 073004. doi:10.1088/1367-2630/10/7/073004.

[124] H. Hogaasen, J. M. Richard, P. Sorba, A Chromomagnetic mechanism for the X(3872) resonance, Phys. Rev. D73 (2006) 054013. arXiv:hep-ph/0511039, doi:10.1103/PhysRevD.73.054013.

[125] M. Abud, F. Buccella, F. Tramontano, Hints for the existence of hexaquark states in the baryonantibaryon sector, Phys. Rev. D81 (2010) 074018. arXiv:0912.4299, doi:10.1103/PhysRevD. 81.074018.

[126] T. Guo, L. Cao, M.-Z. Zhou, H. Chen, The Possible candidates of tetraquark : $Z_{b}(10610)$ and $Z_{b}(10650)$ arXiv:1106.2284.

[127] A. Ali, C. Hambrock, W. Wang, Tetraquark Interpretation of the Charged Bottomonium-like states $Z_{b}^{+-}(10610)$ and $Z_{b}^{+-}(10650)$ and Implications, Phys. Rev. D85 (2012) 054011. arXiv: 1110.1333, doi:10.1103/PhysRevD.85.054011.

[128] A. Ali, L. Maiani, A. D. Polosa, V. Riquer, Hidden-Beauty Charged Tetraquarks and Heavy Quark Spin Conservation, Phys. Rev. D91 (1) (2015) 017502. arXiv:1412.2049, doi:10.1103/ PhysRevD.91.017502.

[129] A. Ali, C. Hambrock, I. Ahmed, M. J. Aslam, A case for hidden $b \bar{b}$ tetraquarks based on $e^{+} e^{-} \rightarrow b \bar{b}$ cross section between $\sqrt{s}=10.54$ and $11.20 \mathrm{GeV}$, Phys. Lett. B684 (2010) 28-39. arXiv:0911. 2787, doi:10.1016/j.physletb.2009.12.053.

[130] A. Ali, C. Hambrock, M. J. Aslam, A Tetraquark interpretation of the BELLE data on the anomalous $\Upsilon(1 S) \pi^{+} \pi^{-}$and $\Upsilon(2 S) \pi^{+} \pi^{-}$production near the $\Upsilon(5 S)$ resonance, Phys. Rev. Lett. 104 (2010) 162001, [Erratum: Phys. Rev. Lett. 107, 049903 (2011)]. arXiv:0912.5016, doi: 10.1103/PhysRevLett.104.162001,10.1103/PhysRevLett.107.049903.

[131] L. Maiani, V. Riquer, R. Faccini, F. Piccinini, A. Pilloni, A. D. Polosa, A $J^{P G}=1^{++}$Charged Resonance in the $Y(4260) \rightarrow \pi^{+} \pi^{-} J / \psi$ Decay?, Phys. Rev. D87 (11) (2013) 111102. arXiv: 1303.6857, doi:10.1103/PhysRevD.87.111102.

[132] H. X. Chen, L. Maiani, A. D. Polosa, V. Riquer, $Y(4260) \rightarrow \gamma+X(3872)$ in the diquarkonium picture, Eur. Phys. J. C75 (11) (2015) 550. arXiv:1510.03626, doi:10.1140/epjc/ s10052-015-3781-2.

[133] V. V. Anisovich, M. A. Matveev, A. V. Sarantsev, A. N. Semenova, Exotic mesons with hidden charm as diquark-antidiquark states, Int. J. Mod. Phys. A30 (2015) 1550186. arXiv:1507.07232, doi:10.1142/S0217751X15501869. 
[134] H. Kim, K. S. Kim, M.-K. Cheoun, D. Jido, M. Oka, Testing the tetraquark structure for the $\mathrm{X}$ resonances in the low-lying region, Eur. Phys. J. A52 (7) (2016) 184. arXiv:1602.07540, doi : 10.1140/epja/i2016-16184-0.

[135] R. F. Lebed, A. D. Polosa, $\chi_{c 0}(3915)$ As the Lightest $c \bar{c} s \bar{s}$ State, Phys. Rev. D93 (9) (2016) 094024. arXiv:1602.08421, doi:10.1103/PhysRevD.93.094024.

[136] L. Maiani, A. D. Polosa, V. Riquer, Interpretation of Axial Resonances in J/psi-phi at LHCb, Phys. Rev. D94 (5) (2016) 054026. arXiv:1607.02405, doi:10.1103/PhysRevD.94.054026.

[137] R. Zhu, Hidden charm octet tetraquarks from a diquark-antidiquark model, Phys. Rev. D94 (5) (2016) 054009. arXiv:1607.02799, doi:10.1103/PhysRevD.94.054009.

[138] A. Ali, L. Maiani, A. V. Borisov, I. Ahmed, M. Jamil Aslam, A. Ya. Parkhomenko, A. D. Polosa, A. Rehman, A new look at the Y tetraquarks and $\Omega_{c}$ baryons in the diquark model, Eur. Phys. J. C78 (1) (2018) 29. arXiv:1708.04650, doi:10.1140/epjc/s10052-017-5501-6.

[139] C. P. Shen, et al., Evidence for a new resonance and search for the $Y(4140)$ in the $\gamma \gamma \rightarrow \phi J / \psi$ process, Phys. Rev. Lett. 104 (2010) 112004. arXiv:0912.2383, doi:10.1103/PhysRevLett. 104.112004 .

[140] J. l. Ballot, J. M. Richard, FOUR QUARK STATES IN ADDITIVE POTENTIALS, Phys. Lett. 123B (1983) 449-451. doi:10.1016/0370-2693(83)90991-7.

[141] H. J. Lipkin, A MODEL INDEPENDENT APPROACH TO MULTI - QUARK BOUND STATES, Phys. Lett. B172 (1986) 242-247. doi:10.1016/0370-2693(86)90843-9.

[142] S. Zouzou, B. Silvestre-Brac, C. Gignoux, J. M. Richard, FOUR QUARK BOUND STATES, Z. Phys. C30 (1986) 457. doi:10.1007/BF01557611.

[143] L. Heller, J. A. Tjon, On the Existence of Stable Dimesons, Phys. Rev. D35 (1987) 969. doi: 10.1103/PhysRevD.35.969.

[144] J. Carlson, L. Heller, J. A. Tjon, Stability of Dimesons, Phys. Rev. D37 (1988) 744. doi: 10.1103/PhysRevD.37.744.

[145] A. V. Manohar, M. B. Wise, Exotic Q Q anti-q anti-q states in QCD, Nucl. Phys. B399 (1993) 17-33. arXiv:hep-ph/9212236, doi:10.1016/0550-3213(93)90614-U.

[146] T. E. O. Ericson, G. Karl, Strength of pion exchange in hadronic molecules, Phys. Lett. B309 (1993) 426-430. doi:10.1016/0370-2693(93)90957-J.

[147] B. Silvestre-Brac, C. Semay, Spectrum and decay properties of diquonia, Z. Phys. C59 (1993) 457-470. doi:10.1007/BF01498626.

[148] B. Silvestre-Brac, C. Semay, Systematics of L =0 q-2 anti-q-2 systems, Z. Phys. C57 (1993) 273-282. doi:10.1007/BF01565058.

[149] C. Semay, B. Silvestre-Brac, Diquonia and potential models, Z. Phys. C61 (1994) 271-275. doi: 10.1007/BF01413104.

[150] M. A. Moinester, How to search for doubly charmed baryons and tetraquarks, Z. Phys. A355 (1996) 349-362. arXiv:hep-ph/9506405, doi:10.1007/s002180050123. 
[151] S. Pepin, F. Stancu, M. Genovese, J. M. Richard, Tetraquarks with color blind forces in chiral quark models, Phys. Lett. B393 (1997) 119-123. arXiv:hep-ph/9609348, doi:10.1016/ S0370-2693(96)01597-3.

[152] D. M. Brink, F. Stancu, Tetraquarks with heavy flavors, Phys. Rev. D57 (1998) 6778-6787. doi:10.1103/PhysRevD.57.6778.

[153] J. Schaffner-Bielich, A. P. Vischer, Charmlets, Phys. Rev. D57 (1998) 4142-4153. arXiv:nucl-th/ 9710064, doi:10.1103/PhysRevD.57.4142.

[154] B. A. Gelman, S. Nussinov, Does a narrow tetraquark cc anti-u anti-d state exist?, Phys. Lett. B551 (2003) 296-304. arXiv:hep-ph/0209095, doi:10.1016/S0370-2693(02)03069-1.

[155] C. Stewart, R. Koniuk, Hadronic molecules in lattice QCD, Phys. Rev. D57 (1998) 5581-5585. arXiv:hep-lat/9803003, doi:10.1103/PhysRevD.57.5581.

[156] C. Michael, P. Pennanen, Two heavy - light mesons on a lattice, Phys. Rev. D60 (1999) 054012. arXiv:hep-lat/9901007, doi:10.1103/PhysRevD.60.054012.

[157] T. Barnes, N. Black, D. J. Dean, E. S. Swanson, B B intermeson potentials in the quark model, Phys. Rev. C60 (1999) 045202. arXiv:nucl-th/9902068, doi:10.1103/PhysRevC.60.045202.

[158] M. S. Cook, H. R. Fiebig, A Lattice study of interaction mechanisms in a heavy light meson meson systemarXiv:hep-lat/0210054.

[159] S. H. Lee, S. Yasui, W. Liu, C. M. Ko, Charmed exotics in Heavy Ion Collisions, Eur. Phys. J. C54 (2008) 259-265. arXiv:0707.1747, doi:10.1140/epjc/s10052-007-0516-z.

[160] S. H. Lee, S. Yasui, Stable multiquark states with heavy quarks in a diquark model, Eur. Phys. J. C64 (2009) 283-295. arXiv:0901.2977, doi:10.1140/epjc/s10052-009-1140-x.

[161] T. Hyodo, Y.-R. Liu, M. Oka, K. Sudoh, S. Yasui, Production of doubly charmed tetraquarks with exotic color configurations in electron-positron collisions, Phys. Lett. B721 (2013) 56-60. arXiv:1209.6207, doi:10.1016/j.physletb.2013.02.045.

[162] T. Hyodo, Y.-R. Liu, M. Oka, S. Yasui, Spectroscopy and production of doubly charmed tetraquarksarXiv: 1708.05169.

[163] D. Ebert, R. N. Faustov, V. O. Galkin, W. Lucha, Masses of tetraquarks with two heavy quarks in the relativistic quark model, Phys. Rev. D76 (2007) 114015. arXiv:0706.3853, doi:10.1103/ PhysRevD.76.114015.

[164] E. J. Eichten, C. Quigg, Heavy-quark symmetry implies stable heavy tetraquark mesons $Q_{i} Q_{j} \bar{q}_{k} \bar{q}_{l}$, Phys. Rev. Lett. 119 (20) (2017) 202002. arXiv:1707.09575, doi:10.1103/PhysRevLett.119. 202002.

[165] T. Mehen, Implications of Heavy Quark-Diquark Symmetry for Excited Doubly Heavy Baryons and Tetraquarks, Phys. Rev. D96 (9) (2017) 094028. arXiv:1708.05020, doi:10.1103/ PhysRevD.96.094028.

[166] M. Karliner, J. L. Rosner, Discovery of doubly-charmed $\Xi_{c c}$ baryon implies a stable $(b b \bar{u} \bar{d})$ tetraquark, Phys. Rev. Lett. 119 (20) (2017) 202001. arXiv:1707.07666, doi:10.1103/ PhysRevLett.119.202001. 
[167] X. Yan, B. Zhong, R. Zhu, Doubly charmed tetraquarks in a diquarkCantidiquark model, Int. J. Mod. Phys. A33 (16) (2018) 1850096. arXiv:1804.06761, doi:10.1142/S0217751X18500963.

[168] Y. Xing, R. Zhu, Weak Decays of Stable Doubly Heavy Tetraquark States, Phys. Rev. D98 (5) (2018) 053005. arXiv:1806.01659, doi:10.1103/PhysRevD.98.053005.

[169] Y. Iwasaki, A Possible Model for New Resonances-Exotics and Hidden Charm, Prog. Theor. Phys. 54 (1975) 492. doi:10.1143/PTP.54.492.

[170] Y. Iwasaki, Is a State c anti-c c anti-c Found at 6.0-GeV?, Phys. Rev. Lett. 36 (1976) 1266. doi:10.1103/PhysRevLett.36.1266.

[171] Y. Iwasaki, How to Find eta(C) and a Possible State Charm anti-Charm Charm anti-Charm, Phys. Rev. D16 (1977) 220. doi:10.1103/PhysRevD.16.220.

[172] K.-T. Chao, The (c c) - (anti-c anti-c) (Diquark - anti-Diquark) States in e+ e- Annihilation, Z. Phys. C7 (1981) 317. doi:10.1007/BF01431564.

[173] J. P. Ader, J. M. Richard, P. Taxil, DO NARROW HEAVY MULTI - QUARK STATES EXIST?, Phys. Rev. D25 (1982) 2370. doi:10.1103/PhysRevD.25.2370.

[174] L. Heller, J. A. Tjon, On Bound States of Heavy $Q^{2} \bar{Q}^{2}$ Systems, Phys. Rev. D32 (1985) 755. doi:10.1103/PhysRevD.32.755.

[175] A. M. Badalian, B. L. Ioffe, A. V. Smilga, FOUR QUARK STATES IN THE HEAVY QUARK SYSTEM, Nucl. Phys. B281 (1987) 85. doi:10.1016/0550-3213(87)90248-3.

[176] Yu. S. Kalashnikova, I. M. Narodetsky, DISSOCIATION CALCULATIONS FOR S WAVE Q**2 anti-Q**2 SYSTEMS, Z. Phys. C43 (1989) 273. doi:10.1007/BF01588215.

[177] A. V. Berezhnoy, A. K. Likhoded, A. V. Luchinsky, A. A. Novoselov, Double J/psi-meson Production at LHC and 4c-tetraquark state, Phys. Rev. D84 (2011) 094023. arXiv:1101.5881, doi:10.1103/PhysRevD.84.094023.

[178] A. V. Berezhnoy, A. V. Luchinsky, A. A. Novoselov, Tetraquarks Composed of 4 Heavy Quarks, Phys. Rev. D86 (2012) 034004. arXiv:1111.1867, doi:10.1103/PhysRevD.86.034004.

[179] V. V. Kiselev, A. K. Likhoded, O. N. Pakhomova, V. A. Saleev, Mass spectra of doubly heavy Omega $Q Q^{\prime}$ baryons, Phys. Rev. D66 (2002) 034030. arXiv:hep-ph/0206140, doi:10.1103/ PhysRevD.66.034030.

[180] M.-S. Liu, Q.-F. L, X.-H. Zhong, Q. Zhao, Fully-heavy tetraquarksarXiv:1901.02564.

[181] R. Aaij, et al., Search for beautiful tetraquarks in the $\Upsilon(1 S) \mu^{+} \mu^{-}$invariant-mass spectrum, JHEP 10 (2018) 086. arXiv:1806.09707, doi:10.1007/JHEP10(2018)086.

[182] V. Khachatryan, et al., Observation of $\Upsilon(1 \mathrm{~S})$ pair production in proton-proton collisions at $\sqrt{s}=8$ TeV, JHEP 05 (2017) 013. arXiv:1610.07095, doi:10.1007/JHEP05(2017)013.

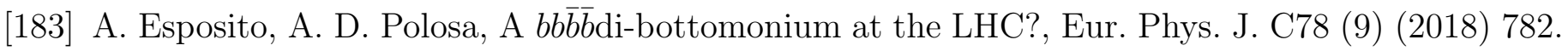
arXiv:1807.06040, doi:10.1140/epjc/s10052-018-6269-z.

[184] J.-J. Wu, R. Molina, E. Oset, B. S. Zou, Prediction of narrow $N^{*}$ and $\Lambda^{*}$ resonances with hidden charm above $4 \mathrm{GeV}$, Phys. Rev. Lett. 105 (2010) 232001. arXiv:1007.0573, doi:10.1103/ PhysRevLett.105.232001. 
[185] R. Aaij, et al., Observation of $J / \psi p$ Resonances Consistent with Pentaquark States in $\Lambda_{b}^{0} \rightarrow$ $J / \psi K^{-} p$ Decays, Phys. Rev. Lett. 115 (2015) 072001. arXiv:1507.03414, doi:10.1103/ PhysRevLett.115.072001.

[186] R. Aaij, et al., Model-independent evidence for $J / \psi p$ contributions to $\Lambda_{b}^{0} \rightarrow J / \psi p K^{-}$decays, Phys. Rev. Lett. 117 (8) (2016) 082002. arXiv:1604.05708, doi:10.1103/PhysRevLett.117. 082002.

[187] R. Aaij, et al., Evidence for exotic hadron contributions to $\Lambda_{b}^{0} \rightarrow J / \psi p \pi^{-}$decays, Phys. Rev. Lett. 117 (8) (2016) 082003, [Addendum: Phys. Rev. Lett.118,119901(2017)]. arXiv: 1606.06999, doi:10.1103/PhysRevLett.118.119901, 10.1103/PhysRevLett.117.082003, 10. 1103/PhysRevLett.117.109902.

[188] A. N. Hiller Blin, C. Fernndez-Ramrez, A. Jackura, V. Mathieu, V. I. Mokeev, A. Pilloni, A. P. Szczepaniak, Studying the $\mathrm{P}_{c}(4450)$ resonance in $\mathrm{J} / \psi$ photoproduction off protons, Phys. Rev. D94 (3) (2016) 034002. arXiv:1606.08912, doi:10.1103/PhysRevD.94.034002.

[189] V. Kubarovsky, M. B. Voloshin, Search for Hidden-Charm Pentaquark with CLAS12arXiv: 1609. 00050.

[190] Z. E. Meziani, et al., A Search for the LHCb Charmed 'Pentaquark' using Photo-Production of $J / \psi$ at Threshold in Hall C at Jefferson LabarXiv:1609.00676.

[191] S. Joosten, Z. E. Meziani, Heavy Quarkonium Production at Threshold: from JLab to EIC, PoS QCDEV2017 (2018) 017. arXiv:1802.02616, doi:10.22323/1.308.0017.

[192] M. F. M. Lutz, et al., Physics Performance Report for PANDA: Strong Interaction Studies with Antiprotons, arXiv:0903.3905.

[193] J.-J. Wu, R. Molina, E. Oset, B. S. Zou, Dynamically generated $N^{*}$ and $\Lambda^{*}$ resonances in the hidden charm sector around 4.3 GeV, Phys. Rev. C84 (2011) 015202. arXiv:1011.2399, doi: 10.1103/PhysRevC.84.015202.

[194] W. L. Wang, F. Huang, Z. Y. Zhang, B. S. Zou, $\Sigma_{c} \bar{D}$ and $\Lambda_{c} \bar{D}$ states in a chiral quark model, Phys. Rev. C84 (2011) 015203. arXiv:1101.0453, doi:10.1103/PhysRevC.84.015203.

[195] Z.-C. Yang, Z.-F. Sun, J. He, X. Liu, S.-L. Zhu, The possible hidden-charm molecular baryons composed of anti-charmed meson and charmed baryon, Chin. Phys. C36 (2012) 6-13. arXiv: 1105.2901, doi:10.1088/1674-1137/36/1/002.

[196] S. G. Yuan, K. W. Wei, J. He, H. S. Xu, B. S. Zou, Study of $q q q c \bar{c}$ five quark system with three kinds of quark-quark hyperfine interaction, Eur. Phys. J. A48 (2012) 61. arXiv:1201.0807, doi:10.1140/epja/i2012-12061-2.

[197] J.-J. Wu, T. S. H. Lee, B. S. Zou, Nucleon Resonances with Hidden Charm in Coupled-Channel Models, Phys. Rev. C85 (2012) 044002. arXiv:1202.1036, doi:10.1103/PhysRevC.85.044002.

[198] C. Garcia-Recio, J. Nieves, O. Romanets, L. L. Salcedo, L. Tolos, Hidden charm $N$ and $\Delta$ resonances with heavy-quark symmetry, Phys. Rev. D87 (2013) 074034. arXiv:1302.6938, doi:10.1103/PhysRevD.87.074034.

[199] C. W. Xiao, J. Nieves, E. Oset, Combining heavy quark spin and local hidden gauge symmetries in the dynamical generation of hidden charm baryons, Phys. Rev. D88 (2013) 056012. arXiv: 1304.5368, doi:10.1103/PhysRevD.88.056012. 
[200] Y. Huang, J. He, H.-F. Zhang, X.-R. Chen, Discovery potential of hidden charm baryon resonances via photoproduction, J. Phys. G41 (11) (2014) 115004. arXiv:1305.4434, doi: 10.1088/0954-3899/41/11/115004.

[201] X.-Q. Li, X. Liu, A possible global group structure for exotic states, Eur. Phys. J. C74 (12) (2014) 3198. arXiv:1409.3332, doi:10.1140/epjc/s10052-014-3198-3.

[202] X.-Y. Wang, X.-R. Chen, Production of the superheavy baryon $\Lambda_{c \bar{c}}^{*}(4209)$ in kaon-induced reaction, Eur. Phys. J. A51 (7) (2015) 85. arXiv:1504.01075, doi:10.1140/epja/i2015-15085-0.

[203] T. Uchino, W.-H. Liang, E. Oset, Baryon states with hidden charm in the extended local hidden gauge approach, Eur. Phys. J. A52 (3) (2016) 43. arXiv:1504.05726, doi:10.1140/epja/ i2016-16043-0.

[204] E. J. Garzon, J.-J. Xie, Effects of a $N_{c \bar{c}}^{*}$ resonance with hidden charm in the $\pi^{-} p \rightarrow D^{-} \Sigma_{c}^{+}$reaction near threshold, Phys. Rev. C92 (3) (2015) 035201. arXiv:1506.06834, doi:10.1103/PhysRevC. 92.035201.

[205] J. Hofmann, M. F. M. Lutz, Coupled-channel study of crypto-exotic baryons with charm, Nucl. Phys. A763 (2005) 90-139. arXiv:hep-ph/0507071, doi:10.1016/j.nuclphysa.2005.08.022.

[206] R. Chen, X. Liu, X.-Q. Li, S.-L. Zhu, Identifying exotic hidden-charm pentaquarks, Phys. Rev. Lett. 115 (13) (2015) 132002. arXiv:1507.03704, doi:10.1103/PhysRevLett.115.132002.

[207] H.-X. Chen, W. Chen, X. Liu, T. G. Steele, S.-L. Zhu, Towards exotic hidden-charm pentaquarks in QCD, Phys. Rev. Lett. 115 (17) (2015) 172001. arXiv:1507.03717, doi:10.1103/ PhysRevLett.115.172001.

[208] V. Kopeliovich, I. Potashnikova, Simple estimates of the masses of pentaquarks with hidden beauty or strangeness, Phys. Rev. D93 (2016) 074012. arXiv:1510.05958, doi:10.1103/PhysRevD.93. 074012.

[209] R. Chen, X. Liu, S.-L. Zhu, Hidden-charm molecular pentaquarks and their charm-strange partners, Nucl. Phys. A954 (2016) 406-421. arXiv:1601.03233, doi:10.1016/j.nuclphysa.2016. 04.012.

[210] L. Maiani, A. D. Polosa, V. Riquer, The New Pentaquarks in the Diquark Model, Phys. Lett. B749 (2015) 289-291. arXiv:1507.04980, doi:10.1016/j.physletb.2015.08.008.

[211] V. V. Anisovich, M. A. Matveev, J. Nyiri, A. V. Sarantsev, A. N. Semenova, Pentaquarks and resonances in the $p J / \psi$ spectrum, arXiv:1507.07652.

[212] A. Ali, I. Ahmed, M. J. Aslam, A. Rehman, Heavy quark symmetry and weak decays of the b-baryons in pentaquarks with a $c \bar{c}$ component, Phys. Rev. D94 (5) (2016) 054001. arXiv: 1607.00987, doi:10.1103/PhysRevD.94.054001.

[213] V. V. Anisovich, M. A. Matveev, A. V. Sarantsev, A. N. Semenova, Pentaquarks and strange tetraquark mesons, Mod. Phys. Lett. A30 (38) (2015) 1550212. arXiv:1509.03028, doi:10. 1142/S0217732315502120.

[214] V. V. Anisovich, M. A. Matveev, J. Nyiri, A. V. Sarantsev, A. N. Semenova, Non-strange and strange pentaquarks with hidden charm, Int. J. Mod. Phys. A30 (2015) 1550190. arXiv:1509. 04898, doi:10.1142/S0217751X15501900. 
[215] V. V. Anisovich, M. A. Matveev, J. Nyiri, A. N. Semenova, Narrow pentaquarks as diquarkCdiquarkCantiquark systems, Mod. Phys. Lett. A32 (29) (2017) 1750154. arXiv:1706.01336, doi:10.1142/S0217732317501541.

[216] V. V. Anisovich, M. A. Matveev, J. Nyiri, A. V. Sarantsev, A. N. Semenova, Diquark-diquarkantiquark model for pentaquarks with hidden charm: current status and problemsarXiv:1711. 10736.

[217] A. Ali, I. Ahmed, M. J. Aslam, A. Rehman, Mass spectrum of spin-1/2 pentaquarks with a $c \bar{c}$ component and their anticipated discovery modes in b-baryon decaysarXiv:1704.05419.

[218] Y. Irie, M. Oka, S. Yasui, Flavor-singlet hidden charm pentaquark, Phys. Rev. D97 (3) (2018) 034006. arXiv:1707.04544, doi:10.1103/PhysRevD.97.034006.

[219] F. Buccella, b decays: a factory for hidden charm multiquark states, Phys. Rev. D98 (11) (2018) 114011. arXiv:1801.03723, doi:10.1103/PhysRevD.98.114011.

[220] E. Santopinto, A. Giachino, Compact pentaquark structures, Phys. Rev. D96 (1) (2017) 014014. arXiv:1604.03769, doi:10.1103/PhysRevD.96.014014.

[221] F. Gursey, L. A. Radicati, Spin and unitary spin independence of strong interactions, Phys. Rev. Lett. 13 (1964) 173-175. doi:10.1103/PhysRevLett.13.173.

[222] E. Ortiz-Pacheco, R. Bijker, C. Fernúndez-Ramírez, Hidden charm pentaquarks: mass spectrum, magnetic moments, and photocouplingsarXiv:1808.10512.

[223] W. Park, S. Cho, S. H. Lee, Where is the stable PentaquarkarXiv:1811.10911.

[224] K.-W. Wei, B. Chen, X.-H. Guo, Masses of doubly and triply charmed baryons, Phys. Rev. D92 (7) (2015) 076008. arXiv:1503.05184, doi:10.1103/PhysRevD.92.076008.

[225] K.-W. Wei, B. Chen, N. Liu, Q.-Q. Wang, X.-H. Guo, Spectroscopy of singly, doubly, and triply bottom baryons, Phys. Rev. D95 (11) (2017) 116005. arXiv:1609.02512, doi:10.1103/ PhysRevD.95.116005.

[226] R. K. Bhaduri, L. E. Cohler, Y. Nogami, A Unified Potential for Mesons and Baryons, Nuovo Cim. A65 (1981) 376-390. doi :10.1007/BF02827441.

[227] B. Silvestre-Brac, Spectrum and static properties of heavy baryons, Few Body Syst. 20 (1996) 1-25. doi:10.1007/s006010050028.

[228] Y. S. Kalashnikova, A. V. Nefediev, X(3872) in the molecular modelarXiv:1811.01324, doi: 10.3367/UFNe.2018.08.038411.

[229] S. Weinberg, Evidence That the Deuteron Is Not an Elementary Particle, Phys. Rev. 137 (1965) B672-B678. doi:10.1103/PhysRev.137.B672.

[230] W.-J. Deng, H. Liu, L.-C. Gui, X.-H. Zhong, Charmonium spectrum and their electromagnetic transitions with higher multipole contributions, Phys. Rev. D95 (3) (2017) 034026. arXiv:1608. 00287, doi:10.1103/PhysRevD.95.034026.

[231] N. Achasov, Physics of the charmonium-like state X(3872), EPJ Web Conf. 125 (2016) 04002. doi:10.1051/epjconf/201612504002. 
[232] L.-C. Gui, L.-S. Lu, Q.-F. L, X.-H. Zhong, Q. Zhao, Strong decays of higher charmonium states into open-charm meson pairs, Phys. Rev. D98 (1) (2018) 016010. arXiv:1801.08791, doi:10. 1103/PhysRevD.98.016010.

[233] P. G. Ortega, J. Segovia, D. R. Entem, F. Fernndez, Canonical description of the new LHCb resonances, Phys. Rev. D94 (11) (2016) 114018. arXiv:1608.01325, doi:10.1103/PhysRevD. 94.114018.

[234] J. Vijande, F. Fernandez, A. Valcarce, Constituent quark model study of the meson spectra, J. Phys. G31 (2005) 481. arXiv:hep-ph/0411299, doi:10.1088/0954-3899/31/5/017.

[235] J. Segovia, A. M. Yasser, D. R. Entem, F. Fernandez, $J^{P C}=1^{--}$hidden charm resonances, Phys. Rev. D78 (2008) 114033. doi:10.1103/PhysRevD.78.114033.

[236] D. Molina, M. De Sanctis, C. Fernandez-Ramirez, Charmonium spectrum with a Dirac potential model in the momentum space, Phys. Rev. D95 (9) (2017) 094021. arXiv:1703.08097, doi: 10.1103/PhysRevD.95.094021.

[237] T. Bhavsar, M. Shah, P. C. Vinodkumar, Status of quarkonia-like negative and positive parity states in a relativistic confinement scheme, Eur. Phys. J. C78 (3) (2018) 227. arXiv:1803.07249, doi:10.1140/epjc/s10052-018-5694-3.

[238] V. Kher, A. K. Rai, Spectroscopy and decay properties of charmonium, Chin. Phys. C42 (8) (2018) 083101. arXiv: 1805.02534, doi:10.1088/1674-1137/42/8/083101.

[239] H.-F. Fu, L. Jiang, Coupled-Channel-Induced $S-D$ mixing of Charmonia and Possible Assignments for $Y(4260)$ and $Y(4360)$ arXiv:1812.00179.

[240] R. Oncala, J. Soto, Heavy Quarkonium Hybrids: Spectrum, Decay and Mixing, Phys. Rev. D96 (1) (2017) 014004. arXiv:1702.03900, doi:10.1103/PhysRevD.96.014004.

[241] K. F. Chen, et al., Observation of an enhancement in $e^{+} e^{-} \rightarrow \Upsilon(1 S) \pi^{+} \pi^{-}, \Upsilon(2 S) \pi^{+} \pi^{-}$, and $\Upsilon(3 S) \pi^{+} \pi^{-}$production around $\sqrt{s}=10.89 \mathrm{GeV}$ at Belle, Phys. Rev. D82 (2010) 091106. arXiv: 0810.3829, doi:10.1103/PhysRevD.82.091106.

[242] M. Berwein, N. Brambilla, J. Tarrús Castellà, A. Vairo, Quarkonium Hybrids with Nonrelativistic Effective Field Theories, Phys. Rev. D92 (11) (2015) 114019. arXiv:1510.04299, doi:10.1103/ PhysRevD.92.114019.

[243] T. Miyamoto, S. Yasui, Hyperspherical-coordinate approach to the spectra and decay widths of hybrid quarkoniaA hyperspherical-coordinate approach to the spectra and decay widths of hybrid quarkonia, Phys. Rev. D98 (9) (2018) 094027. arXiv:1806.07970, doi:10.1103/PhysRevD.98. 094027.

[244] R. Bruschini, P. González, A plausible explanation of $\Upsilon(10860)$, Phys. Lett. B791 (2019) 409-413. arXiv:1811.08236, doi:10.1016/j.physletb.2019.03.017.

[245] M. Ablikim, et al., Observation of the decay $X(3872) \rightarrow \pi^{0} \chi_{c 1}(1 P) \operatorname{arXiv}: 1901.03992$.

[246] S. Dubynskiy, M. B. Voloshin, Pionic transitions from X(3872) to $\chi_{c J}$, Phys. Rev. D77 (2008) 014013. arXiv:0709.4474, doi:10.1103/PhysRevD.77.014013. 
[247] B. Aubert, et al., Evidence for $X(3872) \rightarrow \psi(2 S) \gamma$ in $B^{ \pm} \rightarrow X(3872) K^{ \pm}$decays, and a study of $B \rightarrow c \bar{c} \gamma K$, Phys. Rev. Lett. 102 (2009) 132001. arXiv:0809.0042, doi:10.1103/PhysRevLett. 102.132001.

[248] V. Bhardwaj, et al., Observation of $X(3872) \rightarrow J / \psi \gamma$ and search for $X(3872) \rightarrow \psi^{\prime} \gamma$ in $B$ decays, Phys. Rev. Lett. 107 (2011) 091803. arXiv:1105.0177, doi:10.1103/PhysRevLett.107.091803.

[249] E. Cincioglu, J. Nieves, A. Ozpineci, A. U. Yilmazer, Quarkonium Contribution to Meson Molecules, Eur. Phys. J. C76 (10) (2016) 576. arXiv:1606.03239, doi:10.1140/epjc/ s10052-016-4413-1.

[250] Z.-Y. Zhou, Z. Xiao, Understanding X(3862), X(3872), and X(3930) in a Friedrichs-model-like scheme, Phys. Rev. D96 (5) (2017) 054031, [Erratum: Phys. Rev.D96,no.9,099905(2017)]. arXiv: 1704.04438, doi:10.1103/PhysRevD.96.099905,10.1103/PhysRevD.96.054031.

[251] Z.-Y. Zhou, Z. Xiao, Comprehending Isospin breaking effects of $X(3872)$ in a Friedrichs-modellike scheme, Phys. Rev. D97 (3) (2018) 034011. arXiv:1711.01930, doi:10.1103/PhysRevD.97. 034011.

[252] Z.-Y. Zhou, D.-Y. Chen, Z. Xiao, Does the bottomonium counterpart of X(3872) exist?, Phys. Rev. D99 (3) (2019) 034005. arXiv:1810.03452, doi:10.1103/PhysRevD.99.034005.

[253] S. Chatrchyan, et al., Search for a new bottomonium state decaying to $\Upsilon(1 S) \pi^{+} \pi^{-}$in pp collisions at $\sqrt{s}=8 \mathrm{TeV}$, Phys. Lett. B727 (2013) 57-76. arXiv:1309.0250, doi:10.1016/j.physletb. 2013.10 .016 .

[254] X. H. He, et al., Observation of $e^{+} e^{-} \rightarrow \pi^{+} \pi^{-} \pi^{0} \chi_{b J}$ and Search for $X_{b} \rightarrow \omega \Upsilon(1 S)$ at $\sqrt{s}=10.867$ GeV, Phys. Rev. Lett. 113 (14) (2014) 142001. arXiv:1408.0504, doi:10.1103/PhysRevLett. 113.142001.

[255] J. Ferretti, E. Santopinto, Threshold corrections of $\chi_{c}(2 \mathrm{P})$ and $\chi_{b}(3 \mathrm{P})$ states and J $/ \psi \rho$ and $\mathrm{J} / \psi \omega$ transitions of the $\chi$ (3872) in a coupled-channel model, Phys. Lett. B789 (2019) 550-555. arXiv:1806.02489, doi:10.1016/j.physletb.2018.12.052.

[256] P. G. Ortega, D. R. Entem, F. Fernndez, Unquenching the quark model in a non-perturbative schemearXiv: 1901.02484.

[257] Y. Lu, M. N. Anwar, B.-S. Zou, Coupled-Channel Effects for the Bottomonium with Realistic Wave Functions, Phys. Rev. D94 (3) (2016) 034021. arXiv:1606.06927, doi:10.1103/PhysRevD. 94.034021.

[258] E. Cincioglu, A. Ozpineci, Radiative decay of the $X(3872)$ as a mixed molecule-charmonium state in effective field theoryarXiv:1901.03138.

[259] F. Giacosa, M. Piotrowska, S. Coito, X(3872) as virtual companion pole of the charm-anticharm state $\chi_{c 1}(2 P)$ arXiv: 1903.06926.

[260] W. Qin, S.-R. Xue, Q. Zhao, Production of $Y(4260)$ as a hadronic molecule state of $\bar{D} D_{1}+$ c.c. in $e^{+} e^{-}$annihilations, Phys. Rev. D94 (5) (2016) 054035. arXiv:1605.02407, doi:10.1103/ PhysRevD.94.054035.

[261] Y. Lu, M. N. Anwar, B.-S. Zou, X(4260) Revisited: A Coupled Channel Perspective, Phys. Rev. D96 (11) (2017) 114022. arXiv:1705.00449, doi:10.1103/PhysRevD.96.114022. 
[262] H. X. Zhang, M. Zhang, Z. Y. Zhang, Study of Qq anti-Q anti-q-prime states in chiral SU(3) quark model, Chin. Phys. Lett. 24 (2007) 2533-2536. arXiv:0705.2470, doi:10.1088/0256-307X/24/ 9/019.

[263] J. Vijande, E. Weissman, N. Barnea, A. Valcarce, Do $c \bar{c} n \bar{n}$ bound states exist?, Phys. Rev. D76 (2007) 094022. arXiv:0708.3285, doi:10.1103/PhysRevD.76.094022.

[264] S. Coito, Radially excited axial mesons and the enigmatic $Z_{c}$ and $Z_{b}$ in a coupled-channel model, Phys. Rev. D94 (1) (2016) 014016. arXiv:1602.07821, doi:10.1103/PhysRevD.94.014016.

[265] S. Patel, P. C. Vinodkumar, Tetraquark states in the bottom sector and the status of the $Y_{b}$ (10890) state, Eur. Phys. J. C76 (7) (2016) 356. arXiv:1606.01047, doi:10.1140/epjc/ s10052-016-4186-6.

[266] Q.-F. L, Y.-B. Dong, X(4140), X(4274), X(4500), and X(4700) in the relativized quark model, Phys. Rev. D94 (7) (2016) 074007. arXiv:1607.05570, doi:10.1103/PhysRevD.94.074007.

[267] H.-X. Chen, E.-L. Cui, W. Chen, X. Liu, S.-L. Zhu, Understanding the internal structures of the $X(4140), X(4274), X(4500)$ and $X(4700)$, Eur. Phys. J. C77 (3) (2017) 160. arXiv:1606.03179, doi : 10.1140/epjc/s10052-017-4737-5.

[268] Z.-G. Wang, Scalar tetraquark state candidates: $X(3915), X(4500)$ and $X(4700)$, Eur. Phys. J. C77 (2) (2017) 78. arXiv:1606.05872, doi:10.1140/epjc/s10052-017-4640-0.

[269] T. Barnes, S. Godfrey, E. S. Swanson, Higher charmonia, Phys. Rev. D72 (2005) 054026. arXiv: hep-ph/0505002, doi:10.1103/PhysRevD.72.054026.

[270] X.-H. Liu, How to understand the underlying structures of $X(4140), X(4274), X(4500)$ and $X(4700)$, Phys. Lett. B766 (2017) 117-124. arXiv:1607.01385, doi:10.1016/j.physletb. 2017.01 .008$.

[271] Y.-C. Yang, Z.-Y. Tan, J. Ping, H.-S. Zong, Possible $D^{(*)} \bar{D}^{(*)}$ and $B^{(*)} \bar{B}^{(*)}$ molecular states in the extended constituent quark models, Eur. Phys. J. C77 (9) (2017) 575. arXiv:1703.09718, doi:10.1140/epjc/s10052-017-5137-6.

[272] Y.-C. Yang, Z.-Y. Tan, H.-S. Zong, J. Ping, Dynamical study of $S$-wave $\bar{Q} Q \bar{q} q$ system, Few Body Syst. 60 (1) (2019) 9. arXiv:1712.09285, doi:10.1007/s00601-018-1477-5.

[273] M. N. Anwar, J. Ferretti, E. Santopinto, Spectroscopy of the hidden-charm $[q c][\bar{q} \bar{c}]$ and $[s c][\bar{s} \bar{c}]$ tetraquarks in the relativized diquark model, Phys. Rev. D98 (9) (2018) 094015. arXiv:1805. 06276, doi:10.1103/PhysRevD.98.094015.

[274] J. F. Giron, R. F. Lebed, C. T. Peterson, The Dynamical Diquark Model: First Numerical ResultsarXiv: 1903.04551.

[275] Y. Yang, J. Ping, Investigation of $\operatorname{cs} \bar{c} \bar{s}$ tetraquark in the chiral quark modelarXiv:1903.08505.

[276] D. Janc, M. Rosina, The $T_{c c}=D D^{*}$ molecular state, Few Body Syst. 35 (2004) 175-196. arXiv: hep-ph/0405208, doi:10.1007/s00601-004-0068-9.

[277] M. Zhang, H. X. Zhang, Z. Y. Zhang, $Q Q \bar{q} \bar{q}$ four-quark bound states in chiral SU(3) quark model, Commun. Theor. Phys. 50 (2008) 437-440. arXiv:0711.1029, doi:10.1088/0253-6102/50/2/ 31. 
[278] Y. Yang, C. Deng, J. Ping, T. Goldman, S-wave $Q Q \bar{q} \bar{q}$ state in the constituent quark model, Phys. Rev. D80 (2009) 114023. doi:10.1103/PhysRevD.80.114023.

[279] J. Vijande, A. Valcarce, N. Barnea, Exotic meson-meson molecules and compact four-quark states, Phys. Rev. D79 (2009) 074010. arXiv:0903.2949, doi:10.1103/PhysRevD.79.074010.

[280] J. Vijande, F. Fernandez, A. Valcarce, B. Silvestre-Brac, Tetraquarks in a chiral constituent quark model, Eur. Phys. J. A19 (2004) 383. arXiv:hep-ph/0310007, doi:10.1140/epja/ i2003-10128-9.

[281] J. Vijande, A. Valcarce, K. Tsushima, Dynamical study of bf QQ - anti-u anti-d mesons, Phys. Rev. D74 (2006) 054018. arXiv:hep-ph/0608316, doi:10.1103/PhysRevD .74.054018.

[282] J. Vijande, E. Weissman, A. Valcarce, N. Barnea, Are there compact heavy four-quark bound states?, Phys. Rev. D76 (2007) 094027. arXiv:0710.2516, doi:10.1103/PhysRevD.76.094027.

[283] T. F. Carams, J. Vijande, A. Valcarce, Exotic bc $\bar{q} \bar{q}$ four-quark states, Phys. Rev. D99 (1) (2019) 014006. arXiv:1812.08991, doi:10.1103/PhysRevD.99.014006.

[284] G. Q. Feng, X. H. Guo, B. S. Zou, $Q Q^{\prime} \bar{u} \bar{d}$ bound state in the Bethe-Salpeter equation approacharXiv: 1309.7813.

[285] W. Park, S. H. Lee, Color spin wave functions of heavy tetraquark states, Nucl. Phys. A925 (2014) 161-184. arXiv:1311.5330, doi:10.1016/j.nuclphysa.2014.02.008.

[286] W. Park, S. Noh, S. H. Lee, Masses of the doubly heavy tetraquarks in a constituent quark model, Nucl. Phys. A983 (2019) 1-19. arXiv:1809.05257, doi:10.1016/j.nuclphysa.2018.12.019.

[287] A. Czarnecki, B. Leng, M. B. Voloshin, Stability of tetrons, Phys. Lett. B778 (2018) 233-238. arXiv:1708.04594, doi:10.1016/j.physletb.2018.01.034.

[288] J.-M. Richard, A. Valcarce, J. Vijande, Few-body quark dynamics for doubly heavy baryons and tetraquarks, Phys. Rev. C97 (3) (2018) 035211. arXiv:1803.06155, doi:10.1103/PhysRevC. 97.035211.

[289] R. J. Lloyd, J. P. Vary, All charm tetraquarks, Phys. Rev. D70 (2004) 014009. arXiv:hep-ph/ 0311179, doi:10.1103/PhysRevD.70.014009.

[290] M. N. Anwar, J. Ferretti, F.-K. Guo, E. Santopinto, B.-S. Zou, Spectroscopy and decays of the fully-heavy tetraquarks, Eur. Phys. J. C78 (8) (2018) 647. arXiv:1710.02540, doi:10.1140/ epjc/s10052-018-6073-9.

[291] Y. Bai, S. Lu, J. Osborne, Beauty-full TetraquarksarXiv:1612.00012.

[292] N. Barnea, J. Vijande, A. Valcarce, Four-quark spectroscopy within the hyperspherical formalism, Phys. Rev. D73 (2006) 054004. arXiv:hep-ph/0604010, doi:10.1103/PhysRevD.73.054004.

[293] V. R. Debastiani, F. S. Navarra, A non-relativistic model for the $[c c][\bar{c} \bar{c}]$ tetraquark, Chin. Phys. C43 (1) (2019) 013105. arXiv:1706.07553, doi:10.1088/1674-1137/43/1/013105.

[294] J.-M. Richard, A. Valcarce, J. Vijande, String dynamics and metastability of all-heavy tetraquarks, Phys. Rev. D95 (5) (2017) 054019. arXiv:1703.00783, doi:10.1103/PhysRevD. 95.054019.

[295] X. Chen, Analysis of beauty-full $b b \bar{b} \bar{b}$ statearXiv: 1902.00008. 
[296] H. Huang, C. Deng, J. Ping, F. Wang, Possible pentaquarks with heavy quarks, Eur. Phys. J. C76 (11) (2016) 624. arXiv:1510.04648, doi:10.1140/epjc/s10052-016-4476-z.

[297] H. Huang, X. Zhu, J. Ping, $P_{c}$-like pentaquarks in hidden strange sector, Phys. Rev. D97 (9) (2018) 094019. arXiv:1803.05267, doi:10.1103/PhysRevD.97.094019.

[298] G. Yang, J. Ping, The structure of pentaquarks $P_{c}^{+}$in the chiral quark model, Phys. Rev. D95 (1) (2017) 014010. arXiv:1511.09053, doi:10.1103/PhysRevD.95.014010.

[299] G. Yang, J. Ping, J. Segovia, Hidden-bottom pentaquarks, Phys. Rev. D99 (1) (2019) 014035. arXiv:1809.06193, doi:10.1103/PhysRevD.99.014035.

[300] S. M. Gerasyuta, V. I. Kochkin, Relativistic five-quark equations and the LHCb pentaquarksarXiv:1512.04040.

[301] P. G. Ortega, D. R. Entem, F. Fernndez, LHCb pentaquarks in constituent quark models, Phys. Lett. B764 (2017) 207-211. arXiv:1606.06148, doi:10.1016/j.physletb.2016.11.008.

[302] S. Takeuchi, M. Takizawa, The hidden charm pentaquarks are the hidden color-octet uud baryons?, Phys. Lett. B764 (2017) 254-259. arXiv:1608.05475, doi:10.1016/j.physletb. 2016.11 .034$.

[303] W. Park, A. Park, S. Cho, S. H. Lee, $P_{c}(4380)$ in a constituent quark model, Phys. Rev. D95 (5) (2017) 054027. arXiv:1702.00381, doi:10.1103/PhysRevD.95.054027.

[304] F. Stancu, Stability of pentaquarks with a two- plus three-body chromoelectric interaction, Phys. Rev. D96 (1) (2017) 014007. arXiv:1705.02490, doi:10.1103/PhysRevD.96.014007.

[305] J. M. Richard, A. Valcarce, J. Vijande, Stable heavy pentaquarks in constituent models, Phys. Lett. B774 (2017) 710-714. arXiv:1710.08239, doi:10.1016/j.physletb.2017.10.036.

[306] E. Hiyama, A. Hosaka, M. Oka, J.-M. Richard, Quark model estimate of hidden-charm pentaquark resonances, Phys. Rev. C98 (4) (2018) 045208. arXiv:1803.11369, doi:10.1103/PhysRevC.98. 045208.

[307] F. Stancu, Spectrum of the uudcc hidden charm pentaquark with an SU(4) flavor-spin hyperfine interactionarXiv: 1902.07101.

[308] F. Giannuzzi, Heavy pentaquark spectroscopy in the diquark modelarXiv: 1903.04430.

[309] G.-J. Wang, R. Chen, L. Ma, X. Liu, S.-L. Zhu, Magnetic moments of the hidden-charm pentaquark states, Phys. Rev. D94 (9) (2016) 094018. arXiv:1605.01337, doi:10.1103/PhysRevD. 94.094018.

[310] G.-J. Wang, Z.-W. Liu, S.-L. Zhu, Axial charges of the hidden-charm pentaquark states, Phys. Rev. C94 (6) (2016) 065202. arXiv:1608.07824, doi:10.1103/PhysRevC.94.065202.

[311] N. Isgur, J. E. Paton, A Flux Tube Model for Hadrons, Phys. Lett. 124B (1983) 247-251. doi: 10.1016/0370-2693(83)91445-4.

[312] N. Isgur, J. E. Paton, A Flux Tube Model for Hadrons in QCD, Phys. Rev. D31 (1985) 2910. doi:10.1103/PhysRevD.31.2910.

[313] J. B. Kogut, L. Susskind, Hamiltonian Formulation of Wilson's Lattice Gauge Theories, Phys. Rev. D11 (1975) 395-408. doi:10.1103/PhysRevD.11.395. 
[314] P. Gnadig, P. Hasenfratz, J. Kuti, A. S. Szalay, The Quark Bag Model with Surface Tension, Phys. Lett. 64B (1976) 62-66. doi:10.1016/0370-2693(76)90358-0.

[315] T. Barnes, F. E. Close, E. S. Swanson, Hybrid and conventional mesons in the flux tube model: Numerical studies and their phenomenological implications, Phys. Rev. D52 (1995) 5242-5256. arXiv:hep-ph/9501405, doi:10.1103/PhysRevD.52.5242.

[316] J. Merlin, J. E. Paton, Spin Interactions in the Flux Tube Model and Hybrid Meson Masses, Phys. Rev. D35 (1987) 1668. doi:10.1103/PhysRevD.35.1668.

[317] P. R. Page, E. S. Swanson, A. P. Szczepaniak, Hybrid meson decay phenomenology, Phys. Rev. D59 (1999) 034016. arXiv:hep-ph/9808346, doi:10.1103/PhysRevD.59.034016.

[318] F. Wang, C. W. Wong, MULTI-QUARK STRINGS, Nuovo Cim. A86 (1985) 283, [Erratum: Nuovo Cim.A90,324(1985)]. doi:10.1007/BF02812694.

[319] J.-l. Ping, C.-r. Deng, F. Wang, Quantum chromodynamics quark benzene, Phys. Lett. B659 (2008) 607-611. arXiv:hep-ph/0610390, doi:10.1016/j.physletb.2007.11.051.

[320] C. Deng, J. Ping, F. Wang, Interpreting $Z_{c}(3900)$ and $Z_{c}(4025) / Z_{c}(4020)$ as charged tetraquark states, Phys. Rev. D90 (5) (2014) 054009. arXiv:1402.0777, doi:10.1103/PhysRevD.90. 054009.

[321] C. Deng, J. Ping, H. Huang, F. Wang, Systematic study of $Z_{c}^{+}$family from a multiquark color fluxtube model, Phys. Rev. D92 (3) (2015) 034027. arXiv:1507.06408, doi:10.1103/PhysRevD.92. 034027.

[322] P. Zhou, C.-R. Deng, J.-L. Ping, Identification of $Y(4008), Y(4140), Y(4260)$, and $Y(4360)$ as Tetraquark States, Chin. Phys. Lett. 32 (10) (2015) 101201. doi:10.1088/0256-307X/32/10/ 101201.

[323] C. Deng, J. Ping, H. Huang, F. Wang, Heavy pentaquark states and a novel color structure, Phys. Rev. D95 (1) (2017) 014031. arXiv:1608.03940, doi:10.1103/PhysRevD.95.014031.

[324] C. Deng, H. Chen, J. Ping, Systematical investigation on the stability of doubly heavy tetraquark statesarXiv: 1811.06462.

[325] P. Bicudo, M. Cardoso, Tetraquark bound states and resonances in the unitary and microscopic triple string flip-flop quark model, the light-light-antiheavy-antiheavy $q q \bar{Q} \bar{Q}$ case studyarXiv: 1509.04943.

[326] M.-T. Li, Y.-B. Dong, Z.-Y. Zhang, A study of meson-meson potential in the chiral quark model, Chin. Phys. C35 (2011) 622-628. arXiv:1010.2283, doi:10.1088/1674-1137/35/7/005.

[327] J. Ferretti, E. Santopinto, M. Naeem Anwar, M. A. Bedolla, The baryo-quarkonium picture for hidden-charm and bottom pentaquarks and LHCb $P_{\mathrm{c}}(4380)$ and $P_{\mathrm{c}}(4450)$ states, Phys. Lett. B789 (2019) 562-567. arXiv:1807.01207, doi:10.1016/j.physletb.2018.09.047.

[328] S. Weinberg, Nuclear forces from chiral Lagrangians, Phys. Lett. B251 (1990) 288-292. doi: 10.1016/0370-2693(90)90938-3.

[329] S. Weinberg, Effective chiral Lagrangians for nucleon-pion interactions and nuclear forces, Nucl. Phys. B363 (1991) 3-18. doi:10.1016/0550-3213(91) 90231-L. 
[330] D. P. Rathaud, A. K. Rai, Dimesonic states with the heavy-light flavour mesons, Eur. Phys. J. Plus 132 (8) (2017) 370. arXiv:1608.03781, doi:10.1140/epjp/i2017-11641-3.

[331] Y. Liu, I. Zahed, Heavy Exotic Molecules with Charm and Bottom, Phys. Lett. B762 (2016) 362-370. arXiv:1608.06535, doi:10.1016/j.physletb.2016.09.045.

[332] M.-Z. Liu, D.-J. Jia, D.-Y. Chen, Possible hadronic molecular states composed of $S$-wave heavylight mesons, Chin. Phys. C41 (5) (2017) 053105. arXiv:1702.04440, doi:10.1088/1674-1137/ 41/5/053105.

[333] X. Chen, X. Lu, R. Shi, X. Guo, Mass of $Y(4140)$ in Bethe-Salpeter equation for quarksarXiv: 1512.06483.

[334] Z.-Y. Wang, J.-J. Qi, X.-H. Guo, C. Wang, X(3872) as a molecular D $\bar{D}^{*}$ state in the BetheSalpeter equation approach, Phys. Rev. D97 (1) (2018) 016015. arXiv:1710.07424, doi:10. 1103/PhysRevD.97.016015.

[335] J. He, Understanding spin parity of $P_{c}(4450)$ and $Y(4274)$ in a hadronic molecular state picture, Phys. Rev. D95 (7) (2017) 074004. arXiv:1607.03223, doi:10.1103/PhysRevD.95.074004.

[336] J. He, D.-Y. Chen, Interpretation of $Y(4390)$ as an isoscalar partner of $Z(4430)$ from $D^{*}(2010) \bar{D}_{1}(2420)$ interaction, Eur. Phys. J. C77 (6) (2017) 398. arXiv:1704.08776, doi: 10.1140/epjc/s10052-017-4973-8.

[337] D.-Y. Chen, C.-J. Xiao, J. He, Hidden-charm decays of Y(4390) in a hadronic molecular scenario, Phys. Rev. D96 (5) (2017) 054017. doi:10.1103/PhysRevD.96.054017.

[338] S. Sakai, L. Roca, E. Oset, Charm-beauty meson bound states from $B\left(B^{*}\right) D\left(D^{*}\right)$ and $B\left(B^{*}\right) \bar{D}\left(\bar{D}^{*}\right)$ interaction, Phys. Rev. D96 (5) (2017) 054023. arXiv:1704.02196, doi:10.1103/ PhysRevD.96.054023.

[339] B.-X. Sun, D.-M. Wan, S.-Y. Zhao, The $D \bar{D}^{*}$ interaction with isospin zero in an extended hidden gauge symmetry approach, Chin. Phys. C42 (5) (2018) 053105. arXiv:1709.07263, doi:10. 1088/1674-1137/42/5/053105.

[340] P. G. Ortega, J. Segovia, D. R. Entem, F. Fernández, The $Z_{c}$ structures in a coupledchannels model, Eur. Phys. J. C79 (1) (2019) 78. arXiv:1808.00914, doi:10.1140/epjc/ s10052-019-6552-7.

[341] J. Y. Panteleeva, I. A. Perevalova, M. V. Polyakov, P. Schweitzer, On tetraquarks with hidden charm and strangeness as phi-psi(2S) hadrocharmoniumarXiv:1802.09029.

[342] J. Ferretti, $\eta_{\mathrm{c}^{-}}$and $J / \psi$-isoscalar meson bound states in the hadro-charmonium picture, Phys. Lett. B782 (2018) 702-706. arXiv:1805.04717, doi:10.1016/j.physletb.2018.06.032.

[343] R. Molina, E. Oset, The $Y(3940), Z(3930)$ and the $X(4160)$ as dynamically generated resonances from the vector-vector interaction, Phys. Rev. D80 (2009) 114013. arXiv:0907.3043, doi:10. 1103/PhysRevD.80.114013.

[344] M. B. Voloshin, $Z_{c}(4100)$ and $Z_{c}(4200)$ as hadrocharmonium, Phys. Rev. D98 (9) (2018) 094028. arXiv:1810.08146, doi:10.1103/PhysRevD.98.094028.

[345] M. B. Voloshin, Strange hadrocharmoniumarXiv: 1901.01936. 
[346] R. Gao, Z.-H. Guo, X.-W. Kang, J. A. Oller, Effective-range-expansion study of near threshold heavy-flavor resonancesarXiv: 1812.07323.

[347] X.-W. Kang, Z.-H. Guo, J. A. Oller, General considerations on the nature of $Z_{b}(10610)$ and $Z_{b}(10650)$ from their pole positions, Phys. Rev. D94 (1) (2016) 014012. arXiv:1603.05546, doi:10.1103/PhysRevD.94.014012.

[348] J. M. Dias, F. Aceti, E. Oset, Study of $B \bar{B}^{*}$ and $B^{*} \bar{B}^{*}$ interactions in $I=1$ and relationship to the $Z_{b}(10610), Z_{b}(10650)$ states, Phys. Rev. D91 (7) (2015) 076001. arXiv:1410.1785, doi: 10.1103/PhysRevD.91.076001.

[349] R. Molina, T. Branz, E. Oset, A new interpretation for the $D_{s 2}^{*}(2573)$ and the prediction of novel exotic charmed mesons, Phys. Rev. D82 (2010) 014010. arXiv:1005.0335, doi:10.1103/ PhysRevD . 82.014010.

[350] T. F. Carames, A. Valcarce, J. Vijande, Doubly charmed exotic mesons: A gift of nature?, Phys. Lett. B699 (2011) 291-295. doi:10.1016/j.physletb.2011.04.023.

[351] A. Valcarce, H. Garcilazo, F. Fernandez, P. Gonzalez, Quark-model study of few-baryon systems, Rept. Prog. Phys. 68 (2005) 965-1042. arXiv:hep-ph/0502173, doi:10.1088/0034-4885/68/ 5/R01.

[352] S. Ohkoda, Y. Yamaguchi, S. Yasui, K. Sudoh, A. Hosaka, Exotic mesons with double charm and bottom flavor, Phys. Rev. D86 (2012) 034019. arXiv:1202.0760, doi:10.1103/PhysRevD.86. 034019.

[353] N. Li, Z.-F. Sun, X. Liu, S.-L. Zhu, Coupled-channel analysis of the possible $D^{(*)} D^{(*)}, \bar{B}^{(*)} \bar{B}^{(*)}$ and $D^{(*)} \bar{B}^{(*)}$ molecular states, Phys. Rev. D88 (11) (2013) 114008. arXiv:1211.5007, doi: 10.1103/PhysRevD.88.114008.

[354] Z.-F. Sun, X. Liu, M. Nielsen, S.-L. Zhu, Hadronic molecules with both open charm and bottom, Phys. Rev. D85 (2012) 094008. arXiv:1203.1090, doi:10.1103/PhysRevD.85.094008.

[355] P. Bicudo, M. Wagner, Lattice QCD signal for a bottom-bottom tetraquark, Phys. Rev. D87 (11) (2013) 114511. arXiv:1209.6274, doi:10.1103/PhysRevD.87.114511.

[356] P. Bicudo, K. Cichy, A. Peters, M. Wagner, BB interactions with static bottom quarks from Lattice QCD, Phys. Rev. D93 (3) (2016) 034501. arXiv:1510.03441, doi:10.1103/PhysRevD. 93.034501.

[357] P. Bicudo, K. Cichy, A. Peters, B. Wagenbach, M. Wagner, Evidence for the existence of $u d \bar{b} \bar{b}$ and the non-existence of $s s \bar{b} \bar{b}$ and $c c \bar{b} \bar{b}$ tetraquarks from lattice QCD, Phys. Rev. D92 (1) (2015) 014507. arXiv:1505.00613, doi:10.1103/PhysRevD.92.014507.

[358] P. Bicudo, M. Cardoso, A. Peters, M. Pflaumer, M. Wagner, ud $\bar{b} \bar{b}$ tetraquark resonances with lattice QCD potentials and the Born-Oppenheimer approximation, Phys. Rev. D96 (5) (2017) 054510. arXiv:1704.02383, doi:10.1103/PhysRevD.96.054510.

[359] M. Sanchez Sanchez, L.-S. Geng, J.-X. Lu, T. Hyodo, M. P. Valderrama, Exotic doubly charmed $D_{s 0}^{*}(2317) D$ and $D_{s 1}^{*}(2460) D^{*}$ molecules, Phys. Rev. D98 (5) (2018) 054001. arXiv:1707.03802, doi:10.1103/PhysRevD.98.054001. 
[360] H. Xu, B. Wang, Z.-W. Liu, X. Liu, DD* potentials in chiral perturbation theory and possible molecular states, Phys. Rev. D99 (1) (2019) 014027. arXiv:1708.06918, doi:10.1103/ PhysRevD.99.014027.

[361] B. Wang, Z.-W. Liu, X. Liu, $\bar{B}^{(*)} \bar{B}^{(*)}$ interactions in chiral effective field theory, Phys. Rev. D99 (3) (2019) 036007. arXiv:1812.04457, doi:10.1103/PhysRevD.99.036007.

[362] Y. Shimizu, D. Suenaga, M. Harada, Coupled channel analysis of molecule picture of $P_{c}(4380)$, Phys. Rev. D93 (11) (2016) 114003. arXiv:1603.02376, doi:10.1103/PhysRevD.93.114003.

[363] Y. Shimizu, M. Harada, Hidden Charm Pentaquark $P_{c}(4380)$ and Doubly Charmed Baryon $\Xi_{c c}^{*}(4380)$ as Hadronic Molecule States, Phys. Rev. D96 (9) (2017) 094012. arXiv:1708.04743, doi:10.1103/PhysRevD.96.094012.

[364] Y. Yamaguchi, E. Santopinto, Hidden-charm pentaquarks as a meson-baryon molecule with coupled channels for $\bar{D}^{(*)} \Lambda_{\mathrm{c}}$ and $\bar{D}^{(*)} \Sigma_{\mathrm{c}}^{(*)}$, Phys. Rev. D96 (1) (2017) 014018. arXiv:1606.08330, doi:10.1103/PhysRevD.96.014018.

[365] Y. Yamaguchi, A. Giachino, A. Hosaka, E. Santopinto, S. Takeuchi, M. Takizawa, Hidden-charm and bottom meson-baryon molecules coupled with five-quark states, Phys. Rev. D96 (11) (2017) 114031. arXiv:1709.00819, doi:10.1103/PhysRevD.96.114031.

[366] J. He, Nucleon resonances $N(1875)$ and $N(2100)$ as strange partners of LHCb pentaquarks, Phys. Rev. D95 (7) (2017) 074031. arXiv:1701.03738, doi:10.1103/PhysRevD.95.074031.

[367] R. Chen, J. He, X. Liu, Possible strange hidden-charm pentaquarks from $\Sigma_{c}^{(*)} \bar{D}_{s}^{*}$ and $\Xi_{c}^{\left({ }^{\prime}, *\right)} \bar{D}^{*}$ interactions, Chin. Phys. C41 (10) (2017) 103105. arXiv:1609.03235, doi:10.1088/1674-1137/ $41 / 10 / 103105$.

[368] L. Geng, J. Lu, M. P. Valderrama, Scale Invariance in Heavy Hadron Molecules, Phys. Rev. D97 (9) (2018) 094036. arXiv:1704.06123, doi:10.1103/PhysRevD.97.094036.

[369] R. Chen, A. Hosaka, X. Liu, Heavy molecules and one- $\sigma / \omega$-exchange model, Phys. Rev. D96 (11) (2017) 116012. arXiv:1707.08306, doi:10.1103/PhysRevD.96.116012.

[370] D. P. Rathaud, A. K. Rai, Interaction and Identification of the Meson-Baryon moleculesarXiv: 1808.05815.

[371] C.-W. Shen, D. Rönchen, U.-G. Meissner, B.-S. Zou, Exploratory study of possible resonances in heavy meson - heavy baryon coupled-channel interactions, Chin. Phys. C42 (2) (2018) 023106. arXiv:1710.03885, doi:10.1088/1674-1137/42/2/023106.

[372] H. Huang, J. Ping, Investigating the hidden-charm and hidden-bottom pentaquark resonances in scattering process, Phys. Rev. D99 (1) (2019) 014010. arXiv:1811.04260, doi:10.1103/ PhysRevD.99.014010.

[373] M. I. Eides, V. Yu. Petrov, M. V. Polyakov, Narrow Nucleon- $\psi(2 S)$ Bound State and LHCb Pentaquarks, Phys. Rev. D93 (5) (2016) 054039. arXiv:1512.00426, doi:10.1103/PhysRevD. 93.054039.

[374] I. A. Perevalova, M. V. Polyakov, P. Schweitzer, On LHCb pentaquarks as a baryon- $\psi(2 S)$ bound state: prediction of isospin- $\frac{3}{2}$ pentaquarks with hidden charm, Phys. Rev. D94 (5) (2016) 054024. arXiv:1607.07008, doi:10.1103/PhysRevD.94.054024. 
[375] M. I. Eides, V. Yu. Petrov, M. V. Polyakov, Pentaquarks with hidden charm as hadroquarkonia, Eur. Phys. J. C78 (1) (2018) 36. arXiv:1709.09523, doi:10.1140/epjc/s10052-018-5530-9.

[376] R. Aaij, et al., Observation of the decay $\Lambda_{b}^{0} \rightarrow \psi(2 S) p \pi^{-}$, JHEP 08 (2018) 131. arXiv:1806. 08084, doi:10.1007/JHEP08(2018) 131.

[377] G.-N. Li, X.-G. He, M. He, Some Predictions of Diquark Model for Hidden Charm Pentaquark Discovered at the LHCb, JHEP 12 (2015) 128. arXiv:1507.08252, doi:10.1007/JHEP12(2015) 128.

[378] R. Ghosh, A. Bhattacharya, B. Chakrabarti, A study on $\mathrm{P}_{c}^{*}(4380)$ and $\mathrm{P}_{c}^{*}$ in the quasi particle diquark model[Phys. Part. Nucl. Lett.14,no.4,550(2017)]. arXiv:1508.00356, doi:10.1134/ S1547477117040100.

[379] H.-X. Chen, W. Chen, S.-L. Zhu, Possible interpretations of the $P_{c}(4312), P_{c}(4440)$, and $P_{c}(4457)$ arXiv: 1903.11001.

[380] R. Chen, X. Liu, Z.-F. Sun, S.-L. Zhu, Strong LHCb evidence for supporting the existence of hidden-charm molecular pentaquarksarXiv:1903.11013.

[381] M.-Z. Liu, Y.-W. Pan, F.-Z. Peng, M. S. Sanchez, L.-S. Geng, A. Hosaka, M. P. Valderrama, Emergence of a complete heavy-quark spin symmetry multiplet: seven molecular pentaquarks in light of the latest LHCb analysisarXiv:1903.11560.

[382] F.-K. Guo, H.-J. Jing, U.-G. Meisser, S. Sakai, Isospin breaking decays as a diagnosis of the hadronic molecular structure of the $P_{c}(4457)$ arXiv:1903.11503.

[383] J. Hofmann, M. F. M. Lutz, D-wave baryon resonances with charm from coupled-channel dynamics, Nucl. Phys. A776 (2006) 17-51. arXiv:hep-ph/0601249, doi:10.1016/j.nuclphysa. 2006. 07.004 .

[384] F.-K. Guo, U.-G. Meissner, More kaonic bound states and a comprehensive interpretation of the $D_{s J}$ states, Phys. Rev. D84 (2011) 014013. arXiv:1102.3536, doi:10.1103/PhysRevD.84. 014013.

[385] O. Romanets, L. Tolos, C. Garcia-Recio, J. Nieves, L. L. Salcedo, R. G. E. Timmermans, Charmed and strange baryon resonances with heavy-quark spin symmetry, Phys. Rev. D85 (2012) 114032. arXiv:1202.2239, doi:10.1103/PhysRevD.85.114032.

[386] J. M. Dias, V. R. Debastiani, J. J. Xie, E. Oset, Doubly charmed $\Xi_{c c}$ molecular states from meson-baryon interaction, Phys. Rev. D98 (9) (2018) 094017. arXiv:1805.03286, doi:10.1103/ PhysRevD.98.094017.

[387] Q. Xu, G. Liu, H. Jin, Possible bound state of the double heavy meson-baryon system, Phys. Rev. D86 (2012) 114032. arXiv:1012.5949, doi:10.1103/PhysRevD.86.114032.

[388] Z.-H. Guo, Prediction of exotic doubly charmed baryons within chiral effective field theory, Phys. Rev. D96 (7) (2017) 074004. arXiv:1708.04145, doi:10.1103/PhysRevD.96.074004.

[389] R. Chen, F.-L. Wang, A. Hosaka, X. Liu, Exotic triple-charm deuteronlike hexaquarks, Phys. Rev. D97 (11) (2018) 114011. arXiv:1804.02961, doi:10.1103/PhysRevD.97.114011.

[390] R. Chen, A. Hosaka, X. Liu, Prediction of triple-charm molecular pentaquarks, Phys. Rev. D96 (11) (2017) 114030. arXiv:1711.09579, doi:10.1103/PhysRevD.96.114030. 
[391] F.-L. Wang, R. Chen, Z.-W. Liu, X. Liu, Possible triple-charm molecular pentaquarks from $\Xi_{c c} D_{1} / \Xi_{c c} D_{2}^{*}$ interactions, Phys. Rev. D99 (5) (2019) 054021. arXiv:1901.01542, doi:10.1103/ PhysRevD.99.054021.

[392] A. V. Manohar, M. B. Wise, Heavy quark physics, Camb. Monogr. Part. Phys. Nucl. Phys. Cosmol. 10 (2000) 1-191.

[393] A. Hosaka, T. Hyodo, K. Sudoh, Y. Yamaguchi, S. Yasui, Heavy Hadrons in Nuclear Matter, Prog. Part. Nucl. Phys. 96 (2017) 88-153. arXiv:1606.08685, doi:10.1016/j.ppnp.2017.04.003.

[394] M. J. Savage, M. B. Wise, Spectrum of baryons with two heavy quarks, Phys. Lett. B248 (1990) 177-180. doi:10.1016/0370-2693(90)90035-5.

[395] N. Brambilla, A. Vairo, T. Rosch, Effective field theory Lagrangians for baryons with two and three heavy quarks, Phys. Rev. D72 (2005) 034021. arXiv:hep-ph/0506065, doi:10.1103/PhysRevD. 72.034021.

[396] S. Fleming, T. Mehen, Doubly heavy baryons, heavy quark-diquark symmetry and NRQCD, Phys. Rev. D73 (2006) 034502. arXiv: hep-ph/0509313, doi:10.1103/PhysRevD.73.034502.

[397] T. D. Cohen, P. M. Hohler, Doubly heavy hadrons and the domain of validity of doubly heavy diquark-anti-quark symmetry, Phys. Rev. D74 (2006) 094003. arXiv:hep-ph/0606084, doi: 10.1103/PhysRevD.74.094003.

[398] Y. Cai, T. Cohen, On the existence of near threshold exotic hadrons containing two heavy quarksarXiv: 1901.05473.

[399] M. Cleven, F.-K. Guo, C. Hanhart, Q. Wang, Q. Zhao, Employing spin symmetry to disentangle different models for the XYZ states, Phys. Rev. D92 (1) (2015) 014005. arXiv:1505.01771, doi:10.1103/PhysRevD.92.014005.

[400] V. Baru, E. Epelbaum, A. A. Filin, C. Hanhart, U.-G. Meissner, A. V. Nefediev, Heavy-quark spin symmetry partners of the X (3872) revisited, Phys. Lett. B763 (2016) 20-28. arXiv:1605.09649, doi:10.1016/j.physletb.2016.10.008.

[401] V. Baru, E. Epelbaum, A. A. Filin, C. Hanhart, A. V. Nefediev, Spin partners of the Z $Z_{b}(10610)$ and $\mathrm{Z}_{b}$ (10650) revisited, JHEP 06 (2017) 158. arXiv:1704.07332, doi:10.1007/JHEP06 (2017) 158.

[402] M.-Z. Liu, T.-W. Wu, M. P. Valderrama, J.-J. Xie, L.-S. Geng, Heavy-quark spin and flavour symmetry partners of the $\mathrm{X}(3872)$ revisited: what can we learn from the one boson exchange model?arXiv: 1902.03044.

[403] M. T. AlFiky, F. Gabbiani, A. A. Petrov, X(3872): Hadronic molecules in effective field theory, Phys. Lett. B640 (2006) 238-245. arXiv:hep-ph/0506141, doi:10.1016/j.physletb.2006.07. 069.

[404] G. Chiladze, A. F. Falk, A. A. Petrov, Hybrid charmonium production in B decays, Phys. Rev. D58 (1998) 034013. arXiv:hep-ph/9804248, doi:10.1103/PhysRevD.58.034013.

[405] A. A. Petrov, X(3872), X(3940) as hybrid charmonium states?, J. Phys. Conf. Ser. 9 (2005) 83-86. doi:10.1088/1742-6596/9/1/013.

[406] M. B. Voloshin, Light Quark Spin Symmetry in $Z_{b}$ Resonances?, Phys. Rev. D93 (7) (2016) 074011. arXiv:1601.02540, doi:10.1103/PhysRevD .93.074011. 
[407] J. Hu, T. Mehen, Chiral Lagrangian with heavy quark-diquark symmetry, Phys. Rev. D73 (2006) 054003. arXiv:hep-ph/0511321, doi:10.1103/PhysRevD.73.054003.

[408] Y. Shimizu, Y. Yamaguchi, M. Harada, Heavy quark spin multiplet structure of $\bar{P}^{(*)} \Sigma_{Q}^{(*)}$ molecular states, Phys. Rev. D98 (1) (2018) 014021. arXiv:1805.05740, doi:10.1103/PhysRevD.98. 014021.

[409] Y. Shimizu, Y. Yamaguchi, M. Harada, Heavy quark spin multiplet structure of $P_{c}$-like pentaquark as P-wave hadronic molecular statearXiv:1901.09215.

[410] M.-Z. Liu, F.-Z. Peng, M. Snchez Snchez, M. P. Valderrama, Heavy-quark symmetry partners of the $P_{c}(4450)$ pentaquark, Phys. Rev. D98 (11) (2018) 114030. arXiv:1811.03992, doi:10.1103/ PhysRevD.98.114030.

[411] M. A. Shifman, A. I. Vainshtein, V. I. Zakharov, QCD and Resonance Physics. Theoretical Foundations, Nucl. Phys. B147 (1979) 385-447. doi:10.1016/0550-3213(79)90022-1.

[412] L. J. Reinders, H. Rubinstein, S. Yazaki, Hadron Properties from QCD Sum Rules, Phys. Rept. 127 (1985) 1. doi:10.1016/0370-1573(85)90065-1.

[413] H.-X. Chen, W. Chen, X. Liu, Y.-R. Liu, S.-L. Zhu, A review of the open charm and open bottom systems, Rept. Prog. Phys. 80 (7) (2017) 076201. arXiv:1609.08928, doi:10.1088/1361-6633/ aa6420.

[414] P. Colangelo, A. Khodjamirian, QCD sum rules, a modern perspective, arXiv:hep-ph/0010175.

[415] S. Narison, QCD as a theory of hadrons from partons to confinement, arXiv:hep-ph/0205006.

[416] M. Nielsen, F. S. Navarra, S. H. Lee, New Charmonium States in QCD Sum Rules: A Concise Review, Phys. Rept. 497 (2010) 41-83. arXiv:0911.1958, doi:10.1016/j.physrep.2010.07. 005.

[417] R. M. Albuquerque, J. M. Dias, K. P. Khemchandani, A. Martinez Torres, F. S. Navarra, M. Nielsen, C. M. Zanetti, QCD Sum Rules Approach to the $X, Y$ and $Z$ StatesarXiv: 1812.08207.

[418] H.-X. Chen, C.-P. Shen, S.-L. Zhu, A possible partner state of the $Y(2175)$, Phys. Rev. D98 (1) (2018) 014011. arXiv:1805.06100, doi:10.1103/PhysRevD.98.014011.

[419] E.-L. Cui, H.-M. Yang, H.-X. Chen, W. Chen, C.-P. Shen, QCD sum rule studies of $s s \bar{s} \bar{s}$ tetraquark states with $J^{P C}=1^{+-}$, Eur. Phys. J. C79 (3) (2019) 232. arXiv:1901.01724, doi:10.1140/ epjc/s10052-019-6755-y.

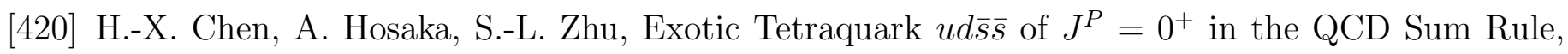
Phys. Rev. D74 (2006) 054001. arXiv:hep-ph/0604049, doi:10.1103/PhysRevD.74.054001.

[421] M. Fierz, Z. Physik 104 (1937) 553.

[422] H.-S. Zong, F. Wang, J.-L. Ping, The Generalized Fierz transformation and its application, Commun. Theor. Phys. 22 (1994) 479-482.

[423] J. A. Maruhn, T. Buervenich, D. G. Madland, Calculating the Fierz transformation for higher orders, Chin. J. Comput. Phys. 169 (2001) 238. arXiv:nucl-th/0007010, doi:10.1006/jcph. 2001.6727 . 
[424] H.-X. Chen, A. Hosaka, S.-L. Zhu, QCD sum rule study of the masses of light tetraquark scalar mesons, Phys. Lett. B650 (2007) 369-372. arXiv:hep-ph/0609163, doi:10.1016/j.physletb. 2007.05 .031$.

[425] H.-X. Chen, A. Hosaka, S.-L. Zhu, Light Scalar Tetraquark Mesons in the QCD Sum Rule, Phys. Rev. D76 (2007) 094025. arXiv:0707.4586, doi:10.1103/PhysRevD.76.094025.

[426] H.-X. Chen, A. Hosaka, S.-L. Zhu, Light Scalar Mesons in the QCD Sum Rule, Prog. Theor. Phys. Suppl. 168 (2007) 186-189. arXiv:0711.1007, doi:10.1143/PTPS.168.186.

[427] H.-X. Chen, A. Hosaka, S.-L. Zhu, The $I^{G} J^{P C}=1^{-} 1^{-+}$Tetraquark States, Phys. Rev. D78 (2008) 054017. arXiv:0806.1998, doi:10.1103/PhysRevD.78.054017.

[428] H.-X. Chen, A. Hosaka, S.-L. Zhu, Scalar Tetraquark Currents With Application to the QCD Sum Rule, Mod. Phys. Lett. A23 (2008) 2234-2237. arXiv:0811.1514, doi:10.1142/ S0217732308029095.

[429] H.-X. Chen, A. Hosaka, S.-L. Zhu, The $I^{G} J^{P C}=0^{+} 1^{-+}$Tetraquark State, Phys. Rev. D78 (2008) 117502. arXiv:0808.2344, doi:10.1103/PhysRevD.78.117502.

[430] C.-K. Jiao, W. Chen, H.-X. Chen, S.-L. Zhu, The Possible $J^{P C}=0^{--}$Exotic State, Phys. Rev. D79 (2009) 114034. arXiv:0905.0774, doi:10.1103/PhysRevD.79.114034.

[431] H.-X. Chen, A. Hosaka, H. Toki, S.-L. Zhu, Light Scalar Meson sigma(600) in QCD Sum Rule with Continuum, Phys. Rev. D81 (2010) 114034. arXiv:0912.5138, doi:10.1103/PhysRevD. 81.114034.

[432] W. Chen, S.-L. Zhu, The Possible $J^{P C}=0^{--}$Charmonium-like State, Phys. Rev. D81 (2010) 105018. arXiv:1003.3721, doi:10.1103/PhysRevD.81.105018.

[433] W. Chen, Z.-X. Cai, S.-L. Zhu, Masses of the tensor mesons with $J^{P}=2^{-}$, Nucl. Phys. B887 (2014) 201-215. arXiv:1107.4949, doi:10.1016/j.nuclphysb.2014.08.006.

[434] M.-L. Du, W. Chen, X.-L. Chen, S.-L. Zhu, The Possible $J^{P C}=0^{+-}$Exotic State, Chin.Phys. C37 (2013) 033104. arXiv:1203.5199, doi:10.1088/1674-1137/37/3/033104.

[435] H.-X. Chen, The "Closed" Chiral Symmetry and Its Application to Tetraquark, Eur. Phys. J. C72 (2012) 2204. arXiv:1210.3399, doi:10.1140/epjc/s10052-012-2204-x.

[436] H.-X. Chen, Chiral Structure of Vector and Axial-Vector Tetraquark Currents, Eur. Phys. J. C73 (2013) 2628. arXiv:1311.4992, doi:10.1140/epjc/s10052-013-2628-y.

[437] H.-X. Chen, Chiral Structure of Scalar and Pseudoscalar Mesons, Adv. High Energy Phys. 2013 (2013) 750591. arXiv:1311.4434, doi:10.1155/2013/750591.

[438] H.-X. Chen, E.-L. Cui, W. Chen, T. G. Steele, S.-L. Zhu, QCD sum rule study of the $d^{*}(2380)$, Phys. Rev. C91 (2) (2015) 025204. arXiv:1410.0394, doi:10.1103/PhysRevC.91.025204.

[439] H.-X. Chen, E.-L. Cui, W. Chen, T. G. Steele, X. Liu, S.-L. Zhu, $a_{1}(1420)$ resonance as a tetraquark state and its isospin partner, Phys. Rev. D91 (2015) 094022. arXiv:1503.02597, doi:10.1103/PhysRevD.91.094022.

[440] H.-X. Chen, D. Zhou, W. Chen, X. Liu, S.-L. Zhu, Searching for hidden-charm baryonium signals in QCD sum rules, Eur. Phys. J. C76 (11) (2016) 602. arXiv:1605.07453, doi:10.1140/epjc/ s10052-016-4459-0. 
[441] H.-X. Chen, V. Dmitrasinovic, A. Hosaka, K. Nagata, S.-L. Zhu, Chiral Properties of Baryon Fields with Flavor SU(3) Symmetry, Phys. Rev. D78 (2008) 054021. arXiv:0806.1997, doi: 10.1103/PhysRevD.78.054021.

[442] H.-X. Chen, V. Dmitrasinovic, A. Hosaka, Baryon fields with $U_{L}(3) \times U_{R}(3)$ chiral symmetry II: Axial currents of nucleons and hyperons, Phys. Rev. D81 (2010) 054002. arXiv:0912.4338, doi:10.1103/PhysRevD.81.054002.

[443] H.-X. Chen, V. Dmitrasinovic, A. Hosaka, Baryon Fields with $U_{L}(3)$ times $U_{R}(3)$ Chiral Symmetry III: Interactions with Chiral $(3, \overline{3})+(\overline{3}, 3)$ Spinless Mesons, Phys. Rev. D83 (2011) 014015. arXiv: 1009.2422, doi:10.1103/PhysRevD.83.014015.

[444] V. Dmitrasinovic, H.-X. Chen, Bi-local baryon interpolating fields with two flavours, Eur. Phys. J. C71 (2011) 1543. arXiv:1101.5906, doi:10.1140/epjc/s10052-011-1543-3.

[445] H.-X. Chen, V. Dmitrasinovic, A. Hosaka, Baryons with $U_{L}(3) \times U_{R}(3)$ Chiral Symmetry IV: Interactions with Chiral $(8,1)+(1,8)$ Vector and Axial-vector Mesons and Anomalous Magnetic Moments, Phys. Rev. C85 (2012) 055205. arXiv:1109.3130, doi:10.1103/PhysRevC.85.055205.

[446] H.-X. Chen, Chiral Baryon Fields in the QCD Sum Rule, Eur. Phys. J. C72 (2012) 2180. arXiv: 1203.3260, doi:10.1140/epjc/s10052-012-2180-1.

[447] H.-X. Chen, Baryon Tri-local Interpolating Fields, Eur. Phys. J. C72 (2012) 2129. arXiv: 1205. 5328, doi:10.1140/epjc/s10052-012-2129-4.

[448] H.-X. Chen, V. Dmitrasinovic, Bilocal baryon interpolating fields with three flavors, Phys. Rev. D88 (3) (2013) 036013. arXiv:1309.0387, doi:10.1103/PhysRevD.88.036013.

[449] V. Dmitrašinović, H.-X. Chen, A. Hosaka, Baryon fields with $U_{L}(3) \times U_{R}(3)$ chiral symmetry. V. Pion-nucleon and kaon-nucleon $\sigma$ terms, Phys. Rev. C93 (6) (2016) 065208. arXiv:1812.03414, doi:10.1103/PhysRevC.93.065208.

[450] H.-X. Chen, Q. Mao, W. Chen, X. Liu, S.-L. Zhu, Establishing low-lying doubly charmed baryons, Phys. Rev. D96 (3) (2017) 031501, [Erratum: Phys. Rev.D96,no.11,119902(2017)]. arXiv:1707. 01779, doi:10.1103/PhysRevD.96.031501,10.1103/PhysRevD.96.119902.

[451] E.-L. Cui, H.-X. Chen, W. Chen, X. Liu, S.-L. Zhu, Suggested search for doubly charmed baryons of $J^{P}=3 / 2^{+}$via their electromagnetic transitions, Phys. Rev. D97 (3) (2018) 034018. arXiv: 1712.03615, doi:10.1103/PhysRevD.97.034018.

[452] X. Liu, H.-X. Chen, Y.-R. Liu, A. Hosaka, S.-L. Zhu, Bottom baryons, Phys. Rev. D77 (2008) 014031. arXiv:0710.0123, doi:10.1103/PhysRevD.77.014031.

[453] P.-Z. Huang, H.-X. Chen, S.-L. Zhu, Light vector meson and heavy baryon strong interaction, Phys. Rev. D80 (2009) 094007. arXiv:0909.5551, doi:10.1103/PhysRevD.80.094007.

[454] H.-X. Chen, W. Chen, Q. Mao, A. Hosaka, X. Liu, S.-L. Zhu, P-wave charmed baryons from QCD sum rules, Phys. Rev. D91 (5) (2015) 054034. arXiv:1502.01103, doi:10.1103/PhysRevD.91. 054034.

[455] Q. Mao, H.-X. Chen, W. Chen, A. Hosaka, X. Liu, S.-L. Zhu, QCD sum rule calculation for P-wave bottom baryons, Phys. Rev. D92 (2015) 114007. arXiv:1510.05267, doi:10.1103/PhysRevD. 92.114007. 
[456] H.-X. Chen, Q. Mao, A. Hosaka, X. Liu, S.-L. Zhu, D-wave charmed and bottomed baryons from QCD sum rules, Phys. Rev. D94 (11) (2016) 114016. arXiv:1611.02677, doi:10.1103/ PhysRevD.94.114016.

[457] Q. Mao, H.-X. Chen, A. Hosaka, X. Liu, S.-L. Zhu, D-wave heavy baryons of the $S U(3)$ flavor 6 $_{F}$, Phys. Rev. D96 (7) (2017) 074021. arXiv:1707.03712, doi:10.1103/PhysRevD.96.074021.

[458] H.-X. Chen, Q. Mao, W. Chen, A. Hosaka, X. Liu, S.-L. Zhu, Decay properties of P-wave charmed baryons from light-cone QCD sum rules, Phys. Rev. D95 (9) (2017) 094008. arXiv:1703.07703, doi:10.1103/PhysRevD .95.094008.

[459] D. Zhou, E.-L. Cui, H.-X. Chen, L.-S. Geng, X. Liu, S.-L. Zhu, D-wave heavy-light mesons from QCD sum rules, Phys. Rev. D90 (11) (2014) 114035. arXiv:1410.1727, doi:10.1103/PhysRevD. 90.114035 .

[460] D. Zhou, H.-X. Chen, L.-S. Geng, X. Liu, S.-L. Zhu, F-wave heavy-light meson spectroscopy in QCD sum rules and heavy quark effective theory, Phys. Rev. D92 (11) (2015) 114015. arXiv: 1506.00766, doi:10.1103/PhysRevD.92.114015.

[461] R. Aaij, et al., Observation of five new narrow $\Omega_{c}^{0}$ states decaying to $\Xi_{c}^{+} K^{-}$, Phys. Rev. Lett. 118 (18) (2017) 182001. arXiv:1703.04639, doi:10.1103/PhysRevLett.118.182001.

[462] W. Chen, S.-L. Zhu, The Vector and Axial-Vector Charmonium-like States, Phys. Rev. D83 (2011) 034010. arXiv:1010.3397, doi:10.1103/PhysRevD.83.034010.

[463] C. Z. Yuan, et al., Measurement of $e^{+} e^{-} \rightarrow \pi^{+} \pi^{-} J / \psi$ cross-section via initial state radiation at Belle, Phys. Rev. Lett. 99 (2007) 182004. arXiv:0707.2541, doi:10.1103/PhysRevLett.99. 182004.

[464] B. Aubert, et al., Observation of a broad structure in the $\pi^{+} \pi^{-} J / \psi$ mass spectrum around $4.26 \mathrm{GeV} / \mathrm{c}^{2}$, Phys. Rev. Lett. 95 (2005) 142001. arXiv:hep-ex/0506081, doi:10.1103/ PhysRevLett.95.142001.

[465] B. Aubert, et al., Evidence of a broad structure at an invariant mass of $4.32 \mathrm{GeV} / \mathrm{c}^{2}$ in the reaction $e^{+} e^{-} \rightarrow \pi^{+} \pi^{-} \psi(2 S)$ measured at BaBar, Phys. Rev. Lett. 98 (2007) 212001. arXiv: hep-ex/0610057, doi:10.1103/PhysRevLett.98.212001.

[466] G. Pakhlova, et al., Observation of a near-threshold enhancement in the $e^{+} e^{-} \rightarrow \Lambda_{c}^{+} \Lambda_{c}^{-}$cross section using initial-state radiation, Phys. Rev. Lett. 101 (2008) 172001. arXiv:0807.4458, doi: 10.1103/PhysRevLett.101.172001.

[467] X. L. Wang, et al., Observation of Two Resonant Structures in $e^{+} e^{-} \rightarrow \pi^{+} \pi^{-} \psi(2 S)$ via Initial State Radiation at Belle, Phys. Rev. Lett. 99 (2007) 142002. arXiv:0707.3699, doi:10.1103/ PhysRevLett.99.142002.

[468] H.-X. Chen, E.-L. Cui, W. Chen, X. Liu, T. G. Steele, S.-L. Zhu, QCD sum rule study of hiddencharm pentaquarks, Eur. Phys. J. C76 (10) (2016) 572. arXiv:1602.02433, doi:10.1140/epjc/ s10052-016-4438-5.

[469] J.-B. Xiang, H.-X. Chen, W. Chen, X.-B. Li, X.-Q. Yao, S.-L. Zhu, Revisiting hidden-charm pentaquarks from QCD sum rules, Chin. Phys. C43 (2019) 034104. arXiv:1711.01545, doi: $10.1088 / 1674-1137 / 43 / 3 / 034104$. 
[470] H.-X. Chen, X. Liu, A. Hosaka, S.-L. Zhu, The Y(2175) State in the QCD Sum Rule, Phys. Rev. D78 (2008) 034012. arXiv:0801.4603, doi:10.1103/PhysRevD.78.034012.

[471] B. Aubert, et al., The $e^{+} e^{-} \rightarrow K^{+} K^{-} \pi^{+} \pi^{-}, K^{+} K^{-} \pi^{0} \pi^{0}$ and $K^{+} K^{-} K^{+} K^{-}$cross-sections measured with initial-state radiation, Phys. Rev. D76 (2007) 012008. arXiv:0704.0630, doi: 10.1103/PhysRevD.76.012008.

[472] C. P. Shen, et al., Observation of the $\phi(1680)$ and the $Y(2175)$ in $e^{+} e^{-} \rightarrow \phi \pi^{+} \pi^{-}$, Phys. Rev. D80 (2009) 031101. arXiv:0808.0006, doi:10.1103/PhysRevD.80.031101.

[473] C. P. Shen, C. Z. Yuan, Combined fit to BaBar and Belle Data on $e^{+} e^{-} \rightarrow \phi \pi^{+} \pi^{-}$and phi $f_{0}(980)$, Chin. Phys. C34 (2010) 1045-1051. arXiv:0911.1591, doi:10.1088/1674-1137/34/8/002.

[474] M. Ablikim, et al., Observation and study of $J / \psi \rightarrow \phi \eta \eta^{\prime}$ at BESIIIarXiv:1901.00085.

[475] H.-X. Chen, W. Chen, Settling the $Z_{c}(4600)$ in the charged charmonium-like familyarXiv:1901. 06946.

[476] R. Aaij, et al., Model-independent observation of exotic contributions to $B^{0} \rightarrow J / \psi K^{+} \pi^{-}$decaysarXiv: 1901.05745.

[477] R. D. Matheus, S. Narison, M. Nielsen, J. M. Richard, Can the X(3872) be a $1^{++}$four-quark state?, Phys. Rev. D75 (2007) 014005. arXiv:hep-ph/0608297, doi:10.1103/PhysRevD.75.014005.

[478] F. S. Navarra, M. Nielsen, $X(3872) \rightarrow J / \psi \pi^{+} \pi^{-}$and $X(3872) \rightarrow J / \psi \pi^{+} \pi^{-} \pi^{0}$ decay widths from QCD sum rules, Phys. Lett. B639 (2006) 272-277. arXiv:hep-ph/0605038, doi:10.1016/j. physletb.2006.06.054.

[479] S. H. Lee, A. Mihara, F. S. Navarra, M. Nielsen, QCD sum rules study of the meson $Z^{+}(4430)$, Phys. Lett. B661 (2008) 28-32. arXiv:0710.1029, doi:10.1016/j.physletb.2008.01.062.

[480] M. E. Bracco, S. H. Lee, M. Nielsen, R. Rodrigues da Silva, The Meson $Z^{+}(4430)$ as a tetraquark state, Phys. Lett. B671 (2009) 240-244. arXiv:0807.3275, doi:10.1016/j.physletb.2008.12. 021.

[481] R. M. Albuquerque, M. Nielsen, QCD sum rules study of the $J^{P C}=1^{--}$charmonium $Y$ mesons, Nucl. Phys. A815 (2009) 53-66, [Erratum: Nucl. Phys. A857, 48 (2011)]. arXiv:0804.4817, doi:10.1016/j.nuclphysa.2011.04.001,10.1016/j.nuclphysa.2008.10.015.

[482] R. D. Matheus, F. S. Navarra, M. Nielsen, C. M. Zanetti, QCD Sum Rules for the X(3872) as a mixed molecule-charmoniun state, Phys. Rev. D80 (2009) 056002. arXiv:0907.2683, doi: 10.1103/PhysRevD.80.056002.

[483] R. M. Albuquerque, M. E. Bracco, M. Nielsen, A QCD sum rule calculation for the Y(4140) narrow structure, Phys. Lett. B678 (2009) 186-190. arXiv:0903.5540, doi:10.1016/j.physletb. 2009. 06.022 .

[484] Z.-G. Wang, Analysis of the $X(4350)$ as a scalar $\bar{c} c$ and $D_{s}^{*} \bar{D}_{s}^{*}$ mixing state with QCD sum rules, Phys. Lett. B690 (2010) 403-406. arXiv:0912.4626, doi:10.1016/j.physletb.2010.05.068.

[485] Z.-G. Wang, Analysis of the Y(4140) with QCD sum rules, Eur. Phys. J. C63 (2009) 115-122. arXiv:0903.5200, doi:10.1140/epjc/s10052-009-1097-9.

[486] J.-R. Zhang, M.-Q. Huang, $(q \bar{s})^{(*)}(\bar{q} s)^{(*)}$ molecular states from qcd sum rules: A view on $y(4140)$, J. Phys. G37 (2010) 025005. arXiv:0905.4178, doi:10.1088/0954-3899/37/2/025005. 
[487] J.-R. Zhang, M.-Q. Huang, Could $Y_{b}(10890)$ be the P-wave $[b q][\bar{b} \bar{q}]$ tetraquark state?, JHEP 11 (2010) 057. arXiv:1011.2815, doi:10.1007/JHEP11(2010) 057.

[488] J.-R. Zhang, M.-Q. Huang, The P-wave $[c s][\bar{c} \bar{s}]$ tetraquark state: $Y(4260)$ or $Y(4660)$ ?, Phys. Rev. D83 (2011) 036005. arXiv:1011.2818, doi:10.1103/PhysRevD.83.036005.

[489] J.-R. Zhang, M. Zhong, M.-Q. Huang, Could $Z_{b}(10610)$ be a $B^{*} \bar{B}$ molecular state?, Phys. Lett. B704 (2011) 312-315. arXiv:1105.5472, doi:10.1016/j.physletb.2011.09.039.

[490] W. Chen, S.-L. Zhu, Spin-1 charmonium-like states in QCD sum rule, EPJ Web Conf. 20 (2012) 01003. arXiv:1209.4748, doi:10.1051/epjconf/20122001003.

[491] W. Chen, T. G. Steele, M.-L. Du, S.-L. Zhu, $D^{*} \bar{D}^{*}$ molecule interpretation of $Z_{c}(4025)$, Eur. Phys. J. C74 (2) (2014) 2773. arXiv:1308.5060, doi:10.1140/epjc/s10052-014-2773-y.

[492] W. Chen, W.-Z. Deng, J. He, N. Li, X. Liu, Z.-G. Luo, Z.-F. Sun, S.-L. Zhu, XYZ States, PoS Hadron2013 (2013) 005. arXiv:1311.3763, doi:10.22323/1.205.0005.

[493] Z.-G. Wang, Analysis of the $Z_{c}(4020), Z_{c}(4025), Y(4360)$ and $Y(4660)$ as vector tetraquark states with QCD sum rules, Eur. Phys. J. C74 (5) (2014) 2874. arXiv:1311.1046, doi:10.1140/epjc/ s10052-014-2874-7.

[494] Z.-G. Wang, T. Huang, Possible assignments of the $X(3872), Z_{c}(3900)$ and $Z_{b}(10610)$ as axialvector molecular states, Eur. Phys. J. C74 (5) (2014) 2891. arXiv:1312.7489, doi:10.1140/ epjc/s10052-014-2891-6.

[495] W. Chen, T. G. Steele, S.-L. Zhu, Heavy tetraquark states and quarkonium hybrids, The Universe 2 (2014) 13-40. arXiv:1403.7457.

[496] W. Chen, T. G. Steele, H.-X. Chen, S.-L. Zhu, $Z_{c}(4200)^{+}$decay width as a charmonium-like tetraquark state, Eur. Phys. J. C75 (8) (2015) 358. arXiv:1501.03863, doi:10.1140/epjc/ s10052-015-3578-3.

[497] W. Chen, T. G. Steele, H.-X. Chen, S.-L. Zhu, Mass spectra of $Z_{c}$ and $Z_{b}$ exotic states as hadron molecules, Phys. Rev. D92 (5) (2015) 054002. arXiv:1505.05619, doi:10.1103/PhysRevD.92. 054002.

[498] Z.-G. Wang, Tetraquark state candidates: $Y(4260), Y(4360), Y(4660)$ and $Z_{c}(4020 / 4025)$, Eur. Phys. J. C76 (7) (2016) 387. arXiv:1601.05541, doi:10.1140/epjc/s10052-016-4238-y.

[499] Z.-R. Huang, W. Chen, T. G. Steele, Z.-F. Zhang, H.-Y. Jin, Investigation of the light fourquark states with exotic $J^{P C}=0^{--}$, Phys. Rev. D95 (7) (2017) 076017. arXiv:1610.02081, doi:10.1103/PhysRevD.95.076017.

[500] W. Chen, H.-X. Chen, X. Liu, T. G. Steele, S.-L. Zhu, Decoding the X(5568) as a fully openflavor sub $\bar{d}$ tetraquark state, Phys. Rev. Lett. 117 (2) (2016) 022002. arXiv:1602.08916, doi: 10.1103/PhysRevLett.117.022002.

[501] W. Chen, H.-X. Chen, X. Liu, T. G. Steele, S.-L. Zhu, Open-flavor charm and bottom $s q \bar{q} \bar{Q}$ and $q q \bar{q} \bar{Q}$ tetraquark states, Phys. Rev. D95 (11) (2017) 114005. arXiv:1705.10088, doi:10.1103/ PhysRevD.95.114005.

[502] Y.-C. Fu, Z.-R. Huang, Z.-F. Zhang, W. Chen, Exotic tetraquark states with $J^{P C}=0^{+-}$, Phys. Rev. D99 (1) (2019) 014025. arXiv:1811.03333, doi:10.1103/PhysRevD.99.014025. 
[503] J.-R. Zhang, Revisiting $D_{s 0}^{*}(2317)$ as a $0^{+}$tetraquark state from QCD sum rules, Phys. Lett. B789 (2019) 432-437. arXiv:1801.08725, doi:10.1016/j.physletb.2019.01.001.

[504] W. Chen, H.-X. Chen, X. Liu, T. G. Steele, S.-L. Zhu, Mass spectra for $q c \bar{q} \bar{c}, s c \bar{s} \bar{c}, q b \bar{q} \bar{b}, s b \bar{s} \bar{b}$ tetraquark states with $J^{P C}=0^{++}$and $2^{++}$, Phys. Rev. D96 (11) (2017) 114017. arXiv:1706. 09731, doi:10.1103/PhysRevD.96.114017.

[505] Z.-G. Wang, Analysis of the mass and width of the $X^{*}(3860)$ with QCD sum rules, Eur. Phys. J. A53 (10) (2017) 192. arXiv:1704.04111, doi:10.1140/epja/i2017-12390-6.

[506] Z.-G. Wang, Reanalysis of the $X(3915), X(4500)$ and $X(4700)$ with QCD sum rules, Eur. Phys. J. A53 (2) (2017) 19. arXiv:1607.04840, doi:10.1140/epja/i2017-12208-7.

[507] Z.-G. Wang, Reanalysis of $X(4140)$ as axial-vector tetraquark state with QCD sum rules, Eur. Phys. J. C76 (12) (2016) 657. arXiv:1607.00701, doi:10.1140/epjc/s10052-016-4515-9.

[508] S. S. Agaev, K. Azizi, H. Sundu, Exploring the resonances X(4140) and X(4274) through their decay channels, Phys. Rev. D95 (11) (2017) 114003. arXiv:1703.10323, doi:10.1103/PhysRevD. 95.114003.

[509] Z.-G. Wang, Analysis of the mass and width of the $Y(4274)$ as axialvector molecule-like state, Eur. Phys. J. C77 (3) (2017) 174. arXiv:1612.00195, doi:10.1140/epjc/s10052-017-4751-7.

[510] W. Chen, H.-X. Chen, X. Liu, T. G. Steele, S.-L. Zhu, Hunting for exotic doubly hiddencharm/bottom tetraquark states, Phys. Lett. B773 (2017) 247-251. arXiv:1605.01647, doi: $10.1016 / j$.physletb.2017.08.034.

[511] W. Chen, H.-X. Chen, X. Liu, T. G. Steele, S.-L. Zhu, Doubly hidden-charm/bottom $Q Q \bar{Q} \bar{Q}$ tetraquark states, EPJ Web Conf. 182 (2018) 02028. arXiv:1803.02522, doi:10.1051/epjconf/ 201818202028.

[512] Z.-G. Wang, Analysis of the $Q Q \bar{Q} \bar{Q}$ tetraquark states with QCD sum rules, Eur. Phys. J. C77 (7) (2017) 432. arXiv:1701.04285, doi:10.1140/epjc/s10052-017-4997-0.

[513] Z.-G. Wang, Z.-Y. Di, Analysis of the vector and axialvector $Q Q \bar{Q} \bar{Q}$ tetraquark states with QCD sum rulesarXiv: 1807.08520.

[514] J.-F. Jiang, W. Chen, S.-L. Zhu, Triply heavy $Q Q \bar{Q} \bar{q}$ tetraquark states, Phys. Rev. D96 (9) (2017) 094022. arXiv:1708.00142, doi:10.1103/PhysRevD.96.094022.

[515] M.-L. Du, W. Chen, X.-L. Chen, S.-L. Zhu, Exotic $Q Q \bar{q} \bar{q}, Q Q \bar{q} \bar{s}$ and $Q Q \bar{s} \bar{s}$ states, Phys. Rev. D87 (1) (2013) 014003. arXiv:1209.5134, doi:10.1103/PhysRevD.87.014003.

[516] F. S. Navarra, M. Nielsen, S. H. Lee, QCD sum rules study of $Q Q \bar{u} \bar{d}$ mesons, Phys. Lett. B649 (2007) 166-172. arXiv:hep-ph/0703071, doi:10.1016/j.physletb.2007.04.010.

[517] W. Chen, T. G. Steele, S.-L. Zhu, Exotic open-flavor $b c \bar{q} \bar{q}, b c \bar{s} \bar{s}$ and $q c \bar{q} \bar{b}, s c \bar{s} \bar{b}$ tetraquark states, Phys. Rev. D89 (5) (2014) 054037. arXiv:1310.8337, doi:10.1103/PhysRevD.89.054037.

[518] Z.-G. Wang, Z.-H. Yan, Analysis of the scalar, axialvector, vector, tensor doubly charmed tetraquark states with QCD sum rules, Eur. Phys. J. C78 (1) (2018) 19. arXiv:1710.02810, doi:10.1140/epjc/s10052-017-5507-0. 
[519] S. S. Agaev, K. Azizi, B. Barsbay, H. Sundu, The doubly charmed pseudoscalar tetraquarks $T_{c c ; \bar{s} \bar{s}}^{++}$ and $T_{c c ; \bar{d} \bar{s}}^{++}$, Nucl. Phys. B939 (2019) 130-144. arXiv:1806.04447, doi:10.1016/j.nuclphysb. 2018.12.021.

[520] S. S. Agaev, K. Azizi, B. Barsbay, H. Sundu, Weak decays of the axial-vector tetraquark $T_{b b ; \bar{u} \bar{d}}^{-}$, Phys. Rev. D99 (3) (2019) 033002. arXiv:1809.07791, doi:10.1103/PhysRevD.99.033002.

[521] H. Sundu, S. S. Agaev, K. Azizi, Semileptonic decays of the scalar tetraquark $Z_{b c ; \bar{u} \bar{d}}^{0} \operatorname{arXiv}: 1903$. 05931.

[522] K. Azizi, Y. Sarac, H. Sundu, Analysis of $P_{c}^{+}(4380)$ and $P_{c}^{+}(4450)$ as pentaquark states in the molecular picture with QCD sum rules, Phys. Rev. D95 (9) (2017) 094016. arXiv:1612.07479, doi:10.1103/PhysRevD.95.094016.

[523] K. Azizi, Y. Sarac, H. Sundu, Hidden Bottom Pentaquark States with Spin 3/2 and 5/2, Phys. Rev. D96 (9) (2017) 094030. arXiv:1707.01248, doi:10.1103/PhysRevD.96.094030.

[524] K. Azizi, Y. Sarac, H. Sundu, Strong decay of $P_{c}(4380)$ pentaquark in a molecular picture, Phys. Lett. B782 (2018) 694-701. arXiv:1802.01384, doi:10.1016/j.physletb.2018.06.022.

[525] U. Ozdem, K. Azizi, Electromagnetic multipole moments of the $P_{c}^{+}(4380)$ pentaquark in light-cone QCD, Eur. Phys. J. C78 (5) (2018) 379. arXiv:1803.06831, doi:10.1140/epjc/ s10052-018-5873-2.

[526] K. Azizi, U. özdem, The electromagnetic multipole moments of the possible charm-strange pentaquarks in light-cone QCD, Eur. Phys. J. C78 (9) (2018) 698. arXiv:1807.06503, doi: 10.1140/epjc/s10052-018-6187-0.

[527] Z.-G. Wang, Analysis of $P_{c}(4380)$ and $P_{c}(4450)$ as pentaquark states in the diquark model with QCD sum rules, Eur. Phys. J. C76 (2) (2016) 70. arXiv:1508.01468, doi:10.1140/epjc/ s10052-016-3920-4.

[528] Z.-G. Wang, T. Huang, Analysis of the $\frac{1}{2}^{ \pm}$pentaquark states in the diquark model with QCD sum rules, Eur. Phys. J. C76 (1) (2016) 43. arXiv:1508.04189, doi:10.1140/epjc/ s10052-016-3880-8.

[529] Z.-G. Wang, Analysis of the $\bar{D} \Sigma_{c}^{*}, \bar{D}^{*} \Sigma_{c}$ and $\bar{D}^{*} \Sigma_{c}^{*}$ pentaquark molecular states with QCD sum rulesarXiv: 1806.10384.

[530] Z.-G. Wang, Analysis of the doubly heavy baryon states and pentaquark states with QCD sum rules, Eur. Phys. J. C78 (10) (2018) 826. arXiv:1808.09820, doi:10.1140/epjc/ s10052-018-6300-4.

[531] A. Martinez Torres, K. P. Khemchandani, E. Oset, Three body resonances in two meson-one baryon systems, Phys. Rev. C77 (2008) 042203. arXiv:0706.2330, doi:10.1103/PhysRevC.77. 042203.

[532] A. Martinez Torres, K. P. Khemchandani, L. S. Geng, M. Napsuciale, E. Oset, The X(2175) as a resonant state of the phi K anti-K system, Phys. Rev. D78 (2008) 074031. arXiv:0801.3635, doi:10.1103/PhysRevD.78.074031.

[533] E. Epelbaum, A. Nogga, W. Gloeckle, H. Kamada, U. G. Meissner, H. Witala, Three nucleon forces from chiral effective field theory, Phys. Rev. C66 (2002) 064001. arXiv:nucl-th/0208023, doi:10.1103/PhysRevC.66.064001. 
[534] E. Epelbaum, H. Kamada, A. Nogga, H. Witala, W. Gloeckle, U.-G. Meissner, The Three nucleon and four nucleon systems from chiral effective field theory, Phys. Rev. Lett. 86 (2001) 4787-4790. arXiv:nucl-th/0007057, doi:10.1103/PhysRevLett.86.4787.

[535] V. Bernard, E. Epelbaum, H. Krebs, U.-G. Meissner, Subleading contributions to the chiral three-nucleon force. I. Long-range terms, Phys. Rev. C77 (2008) 064004. arXiv:0712.1967, doi:10.1103/PhysRevC.77.064004.

[536] V. Bernard, E. Epelbaum, H. Krebs, U. G. Meissner, Subleading contributions to the chiral threenucleon force II: Short-range terms and relativistic corrections, Phys. Rev. C84 (2011) 054001. arXiv:1108.3816, doi:10.1103/PhysRevC.84.054001.

[537] S. Petschauer, N. Kaiser, J. Haidenbauer, U.-G. Meissner, W. Weise, Leading three-baryon forces from SU(3) chiral effective field theory, Phys. Rev. C93 (1) (2016) 014001. arXiv:1511.02095, doi:10.1103/PhysRevC.93.014001.

[538] U.-G. Meissner, G. Ros, A. Rusetsky, Spectrum of three-body bound states in a finite volume, Phys. Rev. Lett. 114 (9) (2015) 091602, [Erratum: Phys. Rev. Lett.117,no.6,069902(2016)]. arXiv: 1412.4969, doi:10.1103/PhysRevLett.117.069902,10.1103/PhysRevLett.114.091602.

[539] M. Mai, M. Döring, Three-body Unitarity in the Finite Volume, Eur. Phys. J. A53 (12) (2017) 240. arXiv:1709.08222, doi:10.1140/epja/i2017-12440-1.

[540] Y. Meng, C. Liu, U.-G. Meissner, A. Rusetsky, Three-particle bound states in a finite volume: unequal masses and higher partial waves, Phys. Rev. D98 (1) (2018) 014508. arXiv:1712.08464, doi:10.1103/PhysRevD.98.014508.

[541] M. Döring, H. W. Hammer, M. Mai, J.-Y. Pang, A. Rusetsky, J. Wu, Three-body spectrum in a finite volume: the role of cubic symmetry, Phys. Rev. D97 (11) (2018) 114508. arXiv: 1802.03362, doi:10.1103/PhysRevD.97.114508.

[542] M. Mai, M. Doring, Finite-Volume Spectrum of $\pi^{+} \pi^{+}$and $\pi^{+} \pi^{+} \pi^{+}$Systems, Phys. Rev. Lett. 122 (6) (2019) 062503. arXiv:1807.04746, doi:10.1103/PhysRevLett.122.062503.

[543] R. A. Briceño, M. T. Hansen, S. R. Sharpe, Numerical study of the relativistic three-body quantization condition in the isotropic approximation, Phys. Rev. D98 (1) (2018) 014506. arXiv:1803.04169, doi:10.1103/PhysRevD.98.014506.

[544] R. A. Briceño, M. T. Hansen, S. R. Sharpe, Relating the finite-volume spectrum and the two-andthree-particle $S$ matrix for relativistic systems of identical scalar particles, Phys. Rev. D95 (7) (2017) 074510. arXiv:1701.07465, doi:10.1103/PhysRevD .95.074510.

[545] R. A. Briceno, Z. Davoudi, Three-particle scattering amplitudes from a finite volume formalism, Phys. Rev. D87 (9) (2013) 094507. arXiv:1212.3398, doi:10.1103/PhysRevD.87.094507.

[546] K. Polejaeva, A. Rusetsky, Three particles in a finite volume, Eur. Phys. J. A48 (2012) 67. arXiv:1203.1241, doi:10.1140/epja/i2012-12067-8.

[547] P. Guo, V. Gasparian, Numerical approach for finite volume three-body interaction, Phys. Rev. D97 (1) (2018) 014504. arXiv:1709.08255, doi:10.1103/PhysRevD.97.014504.

[548] P. Guo, V. Gasparian, An solvable three-body model in finite volume, Phys. Lett. B774 (2017) 441-445. arXiv:1701.00438, doi:10.1016/j.physletb.2017.10.009. 
[549] S. Kreuzer, H. W. Hammer, Efimov physics in a finite volume, Phys. Lett. B673 (2009) 260-263. arXiv:0811.0159, doi:10.1016/j.physletb.2009.02.035.

[550] M. Jansen, H. W. Hammer, Y. Jia, Finite volume corrections to the binding energy of the X(3872), Phys. Rev. D92 (11) (2015) 114031. arXiv:1505.04099, doi:10.1103/PhysRevD.92.114031.

[551] S. Bour, H. W. Hammer, D. Lee, U.-G. Meissner, Benchmark calculations for elastic fermiondimer scattering, Phys. Rev. C86 (2012) 034003. arXiv:1206.1765, doi:10.1103/PhysRevC. 86.034003 .

[552] H. W. Hammer, J. Y. Pang, A. Rusetsky, Three particle quantization condition in a finite volume: 2. general formalism and the analysis of data, JHEP 10 (2017) 115. arXiv:1707.02176, doi: 10.1007/JHEP10 (2017) 115.

[553] F. Romero-López, A. Rusetsky, C. Urbach, Two- and three-body interactions in $\varphi^{4}$ theory from lattice simulations, Eur. Phys. J. C78 (10) (2018) 846. arXiv:1806.02367, doi:10.1140/epjc/ s10052-018-6325-8.

[554] J.-Y. Pang, J.-J. Wu, H. W. Hammer, U.-G. Meissner, A. Rusetsky, Energy shift of the threeparticle system in a finite volumearXiv:1902.01111.

[555] L. D. Faddeev, Scattering theory for a three particle system, Sov. Phys. JETP 12 (1961) 10141019, [Zh. Eksp. Teor. Fiz.39,1459(1960)].

[556] W. Glöckle, The Quantum Mechanical Few-Body Problem, Springer-Verlag, 1983.

[557] A. Stadler, W. Glockle, P. U. Sauer, Faddeev equations with three-nucleon force in momentum space, Phys. Rev. C44 (1991) 2319-2327. doi:10.1103/PhysRevC.44.2319.

[558] H. Garcilazo, A. Valcarce, Deeply bound $\Xi$ tribaryon, Phys. Rev. C93 (3) (2016) 034001. arXiv: 1605.04108, doi:10.1103/PhysRevC.93.034001.

[559] H. Garcilazo, A. Valcarce, $\Omega N N$ and $\Omega \Omega N$ states, Phys. Rev. C99 (1) (2019) 014001. arXiv: 1901.05678, doi:10.1103/PhysRevC.99.014001.

[560] N. V. Shevchenko, A. Gal, J. Mares, J. Revai, anti-K NN quasi-bound state and the anti-K N interaction: Coupled-channel Faddeev calculations of the anti-K NN - pi Sigma N system, Phys. Rev. C76 (2007) 044004. arXiv:0706.4393, doi:10.1103/PhysRevC.76.044004.

[561] J. A. Oller, E. Oset, Chiral symmetry amplitudes in the S wave isoscalar and isovector channels and the $\sigma, \mathrm{f}_{0}(980), \mathrm{a}_{0}(980)$ scalar mesons, Nucl. Phys. A620 (1997) 438-456, [Erratum: Nucl. Phys.A652,407(1999)]. arXiv:hep-ph/9702314, doi:10.1016/S0375-9474(99)00427-3, 10.1016/S0375-9474(97)00160-7.

[562] L. Roca, E. Oset, J. Singh, Low lying axial-vector mesons as dynamically generated resonances, Phys. Rev. D72 (2005) 014002. arXiv:hep-ph/0503273, doi:10.1103/PhysRevD.72.014002.

[563] K. P. Khemchandani, A. Martinez Torres, E. Oset, The $N^{*}(1710)$ as a resonance in the $\pi \pi N$ system, Eur. Phys. J. A37 (2008) 233-243. arXiv:0804.4670, doi:10.1140/epja/i2008-10625-3.

[564] A. Martinez Torres, K. P. Khemchandani, D. Gamermann, E. Oset, The $Y(4260)$ as a $J / p s i K \bar{K}$ system, Phys. Rev. D80 (2009) 094012. arXiv:0906.5333, doi:10.1103/PhysRevD.80.094012.

[565] A. Martinez Torres, K. P. Khemchandani, L.-S. Geng, Bound state formation in the DDK systemarXiv:1809.01059. 
[566] A. Gal, H. Garcilazo, Coupled channel Faddeev calculations of a (Kbar-N-pi) quasibound state, Nucl. Phys. A864 (2011) 153-166. arXiv:1103.4757, doi:10.1016/j.nuclphysa.2011.06.022.

[567] M. P. Valderrama, Three-Body $J^{P}=0^{+}, 1^{+}, 2^{+} B^{*} B^{*} \bar{K}$ Bound States, Phys. Rev. D98 (1) (2018) 014022. arXiv:1805.05100, doi:10.1103/PhysRevD.98.014022.

[568] H. Garcilazo, A. Valcarce, $T_{b b b}$ : a three $B$-meson bound state, Phys. Lett. B784 (2018) 169-172. arXiv:1808.00226, doi:10.1016/j.physletb.2018.07.055.

[569] R. Chand, R. H. Dalitz, Charge-independence in K--deuterium capture reactions, Annals Phys. 20 (1962) 1-19. doi : 10.1016/0003-4916(62)90113-6.

[570] R. C. Barrett, A. Deloff, Strong interaction effects in kaonic deuterium, Phys. Rev. C60 (1999) 025201. doi:10.1103/PhysRevC.60.025201.

[571] A. Deloff, Eta d and K- d zero energy scattering: A Faddeev approach, Phys. Rev. C61 (2000) 024004. doi:10.1103/PhysRevC.61.024004.

[572] S. S. Kamalov, E. Oset, A. Ramos, Chiral unitary approach to the K- deuteron scattering length, Nucl. Phys. A690 (2001) 494-508. arXiv:nucl-th/0010054, doi:10.1016/S0375-9474(00) 00709-0.

[573] L. Roca, E. Oset, A description of the f2(1270), rho3(1690), f4(2050), rho5(2350) and f6(2510) resonances as multi-rho(770) states, Phys. Rev. D82 (2010) 054013. arXiv:1005.0283, doi: 10.1103/PhysRevD.82.054013.

[574] B.-X. Sun, H.-X. Chen, E. Oset, N-rho-rho and Delta-rho-rho molecules with $J^{P}=5 / 2^{+}$and $J^{P}=$ 7/2 , Eur. Phys. J. A47 (2011) 127. arXiv:1107.0209, doi:10.1140/epja/i2011-11127-y.

[575] E. Oset, A. Ramos, Dynamically generated resonances from the vector octet-baryon octet interaction, Eur. Phys. J. A44 (2010) 445-454. arXiv:0905.0973, doi:10.1140/epja/i2010-10957-3.

[576] R. E. Cutkosky, C. P. Forsyth, R. E. Hendrick, R. L. Kelly, Pion - Nucleon Partial Wave Amplitudes, Phys. Rev. D20 (1979) 2839. doi:10.1103/PhysRevD.20.2839.

[577] J. Yamagata-Sekihara, L. Roca, E. Oset, On the nature of the $K_{2}^{*}(1430), K_{3}^{*}(1780), K_{4}^{*}(2045)$, $K_{5}^{*}(2380)$ and $K^{*} 6$ as $K^{*}$ - multi- $\rho$ states, Phys. Rev. D82 (2010) 094017, [Erratum: Phys. Rev.D85,119905(2012)]. arXiv:1010.0525, doi:10.1103/PhysRevD.82.094017, 10.1103/ PhysRevD.85.119905.

[578] C. W. Xiao, M. Bayar, E. Oset, A prediction of $D^{*}$-multi- $\rho$ states, Phys. Rev. D86 (2012) 094019. arXiv:1207.4030, doi:10.1103/PhysRevD.86.094019.

[579] J.-J. Xie, A. Martinez Torres, E. Oset, Faddeev fixed center approximation to the $N \bar{K} K$ system and the signature of a $N^{*}(1920)\left(1 / 2^{+}\right)$state, Phys. Rev. C83 (2011) 065207. arXiv:1010.6164, doi:10.1103/PhysRevC.83.065207.

[580] W. Liang, C. W. Xiao, E. Oset, Study of $K \bar{K}$ and $K \bar{K}$ with the fixed center approximation to Faddeev equations, Phys. Rev. D88 (11) (2013) 114024. arXiv:1309.7310, doi:10.1103/ PhysRevD.88.114024.

[581] V. R. Debastiani, J. M. Dias, E. Oset, Study of the $D K K$ and $D K \bar{K}$ systems, Phys. Rev. D96 (1) (2017) 016014. arXiv:1705.09257, doi:10.1103/PhysRevD.96.016014. 
[582] M. Bayar, J. Yamagata-Sekihara, E. Oset, The $\bar{K} N N$ system with chiral dynamics, Phys. Rev. C84 (2011) 015209. arXiv:1102.2854, doi:10.1103/PhysRevC.84.015209.

[583] J. M. Dias, V. R. Debastiani, L. Roca, S. Sakai, E. Oset, On the binding of the $B D \bar{D}$ and $B D D$ systems, Phys. Rev. D96 (9) (2017) 094007. arXiv:1709.01372, doi:10.1103/PhysRevD.96. 094007.

[584] X.-L. Ren, B. B. Malabarba, L.-S. Geng, K. P. Khemchandani, A. Martnez Torres, $K^{*}$ mesons with hidden charm arising from $K X(3872)$ and $K Z_{c}(3900)$ dynamics, Phys. Lett. B785 (2018) 112-117. arXiv:1805.08330, doi:10.1016/j.physletb.2018.08.034.

[585] T. H. R. Skyrme, A Nonlinear field theory, Proc. Roy. Soc. Lond. A260 (1961) 127-138. doi: 10.1098/rspa.1961.0018.

[586] E. Witten, Baryons in the 1/n Expansion, Nucl. Phys. B160 (1979) 57-115. doi:10.1016/ 0550-3213(79) 90232-3.

[587] G. S. Adkins, C. R. Nappi, E. Witten, Static Properties of Nucleons in the Skyrme Model, Nucl. Phys. B228 (1983) 552. doi:10.1016/0550-3213(83)90559-X.

[588] S. J. Brodsky, J. R. Ellis, M. Karliner, Chiral Symmetry and the Spin of the Proton, Phys. Lett. B206 (1988) 309-315. doi:10.1016/0370-2693(88)91511-0.

[589] H. Weigel, Baryons as three flavor solitons, Int. J. Mod. Phys. A11 (1996) 2419-2544. arXiv: hep-ph/9509398, doi:10.1142/S0217751X96001218.

[590] D. Diakonov, V. Yu. Petrov, Chiral Theory of Nucleons, JETP Lett. 43 (1986) 75-77, [Pisma Zh. Eksp. Teor. Fiz.43,57(1986)].

[591] H. Reinhardt, R. Wunsch, Topological Solitons of the Nambu-Jona-Lasinio Model, Phys. Lett. B230 (1989) 93-98. doi:10.1016/0370-2693(89)91659-6.

[592] T. Meissner, F. Grummer, K. Goeke, Solitons in the Nambu-Jona-Lasinio Model, Phys. Lett. B227 (1989) 296-300. doi:10.1016/0370-2693(89)90932-5.

[593] M. Wakamatsu, H. Yoshiki, A Chiral quark model of the nucleon, Nucl. Phys. A524 (1991) 561600. doi : 10 .1016/0375-9474(91)90263-6.

[594] D. Diakonov, Chiral quark - soliton model, in: Nonperturbative quantum field physics. Proceedings, Advanced School, Peniscola, Spain, June 2-6, 1997, 1997, pp. 1-55. arXiv: hep-ph/9802298.

[595] H. Weigel, Chiral Soliton Models for Baryons, Lect. Notes Phys. 743 (2008) 1-274.

[596] Y.-L. Ma, M. Harada, Lecture notes on the Skyrme modelarXiv:1604.04850.

[597] E. Witten, Global Aspects of Current Algebra, Nucl. Phys. B223 (1983) 422-432. doi:10.1016/ 0550-3213(83) 90063-9.

[598] J. Wess, B. Zumino, Consequences of anomalous Ward identities, Phys. Lett. 37B (1971) 95-97. doi : 10.1016/0370-2693(71)90582-X.

[599] A. V. Manohar, Equivalence of the Chiral Soliton and Quark Models in Large N, Nucl. Phys. B248 (1984) 19, [,19(1984)]. doi:10.1016/0550-3213(84)90584-4. 
[600] M. Chemtob, Skyrme Model of Baryon Octet and Decuplet, Nucl. Phys. B256 (1985) 600-608. doi:10.1016/0550-3213(85)90409-2.

[601] P. O. Mazur, M. A. Nowak, M. Praszalowicz, SU(3) Extension of the Skyrme Model, Phys. Lett. 147B (1984) 137-140. doi:10.1016/0370-2693(84)90608-7.

[602] S. Jain, S. R. Wadia, Large $N$ Baryons: Collective Coordinates of the Topological Soliton in SU(3) Chiral Model, Nucl. Phys. B258 (1985) 713, [,713(1984)]. doi:10.1016/0550-3213(85)90632-7.

[603] M. Praszalowicz, in: Proceedings of the Workshop on Skyrmions and Anomalies, World Scientific, Singapore, 1987, p. 112.

[604] M. Praszalowicz, Pentaquark in the Skyrme model, Phys. Lett. B575 (2003) 234-241. arXiv: hep-ph/0308114, doi:10.1016/j.physletb.2003.09.049.

[605] J. Schechter, H. Weigel, The Skyrme model for baryons (1999) 337-369arXiv:hep-ph/9907554.

[606] E. Wuest, G. E. Brown, A. D. Jackson, Topological Chiral Bags in a Baryonic Environment, Nucl. Phys. A468 (1987) 450-472. doi:10.1016/0375-9474(87)90178-3.

[607] I. R. Klebanov, Nuclear Matter in the Skyrme Model, Nucl. Phys. B262 (1985) 133-143. doi: 10.1016/0550-3213(85)90068-9.

[608] H.-J. Lee, B.-Y. Park, D.-P. Min, M. Rho, V. Vento, A Unified approach to high density: Pion fluctuations in skyrmion matter, Nucl. Phys. A723 (2003) 427-446. arXiv:hep-ph/0302019, doi:10.1016/S0375-9474(03)01452-0.

[609] Y.-L. Ma, Y. Oh, G.-S. Yang, M. Harada, H. K. Lee, B.-Y. Park, M. Rho, Hidden Local Symmetry and Infinite Tower of Vector Mesons for Baryons, Phys. Rev. D86 (2012) 074025. arXiv:1206. 5460, doi:10.1103/PhysRevD.86.074025.

[610] Y.-L. Ma, G.-S. Yang, Y. Oh, M. Harada, Skyrmions with vector mesons in the hidden local symmetry approach, Phys. Rev. D87 (3) (2013) 034023. arXiv:1209.3554, doi:10.1103/PhysRevD. 87.034023.

[611] Y.-L. Ma, M. Harada, H. K. Lee, Y. Oh, B.-Y. Park, M. Rho, Dense baryonic matter in conformally-compensated hidden local symmetry: Vector manifestation and chiral symmetry restoration, Phys. Rev. D90 (3) (2014) 034015. arXiv:1308.6476, doi:10.1103/PhysRevD.90. 034015.

[612] Y.-L. Ma, M. Harada, H. K. Lee, Y. Oh, B.-Y. Park, M. Rho, Dense baryonic matter in the hidden local symmetry approach: Half-skyrmions and nucleon mass, Phys. Rev. D88 (1) (2013) 014016, [Erratum: Phys. Rev.D88,no.7,079904(2013)]. arXiv:1304.5638, doi:10.1103/PhysRevD.88. 014016, 10.1103/PhysRevD.88.079904.

[613] C. G. Callan, Jr., I. R. Klebanov, Bound State Approach to Strangeness in the Skyrme Model, Nucl. Phys. B262 (1985) 365-382. doi:10.1016/0550-3213(85)90292-5.

[614] M. A. Nowak, M. Rho, I. Zahed, Chiral effective action with heavy quark symmetry, Phys. Rev. D48 (1993) 4370-4374. arXiv:hep-ph/9209272, doi:10.1103/PhysRevD .48.4370.

[615] W. A. Bardeen, C. T. Hill, Chiral dynamics and heavy quark symmetry in a solvable toy field theoretic model, Phys. Rev. D49 (1994) 409-425. arXiv:hep-ph/9304265, doi:10.1103/PhysRevD. 49.409 . 
[616] M. A. Nowak, M. Praszalowicz, M. Sadzikowski, J. Wasiluk, Chiral doublers of heavy light baryons, Phys. Rev. D70 (2004) 031503. arXiv:hep-ph/0403184, doi:10.1103/PhysRevD.70. 031503.

[617] M. Harada, Y.-L. Ma, Chiral partner structure of heavy baryons from the bound state approach with hidden local symmetry, Phys. Rev. D87 (5) (2013) 056007. arXiv:1212.5079, doi:10. 1103/PhysRevD . 87.056007.

[618] D. Diakonov, V. Yu. Petrov, P. V. Pobylitsa, A Chiral Theory of Nucleons, Nucl. Phys. B306 (1988) 809. doi:10.1016/0550-3213(88)90443-9.

[619] C. Christov, A. Blotz, H.-C. Kim, P. Pobylitsa, T. Watabe, T. Meissner, E. Ruiz Arriola, K. Goeke, Baryons as nontopological chiral solitons, Prog. Part. Nucl. Phys. 37 (1996) 91-191. arXiv: hep-ph/9604441, doi:10.1016/0146-6410(96)00057-9.

[620] K. Goeke, H.-C. Kim, M. Praszalowicz, G.-S. Yang, Pentaquarks: Review on models and solitonic calculations of antidecuplet magnetic moments, Prog. Part. Nucl. Phys. 55 (2005) 350-373. arXiv: hep-ph/0411195, doi:10.1016/j.ppnp.2005.01.028.

[621] A. Blotz, K. Goke, N. W. Park, D. Diakonov, V. Petrov, P. V. Pobylitsa, Strange baryons in the solitonic sector of the Nambu-Jona-Lasinio model, Phys. Lett. B287 (1992) 29-34. doi: 10.1016/0370-2693(92) 91871-6.

[622] A. Blotz, D. Diakonov, K. Goeke, N. W. Park, V. Petrov, P. V. Pobylitsa, The SU(3) NambuJona-Lasinio soliton in the collective quantization formulation, Nucl. Phys. A555 (1993) 765-792. doi:10.1016/0375-9474(93)90505-R.

[623] G.-S. Yang, H.-C. Kim, New narrow nucleon resonances $N^{*}(1685)$ and $N^{*}(1726)$ within the chiral quark-soliton modelarXiv:1809.07489.

[624] R. L. Jaffe, The width of the Theta+ exotic baryon in the chiral soliton model, Eur. Phys. J. C35 (2004) 221-222. arXiv:hep-ph/0401187, doi:10.1140/epjc/s2004-01815-4.

[625] G.-S. Yang, H.-C. Kim, Mass splittings of SU(3) baryons within a chiral soliton model, Prog. Theor. Phys. 128 (2012) 397-413. arXiv:1010.3792, doi:10.1143/PTP.128.397.

[626] G.-S. Yang, H.-C. Kim, Mass splittings of the baryon decuplet and antidecuplet with the secondorder flavor symmetry breakings within a chiral soliton model, J. Korean Phys. Soc. 61 (2012) 1956-1964. arXiv:1102.1786, doi:10.3938/jkps.61.1956.

[627] V. Kuznetsov, et al., Evidence for a narrow structure at W 1.68-GeV in eta photoproduction on the neutron, Phys. Lett. B647 (2007) 23-29. arXiv:hep-ex/0606065, doi:10.1016/j .physletb. 2007.01 .041$.

[628] M. Praszalowicz, K. Goeke, Exotic eikosiheptaplet in the chiral quark soliton model, Phys. Rev. D76 (2007) 096003. doi:10.1103/PhysRevD.76.096003.

[629] M. Luscher, U. Wolff, How to Calculate the Elastic Scattering Matrix in Two-dimensional Quantum Field Theories by Numerical Simulation, Nucl. Phys. B339 (1990) 222-252. doi: 10.1016/0550-3213(90)90540-T.

[630] B. Blossier, M. Della Morte, G. von Hippel, T. Mendes, R. Sommer, On the generalized eigenvalue method for energies and matrix elements in lattice field theory, JHEP 04 (2009) 094. arXiv: 0902.1265, doi:10.1088/1126-6708/2009/04/094. 
[631] J. M. Bulava, et al., Excited State Nucleon Spectrum with Two Flavors of Dynamical Fermions, Phys. Rev. D79 (2009) 034505. arXiv:0901.0027, doi:10.1103/PhysRevD.79.034505.

[632] J. J. Dudek, R. G. Edwards, N. Mathur, D. G. Richards, Charmonium excited state spectrum in lattice QCD, Phys. Rev. D77 (2008) 034501. arXiv:0707.4162, doi:10.1103/PhysRevD.77. 034501.

[633] M. S. Mahbub, W. Kamleh, D. B. Leinweber, P. J. Moran, A. G. Williams, Structure and Flow of the Nucleon Eigenstates in Lattice QCD, Phys. Rev. D87 (9) (2013) 094506. arXiv:1302.2987, doi:10.1103/PhysRevD.87.094506.

[634] A. L. Kiratidis, W. Kamleh, D. B. Leinweber, Z.-W. Liu, F. M. Stokes, A. W. Thomas, Search for low-lying lattice QCD eigenstates in the Roper regime, Phys. Rev. D95 (7) (2017) 074507. arXiv:1608.03051, doi:10.1103/PhysRevD.95.074507.

[635] A. L. Kiratidis, W. Kamleh, D. B. Leinweber, B. J. Owen, Lattice baryon spectroscopy with multi-particle interpolators, Phys. Rev. D91 (2015) 094509. arXiv:1501.07667, doi:10.1103/ PhysRevD.91.094509.

[636] M. Luscher, Volume Dependence of the Energy Spectrum in Massive Quantum Field Theories. 2. Scattering States, Commun. Math. Phys. 105 (1986) 153-188. doi :10.1007/BF01211097.

[637] M. Luscher, Volume Dependence of the Energy Spectrum in Massive Quantum Field Theories. 1. Stable Particle States, Commun. Math. Phys. 104 (1986) 177. doi :10.1007/BF01211589.

[638] M. Luscher, Signatures of unstable particles in finite volume, Nucl. Phys. B364 (1991) 237-251. doi : 10.1016/0550-3213(91) 90584-K.

[639] M. Luscher, Two particle states on a torus and their relation to the scattering matrix, Nucl. Phys. B354 (1991) 531-578. doi:10.1016/0550-3213(91)90366-6.

[640] R. A. Briceno, J. J. Dudek, R. D. Young, Scattering processes and resonances from lattice QCD, Rev. Mod. Phys. 90 (2) (2018) 025001. arXiv:1706.06223, doi:10.1103/RevModPhys. 90.025001.

[641] M. Doring, U.-G. Meissner, E. Oset, A. Rusetsky, Unitarized Chiral Perturbation Theory in a finite volume: Scalar meson sector, Eur. Phys. J. A47 (2011) 139. arXiv:1107.3988, doi: 10.1140/epja/i2011-11139-7.

[642] M. Doring, J. Haidenbauer, U.-G. Meissner, A. Rusetsky, Dynamical coupled-channel approaches on a momentum lattice, Eur. Phys. J. A47 (2011) 163. arXiv:1108.0676, doi:10.1140/epja/ i2011-11163-7.

[643] T.-W. Chiu, T.-H. Hsieh, Y(4260) on the lattice, Phys. Rev. D73 (2006) 094510. arXiv:hep-lat/ 0512029, doi:10.1103/PhysRevD.73.094510.

[644] P. Lacock, C. Michael, P. Boyle, P. Rowland, Hybrid mesons from quenched QCD, Phys. Lett. B401 (1997) 308-312. arXiv:hep-lat/9611011, doi:10.1016/S0370-2693(97)00384-5.

[645] T. Manke, I. T. Drummond, R. R. Horgan, H. P. Shanahan, Heavy hybrids from NRQCD, Phys. Rev. D57 (1998) 3829-3832. arXiv:hep-lat/9710083, doi:10.1103/PhysRevD.57.3829. 
[646] K. J. Juge, J. Kuti, C. J. Morningstar, The Heavy hybrid spectrum from NRQCD and the Born-Oppenheimer approximation, Nucl. Phys. Proc. Suppl. 83 (2000) 304-306. arXiv: hep-lat/ 9909165, doi:10.1016/S0920-5632(00)91655-4.

[647] I. T. Drummond, N. A. Goodman, R. R. Horgan, H. P. Shanahan, L. C. Storoni, Spin effects in heavy hybrid mesons on an anisotropic lattice, Phys. Lett. B478 (2000) 151-160. arXiv: hep-lat/9912041, doi:10.1016/S0370-2693(00)00225-2.

[648] L. Liu, G. Moir, M. Peardon, S. M. Ryan, C. E. Thomas, P. Vilaseca, J. J. Dudek, R. G. Edwards, B. Joo, D. G. Richards, Excited and exotic charmonium spectroscopy from lattice QCD, JHEP 07 (2012) 126. arXiv:1204.5425, doi:10.1007/JHEP07(2012) 126.

[649] X.-Q. Luo, Y. Liu, Gluonic excitation of non-exotic hybrid charmonium from lattice QCD, Phys. Rev. D74 (2006) 034502, [Erratum: Phys. Rev.D74,039902(2006)]. arXiv:hep-lat/0512044, doi:10.1103/PhysRevD.74.034502,10.1103/PhysRevD.74.039902.

[650] W. Chen, R. T. Kleiv, T. G. Steele, B. Bulthuis, D. Harnett, J. Ho, T. Richards, S.-L. Zhu, Mass Spectrum of Heavy Quarkonium Hybrids, JHEP 09 (2013) 019. arXiv:1304.4522, doi: 10.1007/JHEP09 (2013) 019.

[651] W. Chen, T. G. Steele, S.-L. Zhu, Masses of the bottom-charm hybrid $\bar{b} G c$ states, J. Phys. G41 (2014) 025003. arXiv:1306.3486, doi:10.1088/0954-3899/41/2/025003.

[652] F.-K. Guo, U.-G. Meissner, Light quark mass dependence in heavy quarkonium physics, Phys. Rev. Lett. 109 (2012) 062001. arXiv:1203.1116, doi:10.1103/PhysRevLett.109.062001.

[653] G. K. C. Cheung, C. O’Hara, G. Moir, M. Peardon, S. M. Ryan, C. E. Thomas, D. Tims, Excited and exotic charmonium, $D_{s}$ and $D$ meson spectra for two light quark masses from lattice QCD, JHEP 12 (2016) 089. arXiv:1610.01073, doi:10.1007/JHEP12(2016)089.

[654] J. J. Dudek, R. G. Edwards, M. J. Peardon, D. G. Richards, C. E. Thomas, Toward the excited meson spectrum of dynamical QCD, Phys. Rev. D82 (2010) 034508. arXiv:1004.4930, doi: 10.1103/PhysRevD.82.034508.

[655] G. Moir, M. Peardon, S. M. Ryan, C. E. Thomas, L. Liu, Excited spectroscopy of charmed mesons from lattice QCD, JHEP 05 (2013) 021. arXiv:1301.7670, doi:10.1007/JHEP05(2013) 021.

[656] G. Moir, M. Peardon, S. M. Ryan, C. E. Thomas, D. J. Wilson, Coupled-Channel $D \pi, D \eta$ and $D_{s} \bar{K}$ Scattering from Lattice QCD, JHEP 10 (2016) 011. arXiv:1607.07093, doi:10.1007/ JHEP10 (2016) 011.

[657] Y. Chen, W.-F. Chiu, M. Gong, L.-C. Gui, Z. Liu, Exotic vector charmonium and its leptonic decay width, Chin. Phys. C40 (8) (2016) 081002. arXiv:1604.03401, doi:10.1088/1674-1137/ 40/8/081002.

[658] C. J. Morningstar, M. J. Peardon, Efficient glueball simulations on anisotropic lattices, Phys. Rev. D56 (1997) 4043-4061. arXiv:hep-lat/9704011, doi:10.1103/PhysRevD.56.4043.

[659] C. J. Morningstar, M. J. Peardon, The Glueball spectrum from an anisotropic lattice study, Phys. Rev. D60 (1999) 034509. arXiv:hep-lat/9901004, doi:10.1103/PhysRevD.60.034509.

[660] Y. Chen, et al., Glueball spectrum and matrix elements on anisotropic lattices, Phys. Rev. D73 (2006) 014516. arXiv:hep-lat/0510074, doi:10.1103/PhysRevD.73.014516. 
[661] T.-W. Chiu, T.-H. Hsieh, X(3872) in lattice QCD with exact chiral symmetry, Phys. Lett. B646 (2007) 95-99. arXiv:hep-ph/0603207, doi:10.1016/j.physletb.2007.01.019.

[662] T.-W. Chiu, T.-H. Hsieh, Pseudovector meson with strangeness and closed-charm, Phys. Rev. D73 (2006) 111503, [Erratum: Phys. Rev. D75, 019902 (2007)]. arXiv:hep-lat/0604008, doi: 10.1103/PhysRevD.73.111503,10.1103/PhysRevD.75.019902.

[663] S. Prelovsek, T. Draper, C. B. Lang, M. Limmer, K.-F. Liu, N. Mathur, D. Mohler, Lattice study of light scalar tetraquarks with $I=0,2,1 / 2,3 / 2$ : Are $\sigma$ and $\kappa$ tetraquarks?, Phys. Rev. D82 (2010) 094507. arXiv:1005.0948, doi:10.1103/PhysRevD.82.094507.

[664] F.-K. Guo, L. Liu, U.-G. Meissner, P. Wang, Tetraquarks, hadronic molecules, meson-meson scattering and disconnected contributions in lattice QCD, Phys. Rev. D88 (2013) 074506. arXiv: 1308.2545, doi:10.1103/PhysRevD.88.074506.

[665] G. S. Bali, H. Neff, T. Duessel, T. Lippert, K. Schilling, Observation of string breaking in QCD, Phys. Rev. D71 (2005) 114513. arXiv:hep-lat/0505012, doi:10.1103/PhysRevD.71.114513.

[666] G. Bali, et al., Spectra of heavy-light and heavy-heavy mesons containing charm quarks, including higher spin states for $N_{f}=2+1$, PoS LATTICE2011 (2011) 135. arXiv:1108.6147, doi: $10.22323 / 1.139 .0135$.

[667] G. Bali, S. Collins, P. Perez-Rubio, Charmed hadron spectroscopy on the lattice for $N_{f}=2+1$ flavours, J. Phys. Conf. Ser. 426 (2013) 012017. arXiv:1212.0565, doi:10.1088/1742-6596/ $426 / 1 / 012017$.

[668] D. Mohler, S. Prelovsek, R. M. Woloshyn, $D \pi$ scattering and $D$ meson resonances from lattice QCD, Phys. Rev. D87 (3) (2013) 034501. arXiv:1208.4059, doi:10.1103/PhysRevD.87.034501.

[669] G. S. Bali, S. Collins, C. Ehmann, Charmonium spectroscopy and mixing with light quark and open charm states from $n_{F}=2$ lattice QCD, Phys. Rev. D84 (2011) 094506. arXiv:1110.2381, doi:10.1103/PhysRevD.84.094506.

[670] M. Suzuki, The X(3872) boson: Molecule or charmonium, Phys. Rev. D72 (2005) 114013. arXiv: hep-ph/0508258, doi:10.1103/PhysRevD.72.114013.

[671] C. Meng, Y.-J. Gao, K.-T. Chao, $B \rightarrow \chi_{c 1}(1 P, 2 P) K$ decays in QCD factorization and $X(3872)$, Phys. Rev. D87 (7) (2013) 074035. arXiv:hep-ph/0506222, doi:10.1103/PhysRevD .87.074035.

[672] S. Prelovsek, L. Leskovec, Evidence for X(3872) from $D D^{*}$ scattering on the lattice, Phys. Rev. Lett. 111 (2013) 192001. arXiv:1307.5172, doi:10.1103/PhysRevLett.111.192001.

[673] S.-h. Lee, C. DeTar, H. Na, D. Mohler, Searching for the $X(3872)$ and $Z_{c}^{+}(3900)$ on HISQ LatticesarXiv:1411.1389.

[674] M. Padmanath, C. B. Lang, S. Prelovsek, X(3872) and Y(4140) using diquark-antidiquark operators with lattice QCD, Phys. Rev. D92 (3) (2015) 034501. arXiv:1503.03257, doi: 10.1103/PhysRevD.92.034501.

[675] V. Baru, E. Epelbaum, A. A. Filin, C. Hanhart, U. G. Meissner, A. V. Nefediev, Quark mass dependence of the $X(3872)$ binding energy, Phys. Lett. B726 (2013) 537-543. arXiv:1306.4108, doi:10.1016/j.physletb.2013.08.073. 
[676] M. Jansen, H. W. Hammer, Y. Jia, Light quark mass dependence of the $X(3872)$ in an effective field theory, Phys. Rev. D89 (1) (2014) 014033. arXiv:1310.6937, doi:10.1103/PhysRevD.89. 014033.

[677] E. J. Garzon, R. Molina, A. Hosaka, E. Oset, Strategies for an accurate determination of the X(3872) energy from QCD lattice simulations, Phys. Rev. D89 (2014) 014504. arXiv:1310.0972, doi:10.1103/PhysRevD.89.014504.

[678] M. Albaladejo, C. Hidalgo-Duque, J. Nieves, E. Oset, Hidden charm molecules in finite volume, Phys. Rev. D88 (2013) 014510. arXiv:1304.1439, doi:10.1103/PhysRevD.88.014510.

[679] N. Li, S.-L. Zhu, Isospin breaking, Coupled-channel effects and Diagnosis of X(3872), Phys. Rev. D86 (2012) 074022. arXiv:1207.3954, doi:10.1103/PhysRevD.86.074022.

[680] L. Zhao, L. Ma, S.-L. Zhu, Spin-orbit force, recoil corrections, and possible $B \bar{B}^{*}$ and $D \bar{D}^{*}$ molecular states, Phys. Rev. D89 (9) (2014) 094026. arXiv:1403.4043, doi:10.1103/PhysRevD.89. 094026.

[681] X. Liu, Z.-G. Luo, Y.-R. Liu, S.-L. Zhu, X(3872) and Other Possible Heavy Molecular States, Eur. Phys. J. C61 (2009) 411-428. arXiv:0808.0073, doi:10.1140/epjc/s10052-009-1020-4.

[682] Z.-F. Sun, Z.-G. Luo, J. He, X. Liu, S.-L. Zhu, A note on the $B^{*} \bar{B}, B^{*} \bar{B}^{*}, D^{*} \bar{D}, D^{*} \bar{D}^{*}$ molecular states, Chin. Phys. C36 (2012) 194-204. doi:10.1088/1674-1137/36/3/002.

[683] J. He, Study of the $B \bar{B}^{*} / D \bar{D}^{*}$ bound states in a Bethe-Salpeter approach, Phys. Rev. D90 (7) (2014) 076008. arXiv:1409.8506, doi:10.1103/PhysRevD.90.076008.

[684] S. Prelovsek, L. Leskovec, Search for $Z_{c}^{+}(3900)$ in the $1^{+-}$Channel on the Lattice, Phys. Lett. B727 (2013) 172-176. arXiv:1308.2097, doi:10.1016/j.physletb.2013.10.009.

[685] L. Levkova, C. DeTar, Charm annihilation effects on the hyperfine splitting in charmonium, Phys. Rev. D83 (2011) 074504. arXiv:1012.1837, doi:10.1103/PhysRevD.83.074504.

[686] S. Prelovsek, C. B. Lang, L. Leskovec, D. Mohler, Study of the $Z_{c}^{+}$channel using lattice QCD, Phys. Rev. D91 (1) (2015) 014504. arXiv:1405.7623, doi:10.1103/PhysRevD.91.014504.

[687] D.-Y. Chen, X. Liu, T. Matsuki, Reproducing the $Z_{c}(3900)$ structure through the initial-singlepion-emission mechanism, Phys. Rev. D88 (3) (2013) 036008. arXiv:1304.5845, doi:10.1103/ PhysRevD .88.036008.

[688] E. Swanson, $Z_{b}$ and $Z_{c}$ Exotic States as Coupled Channel Cusps, Phys. Rev. D91 (3) (2015) 034009. arXiv:1409.3291, doi:10.1103/PhysRevD.91.034009.

[689] A. L. Guerrieri, M. Papinutto, A. Pilloni, A. D. Polosa, N. Tantalo, Flavored tetraquark spectroscopy, PoS LATTICE2014 (2015) 106. arXiv:1411.2247, doi:10.22323/1.214.0106.

[690] Y. Chen, et al., Low-energy scattering of the $\left(D \bar{D}^{*}\right)^{ \pm}$system and the resonance-like structure $Z_{c}(3900)$, Phys. Rev. D89 (9) (2014) 094506. arXiv:1403.1318, doi:10.1103/PhysRevD.89. 094506.

[691] Y. Chen, et al., Low-energy Scattering of $\left(D^{*} \bar{D}^{*}\right)^{ \pm}$System and the Resonance-like Structure $Z_{c}$ (4025), Phys. Rev. D92 (5) (2015) 054507. arXiv:1503.02371, doi:10.1103/PhysRevD.92. 054507. 
[692] Y. Ikeda, S. Aoki, T. Doi, S. Gongyo, T. Hatsuda, T. Inoue, T. Iritani, N. Ishii, K. Murano, K. Sasaki, Fate of the Tetraquark Candidate $Z_{c}(3900)$ from Lattice QCD, Phys. Rev. Lett. 117 (24) (2016) 242001. arXiv:1602.03465, doi:10.1103/PhysRevLett.117.242001.

[693] Y. Ikeda, The tetraquark candidate $Z_{c}(3900)$ from dynamical lattice QCD simulations, J. Phys. G45 (2) (2018) 024002. arXiv:1706.07300, doi:10.1088/1361-6471/aa9afd.

[694] S. Aoki, N. Ishii, T. Doi, T. Hatsuda, Y. Ikeda, T. Inoue, K. Murano, H. Nemura, K. Sasaki, Extraction of Hadron Interactions above Inelastic Threshold in Lattice QCD, Proc. Japan Acad. B87 (2011) 509-517. arXiv:1106.2281, doi:10.2183/pjab.87.509.

[695] S. Aoki, B. Charron, T. Doi, T. Hatsuda, T. Inoue, N. Ishii, Construction of energy-independent potentials above inelastic thresholds in quantum field theories, Phys. Rev. D87 (3) (2013) 034512. arXiv:1212.4896, doi:10.1103/PhysRevD.87.034512.

[696] K. Sasaki, S. Aoki, T. Doi, T. Hatsuda, Y. Ikeda, T. Inoue, N. Ishii, K. Murano, Coupled-channel approach to strangeness $S=-2$ baryonCbayron interactions in lattice QCD, PTEP 2015 (11) (2015) 113B01. arXiv:1504.01717, doi:10.1093/ptep/ptv144.

[697] G. K. C. Cheung, C. E. Thomas, J. J. Dudek, R. G. Edwards, Tetraquark operators in lattice QCD and exotic flavour states in the charm sector, JHEP 11 (2017) 033. arXiv:1709.01417, doi:10.1007/JHEP11(2017)033.

[698] M. Peardon, J. Bulava, J. Foley, C. Morningstar, J. Dudek, R. G. Edwards, B. Joo, H.-W. Lin, D. G. Richards, K. J. Juge, A Novel quark-field creation operator construction for hadronic physics in lattice QCD, Phys. Rev. D80 (2009) 054506. arXiv:0905.2160, doi:10.1103/PhysRevD.80. 054506.

[699] R. G. Edwards, B. Joo, H.-W. Lin, Tuning for Three-flavors of Anisotropic Clover Fermions with Stout-link Smearing, Phys. Rev. D78 (2008) 054501. arXiv:0803.3960, doi:10.1103/PhysRevD. 78.054501.

[700] G.-Z. Meng, et al., Low-energy $D^{*+} D_{1}^{0}$ Scattering and the Resonance-like Structure $Z^{+}(4430)$, Phys. Rev. D80 (2009) 034503. arXiv:0905.0752, doi:10.1103/PhysRevD.80.034503.

[701] T. Chen, et al., A Lattice Study of $\left(\bar{D}_{1} D^{*}\right)^{ \pm}$Near-threshold Scattering, Phys. Rev. D93 (11) (2016) 114501. arXiv:1602.00200, doi:10.1103/PhysRevD.93.114501.

[702] D. G. Richards, D. K. Sinclair, D. W. Sivers, Lattice QCD simulation of meson exchange forces, Phys. Rev. D42 (1990) 3191-3196. doi:10.1103/PhysRevD.42.3191.

[703] A. Mihaly, H. R. Fiebig, H. Markum, K. Rabitsch, Interactions between heavy - light mesons in lattice QCD, Phys. Rev. D55 (1997) 3077-3081. doi:10.1103/PhysRevD.55.3077.

[704] P. Pennanen, C. Michael, A. M. Green, Interactions of heavy light mesons, Nucl. Phys. Proc. Suppl. 83 (2000) 200-202. arXiv:hep-lat/9908032, doi:10.1016/S0920-5632(00)91622-0.

[705] A. M. Green, J. Koponen, P. Pennanen, A Variational fit to the lattice energy of two heavy light mesons, Phys. Rev. D61 (2000) 014014. arXiv:hep-ph/9902249, doi:10.1103/PhysRevD.61. 014014.

[706] J. M. Richard, Tetraquarks, pentaquarks and hexaquarks, Nucl. Phys. Proc. Suppl. 21 (1991) 254-257. doi:10.1016/0920-5632(91)90263-E. 
[707] M. Bander, A. Subbaraman, Baryons with two heavy quarks as solitons, Phys. Rev. D50 (1994) R5478-R5480. arXiv:hep-ph/9407309, doi:10.1103/PhysRevD.50.R5478.

[708] W. Detmold, K. Orginos, M. J. Savage, BB Potentials in Quenched Lattice QCD, Phys. Rev. D76 (2007) 114503. arXiv:hep-lat/0703009, doi:10.1103/PhysRevD .76.114503.

[709] G. Bali, M. Hetzenegger, Static-light meson-meson potentials, PoS LATTICE2010 (2010) 142. arXiv:1011.0571, doi:10.22323/1.105.0142.

[710] Z. S. Brown, K. Orginos, Tetraquark bound states in the heavy-light heavy-light system, Phys. Rev. D86 (2012) 114506. arXiv:1210.1953, doi:10.1103/PhysRevD.86.114506.

[711] M. Wagner, Forces between static-light mesons, PoS LATTICE2010 (2010) 162. arXiv:1008. 1538, doi:10.22323/1.105.0162.

[712] M. Wagner, Static-static-light-light tetraquarks in lattice QCD, Acta Phys. Polon. Supp. 4 (2011) 747-752. arXiv:1103.5147, doi:10.5506/APhysPolBSupp.4.747.

[713] P. Bicudo, J. Scheunert, M. Wagner, Including heavy spin effects in the prediction of a $\bar{b} \bar{b} u d$ tetraquark with lattice QCD potentials, Phys. Rev. D95 (3) (2017) 034502. arXiv:1612.02758, doi:10.1103/PhysRevD.95.034502.

[714] A. Francis, R. J. Hudspith, R. Lewis, K. Maltman, Lattice Prediction for Deeply Bound Doubly Heavy Tetraquarks, Phys. Rev. Lett. 118 (14) (2017) 142001. arXiv:1607.05214, doi:10.1103/ PhysRevLett.118.142001.

[715] A. V. Manohar, The HQET / NRQCD Lagrangian to order alpha / m-3, Phys. Rev. D56 (1997) 230-237. arXiv:hep-ph/9701294, doi:10.1103/PhysRevD.56.230.

[716] G. P. Lepage, L. Magnea, C. Nakhleh, U. Magnea, K. Hornbostel, Improved nonrelativistic QCD for heavy quark physics, Phys. Rev. D46 (1992) 4052-4067. arXiv:hep-lat/9205007, doi: 10.1103/PhysRevD.46.4052.

[717] B. A. Thacker, G. P. Lepage, Heavy quark bound states in lattice QCD, Phys. Rev. D43 (1991) 196-208. doi:10.1103/PhysRevD.43.196.

[718] A. Francis, R. J. Hudspith, R. Lewis, K. Maltman, Evidence for charm-bottom tetraquarks and the mass dependence of heavy-light tetraquark states from lattice QCD, Phys. Rev. D99 (5) (2019) 054505. arXiv:1810.10550, doi:10.1103/PhysRevD.99.054505.

[719] Y. Ikeda, B. Charron, S. Aoki, T. Doi, T. Hatsuda, T. Inoue, N. Ishii, K. Murano, H. Nemura, K. Sasaki, Charmed tetraquarks $T_{c c}$ and $T_{c s}$ from dynamical lattice QCD simulations, Phys. Lett. B729 (2014) 85-90. arXiv:1311.6214, doi:10.1016/j.physletb.2014.01.002.

[720] P. Junnarkar, N. Mathur, M. Padmanath, Study of doubly heavy tetraquarks in Lattice QCD, Phys. Rev. D99 (2019) 034507. arXiv:1810.12285, doi:10.1103/PhysRevD.99.034507.

[721] H. Neuberger, Exactly massless quarks on the lattice, Phys. Lett. B417 (1998) 141-144. arXiv: hep-lat/9707022, doi:10.1016/S0370-2693(97)01368-3.

[722] H. Neuberger, More about exactly massless quarks on the lattice, Phys. Lett. B427 (1998) 353-355. arXiv:hep-lat/9801031, doi:10.1016/S0370-2693(98)00355-4.

[723] C. B. Lang, D. Mohler, S. Prelovsek, $B_{s} \pi^{+}$scattering and search for X(5568) with lattice QCD, Phys. Rev. D94 (2016) 074509. arXiv:1607.03185, doi:10.1103/PhysRevD.94.074509. 
[724] C. B. Lang, L. Leskovec, D. Mohler, S. Prelovsek, R. M. Woloshyn, Ds mesons with DK and D*K scattering near threshold, Phys. Rev. D90 (3) (2014) 034510. arXiv:1403.8103, doi: 10.1103/PhysRevD.90.034510.

[725] A. X. El-Khadra, A. S. Kronfeld, P. B. Mackenzie, Massive fermions in lattice gauge theory, Phys. Rev. D55 (1997) 3933-3957. arXiv:hep-lat/9604004, doi:10.1103/PhysRevD.55.3933.

[726] M. B. Oktay, A. S. Kronfeld, New lattice action for heavy quarks, Phys. Rev. D78 (2008) 014504. arXiv:0803.0523, doi:10.1103/PhysRevD.78.014504.

[727] C. Hughes, E. Eichten, C. T. H. Davies, Searching for beauty-fully bound tetraquarks using lattice nonrelativistic QCD, Phys. Rev. D97 (5) (2018) 054505. arXiv:1710.03236, doi:10. 1103/PhysRevD.97.054505.

[728] T. Kawanai, S. Sasaki, Charmonium-nucleon potential from lattice QCD, Phys. Rev. D82 (2010) 091501. arXiv:1009.3332, doi:10.1103/PhysRevD.82.091501.

[729] M. Luscher, S. Sint, R. Sommer, P. Weisz, U. Wolff, Nonperturbative O(a) improvement of lattice QCD, Nucl. Phys. B491 (1997) 323-343. arXiv:hep-lat/9609035, doi:10.1016/ S0550-3213(97)00080-1.

[730] S. Aoki, Y. Kuramashi, S.-i. Tominaga, Relativistic heavy quarks on the lattice, Prog. Theor. Phys. 109 (2003) 383-413. arXiv:hep-lat/0107009, doi:10.1143/PTP.109.383.

[731] K. Yokokawa, S. Sasaki, T. Hatsuda, A. Hayashigaki, First lattice study of low-energy charmonium-hadron interaction, Phys. Rev. D74 (2006) 034504. arXiv:hep-lat/0605009, doi:10.1103/PhysRevD.74.034504.

[732] L. Liu, H.-W. Lin, K. Orginos, Charmed Hadron Interactions, PoS LATTICE2008 (2008) 112. arXiv:0810.5412, doi:10.22323/1.066.0112.

[733] S. R. Beane, E. Chang, S. D. Cohen, W. Detmold, H. W. Lin, K. Orginos, A. Parreño, M. J. Savage, Quarkonium-Nucleus Bound States from Lattice QCD, Phys. Rev. D91 (11) (2015) 114503. arXiv: 1410.7069, doi:10.1103/PhysRevD.91.114503.

[734] T. Sugiura, Y. Ikeda, N. Ishii, Charmonium-nucleon interactions from the time-dependent HAL QCD method, EPJ Web Conf. 175 (2018) 05011. arXiv:1711.11219, doi:10.1051/epjconf/ 201817505011.

[735] U. Skerbis, S. Prelovsek, Nucleon- $J / \psi$ and nucleon- $\eta_{c}$ scattering in $P_{c}$ pentaquark channels from LQCDarXiv: 1811.02285.

[736] S. Dubynskiy, M. B. Voloshin, Hadro-Charmonium, Phys. Lett. B666 (2008) 344-346. arXiv: 0803.2224, doi:10.1016/j.physletb.2008.07.086.

[737] M. Alberti, G. S. Bali, S. Collins, F. Knechtli, G. Moir, W. Söldner, Hadroquarkonium from lattice QCD, Phys. Rev. D95 (7) (2017) 074501. arXiv:1608.06537, doi:10.1103/PhysRevD. 95.074501 .

[738] J. P. Ma, Z. G. Si, Factorization approach for inclusive production of doubly heavy baryon, Phys. Lett. B568 (2003) 135-145. arXiv:hep-ph/0305079, doi:10.1016/j.physletb.2003.06.064.

[739] Y. Jin, S.-Y. Li, Y.-R. Liu, Z.-G. Si, T. Yao, Search for a doubly charmed hadron at B factories, Phys. Rev. D89 (9) (2014) 094006. arXiv:1401.6652, doi:10.1103/PhysRevD.89.094006. 
[740] S.-Y. Li, Y.-R. Liu, Y.-N. Liu, Z.-G. Si, X.-F. Zhang, Hidden-charm Pentaquark Production at $e^{+} e^{-}$Colliders, Commun. Theor. Phys. 69 (3) (2018) 291. arXiv:1706.04765, doi:10.1088/ 0253-6102/69/3/291.

[741] W. Han, S.-Y. Li, Y.-H. Shang, F.-L. Shao, T. Yao, Exotic hadron production in quark combination model, Phys. Rev. C80 (2009) 035202. arXiv:0906.2473, doi:10.1103/PhysRevC.80. 035202.

[742] S. Cho, et al., Multi-quark hadrons from Heavy Ion Collisions, Phys. Rev. Lett. 106 (2011) 212001. arXiv:1011.0852, doi:10.1103/PhysRevLett.106.212001.

[743] S. Cho, et al., Studying Exotic Hadrons in Heavy Ion Collisions, Phys. Rev. C84 (2011) 064910. arXiv:1107.1302, doi:10.1103/PhysRevC.84.064910.

[744] S. Cho, et al., Exotic Hadrons from Heavy Ion Collisions, Prog. Part. Nucl. Phys. 95 (2017) 279-322. arXiv:1702.00486, doi:10.1016/j.ppnp.2017.02.002.

[745] X. Liu, H.-W. Ke, X. Liu, X.-Q. Li, Exploring open-charm decay mode $\Lambda_{c} \bar{\Lambda}_{c}$ of charmonium-like state $Y(4630)$, arXiv:1601.00762.

[746] L.-Y. Xiao, X.-Z. Weng, Q.-F. L, X.-H. Zhong, S.-L. Zhu, A new decay mode of higher charmonium, Eur. Phys. J. C78 (7) (2018) 605. arXiv:1805.07096, doi:10.1140/epjc/ s10052-018-6087-3.

[747] E. S. Ackleh, T. Barnes, Two photon widths of singlet positronium and quarkonium with arbitrary total angular momentum, Phys. Rev. D45 (1992) 232-240. doi:10.1103/PhysRevD.45.232.

[748] C.-Y. Wong, E. S. Swanson, T. Barnes, Heavy quarkonium dissociation cross-sections in relativistic heavy ion collisions, Phys. Rev. C65 (2002) 014903, [Erratum: Phys. Rev.C66,029901(2002)]. arXiv:nucl-th/0106067, doi:10.1103/PhysRevC.66.029901,10.1103/PhysRevC.65.014903.

[749] T. Barnes, E. S. Swanson, C. Y. Wong, X. M. Xu, Dissociation cross-sections of ground state and excited charmonia with light mesons in the quark model, Phys. Rev. C68 (2003) 014903. arXiv:nucl-th/0302052, doi:10.1103/PhysRevC.68.014903.

[750] G.-J. Wang, X.-H. Liu, L. Ma, X. Liu, X.-L. Chen, W.-Z. Deng, S.-L. Zhu, The strong decay patterns of $Z_{c}$ and $Z_{b}$ states in the relativized quark modelarXiv:1811.10339.

[751] X.-D. Guo, D.-Y. Chen, H.-W. Ke, X. Liu, X.-Q. Li, Study on the rare decays of Y(4630) induced by final state interactions, Phys. Rev. D93 (5) (2016) 054009. arXiv:1602.02222, doi:10.1103/ PhysRevD.93.054009.

[752] Z.-Y. Zhou, Z. Xiao, H.-Q. Zhou, Could the X(3915) and the X(3930) Be the Same Tensor State?, Phys. Rev. Lett. 115 (2) (2015) 022001. arXiv:1501.00879, doi:10.1103/PhysRevLett.115. 022001.

[753] V. Baru, C. Hanhart, A. V. Nefediev, Can X(3915) be the tensor partner of the X(3872)?, JHEP 06 (2017) 010. arXiv:1703.01230, doi:10.1007/JHEP06(2017)010.

[754] M. Albaladejo, F.-K. Guo, C. Hanhart, U.-G. Meissner, J. Nieves, A. Nogga, Z. Yang, Note on $\mathrm{X}(3872)$ production at hadron colliders and its molecular structure, Chin. Phys. C41 (12) (2017) 121001. arXiv:1709.09101, doi:10.1088/1674-1137/41/12/121001. 
[755] S. Cho, S. H. Lee, Hadronic effects on the X(3872) meson abundance in heavy ion collisions, Phys. Rev. C88 (2013) 054901. arXiv:1302.6381, doi:10.1103/PhysRevC.88.054901.

[756] F. Carvalho, E. R. Cazaroto, V. P. Gonçalves, F. S. Navarra, Tetraquark Production in Double Parton Scattering, Phys. Rev. D93 (3) (2016) 034004, [Phys. Rev.D93,034004(2016)]. arXiv: 1511.05209, doi:10.1103/PhysRevD.93.034004.

[757] W. Wang, Q. Zhao, Decipher the short-distance component of $X(3872)$ in $B_{c}$ decays, Phys. Lett. B755 (2016) 261-264. arXiv:1512.03123, doi:10.1016/j.physletb.2016.02.012.

[758] Y. K. Hsiao, C. Q. Geng, Search for $X Y Z$ states in $\Lambda_{b}$ decays at the LHCb, Phys. Lett. B757 (2016) 47-49. arXiv:1602.01236, doi:10.1016/j.physletb.2016.03.055.

[759] Y. K. Hsiao, C. Q. Geng, Branching fractions of $B_{(c)}$ decays involving $J / \psi$ and $X(3872)$, Chin. Phys. C41 (1) (2017) 013101. arXiv:1607.02718, doi:10.1088/1674-1137/41/1/013101.

[760] Z.-H. Wang, Y. Zhang, T.-h. Wang, Y. Jiang, G.-L. Wang, The Production of X(3940) and $X(4160)$ in $B_{c}$ decays, J. Phys. G43 (10) (2016) 105002. arXiv:1605.09091, doi:10.1088/ 0954-3899/43/10/105002.

[761] Z.-H. Wang, Y. Zhang, L. Jiang, T.-H. Wang, Y. Jiang, G.-L. Wang, The Strong Decays of X(3940) and X(4160), Eur. Phys. J. C77 (1) (2017) 43. arXiv:1608.05201, doi:10.1140/epjc/ s10052-017-4596-0.

[762] P. del Amo Sanchez, et al., Evidence for the decay $X(3872) \rightarrow J / \psi \omega$, Phys. Rev. D82 (2010) 011101. arXiv:1005.5190, doi:10.1103/PhysRevD.82.011101.

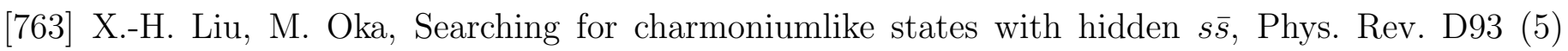
(2016) 054032. arXiv:1512.05474, doi:10.1103/PhysRevD.93.054032.

[764] L. M. Abreu, Analysis of X(3872) production via Heavy-Meson Effective Theory, PTEP 2016 (10) (2016) 103B01. arXiv:1608.08165, doi:10.1093/ptep/ptw138.

[765] L. M. Abreu, K. P. Khemchandani, A. Martinez Torres, F. S. Navarra, M. Nielsen, X(3872) production and absorption in a hot hadron gas, Phys. Lett. B761 (2016) 303-309. arXiv:1604. 07716, doi:10.1016/j.physletb.2016.08.050.

[766] L. M. Abreu, A. Lafayette Vasconcellos, Production of $Z_{b}^{(')}$ states in heavy-meson effective theory, Phys. Rev. D94 (9) (2016) 096009. doi:10.1103/PhysRevD.94.096009.

[767] L. M. Abreu, K. P. Khemchandani, A. Martnez Torres, F. S. Navarra, M. Nielsen, A. L. Vasconcellos, Production and absorption of exotic bottomoniumlike states in high energy heavy ion collisions, Phys. Rev. D95 (9) (2017) 096002. arXiv:1704.08781, doi:10.1103/PhysRevD.95. 096002.

[768] L. M. Abreu, F. S. Navarra, M. Nielsen, A. L. Vasconcellos, $Z_{b}(10610)$ in a hadronic medium, Eur. Phys. J. C78 (9) (2018) 752. arXiv:1711.05205, doi:10.1140/epjc/s10052-018-6182-5.

[769] Y. Jin, S.-Y. Li, Y.-R. Liu, L. Meng, Z.-G. Si, X.-F. Zhang, Exotic hadron bound state production at hadron colliders, Chin. Phys. C41 (8) (2017) 083106. arXiv:1610.04411, doi: 10.1088/1674-1137/41/8/083106.

[770] D.-Y. Chen, Where are $\chi_{c J}(3 P)$ ?, Eur. Phys. J. C76 (12) (2016) 671. arXiv:1611.00109, doi:10.1140/epjc/s10052-016-4531-9. 
[771] X.-W. Kang, J. A. Oller, Different pole structures in line shapes of the $X(3872)$, Eur. Phys. J. C77 (6) (2017) 399. arXiv:1612.08420, doi:10.1140/epjc/s10052-017-4961-z.

[772] E. Wang, J.-J. Xie, L.-S. Geng, E. Oset, Analysis of the $B^{+} \rightarrow J / \psi \phi K^{+}$data at low $J / \psi \phi$ invariant masses and the $X(4140)$ and $X(4160)$ resonances, Phys. Rev. D97 (1) (2018) 014017. arXiv:1710.02061, doi:10.1103/PhysRevD.97.014017.

[773] L. R. Dai, J. M. Dias, E. Oset, Disclosing $D^{*} \bar{D}^{*}$ molecular states in the $B_{c}^{-} \rightarrow \pi^{-} J / \psi \omega$ decay, Eur. Phys. J. C78 (3) (2018) 210. arXiv:1801.07091, doi:10.1140/epjc/s10052-018-5702-7.

[774] N. Ikeno, M. Bayar, E. Oset, Semileptonic decay of $B_{c}^{-}$into $X(3930), X(3940), X(4160)$, Eur. Phys. J. C78 (5) (2018) 429. arXiv:1803.11226, doi:10.1140/epjc/s10052-018-5898-6.

[775] V. P. Goncalves, B. D. Moreira, Probing the X(4350) in $\gamma \gamma$ interactions at the LHC, Eur. Phys. J. C79 (1) (2019) 7. arXiv:1809.08125, doi:10.1140/epjc/s10052-018-6517-2.

[776] E. Braaten, L.-P. He, K. Ingles, Predictive Solution to the X(3872) Collider Production PuzzlearXiv: 1811.08876.

[777] E. Braaten, L.-P. He, K. Ingles, Production of X(3872) Accompanied by a Pion at Hadron CollidersarXiv: 1903.04355.

[778] E. Braaten, L.-P. He, K. Ingles, Production of $X(3872)$ Accompanied by a Pion in $B$ Meson DecayarXiv: 1902.03259.

[779] A. Andronic, P. Braun-Munzinger, M. K. Köhler, K. Redlich, J. Stachel, Transverse momentum distributions of charmonium states with the statistical hadronization modelarXiv:1901.09200.

[780] F.-K. Guo, Novel method for precisely measuring the X(3872) massarXiv:1902.11221.

[781] Q. Wu, G. Li, F. Shao, Q. Wang, R. Wang, Y. Zhang, Y. Zheng, Production of $X_{b}$ in $\Upsilon(5 S, 6 S) \rightarrow$ $\gamma X_{b}$ Radiative Decays, Adv. High Energy Phys. 2016 (2016) 3729050. arXiv:1606.05118, doi: $10.1155 / 2016 / 3729050$.

[782] P. González, A quark model study of strong decays of X (3915), J. Phys. G44 (7) (2017) 075004. arXiv:1611.03710, doi:10.1088/1361-6471/aa6d8a.

[783] X. Chen, X. Lü, R. Shi, X. Guo, Q. Wang, Radiative decay of hadronic molecule state for quarksarXiv: 1810.07347.

[784] X. Chen, X. Lü, Decay width of hadronic molecule structure for quarks, Phys. Rev. D97 (11) (2018) 114005. doi:10.1103/PhysRevD.97.114005.

[785] D.-Y. Chen, X. Liu, X.-Q. Li, H.-W. Ke, Unified Fano-like interference picture for charmoniumlike states Y(4008), Y(4260) and Y(4360), Phys. Rev. D93 (2016) 014011. arXiv:1512.04157, doi: 10.1103/PhysRevD.93.014011.

[786] D.-Y. Chen, X. Liu, T. Matsuki, Interference effect as resonance killer of newly observed charmoniumlike states $Y(4320)$ and $Y(4390)$, Eur. Phys. J. C78 (2) (2018) 136. arXiv:1708.01954, doi:10.1140/epjc/s10052-018-5635-1.

[787] J.-Z. Wang, D.-Y. Chen, X. Liu, T. Matsuki, Constructing $J / \psi$ family with updated data of charmoniumlike $Y$ statesarXiv:1903.07115. 
[788] X. Liu, H.-W. Ke, X. Liu, X.-Q. Li, Study of structures and dynamical decay mechanisms for multiquark systems, Phys. Rev. D93 (7) (2016) 074013. arXiv:1602.00226, doi:10.1103/PhysRevD. 93.074013.

[789] Y.-Y. Wang, Q.-F. Lü, E. Wang, D.-M. li, Role of $Y(4630)$ in the $p \bar{p} \rightarrow \Lambda_{c} \bar{\Lambda}_{c}$ reaction near threshold, Phys. Rev. D94 (2016) 014025. arXiv:1604.01553, doi:10.1103/PhysRevD.94.014025.

[790] J.-Z. Wang, H. Xu, J.-J. Xie, X. Liu, Production of the charmoniumlike state Y(4220) through the $p \bar{p} \rightarrow Y(4220) \pi^{0}$ reaction, Phys. Rev. D96 (9) (2017) 094004. arXiv:1710.08738, doi: 10.1103/PhysRevD.96.094004.

[791] M. Cleven, Q. Zhao, Cross section line shape of $e^{+} e^{-} \rightarrow \chi_{c 0} \omega$ around the $Y(4260)$ mass region, Phys. Lett. B768 (2017) 52-56. arXiv:1611.04408, doi:10.1016/j.physletb.2017.02.041.

[792] S.-R. Xue, H.-J. Jing, F.-K. Guo, Q. Zhao, Disentangling the role of the $Y(4260)$ in $e^{+} e^{-} \rightarrow$ $D^{*} \bar{D}^{*}$ and $D_{s}^{*} \bar{D}_{s}^{*}$ via line shape studies, Phys. Lett. B779 (2018) 402-408. arXiv:1708.06961, doi:10.1016/j.physletb.2018.02.027.

[793] L.-Y. Dai, J. Haidenbauer, U. G. Meissner, Re-examining the $X(4630)$ resonance in the reaction $e^{+} e^{-} \rightarrow \Lambda_{c}^{+} \bar{\Lambda}_{c}^{-}$, Phys. Rev. D96 (11) (2017) 116001. arXiv:1710.03142, doi:10.1103/PhysRevD. 96.116001 .

[794] J. Zhang, L. Yuan, R. Wang, Study on the resonant parameters of $Y(4220)$ and $Y(4390)$, Adv. High Energy Phys. 2018 (2018) 5428734. arXiv:1805.03565, doi:10.1155/2018/5428734.

[795] M. Piotrowska, F. Giacosa, P. Kovacs, Can the $\psi(4040)$ explain the peak associated with $Y(4008)$ ?, Eur. Phys. J. C79 (2) (2019) 98. arXiv:1810.03495, doi:10.1140/epjc/s10052-019-6615-9.

[796] S. Coito, F. Giacosa, On the origin of the Y(4260)arXiv: 1902.09268.

[797] Y.-H. Chen, L.-Y. Dai, F.-K. Guo, B. Kubis, On the nature of the $Y(4260)$ : a light-quark perspectivearXiv:1902.10957.

[798] Q. Wang, C. Hanhart, Q. Zhao, Decoding the riddle of $Y(4260)$ and $Z_{c}(3900)$, Phys. Rev. Lett. 111 (13) (2013) 132003. arXiv:1303.6355, doi:10.1103/PhysRevLett.111.132003.

[799] D.-Y. Chen, Y.-B. Dong, M.-T. Li, W.-L. Wang, Pionic transition from Y(4260) to Z $Z_{c}(3900)$ in a hadronic molecular scenario, Eur. Phys. J. A52 (10) (2016) 310. doi:10.1140/epja/ i2016-16310-0.

[800] D.-Y. Chen, J. He, C.-Q. Pang, Z.-Y. Zhou, Y(4320) and $Y(4390)$ as the candidate for $\psi\left(3^{3} D_{1}\right)$ charmoniumarXiv: 1804.00614.

[801] Q.-R. Gong, Z.-H. Guo, C. Meng, G.-Y. Tang, Y.-F. Wang, H.-Q. Zheng, $Z_{c}(3900)$ as a $D \bar{D}^{*}$ molecule from the pole counting rule, Phys. Rev. D94 (11) (2016) 114019. arXiv:1604.08836, doi:10.1103/PhysRevD.94.114019.

[802] D. Morgan, Pole counting and resonance classification, Nucl. Phys. A543 (1992) 632-644. doi: 10.1016/0375-9474(92)90550-4.

[803] K. L. Au, D. Morgan, M. R. Pennington, Meson Dynamics Beyond the Quark Model: A Study of Final State Interactions, Phys. Rev. D35 (1987) 1633. doi :10.1103/PhysRevD.35.1633. 
[804] Q.-R. Gong, J.-L. Pang, Y.-F. Wang, H.-Q. Zheng, The $Z_{c}(3900)$ peak does not come from the triangle singularity, Eur. Phys. J. C78 (4) (2018) 276. arXiv:1612.08159, doi:10.1140/epjc/ s10052-018-5690-7.

[805] M. Albaladejo, P. Fernandez-Soler, J. Nieves, $Z_{c}(3900)$ : Confronting theory and lattice simulations, Eur. Phys. J. C76 (10) (2016) 573. arXiv:1606.03008, doi:10.1140/epjc/ s10052-016-4427-8.

[806] A. Pilloni, C. Fernandez-Ramirez, A. Jackura, V. Mathieu, M. Mikhasenko, J. Nys, A. P. Szczepaniak, Amplitude analysis and the nature of the $\mathrm{Z}_{c}(3900)$, Phys. Lett. B772 (2017) 200-209. arXiv:1612.06490, doi:10.1016/j.physletb.2017.06.030.

[807] Z. Yang, Q. Wang, U.-G. Meissner, Isospin analysis of $B \rightarrow D^{*} \bar{D} K$ and the absence of the $Z_{c}(3900)$ in $B$ decays, Phys. Lett. B775 (2017) 50-53. arXiv:1706.00960, doi:10.1016/j. physletb.2017.10.049.

[808] J. He, D.-Y. Chen, $Z_{c}(3900) / Z_{c}(3885)$ as a virtual state from $\pi J / \psi-\bar{D}^{*} D$ interaction, Eur. Phys. J. C78 (2) (2018) 94. arXiv:1712.05653, doi:10.1140/epjc/s10052-018-5580-z.

[809] Q. Zhao, Some insights into the newly observed $Z_{c}(4100)$ in $B^{0} \rightarrow \eta_{c} K^{+} \pi^{-}$by LHCbarXiv: 1811.05357.

[810] X. Cao, J.-P. Dai, The spin parity of $Z_{c}^{-}(4100), Z_{1}^{+}(4050)$ and $Z_{2}^{+}(4250) \operatorname{arXiv:1811.06434.}$

[811] R. Mizuk, et al., Observation of two resonance-like structures in the $\pi^{+} \chi_{c 1}$ mass distribution in exclusive $\bar{B}^{0} \rightarrow K^{-} \pi^{+} \chi_{c 1}$ decays, Phys. Rev. D78 (2008) 072004. arXiv:0806.4098, doi: 10.1103/PhysRevD.78.072004.

[812] S. X. Nakamura, K. Tsushima, $Z_{c}(4430)$ and $Z_{c}(4200)$ as triangle singularitiesarXiv:1901.07385.

[813] S. X. Nakamura, Triangle singularities in $\bar{B}^{0} \rightarrow \chi_{c 1} K^{-} \pi^{+}$relevant to $Z_{1}(4050)$ and $Z_{2}(4250) \operatorname{arXiv:1903.08098.}$

[814] Q. Wu, D.-Y. Chen, X.-J. Fan, G. Li, Production of $Z_{c}(3900)$ and $Z_{c}(4020)$ in $B_{c}$ decayarXiv: 1902.05737.

[815] H.-W. Ke, X.-Q. Li, Study on decays of $Z_{c}(4020)$ and $Z_{c}(3900)$ into $h_{c}+\pi$, Eur. Phys. J. C76 (6) (2016) 334. arXiv:1601.03575, doi:10.1140/epjc/s10052-016-4183-9.

[816] D. A. S. Molnar, I. Danilkin, M. Vanderhaeghen, The role of charged exotic states in $e^{+} e^{-} \rightarrow$ $\psi(2 S) \pi^{+} \pi^{-}$arXiv: 1903.08458.

[817] S. S. Agaev, K. Azizi, H. Sundu, Strong $Z_{c}^{+}(3900) \rightarrow J / \psi \pi^{+} ; \eta_{c} \rho^{+}$decays in QCD, Phys. Rev. D93 (7) (2016) 074002. arXiv:1601.03847, doi:10.1103/PhysRevD.93.074002.

[818] Q. Wu, G. Li, F. Shao, R. Wang, Investigations on the charmless decay modes of Zc(3900) and Zc(4025), Phys. Rev. D94 (1) (2016) 014015. doi:10.1103/PhysRevD.94.014015.

[819] F. Goerke, T. Gutsche, M. A. Ivanov, J. G. Korner, V. E. Lyubovitskij, P. Santorelli, Fourquark structure of Zc(3900), Z(4430) and Xb(5568) states, Phys. Rev. D94 (9) (2016) 094017. arXiv:1608.04656, doi:10.1103/PhysRevD .94.094017.

[820] X.-H. Liu, L. Ma, L.-P. Sun, X. Liu, S.-L. Zhu, Resolving the puzzling decay patterns of charged $Z_{c}$ and $Z_{b}$ states, Phys. Rev. D90 (7) (2014) 074020. arXiv:1407.3684, doi:10.1103/PhysRevD. 90.074020 . 
[821] M. B. Voloshin, Radiative and pionic transitions $Z_{c}(4020)^{0} \rightarrow X(3872) \gamma$ and $Z_{c}(4020)^{ \pm} \rightarrow$ $X(3872) \pi^{ \pm}$arXiv: 1902.01281.

[822] Y.-H. Chen, J. T. Daub, F.-K. Guo, B. Kubis, U.-G. Meissner, B.-S. Zou, Effect of $Z_{b}$ states on $\Upsilon(3 S) \rightarrow \Upsilon(1 S) \pi \pi$ decays, Phys. Rev. D93 (3) (2016) 034030. arXiv:1512.03583, doi: 10.1103/PhysRevD.93.034030.

[823] Y.-H. Chen, M. Cleven, J. T. Daub, F.-K. Guo, C. Hanhart, B. Kubis, U.-G. Meissner, B.-S. Zou, Effects of $Z_{b}$ states and bottom meson loops on $\Upsilon(4 S) \rightarrow \Upsilon(1 S, 2 S) \pi^{+} \pi^{-}$transitions, Phys. Rev. D95 (3) (2017) 034022. arXiv:1611.00913, doi:10.1103/PhysRevD.95.034022.

[824] F. K. Guo, C. Hanhart, Yu. S. Kalashnikova, P. Matuschek, R. V. Mizuk, A. V. Nefediev, Q. Wang, J. L. Wynen, Interplay of quark and meson degrees of freedom in near-threshold states: A practical parametrization for line shapes, Phys. Rev. D93 (7) (2016) 074031. arXiv:1602.00940, doi: 10.1103/PhysRevD.93.074031.

[825] A. E. Bondar, M. B. Voloshin, $\Upsilon(6 S)$ and triangle singularity in $e^{+} e^{-} \rightarrow B_{1}(5721) \bar{B} \rightarrow$ $Z_{b}(10610) \pi$, Phys. Rev. D93 (9) (2016) 094008. arXiv:1603.08436, doi:10.1103/PhysRevD. 93.094008.

[826] C.-J. Xiao, D.-Y. Chen, Analysis of the hidden bottom decays of Zb(10610) and Zb(10650) via final state interaction, Phys. Rev. D96 (1) (2017) 014035. doi:10.1103/PhysRevD.96.014035.

[827] C.-J. Xiao, D.-Y. Chen, Y.-B. Dong, W. Zuo, T. Matsuki, Understanding the $\eta_{c} \rho$ decay mode of $Z_{c}^{(\prime)}$ via final state interactionsarXiv: 1811.04688.

[828] C.-Z. Yuan, The XYZ states revisited, Int. J. Mod. Phys. A33 (21) (2018) 1830018. arXiv: 1808.01570, doi:10.1142/S0217751X18300181.

[829] M. B. Voloshin, Mixing model for bottomoniumlike $Z_{b}$ resonances, Phys. Rev. D96 (9) (2017) 094024. arXiv:1707.00565, doi:10.1103/PhysRevD.96.094024.

[830] Q. Wang, V. Baru, A. A. Filin, C. Hanhart, A. V. Nefediev, J. L. Wynen, Line shapes of the $Z_{b}(10610)$ and $Z_{b}(10650)$ in the elastic and inelastic channels revisited, Phys. Rev. D98 (7) (2018) 074023. arXiv:1805.07453, doi:10.1103/PhysRevD.98.074023.

[831] V. Baru, E. Epelbaum, A. A. Filin, C. Hanhart, A. V. Nefediev, Q. Wang, Spin partners $W_{b J}$ from the line shapes of the $Z_{b}(10610)$ and $Z_{b}(10650)$ arXiv:1901.10319.

[832] Q. Wu, D.-Y. Chen, F.-K. Guo, Production of the $Z_{b}^{(\prime)}$ states from the $\Upsilon(5 S, 6 S)$ decays, Phys. Rev. D99 (3) (2019) 034022. arXiv:1810.09696, doi:10.1103/PhysRevD.99.034022.

[833] F. Goerke, T. Gutsche, M. A. Ivanov, J. G. Körner, V. E. Lyubovitskij, $Z_{b}(10610)$ and $Z_{b}^{\prime}(10650)$ decays in a covariant quark model, Phys. Rev. D96 (5) (2017) 054028. arXiv:1707.00539, doi: 10.1103/PhysRevD.96.054028.

[834] M. B. Voloshin, Radiative and $\rho$ transitions between a heavy quarkonium and isovector fourquark states, Phys. Rev. D98 (3) (2018) 034025. arXiv:1806.05651, doi:10.1103/PhysRevD. 98.034025.

[835] A. Del Fabbro, D. Janc, M. Rosina, D. Treleani, Production and detection of doubly charmed tetraquarks, Phys. Rev. D71 (2005) 014008. arXiv:hep-ph/0408258, doi:10.1103/PhysRevD. 71.014008 . 
[836] A. Esposito, M. Papinutto, A. Pilloni, A. D. Polosa, N. Tantalo, Doubly charmed tetraquarks in $B_{c}$ and $\Xi_{b c}$ decays, Phys. Rev. D88 (5) (2013) 054029. arXiv:1307.2873, doi:10.1103/PhysRevD. 88.054029.

[837] J. Hong, S. Cho, T. Song, S. H. Lee, Hadronic effects on the $c c \bar{q} \bar{q}$ tetraquark state in relativistic heavy ion collisions, Phys. Rev. C98 (1) (2018) 014913. arXiv:1804.05336, doi: 10.1103/PhysRevC.98.014913.

[838] A. Ali, A. Y. Parkhomenko, Q. Qin, W. Wang, Prospects of discovering stable double-heavy tetraquarks at a Tera- $Z$ factory, Phys. Lett. B782 (2018) 412-420. arXiv:1805.02535, doi: $10.1016 / j$.physletb.2018.05.055.

[839] A. Ali, Q. Qin, W. Wang, Discovery potential of stable and near-threshold doubly heavy tetraquarks at the LHC, Phys. Lett. B785 (2018) 605-609. arXiv:1806.09288, doi:10.1016/j. physletb.2018.09.018.

[840] T. Gershon, A. Poluektov, Displaced $\mathrm{B}_{c}^{-}$mesons as an inclusive signature of weakly decaying double beauty hadrons, JHEP 01 (2019) 019. arXiv:1810.06657, doi:10.1007/JHEP01(2019) 019.

[841] A. K. Ridgway, M. B. Wise, An Estimate of the Inclusive Branching Ratio to $\bar{B}_{c}$ in $\Xi_{b b q}$ DecayarXiv: 1902.04582.

[842] Y. Jin, S.-Y. Li, Z.-G. Si, Z.-J. Yang, T. Yao, Colour connections of four quark $Q \bar{Q} Q^{\prime} \bar{Q}^{\prime}$ system and doubly heavy baryon production in $e^{+} e^{-}$annihilation, Phys. Lett. B727 (2013) 468-473. arXiv:1309.5849, doi:10.1016/j.physletb.2013.10.070.

[843] E. Eichten, Z. Liu, Would a Deeply Bound $b \bar{b} b \bar{b}$ Tetraquark Meson be Observed at the LHC?arXiv: 1709.09605.

[844] R. Vega-Morales, R. Vega-Morales, Golden Probe of the di-؟ ThresholdarXiv: 1710.02738.

[845] G. Li, X.-F. Wang, Y. Xing, Fully Heavy Tetraquark $b b \bar{c} \bar{c}$ : Lifetimes and Weak DecaysarXiv: 1902.05805.

[846] L. Roca, J. Nieves, E. Oset, LHCb pentaquark as a $\bar{D}^{*} \Sigma_{c}-\bar{D}^{*} \Sigma_{c}^{*}$ molecular state, Phys. Rev. D92 (9) (2015) 094003. arXiv:1507.04249, doi:10.1103/PhysRevD.92.094003.

[847] L. Roca, E. Oset, On the hidden charm pentaquarks in $\Lambda_{b} \rightarrow J / \psi K^{-} p$ decay, Eur. Phys. J. C76 (11) (2016) 591. arXiv:1602.06791, doi:10.1140/epjc/s10052-016-4407-z.

[848] C. W. Xiao, $J / \psi N$ interactions revisited and $\Lambda_{b}^{0} \rightarrow J / \psi K^{-}\left(\pi^{-}\right) p$ decays, Phys. Rev. D95 (1) (2017) 014006. arXiv:1609.02712, doi:10.1103/PhysRevD.95.014006.

[849] J.-X. Lu, E. Wang, J.-J. Xie, L.-S. Geng, E. Oset, The $\Lambda_{b} \rightarrow J / \psi K^{0} \Lambda$ reaction and a hiddencharm pentaquark state with strangeness, Phys. Rev. D93 (2016) 094009. arXiv:1601.00075, doi:10.1103/PhysRevD.93.094009.

[850] Y. Huang, J.-J. Xie, J. He, X. Chen, H.-F. Zhang, Photoproduction of hidden-charm states in the $\gamma p \rightarrow \bar{D}^{* 0} \Lambda_{c}^{+}$reaction near threshold, Chin. Phys. C40 (12) (2016) 124104. arXiv:1604.05969, doi:10.1088/1674-1137/40/12/124104. 
[851] C. Cheng, X.-Y. Wang, The production of neutral $N^{*}(11052)$ resonance with hidden beauty from $\pi^{-} p$ scattering, Adv. High Energy Phys. 2017 (2017) 9398732. arXiv:1612.08822, doi: $10.1155 / 2017 / 9398732$.

[852] J.-J. Xie, W.-H. Liang, E. Oset, Hidden charm pentaquark and $\Lambda(1405)$ in the $\Lambda_{b}^{0} \rightarrow \eta_{c} K^{-} p(\pi \Sigma)$ reaction, Phys. Lett. B777 (2018) 447-452. arXiv:1711.01710, doi:10.1016/j.physletb. 2017. 12.064.

[853] R.-Q. Wang, J. Song, K.-J. Sun, L.-W. Chen, G. Li, F.-L. Shao, Hidden-charm pentaquark states in heavy ion collisions at energies available at the CERN Large Hadron Collider, Phys. Rev. C94 (4) (2016) 044913. arXiv:1601.02835, doi:10.1103/PhysRevC.94.044913.

[854] I. Schmidt, M. Siddikov, Production of pentaquarks in $p A$-collisions, Phys. Rev. D93 (9) (2016) 094005. arXiv:1601.05621, doi:10.1103/PhysRevD.93.094005.

[855] X.-H. Liu, M. Oka, Understanding the nature of heavy pentaquarks and searching for them in pion-induced reactions, Nucl. Phys. A954 (2016) 352-364. arXiv:1602.07069, doi:10.1016/j. nuclphysa.2016.04.040.

[856] F.-K. Guo, U. G. Meissner, J. Nieves, Z. Yang, Remarks on the $P_{c}$ structures and triangle singularities, Eur. Phys. J. A52 (10) (2016) 318. arXiv:1605.05113, doi:10.1140/epja/i2016-16318-4.

[857] M. Bayar, F. Aceti, F.-K. Guo, E. Oset, A Discussion on Triangle Singularities in the $\Lambda_{b} \rightarrow$ $J / \psi K^{-} p$ Reaction, Phys. Rev. D94 (7) (2016) 074039. arXiv:1609.04133, doi:10.1103/ PhysRevD.94.074039.

[858] S.-H. Kim, H.-C. Kim, A. Hosaka, Heavy pentaquark states $P_{c}(4380)$ and $P_{c}(4450)$ in the $J / \psi$ production induced by pion beams off the nucleon, Phys. Lett. B763 (2016) 358-364. arXiv: 1605.02919, doi:10.1016/j.physletb.2016.10.061.

[859] M. Karliner, J. L. Rosner, $J / \psi N$ photoproduction on deuterium as a test for exotic baryonsarXiv: 1705.07691.

[860] P. Zhou, Y. K. Hsiao, C. Q. Geng, Pentaquarks in semileptonic $\Lambda_{b}$ decays, Annals Phys. 383 (2017) 278-284. arXiv:1706.02460, doi:10.1016/j.aop.2017.06.001.

[861] A. Pilloni, J. Nys, M. Mikhasenko, M. Albaladejo, C. Fernndez-Ramírez, A. Jackura, V. Mathieu, N. Sherrill, T. Skwarnicki, A. P. Szczepaniak, What is the right formalism to search for resonances? II. The pentaquark chain, Eur. Phys. J. C78 (9) (2018) 727. arXiv:1805.02113, doi:10.1140/ epjc/s10052-018-6177-2.

[862] E. Ya. Paryev, Yu. T. Kiselev, The role of hidden-charm pentaquark resonance $P_{c}^{+}(4450)$, in $J / \psi$ photoproduction on nuclei near threshold, Nucl. Phys. A978 (2018) 201-213. arXiv:1810.01715, doi:10.1016/j.nuclphysa.2018.08.009.

[863] M. B. Voloshin, Hidden-charm pentaquark formation in antiproton - deuterium collisionsarXiv: 1903.04422.

[864] Q.-F. Lu, Y.-B. Dong, Strong decay mode $J / \psi p$ of hidden charm pentaquark states $P_{c}^{+}(4380)$ and $P_{c}^{+}(4450)$ in $\Sigma_{c} \bar{D}^{*}$ molecular scenario, Phys. Rev. D93 (7) (2016) 074020. arXiv:1603.00559, doi:10.1103/PhysRevD.93.074020. 
[865] C.-W. Shen, F.-K. Guo, J.-J. Xie, B.-S. Zou, Disentangling the hadronic molecule nature of the $P_{c}(4380)$ pentaquark-like structure, Nucl. Phys. A954 (2016) 393-405. arXiv:1603.04672, doi:10.1016/j.nuclphysa.2016.04.034.

[866] Y.-H. Lin, C.-W. Shen, F.-K. Guo, B.-S. Zou, Decay behaviors of the $P_{c}$ hadronic molecules, Phys. Rev. D95 (11) (2017) 114017. arXiv:1703.01045, doi:10.1103/PhysRevD.95.114017.

[867] Y.-H. Lin, C.-W. Shen, B.-S. Zou, Decay behavior of the strange and beauty partners of $P_{c}$ hadronic molecules, Nucl. Phys. A980 (2018) 21-31. arXiv:1805.06843, doi:10.1016/j. nuclphysa.2018.10.001.

[868] M. I. Eides, V. Yu. Petrov, Decays of pentaquarks in hadrocharmonium and molecular scenarios, Phys. Rev. D98 (11) (2018) 114037. arXiv:1811.01691, doi:10.1103/PhysRevD.98.114037.

[869] Y. Jin, S.-Y. Li, S.-Q. Li, New $B_{s}^{0} \pi^{ \pm}$and $D_{s}^{ \pm} \pi^{ \pm}$states in high energy multiproduction process, Phys. Rev. D94 (1) (2016) 014023. arXiv:1603.03250, doi:10.1103/PhysRevD.94.014023.

[870] A. Cerri, et al., Opportunities in Flavour Physics at the HL-LHC and HE-LHCarXiv : 1812.07638.

[871] M. Ablikim, et al., Observation of $e^{+} e^{-} \rightarrow \pi^{+} \pi^{-} \psi(3770)$ and $D_{1}(2420)^{0} \bar{D}^{0}+$ c.c.arXiv:1903. 08126.

[872] M. Ablikim, et al., Cross section measurements of $e^{+} e^{-} \rightarrow \omega \chi_{c 0}$ from $\sqrt{s}=4.178$ to 4.278 GeVarXiv:1903.02359.

[873] R. Aaij, et al., Observation of $C P$ violation in charm decaysarXiv: 1903.08726.

[874] W. Altmannshofer, et al., The Belle II Physics BookarXiv: 1808.10567. 